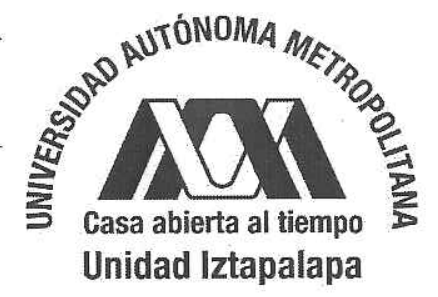

\title{
MORFISMOS ENTRE RETÍCULAS DE PRERRADICALES ASOCIADOS A FUNTORES ENTRE \\ CATEGORÍAS DE MÓDULOS
}

Tesis que presenta

M. en C. JANETH ANABELLE MAGAÑA ZAPATA

para obtener el grado de Doctor en Ciencias (Matemáticas)

Asesor

Dr. ROGELIO FERNÁNDEZ-ALONSO GONZÁLEZ

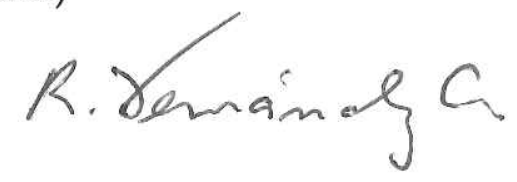

Jurado Evaluador:

Presidente: Dr. José Ríos Montes JoséRios M.

Secretario:

Dra. María José Arroyo Paniagua

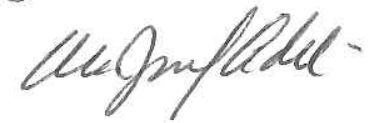

Vocales:

Dr. Carlos José Enrique Signoret Poillon

Dr. Francisco Marmolejo Rivas

Ciudad de México a 9 de junio de 2016 


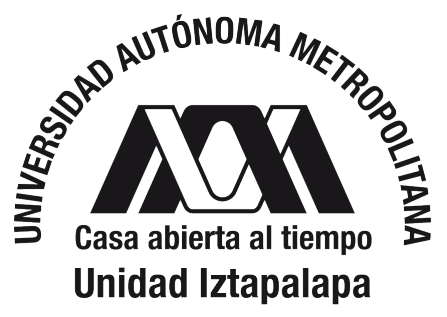

\title{
MORFISMOS ENTRE RETÍCULAS DE PRERRADICALES ASOCIADOS A FUNTORES ENTRE CATEGORÍAS DE MÓDULOS
}

\author{
Tesis que presenta \\ M. en C. JANETH ANABELLE MAGAÑA ZAPATA \\ para obtener el grado de Doctor en Ciencias (Matemáticas) \\ Asesor \\ Dr. ROGELIO FERNÁNDEZ-ALONSO GONZÁLEZ
}

Jurado Evaluador:

Presidente: Dr. José Ríos Montes

Secretario: $\quad$ Dra. María José Arroyo Paniagua

Vocales:

Dr. Carlos José Enrique Signoret Poillon

Dr. Francisco Marmolejo Rivas

Ciudad de México a 9 de junio de 2016 


\title{
UNIVERSIDAD AUTÓNOMA METROPOLITANA \\ Iztapalapa
}

\section{MORFISMOS ENTRE RETÍCULAS DE PRERRADICALES ASOCIADOS A FUNTORES ENTRE CATEGORÍAS DE MÓDULOS}

\author{
Presenta \\ M. en C. JANETH ANABELLE MAGAÑA ZAPATA \\ Asesor \\ Dr. ROGELIO FERNÁNDEZ-ALONSO GONZÁLEZ
}

TESIS

PARA OBTENER EL GRADO DE DOCTOR EN CIENCIAS MATEMÁTICAS 


\section{Agradecimientos}

Agradezco al Dr. Rogelio Fernández-Alonso González por haberme permitido realizar este trabajo de investigación bajo su dirección. Le agradezco sus valiosas ideas que compartió conmigo para la elaboración de este trabajo, así como las interesantes matemáticas que me ha enseñado. De manera especial le agradezco toda la paciencia que tuvo conmigo, su amistad y el tiempo que me brindó durante mis estudios de doctorado.

Sin su invaluable conocimento esta tesis no hubiera sido concluida. También, muchas gracias por ampliar mi formación académica.

A los sinodales, Dra. María José Arroyo, Dr. Francisco Marmolejo, Dr. José Ríos y Dr. Carlos Signoret, por haber acepado formar parte del comité evaluador para este trabajo. Les gradezco todo el tiempo invertido para mejorar este manuscrito, así como sus excelentes y atinadas observaciones y sugerencias.

Dra. María José, gracias por los ánimos que me dió durante la etapa de revisión.

Dr. Francisco Marmolejo, muchas gracias por las interesantes ideas que compartió conmigo para enriquecer esta tesis.

A la UAM-I, por las facilidades otorgadas durante mis estudios de maestría y doctorado y sobre todo por la excelente formación académica y humana que me ha brindado. En especial agradezco al Dr. Horacio Tapia Recillas, puesto que si no hubiera sido por sus seminarios en la Facultad de Matemáticas de la UADY, yo no hubiera conocido esta MARAVILLOSA UNIVERSIDAD.

A la M. en C. Iseo González, por toda su ayuda en los procesos administrativos, así como por el trato amable y de confianza que me ha mostrado durante toda mi estancia en la UAM-I.

Al Consejo Nacional de Ciencia y Tecnología ( CONACYT ), por los apoyos económicos que me otorgó durante mis estudios de maestría y doctorado.

A la Fundación Sofía Kovalévskaia-Sociedad Matemática Mexicana, por el reconocimiento otorgado referente a este trabajo de investigación y por promover la participación de las mujeres en la investigación en Matemáticas. 
A mis padres:

María Herminia y Juan Magaña 



\section{Índice general}

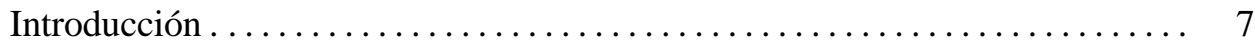

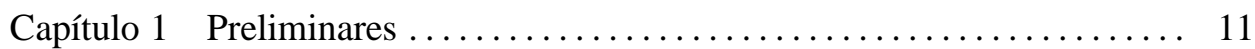

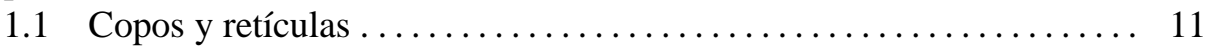

1.2 Conexiones de Galois ................................... 13

1.3 Funtores y transformaciones naturales ................... 15

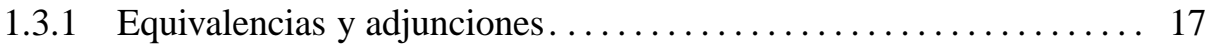

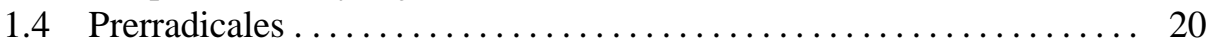

1.4.1 Prerradicales idempotentes y radicales $\ldots \ldots \ldots \ldots \ldots \ldots \ldots \ldots 23$

1.4.2 Particiones de $R$-pr inducida por una clase de módulos ....... 28

Capítulo 2 Conexiones entre retículas de prerradicales.............. 29

2.1 Las retículas $R$-pr y $S$-pr cuando $R$ y $S$ son Morita-equivalentes . . . 29

2.2 Las retículas $R$-pr y $S$-pr cuando hay una situación de adjunción entre

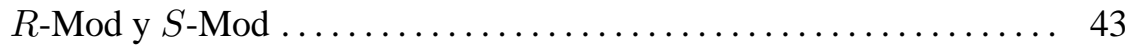

2.3 Propiedades generales de la conexión de Galois

$\langle R$-pr, $\varphi, \psi, S$-pr $\rangle$ inducida por la adjunción $\langle F, G\rangle \ldots \ldots \ldots \ldots \ldots 47$

2.4 Conexión de Galois inducida por el funtor tensor y el funtor Hom . . 54

Capítulo 3 Morfismos entre retículas de prerradicales asociados a transformaciones naturales ........................ 63

$3.1 \varphi_{\gamma}$ y $\psi_{\gamma}$, con $\gamma$ una transformación natural general ............66 63

$3.2 \varphi_{\gamma}$ y $\psi_{\gamma}$ para transformaciones naturales $\gamma$ específicas............ 70

3.3 Morfismos asociados a funtores ..................... 82

3.4 Morfismos asociados a prerradicales y coprerradicales . ......... 90

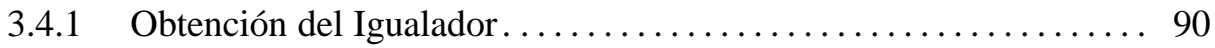

3.4.2 Obtención del Coigualador ......................... 92

3.4 .3 Obtención del Anulador ............................... 94

3.4.4 Obtención del Totalizador ......................... 95

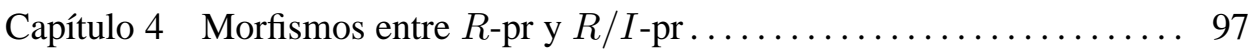

4.1 Conexión de Galois entre las retículas $R$-pr y $R / I$-pr......... 97

4.2 Particiones de $R$-pr inducidas por un ideal $I$ de $R \ldots \ldots \ldots \ldots \ldots 103$

Capítulo 5 Aplicaciones a grupos abelianos .................... 111

5.1 Particiones de $\mathbb{Z}$-pr inducidas por el ideal $p^{n} \mathbb{Z} \ldots \ldots \ldots \ldots \ldots \ldots 111$

5.1.1 Intervalos en $\mathbb{Z}$-pr inducidos por prerradicales idempotentes y

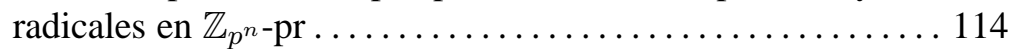


5.1.2 Prerradicales idempotentes y radicales en $\mathbb{Z}$-pr.............. 122

5.1 .3 Radicales idempotentes en $\mathbb{Z}$-pr ...................... 128

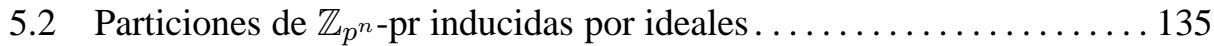

5.3 Particiones de $\mathbb{Z}_{(p)}$-pr inducidas por ideales $\ldots \ldots \ldots \ldots \ldots \ldots \ldots . \ldots \ldots$

Conclusiones y Perspectivas ............................. 139

Bibliografía........................................ 141

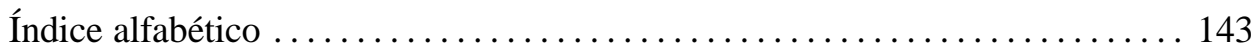




\section{Introducción}

Dado un anillo $R$ asociativo con 1 , se denota como $R$-pr a la clase de todos los prerradicales sobre el anillo $R$. Se conoce que $R$-pr es una gran retícula completa que no necesariamente es un conjunto, más aún, es una gran retícula atómica y coatómica. Con base en las operaciones del producto y el coproducto en $R$-pr, se conoce la caracterización de algunas clases de prerradicales, tales como la clase de los idempotentes y la clase de los radicales.

Una manera de obtener propiedades de ciertos anillos y módulos es a partir del estudio de los prerradicales y viceversa. Por ejemplo se sabe que si $R$ es un anillo tal que $R$-pr es una retícula booleana, entonces $R$ debe ser un anillo semisimple. Recíprocamente si $R$ es un anillo semisimple tal que $\mid R$-Simp $\mid=n$, donde $R$-Simp es un conjunto completo e irredundante de representantes de las clases de isomorfismos de $R$-módulos simples, entonces $R$-pr es una retícula booleana finita de cardinalidad $2^{n}$.

En este trabajo se aborda de otra manera esta relación entre anillos, módulos y prerradicales, que consiste a grandes rasgos en: dados dos anillos $R$ y $S$ relacionados o conectados de alguna manera, ¿cuál es la relación entre sus retículas de prerradicales?

En la Proposición 5.2 de [3] y la Proposición I.9.2 de [4], Bican, Kepka, Nẽmec y Jambor demuestran que cuando los anillos $R$ y $S$ son Morita-equivalentes las retículas $R$-pr y $S$-pr son isomorfas. En el presente trabajo extendemos dicho resultado para un caso más general en el que se sustituye la condición de que exista una equivalencia entre las categorías $R$-Mod y $S$-Mod, por la condición de que exista una situación de adjunción entre ambas categorías, probando en este caso que hay una conexión de Galois entre las retículas correspondientes de prerradicales. Más aún definimos morfismos entre retículas de prerradicales sin tener una situación de adjunción entre las categorías de módulos respectivas.

Un ejemplo de lo que mencionamos en el párrafo anterior y que abordamos en este texto es la conexión de Galois entre las retículas $R$-pr y $R / I$-pr inducida por la situación de adjunción entre las categorías $R$-Mod y $R / I$-Mod, donde $I$ es un ideal de $R$. Gran parte de este trabajo está centrado en esta conexión de Galois y de la cual también obtenemos otros ejemplos.

Organizamos este trabajo en cinco capítulos y un último apartado que contiene algunas posibles líneas de investigación. A continuación presentamos una breve descripción del contenido de cada uno de los cinco capítulos de los que consta esta tesis. 
En el primer capítulo se incluyen algunos conceptos de la Teoría de Retículas y Módulos que usamos a lo largo de este trabajo, así como los resultados principales de la Teoría de Prerradicales y las conexiones de Galois que necesitamos para este escrito.

Tomando como base la demostración que se da en [3] y [4], en el segundo capítulo detallamos la prueba del isomorfismo que existe entre las retículas $R$-pr y $S$-pr cuando hay una equivalencia entre las categorías de módulos respectivas. No está de más decir, que la prueba que se presenta aquí es un tanto diferente a la que dan Bican, Kepka, Nẽmec y Jambor y con una notación distinta. Partiendo de este resultado, probamos el teorema principal de este capítulo, el cual establece la conexión de Galois $\langle R$-pr, $\varphi, \psi, S$-pr $\rangle$ inducida por la adjunción $\langle F, G\rangle$, donde $F: R$-Mod $\rightarrow S$-Mod y $G: S$-Mod $\rightarrow R$-Mod. También se muestran propiedades generales de tal conexión de Galois. Para finalizar el segundo capítulo estudiamos la conexión de Galois entre las retículas $R$-pr y $S$-pr inducida por la adjunción entre los funtores $F=U \bigotimes_{R}$ - y $G=\operatorname{Hom}_{S}\left(U,{ }_{-}\right)$, donde $U={ }_{S} U_{R}$ es un bimódulo. Este caso resulta ser muy general puesto que el Teorema de Watts-Eilenberg, ver [5] y [20], caracteriza a los funtores adjuntos cuya forma es precisamente la que usamos en este capítulo y por tanto la definición de $\operatorname{los}$ morfismos $\varphi$ y $\psi$ que describen a la conexión de Galois entre dichas retículas siempre tienen una forma específica, a saber, a cada $\tau \in R$-pr se le asigna la $\tau$-traza y a cada $\sigma \in S$-pr se le asigna el $\sigma$-anulador respecto al bimódulo $U$ respectivamente. Además, vemos que existen otras formas de establecer estas asignaciones $\varphi$ y $\psi$.

En el capítulo siguiente definimos morfismos entre las retículas de prerradicales $R$-pr y $S$-pr aún no teniendo una situación de adjunción entre las categorías de módulos respectivas y que extienden bien los resultados obtenidos en el segundo capítulo. En la primera parte del tercer capítulo definimos morfismos entre las retículas de prerradicales asociados a una transformación natural para luego dar algunos ejemplos de estos morfismos para transformaciones naturales específicas que involucran a los prerradicales, para algunos resultados duales damos la definición de coprerradical sobre la categoría $R$-Mod asociado a un prerradical. Al final de este capítulo trabajamos con los morfismos entre las retículas de prerradicales asociados a la transformación natural identidad sobre un funtor $F$ y aún más particular cuando el funtor es un prerradical o un coprerradical. En estos últimos casos obtenemos otras formas de cómo describir a los operadores que aparecen en la Definición 3.1 de [9], como imágenes de ciertos prerradicales bajo los morfismos asociados a prerradicales o coprerradicales.

En el cuarto capítulo como ejemplo de los resultados obtenidos en el capítulo 2 , se estudia la conexión de Galois $\left\langle\varphi_{I}, \psi_{I}\right\rangle$ entre las retículas $R$-pr y $R / I$-pr donde $I$ es un ideal de $R$. En este caso el morfismo $\varphi_{I}$ es suprayectivo y $\psi_{I}$ es inyectivo. En esta conexión de Galois obtenemos la descripción de los intervalos inducidos por la imagen inversa de un $\sigma \in R / I$-prerradical bajo el morfismo $\varphi_{I}$ y veremos varias descripciones de éstos. Además el extremo inferior de estos intervalos es el resultado de evaluar un morfismo que es parte de otra conexión de Galois, de la cual hablaremos al final de este capítulo. Con tales intervalos se obtienen particiones de 
la retícula $R$-pr que dependen del ideal $I$ y que estudiaremos para otros ejemplos específicos en el siguiente capítulo.

En el último capítulo presentamos varios ejemplos de la conexión de Galois entre las retículas $R$-pr y $R / I$-pr donde $I$ es un ideal de $R$. El primer ejemplo es cuando $R=\mathbb{Z}$ y considerando el ideal $I=p^{n} \mathbb{Z}$, donde $p$ es un primo y $n \geq 1$. Para la conexión de Galois entre $\mathbb{Z}$-pr y $\mathbb{Z}_{p^{n}}$-pr, los intervalos que inducen la ima-

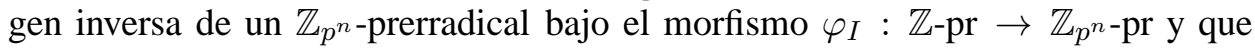
dependen del ideal $I$ se pueden encontrar fácilmente, puesto que la estructura de

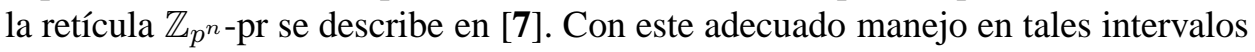
obtenemos varios resultados destacados en este capítulo como es la descripción de

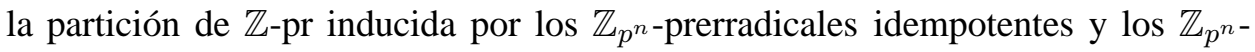
radicales, ya que las clases de estos tipos de $\mathbb{Z}_{p^{n}}$-prerradicales se caracterizan en [7]. Los intervalos en $\mathbb{Z}$-pr donde se encuentran los radicales idempotentes se describen en una manera muy sencilla y por tanto se caracterizan a todos los radicales exactos izquierdos sobre $\mathbb{Z}$, aunque la clase de los radicales idempotentes no se sabe si es un conjunto o no. Por último mostramos los ejemplos en donde $R=\mathbb{Z}_{p^{n}}$ y consideramos el ideal $I=p^{i} \mathbb{Z}_{p^{n}}$ con $i=1, \ldots, n-1$ y $p$ primo y el ejemplo en donde el anillo $R$ es la localización del anillo $\mathbb{Z}$ en el primo $p$. En todos los ejemplos de esta sección es importante la estructura de la retícula $\mathbb{Z}_{p^{n} \text {-pr. }}$ 



\section{Capítulo 1}

\section{Preliminares}

En este capítulo se presenta un pequeño resumen de la teoría que usaremos en este trabajo, así como la notación y terminología de la cuál haremos uso.

\subsection{Copos y retículas}

Primero recordemos los siguientes conceptos y resultados, los cuales se pueden extender a clases que no necesariamente son conjuntos. Lo referente a esta sección se puede consultar en [13], [17] y [19].

Definición 1.1. Un orden parcial sobre un conjunto no vacio $P$ es una relación binaria " $\leq$ " en $P$, la cual para todo $x, y, z \in P$ es:

1. Reflexiva: $x \leq x$.

2. Antisimétrica: Si $x \leq y$ y $y \leq x$, entonces $x=y$.

3. Transitiva: Si $x \leq y$ y $y \leq z$, entonces $x \leq z$.

Definición 1.2. Un conjunto parcialmente ordenado (copo) es una pareja $\langle P, \leq\rangle$, donde $P$ es un conjunto no vacío y " $\leq$ " es un orden parcial en $P$.

Observación 1.3. La expresión $y \geq x$ significa $x \leq y$. Si $x \leq y(y \geq x)$ y $x \neq y$, usaremos la expresión $x<y(y>x)$.

En algunas ocasiones cuando el orden parcial sea claro en $P$, usaremos $P$ para referirnos al copo $\langle P, \leq\rangle$.

Definición 1.4. Sea $\langle P, \leq\rangle$ un conjunto parcialmente ordenado y $Q \subseteq P$. Decimos que $p \in Q$ es:

1. un elemento menor de $Q$, si $p \leq x$ para todo $x \in Q$.

2. un elemento mínimo de $Q$, si no existe $x \in Q$ tal que $x<p$.

3. un elemento mayor de $Q$, si $x \leq p$ para todo $x \in Q$.

4. un elemento máximo de $Q$, si no existe $x \in Q$ tal que $p<x$.

Observación 1.5. Si un copo P tiene elemento menor o elemento mayor, éstos son únicos y se denotan respectivamente como $0_{P}$ y $1_{P}$.

Definición 1.6. Sea $\langle P, \leq\rangle$ un copo y $Q \subseteq P$. Decimos que $p \in P$ es:

1. una cota inferior de $Q$, si $p \leq q$ para todo $q \in Q$. Si existe el elemento mayor del conjunto de todas la cotas inferiores de $Q$, lo llamaremos el infimo de $Q$ y lo denotaremos como $\bigwedge Q$. 
2. una cota superior de $Q$, si $q \leq p$ para todo $q \in Q$. Si existe el elemento menor del conjunto de todas la cotas superiores de $Q$, lo llamaremos el supremo de $Q$ y lo denotaremos como $\bigvee Q$.

Definición 1.7. Sean $\langle P, \leq\rangle$ y $\left\langle P^{\prime}, \preceq\right\rangle$ copos. Una función $f: P \rightarrow P^{\prime}$,

1. es un morfismo de copos si preserva el orden. Es decir, si $p \leq p^{\prime}$, entonces $f(p) \preceq f\left(p^{\prime}\right)$ para todo $p, p^{\prime} \in P$.

2. es un isomorfismo de copos si es una función biyectiva tal que para todo $p, p^{\prime} \in P, p \leq p^{\prime}$ si y solo si $f(p) \preceq f\left(p^{\prime}\right)$.

En este trabajo estaremos trabajando con estructuras como la de la siguiente definición.

Definición 1.8. Una retícula (completa) es un copo P en el cual cada par de elementos (subconjunto) tiene supremo e infimo. Una retícula se denota usualmente como $\langle P, \leq, \vee, \wedge\rangle$.

Definición 1.9. Sean $\langle P, \leq, \vee, \wedge\rangle$ y $\left\langle P^{\prime}, \preceq, \vee, \wedge\right\rangle$ retículas. Una función $f: P \rightarrow$ $P^{\prime}$,

1. es un morfismo de retículas si $f(a \vee b)=f(a) \vee f(b)$ y $f(a \wedge b)=$ $f(a) \wedge f(b)$ para todo $a, b \in P$.

2. es un isomorfismo de retículas si es un morfismo de retículas biyectivo.

En el siguiente capítulo detallaremos un resultado el cual motivó este trabajo. La siguiente proposición la usaremos en la prueba de tal resultado.

Proposición 1.10. ( [19], Proposición 1.1 ) Si $P$ y $P^{\prime}$ son retículas completas y $f: P \rightarrow P^{\prime}$ es un isomorfismo de copos, entonces $f$ es un isomorfismo de retículas completas.

La siguiente sección la dedicaremos a las conexiones de Galois, por lo que los siguientes resultados están relacionados con dicho tema.

Definición 1.11. ( [6], Definición 2) Una función $f: P \rightarrow P$, donde $\langle P, \leq\rangle$ es un copo se llama operador cerradura (interior) si cumple lo siguiente:

1. f es un morfismo de copos,

2. $f$ es idempotente, es decir, $f(f(p))=f(p)$ para toda $p \in P y$

3. $f$ es inflatoria (deflatoria), es decir, $p \leq f(p)(f(p) \leq p)$ para toda $p \in P$.

Definición 1.12. ( [6], Definición 2 ) Un subconjunto $Q$ de un copo $\langle P, \leq\rangle$ es un sistema de cerrados (abiertos) de $\langle P, \leq\rangle$ si para cada $p \in P$ existe el menor (mayor) elemento $q \in Q$ tal que $p \leq q(p \geq q)$. Llamaremos a q la cerradura (el interior) de $p$. 
Obsérvese que dado un sistema de cerrados (abiertos), éste induce naturalmente un operador cerradura (interior) e inversamente dado un operador cerradura (interior) su imagen es un sistema de cerrados (abiertos).

Definición 1.13. Sea $f: P \rightarrow P$ un operador cerradura (interior). Llamaremos elementos cerrados (abiertos) de $P$ a los elementos de Im $f$.

Notemos que dado $f: P \rightarrow P$ un operador cerradura (interior), los elementos cerrados (abiertos) de $P$ son los puntos fijos del operador cerradura, es decir, los elementos $p \in P$ tal que $f(p)=p$.

El siguiente resultado es una caracterización de los sistemas de cerrados cuando se tiene una retícula completa.

Proposición 1.14. ( [6], Proposición 1 ) Sean $\langle P, \leq, \vee, \wedge\rangle$ una retícula completa y $Q \subseteq P$. Entonces son equivalentes:

a) $Q$ es un sistema de cerrados (abiertos) de $P$.

b) $Q$ es cerrado bajo ínfimos (supremos) arbitrarios.

\subsection{Conexiones de Galois}

En esta sección presentamos la teoría necesaria para este trabajo en cuanto a las conexiones de Galois entre conjuntos parcialmente ordenados (copos). A grandes rasgos una conexión de Galois conecta dos copos de una manera más débil que un isomorfismo. Para tener una idea de este concepto, recordemos el Teorema fundamental de la Teoría de Galois, el cual establece una correspondencia biunívoca entre las extensiones intermedias y los subgrupos del grupo de Galois de una extensión de campos que es Galois, dicho Teorema es un ejemplo muy especial de las conexiones de Galois. Lo referente a esta sección se puede consultar en [6].

Es importante señalar que los siguientes resultados se pueden extender a preórdenes y clases que no necesariamente son conjuntos.

Definición 1.15. ( [6], Definición 1 ) Sean $\langle P, \leq\rangle$ y $\langle Q, \preceq\rangle$ copos. Una conexión de Galois entre los copos $P$ y $Q$ consiste de dos funciones $f: P \rightarrow Q$ y $g: Q \rightarrow P$ tales que para toda $p \in P$ y $q \in Q$ se cumple que:

$$
p \leq g(q) \Leftrightarrow f(p) \preceq q .
$$

Se denota como $\langle P, f, g, Q\rangle$ a la conexión de Galois ${ }^{1}$ entre los copos $P$ y $Q$.

A las funciones $f$ y $g$ se les llama la parte coadjunta y adjunta de $\langle P, f, g, Q\rangle$ respectivamente.

En adelante cuando hablemos de conexiones de Galois nos estaremos refiriendo a una como la de la Definición 1.15.

La siguiente proposición contiene algunas propiedades de una conexión de Galois:

\footnotetext{
${ }^{1}$ Si $f$ y $g$ son funciones tales que para toda $p \in P$ y $q \in Q$ se cumple que $p \leq g(q) \Leftrightarrow q \preceq$ $f(p)$, entonces diremos que $\langle P, f, g, Q\rangle$ es una conexión de Galois antítona [18].
} 
Proposición 1.16. ( [6], Proposición 2 ) Sean $\langle P, \leq\rangle$ y $\langle Q, \preceq\rangle$ copos y $\langle P, f, g, Q\rangle$ una conexión de Galois. Entonces

a) Las funciones $f$ y g preservan el orden.

b) Las funciones $f$ y gon casi-inversas, es decir, $f \circ g \circ f=f$ y $g \circ f \circ g=g$.

c) La función $f$ preserva supremos y la función g preserva ínfimos.

d) Sea $p \in P$. Entonces $(g \circ f)(p)=p$ si y sólo si $p \in g(Q)$.

e) Sea $q \in Q$. Entonces $(f \circ g)(q)=q$ si y sólo si $q \in f(P)$.

En el siguiente capítulo probamos uno de los resultados principales de este trabajo usando una definición equivalente de conexión de Galois entre dos copos.

Proposición 1.17. ([6], Proposición 4) Sean $\langle P, \leq\rangle$ y $\langle Q, \preceq\rangle$ copos y $f: P \rightarrow Q$, $g: Q \rightarrow P$ funciones. Entonces las siguientes condiciones son equivalentes:

a) $\langle P, f, g, Q\rangle$ es una conexión de Galois.

b) Las funciones $f$ y $g$ preservan el orden, $g \circ f$ es inflatoria $y f \circ g$ es deflatoria.

Como consecuencia de la la Proposición 1.16 b), d), e) y la Proposición 1.17, se tiene el siguiente resultado.

Corolario 1.18. Sean $\langle P, \leq\rangle$ y $\langle Q, \preceq\rangle$ copos y $\langle P, f, g, Q\rangle$ una conexión de Galois. Entonces $g \circ f$ es un operador cerradura en $P$ y $f \circ g$ es un operador interior en $Q$. Además $(g \circ f)(P)=g(Q)$ y $(f \circ g)(Q)=f(P)$.

El Corolario 1.18 nos dice que los elementos cerrados de $P$ son los elementos $p \in g(Q)$. Dualmente los elementos abiertos de $Q$ son los elementos $q \in f(P)$.

Una consecuencia inmediata de las conexiones de Galois, con respecto a los elementos menor y mayor de las retículas en cuestión, es la que sigue.

Lema 1.19. Sean $\langle P, \leq\rangle$ y $\langle Q, \preceq\rangle$ retículas completas $y\langle P, f, g, Q\rangle$ una conexión de Galois. Entonces

a) $f\left(0_{P}\right)=0_{Q}$, es decir, $0_{Q}$ es un abierto de $Q$.

b) $g\left(1_{Q}\right)=1_{P}$, es decir, $1_{P}$ es un cerrado de $P$.

Demostración. Por ser $\langle P, f, g, Q\rangle$ una conexión de Galois, se tiene que $f \circ g$ es deflatoria, por lo que $(f \circ g)\left(0_{Q}\right) \preceq 0_{Q}$ y entonces $(f \circ g)\left(0_{Q}\right)=0_{Q}$. También $g\left(0_{Q}\right) \geq 0_{P}$, por lo que

$$
0_{Q}=(f \circ g)\left(0_{Q}\right) \succeq f\left(0_{P}\right) \succeq 0_{Q} .
$$

Por lo tanto $f\left(0_{P}\right)=0_{Q}$. Dualmente se prueba que $g\left(1_{Q}\right)=1_{P}{ }^{2}$.

Proposición 1.20. ( [6], Proposición 3 ) Sea $\langle P, f, g, Q\rangle$ una conexión de Galois. Entonces hay un isomorfismo de copos entre $g(Q)$ y $f(P)$. Es decir, hay un isomorfismo de copos entre los cerrados de $P$ y los abiertos de $Q$.

\footnotetext{
${ }^{2}$ La demostración también se sigue de las siguientes igualdades $f\left(0_{P}\right)=f(\vee \emptyset)=\vee \emptyset=0_{Q} \mathrm{y}$ $g\left(1_{Q}\right)=g(\wedge \emptyset)=\wedge \emptyset=1_{P}$
} 
Demostración. Sean $\bar{f}: g(Q) \rightarrow f(P)$ y $\bar{g}: f(P) \rightarrow g(Q)$ las funciones restricción-correstricción de $f$ y $g$ respectivamente. Entonces $\bar{f}$ y $\bar{g}$ preservan orden, puesto que las funciones $f$ y $g$ preservan orden. Así las funciones $\bar{f}$ y $\bar{g}$ son morfismos de copos. Ahora bien sea $p \in g(Q)$, entonces por el Corolario 1.18, $(g \circ f)(p)=p$. Luego, $(\bar{g} \circ \bar{f})(p)=\bar{g}(f(p))=g(f(p))=p$, de donde $\bar{g} \circ \bar{f}=1_{g(Q)}$. Análogamente $\bar{f} \circ \bar{g}=1_{f(P)}$. Por lo tanto concluimos que $g(Q) \mathrm{y}$ $f(P)$ son copos isomorfos.

En lo que sigue, usaremos la siguiente notación.

Observación 1.21. Cuando la conexión de Galois entre los copos $P$ y $Q$ sea clara, usaremos $\langle f, g\rangle$ en lugar de $\langle P, f, g, Q\rangle$.

\subsection{Funtores y transformaciones naturales}

A continuación presentamos algunos conceptos y resultados básicos sobre funtores y transformaciones naturales, sobre los cuales estaremos trabajando a lo largo de este trabajo.

Damos por conocida la definición de categoría, funtor y la teoría básica de módulos.

La siguiente definición es motivada por el hecho de querer conectar dos funtores.

Definición 1.22. ( [1], Definición 6.1) Sean $F, G: \mathcal{A} \rightarrow \mathcal{B}$ funtores. Una transformación natural $\tau: F \rightarrow G$ es una función que asigna a cada objeto $A \in \mathcal{A}$ un morfismo $\tau_{A}: F(A) \rightarrow G(A)$ en $\mathcal{B}$, de tal manera que se satisface la siguiente condición de naturalidad: para cada morfismo $f: A \rightarrow A^{\prime}$ en $\mathcal{A}$ el siguiente diagrama conmuta:

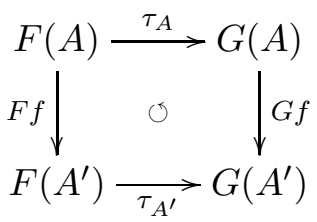

En este trabajo usaremos los siguientes funtores.

Ejemplo 1.23. Sea $\mathcal{A}$ una categoría. Entonces para cada objeto $A \in \mathcal{A}$, $\operatorname{Hom}_{\mathcal{A}}\left(A,_{-}\right): \mathcal{A} \rightarrow$ Set es un funtor definido como:

1. Para cada objeto $B \in \mathcal{A}, \operatorname{Hom}_{\mathcal{A}}\left(A,,_{-}\right)(B):=\operatorname{Hom}_{\mathcal{A}}(A, B)$.

2. Para un morfismo $f: B \rightarrow C$ en $\mathcal{A}, \operatorname{Hom}_{\mathcal{A}}\left(A,,_{-}\right)(f):=\operatorname{Hom}_{\mathcal{A}}(A, f)$, donde $\operatorname{Hom}_{\mathcal{A}}(A, f): \operatorname{Hom}_{\mathcal{A}}(A, B) \rightarrow \operatorname{Hom}_{\mathcal{A}}(A, C)$, tal que para $g:$ $A \rightarrow B$ en $\mathcal{A}, \operatorname{Hom}_{\mathcal{A}}(A, f)(g)=f \circ g$.

A dicho funtor se le llama funtor Hom covariante.

Ejemplo 1.24. Sea $\mathcal{A}$ una categoría. Entonces para cada objeto $A \in \mathcal{A}$, $\operatorname{Hom}_{\mathcal{A}}(-, A): \mathcal{A} \rightarrow$ Set es un funtor definido como: 
1. Para cada objeto $B \in \mathcal{A}, \operatorname{Hom}_{\mathcal{A}}(-, A)(B):=\operatorname{Hom}_{\mathcal{A}}(B, A)$.

2. Para un morfismo $f: B \rightarrow C$ en $\mathcal{A}, \operatorname{Hom}_{\mathcal{A}}(-, A)(f):=\operatorname{Hom}_{\mathcal{A}}(f, A)$, donde $\operatorname{Hom}_{\mathcal{A}}(f, A): \operatorname{Hom}_{\mathcal{A}}(C, A) \rightarrow \operatorname{Hom}_{\mathcal{A}}(B, A)$, tal que para $g:$ $C \rightarrow A$ en $\mathcal{A}, \operatorname{Hom}_{\mathcal{A}}(f, A)(g)=g \circ f$.

A dicho funtor se le llama funtor Hom contravariante.

Un ejemplo de transformación natural es el que sigue.

Ejemplo 1.25. Sean $A, A^{\prime} \in \mathcal{A} y \gamma: A \rightarrow A^{\prime}$ un morfismo en $\mathcal{A}$. Entonces $\tau_{\gamma}$ : $\operatorname{Hom}_{\mathcal{A}}\left(A^{\prime},_{-}\right) \rightarrow \operatorname{Hom}_{\mathcal{A}}\left(A,_{-}\right)$es una transformación natural, definida para cada $B \in \mathcal{A}$ como $\left(\tau_{\gamma}\right)_{B}: \operatorname{Hom}_{\mathcal{A}}\left(A^{\prime}, B\right) \rightarrow \operatorname{Hom}_{\mathcal{A}}(A, B)$, donde $\left(\tau_{\gamma}\right)_{B}(h)=h \circ \gamma$, para cada $h \in \operatorname{Hom}_{\mathcal{A}}\left(A^{\prime}, B\right)$.

Dualmente $\sigma_{\gamma}: \operatorname{Hom}_{\mathcal{A}}(-, A) \rightarrow \operatorname{Hom}_{\mathcal{A}}\left(-, A^{\prime}\right)$ es una transformación natural, definida para cada $B \in \mathcal{A}$ como $\left(\sigma_{\gamma}\right)_{B}: \operatorname{Hom}_{\mathcal{A}}(B, A) \rightarrow \operatorname{Hom}_{\mathcal{A}}\left(B, A^{\prime}\right)$, donde $\left(\sigma_{\gamma}\right)_{B}(h)=\gamma \circ h$, para cada $h \in \operatorname{Hom}_{\mathcal{A}}(B, A)$.

\section{Notación.}

1. Dado un funtor $F$, denotaremos por $1_{F}$ a la transformación natural identidad de $F$ en sí mismo.

2. Denotaremos por $1_{\mathcal{A}}$ al funtor identidad sobre la categoría $\mathcal{A}$.

Definición 1.26. ([1], Definición 6.5) Sean $F, G: \mathcal{A} \rightarrow \mathcal{B}$ funtores. Una transformación natural $\tau: F \rightarrow G$ se llama isomorfismo natural si $\tau_{A}$ es un isomorfismo para cada objeto $A \in \mathcal{A}$. En este caso se dice que $F$ y $G$ son isomorfos naturalmente y se denota como $F \cong G$.

Ejemplo 1.27. La transformación natural $\delta: 1_{R-M o d} \rightarrow \operatorname{Hom}\left(R,_{-}\right)$definida para cada $M \in R$-Mod como $\delta_{M}: M \rightarrow \operatorname{Hom}(R, M)$, donde $\left(\delta_{M}\right)(x):=\delta_{x}: R \rightarrow$ $M$ tal que $\delta_{x}(r)=r x$ para cada $x \in M$ y $r \in R$, es un isomorfismo natural.

Las siguientes definiciones muestran dos maneras de cómo componer dos transformaciones naturales. Una de ellas es usar la composición usual entre funtores y la otra es un poco más elaborada como veremos enseguida.

Definición 1.28. ([14], Definición 13.3) Sean $F, G, H: \mathcal{A} \rightarrow \mathcal{B}$ funtores $y$ $\tau: F \rightarrow G, \sigma: G \rightarrow H$ transformaciones naturales. Se define la composición $\sigma \circ \tau: F \rightarrow H$ para cada objeto $A \in \mathcal{A}$ como $(\sigma \circ \tau)_{A}: F(A) \rightarrow H(A)$, donde $(\sigma \circ \tau)_{A}:=\sigma_{A} \circ \tau_{A}$.

Definición 1.29. ([14], Definición 13.10) Sean $F, G: \mathcal{A} \rightarrow \mathcal{B}, H, K: \mathcal{B} \rightarrow$ $\mathcal{C}$ funtores y $\tau: F \rightarrow G, \sigma: H \rightarrow K$ transformaciones naturales. Se define el producto estrella $\sigma * \tau: H \circ F \rightarrow K \circ G$ para cada objeto $A \in \mathcal{A}$ como $(\sigma * \tau)_{A}: H F(A) \rightarrow K G(A)$, donde $(\sigma * \tau)_{A}:=K\left(\tau_{A}\right) \circ \sigma_{F(A)}=\sigma_{G(A)} \circ H\left(\tau_{A}\right)$. 
Observación 1.30. En algunas ocasiones a la transformación natural identidad $1_{F}$ sobre el funtor $F$, la denotaremos como $F$. En ese caso, para cada $A \in \mathcal{A}$ :

$$
(\sigma * F)_{A}=\sigma_{F(A)}
$$

y para cada $B \in \mathcal{B}$ :

$$
(F * \sigma)_{B}=F\left(\sigma_{B}\right) .
$$

\subsubsection{Equivalencias y adjunciones}

En este trabajo estudiaremos cierto tipo de retículas, las cuales se sabe que son isomorfas cuando tenemos una situación de equivalencia entre las categorías involucradas en esas retículas. Generalizaremos dicho resultado a una situación de adjunción.

A continuación presentamos los resultados que necesitaremos con respecto a la situación de equivalencia y adjunción entre categorías. Primero recordemos la siguiente definición.

Definición 1.31. ( [14], Definición 12.5 ) Sea $F: \mathcal{A} \rightarrow \mathcal{B}$ un funtor.

1. F es pleno, si la función inducida:

$$
F_{A, A^{\prime}}: \operatorname{Hom}_{\mathcal{A}}\left(A, A^{\prime}\right) \rightarrow \operatorname{Hom}_{\mathcal{B}}\left(F A, F A^{\prime}\right)
$$

es suprayectica para cada $A, A^{\prime} \in \mathcal{A}$.

2. F es fiel, si la función $F_{A, A^{\prime}}$ es inyectiva para cada $A, A^{\prime} \in \mathcal{A}$.

3. F es denso, si para cada $B \in \mathcal{B}$ existe $A \in \mathcal{A}$ tal que $F(A) \cong B$.

Para la demostración del siguiente teorema se requiere de un axioma de elección fuerte (para clases).

Teorema 1.32. ( [14], Teorema 14.11) Si $F: \mathcal{A} \rightarrow \mathcal{B}$ es un funtor, entonces las siguientes condiciones son equivalentes:

a) F es un funtor pleno, fiel y denso.

b) Existe un funtor $G: \mathcal{B} \rightarrow \mathcal{A}$, tal que $G F \cong 1_{\mathcal{A}}$ y $F G \cong 1_{\mathcal{B}}$.

c) Existe un funtor $G: \mathcal{B} \rightarrow \mathcal{A}$ e isomorfismos naturales $\varepsilon: F G \rightarrow 1_{\mathcal{B}}$ y $\eta: 1_{\mathcal{A}} \rightarrow G F$ tales que $F * \eta=(\varepsilon * F)^{-1}$ y $G * \varepsilon=(\eta * G)^{-1}$.

Definición 1.33. Un funtor $F: \mathcal{A} \rightarrow \mathcal{B}$ es una equivalencia si satisface alguno de los incisos del Teorema 1.32. En este caso se dice que las categorías $\mathcal{A}$ y $\mathcal{B}$ son equivalentes.

El Teorema 1.32, nos permite tener varios puntos de vista para una equivalencia.

Definición 1.34. Sean $R$ y $S$ dos anillos. Se dice que $R$ y $S$ son anillos Moritaequivalentes si las categorías $R$-Mod y $S$-Mod son equivalentes.

La situación de adjunción entre dos categorías se da a través de funtores que cumplen cierta condición que generaliza la situación de equivalencia. 
Teorema 1.35. ( [14], Teorema 27.9 ) Sean $F: \mathcal{A} \rightarrow \mathcal{B}$ y $G: \mathcal{B} \rightarrow \mathcal{A}$ funtores. Entonces las siguientes condiciones son equivalentes:

a) Existen transformaciones naturales $\varepsilon: F G \rightarrow 1_{\mathcal{B}}$ y $\eta: 1_{\mathcal{A}} \rightarrow G F$ (no necesariamente isomorfismos naturales) tales que $(\varepsilon * F) \circ(F * \eta)=1_{F}$ $y(G * \varepsilon) \circ(\eta * G)=1_{G}$.

b) Para cada par de objetos $A \in \mathcal{A}$ y $B \in \mathcal{B}$ existe una biyección $\varphi_{A, B}: \operatorname{Hom}_{\mathcal{B}}(F A, B) \rightarrow \operatorname{Hom}_{\mathcal{A}}(A, G B)$ la cual es natural en $A y$ $B$.

Definición 1.36. Una adjunción es una pareja $\langle F, G\rangle$ de funtores $F: \mathcal{A} \rightarrow \mathcal{B} y$ $G: \mathcal{B} \rightarrow \mathcal{A}$ que satisfacen alguno de los incisos del Teorema 1.35. En este caso se dice que $F: \mathcal{A} \rightarrow \mathcal{B}$ es un funtor adjunto izquierdo de $G: \mathcal{B} \rightarrow \mathcal{A}$ y que hay una situación de adjunción entre la categoría $\mathcal{A}$ y la categoría $\mathcal{B}$.

Obsérvese cómo la definición de funtor adjunto es un poco más general que la condición c) del Teorema 1.32.

En [2] la definición de funtor adjunto para categorías de módulos es presentada como en el inciso b) del Teorema 1.35.

En lo que sigue presentaremos algunas propiedades de los funtores adjuntos.

Recordemos las siguientes definiciones.

Definición 1.37. ( [14], Definición 6.2) Un morfismo $f: A \rightarrow B$ en una categoría $\mathcal{A}$, es un monomorfismo en $\mathcal{A}$ si para cada par de morfismos $h: C \rightarrow A y k:$ $C \rightarrow A$ en $\mathcal{A}$ que cumplen que $f \circ h=f \circ k$, se tiene que $h=k$. Es decir, si $f$ es cancelable por la izquierda con respecto a la composición en $\mathcal{A}$.

Definición 1.38. ( [14], Definición 6.9) Un morfismo $f: A \rightarrow B$ en una categoría $\mathcal{A}$, es un epimorfismo en $\mathcal{A}$ si para cada par de morfismos $h: B \rightarrow C$ y $k: B \rightarrow C$ en $\mathcal{A}$ que cumplen que $h \circ f=k \circ f$, se tiene que $h=k$. Es decir, si $f$ es cancelable por la derecha con respecto a la composición en $\mathcal{A}$.

Proposición 1.39. Sea $f: A \rightarrow B$ un morfismo en una categoría $\mathcal{A}$. Entonces las siguientes condiciones son equivalentes:

a) $f$ es un epimorfismo (monomorfismo).

b) Para todo $C \in \mathcal{A}$ el funtor contravariante (covariante)

$$
\begin{gathered}
\operatorname{Hom}_{\mathcal{A}}(f, C): \operatorname{Hom}_{\mathcal{A}}(B, C) \rightarrow \operatorname{Hom}_{\mathcal{A}}(A, C) \\
\left(\operatorname{Hom}_{\mathcal{A}}(C, f): \operatorname{Hom}_{\mathcal{A}}(C, A) \rightarrow \operatorname{Hom}_{\mathcal{A}}(C, B)\right)
\end{gathered}
$$

es una función inyectiva.

Proposición 1.40. Sea $F: \mathcal{A} \rightarrow \mathcal{B}$ un funtor adjunto izquierdo de $G: \mathcal{B} \rightarrow \mathcal{A}$. Entonces $F$ preserva epimorfismos y $G$ preserva monomorfismos. 
Demostración. Sea $f: A \rightarrow A^{\prime}$ un epimorfismo en $\mathcal{A}$. Por la Proposición 1.39, para probar que $F f: F A \rightarrow F A^{\prime}$ es un epimorfismo basta probar que $\operatorname{Hom}_{\mathcal{B}}(F f, B): \operatorname{Hom}_{\mathcal{B}}\left(F A^{\prime}, B\right) \rightarrow \operatorname{Hom}_{\mathcal{B}}(F A, B)$ es inyectiva para todo $B \in$ $\mathcal{B}$. Puesto que $F$ es adjunto izquierdo de $G$, por la Proposición 1.35 existen los isomorfismos $\varphi_{A, B}$ y $\varphi_{A^{\prime}, B}$ y se tiene el siguiente diagrama conmutativo:

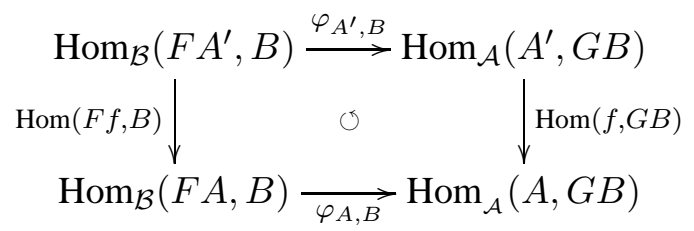

Por la Proposición 1.39, $\operatorname{Hom}(f, G B)$ es inyectiva y del diagrama anterior se concluye que $\operatorname{Hom}(F f, B)$ es inyectiva.

Análogamente se prueba que $G$ preserva monomorfismos.

Proposición 1.41. ( [14], Teorema 27.7 ) Sea $F: \mathcal{A} \rightarrow \mathcal{B}$ un funtor adjunto izquierdo de $G: \mathcal{B} \rightarrow \mathcal{A}$. Entonces $F$ preserva colímites y $G$ preserva límites.

Obsérvese que la Proposición 1.40 es un caso particular de la Proposición 1.41, ya que todo monomorfismo se puede ver como parte de un producto fibrado y todo epimorfismo como parte de un coproducto fibrado. Ver la Sección 21 del Capítulo VI de [14]

Presentamos la demostración de las siguientes propiedades que tienen los funtores $F$ y $G$ cuando son adjuntos, que son más específicas que las de la Proposición 1.41, porque es la situación que nos interesa en este trabajo.

Proposición 1.42. Sea $F: \mathcal{A} \rightarrow \mathcal{B}$ un funtor adjunto izquierdo de $G: \mathcal{B} \rightarrow \mathcal{A}$. Entonces $F$ preserva coproductos y $G$ preserva productos.

Demostración. Sea $\left\{A_{i}\right\}_{i \in I}$ una familia arbitraria de objetos en $\mathcal{A}$ y $\left\{\iota_{j}: A_{j} \rightarrow \bigoplus_{i \in I} A_{i}\right\}_{j \in I}$ un coproducto para dicha familia.

Veamos que $\left\{F\left(\iota_{j}\right): F\left(A_{j}\right) \rightarrow F\left(\bigoplus_{i \in I} A_{i}\right)\right\}_{j \in I}$ es un coproducto en $\mathcal{B}$ para la familia $\left\{F\left(A_{i}\right)\right\}_{i \in I}$. Sea $B \in \mathcal{B}$, basta probar que la función canónica inducida por dicha familia $\operatorname{Hom}_{\mathcal{B}}\left(F\left(\bigoplus_{i \in I} A_{i}\right), B\right) \rightarrow \prod_{i \in I} \operatorname{Hom}_{\mathcal{B}}\left(F\left(A_{i}\right), B\right)$ es una biyección. Puesto que $F$ es un adjunto izquierdo de $G$, por el Teorema 1.35 existe la biyección $\varphi_{\bigoplus_{i \in I}} A_{i}, B: \operatorname{Hom}_{\mathcal{B}}\left(F\left(\bigoplus_{i \in I} A_{i}\right), B\right) \rightarrow \operatorname{Hom}_{\mathcal{A}}\left(\bigoplus_{i \in I} A_{i}, G(B)\right)$.

En virtud de que $\bigoplus_{i \in I} A_{i}$ es un coproducto tenemos la biyección $\varphi^{\prime}: \operatorname{Hom}_{\mathcal{A}}\left(\bigoplus_{i \in I} A_{i}, G(B)\right) \rightarrow \prod_{i \in I} \operatorname{Hom}_{\mathcal{A}}\left(A_{i}, G(B)\right)$. Existe la biyección $\psi:$ $\prod_{i \in I} \operatorname{Hom}\left(A_{i}, G(B)\right) \rightarrow \prod_{i \in I} \operatorname{Hom}\left(F\left(A_{i}\right), B\right)$ como consecuencia del Teorema 1.35. Por lo tanto de la composición $\psi \circ \varphi^{\prime} \circ \varphi_{\bigoplus_{i \in I}} A_{i}, B$ se concluye el resultado. Análogamente se prueba que $G$ preserva productos. 


\subsection{Prerradicales}

En esta sección presentamos un breve resumen referente a la clase $R$-pr de todos los prerradicales sobre el anillo $R$. Veremos que $R$-pr es una gran retícula completa, así como otras propiedades que necesitaremos en este trabajo, donde nuestro principal objetivo es estudiar si hay alguna relación entre estas retículas cuando consideramos dos anillos diferentes. Lo referente a esta sección se puede consultar en [8].

En adelante, cuando hablemos de anillos nos estaremos refiriendo a anillos asociativos con 1 y consideraremos módulos izquierdos, pero los resultados también son válidos para módulos derechos.

Definición 1.43. ( [8], Definición 1 ) Un prerradical $\sigma$ sobre el anillo $R$ es una asignación $\sigma: R$-Mod $\rightarrow R$-Mod que cumple las siguientes condiciones:

1. Para todo $M \in R$-Mod, $\sigma(M) \leq M$.

2. Para todo homomorfismo $f: M \rightarrow N$ en R-Mod, $f(\sigma(M)) \leq \sigma(N)$.

Notemos que un prerradical $\sigma$ induce un funtor en la categoría $R$-Mod, de hecho, es un subfuntor del funtor identidad.

Ejemplo 1.44. Sean $\mathcal{C} \subseteq R$-Mod y $M \in R$-Mod. La Traza y el Rechazo, denotados respectivamente como $\operatorname{Tr}_{\mathcal{C}}\left(\_\right.$) y Rej $j_{\mathcal{C}}($ _) son prerradicales. La Traza y el Rechazo respecto a $\mathcal{C}$ se definen para cada $M \in R$-Mod de la manera siguiente:

$$
\operatorname{Tr}_{\mathcal{C}}(M):=\sum\{f(C) \mid f \in \operatorname{Hom}(C, M), C \in \mathcal{C}\}
$$

$y$

$$
\operatorname{Rej}_{\mathcal{C}}(M):=\bigcap\{\operatorname{ker} g \mid g \in \operatorname{Hom}(M, C), C \in \mathcal{C}\}
$$

El siguiente ejemplo es un caso particular del anterior.

Ejemplo 1.45. Sean $\mathbb{S}$ la clase de los $R$-módulos simples y $M \in R$-Mod. El Zoclo y el Radical de Jacobson, denotados respectivamente como Soc(_) y Rad(_) son prerradicales. El Zoclo y el Radical de Jacobson se definen para cada $M \in R$-Mod de la manera siguiente:

$$
\operatorname{Soc}(M)=\operatorname{Tr}_{\mathbb{S}}(M)=\sum\{N \leq M \mid N \text { es simple }\}
$$

$y$

$$
\operatorname{Rad}(M)=\operatorname{Rej}_{\mathbb{S}}(M)=\bigcap\{K<M \mid K \text { es un submódulo máximo }\}
$$

Observación 1.46. Sea $R$-simp un conjunto completo e irredundande de representantes de clases de isomorfismos de R-módulos simples. Notemos que para todo $M \in R$-Mod, $\operatorname{Tr}_{\mathbb{S}}(M)=\operatorname{Tr}_{R \text {-simp }}(M)$ y $\operatorname{Rej}_{\mathbb{S}}(M)=\operatorname{Rej}_{R-\operatorname{simp}}(M)$.

Se denota como $R$-pr a la clase de todos los prerradicales sobre el anillo $R$.

Las siguientes propiedades de los prerradicales con respecto a la suma directa y el producto directo se pueden ver en [11] y las usaremos en los siguientes capítulos. 
Proposición 1.47. ( [11], Proposición 2.2 ) Sean $\sigma \in R$-pr y $\left\{M_{\alpha}\right\}_{\alpha \in I} \subseteq R$-Mod. Entonces
1. $\sigma\left(\bigoplus_{\alpha \in I} M_{\alpha}\right)=\bigoplus_{\alpha \in I} \sigma\left(M_{\alpha}\right)$
2. $\sigma\left(\prod_{\alpha \in I} M_{\alpha}\right) \leq \prod_{\alpha \in I} \sigma\left(M_{\alpha}\right)$

En lo que sigue veremos que la clase $R$-pr tiene estructura de retícula completa.

Definición 1.48. Sean $\sigma, \tau \in R$-pr. Se dice que $\sigma \preceq \tau$ si $\sigma(M) \leq \tau(M)$ para todo $M \in R$-Mod.

Es claro que $\preceq$ es un orden parcial sobre la clase $R$-pr. Por lo tanto $\langle R$-pr, $\preceq\rangle$ es una clase parcialmente ordenada.

Proposición 1.49. Sean $\sigma, \tau \in R$-pr. Entonces existen el ínfimo y el supremo de $\sigma$ y $\tau$ en $R$-pr, denotados respectivamente como $\sigma \wedge \tau$ y $\sigma \vee \tau$. Los cuales se describen para cada $M \in R$-Mod como:

1. $(\sigma \wedge \tau)(M):=\sigma(M) \cap \tau(M)$.

2. $(\sigma \vee \tau)(M):=\sigma(M)+\tau(M)$.

Como consecuencia de la Definición 1.48 y de la Proposición 1.49, tenemos que $\langle R-p r, \preceq, \vee, \wedge\rangle$ es una gran ${ }^{3}$ retícula.

Se pueden describir el ínfimo y supremo arbitrarios en $R$-pr para cualquier subclase de $R$-pr como veremos en seguida.

Proposición 1.50. Sea $\left\{\sigma_{i}\right\}_{i \in \mathcal{C}} \subseteq R$-pr, donde $\mathcal{C}$ es una clase. Entonces existen el ínfimo y el supremo arbitarios, denotados respectivamente como $\bigwedge_{i \in \mathcal{C}} \sigma_{i} y \bigvee_{i \in \mathcal{C}} \sigma_{i}$. Los cuales se describen para cada $M \in R$-Mod como:

1. $\bigwedge_{i \in \mathcal{C}} \sigma_{i}(M):=\bigcap_{i \in \mathcal{C}} \sigma_{i}(M)$.

2. $\bigvee_{i \in \mathcal{C}}^{i \in \mathcal{C}} \sigma_{i}(M):=\sum_{i \in \mathcal{C}}^{i \in \mathcal{C}} \sigma_{i}(M)$.

La clase de submódulos de un módulo $M$, digamos $\left\{\sigma_{i}(M) \mid i \in \mathcal{C}\right\}$, es un conjunto. Por lo que podemos considerar un conjunto de índices $I$ tal que $\left\{\sigma_{i}(M) \mid\right.$ $i \in \mathcal{C}\}=\left\{\sigma_{i}(M) \mid i \in I\right\}$. Así que

$$
\bigcap_{i \in \mathcal{C}} \sigma_{i}(M)=\bigcap_{i \in I} \sigma_{i}(M)
$$

$\mathrm{y}$

$$
\sum_{i \in \mathcal{C}} \sigma_{i}(M)=\sum_{i \in I} \sigma_{i}(M)
$$

\footnotetext{
${ }^{3} \mathrm{El}$ término gran es porque $R$-pr es una clase que no necesariamente es un conjunto.
} 
1. PRELIMINARES

Concluimos entonces que $\langle R-p r, \preceq, \vee, \wedge\rangle$ es una gran retícula completa. El elemento menor y mayor en $R$-pr son respectivamente el funtor cero y el funtor identidad en $R$-Mod, los cuales denotaremos respectivamente como $0_{R}$ y $1_{R}$.

Sea $f: A \rightarrow B$ un homomorfismo y sean los submódulos $A^{\prime} \leq A$ y $B^{\prime} \leq B$. Entonces usaremos la siguientes notación:

$$
\begin{aligned}
& \overleftarrow{f}\left(B^{\prime}\right) \text { denotará la imagen inversa de } B^{\prime} \text { bajo } f \\
& \vec{f}\left(A^{\prime}\right) \text { denotará la imagen directa de } A^{\prime} \text { bajo } f
\end{aligned}
$$

La siguiente definición muestra dos tipos de prerradicales especiales.

Definición 1.51. Sean $M, N \in R$-Mod, tal que $N \leq M$. Definimos los siguientes prerradicales para toda $K \in R$-Mod:

1. $\alpha_{N}^{M}(K):=\sum\{f(N) \mid f: M \rightarrow K\}$.

2. $\omega_{N}^{M}(K):=\bigcap\{\overleftarrow{g}(N) \mid g: K \rightarrow M\}$

A estos prerradicales se les llama prerradicales alfa y omega respectivamente.

La definición anterior también la podemos encontrar en [4] pero con una notación distinta.

Observación 1.52. Notemos que si $\{M\}=\mathcal{C} \subseteq R$-Mod, entonces

$$
\alpha_{M}^{M}\left(\_\right)=\operatorname{Tr}_{\mathcal{C}}\left(\_\right)=\operatorname{Tr}_{M}\left(\_\right)
$$

$y$

$$
\omega_{0}^{M}\left(\_\right)=\operatorname{Rej} j_{\mathcal{C}}\left(\_\right)=\operatorname{Rej}_{M}\left(\_\right)
$$

$$
\text { Si } \mathcal{C} \subseteq R \text {-Mod, entonces } \operatorname{Tr}_{\mathcal{C}}\left(\_\right)=\bigvee_{M \in \mathcal{C}} \alpha_{M}^{M} y \operatorname{Rej} j_{\mathcal{C}}\left(\_\right)=\bigwedge_{M \in \mathcal{C}} \omega_{0}^{M} .
$$

Definición 1.53. ( [12] ) Sean $M \in R$-Mod y $N \leq M$. Se dice que $N$ es un submódulo totalmente invariante de $M$ si para cada $f \in \operatorname{Hom}_{R}(M, M)$ se tiene que $f(N) \leq N$.

Observación 1.54. Notemos que si $N$ es un submódulo totalmente invariante de $M$, entonces $\alpha_{N}^{M}(M)=N y \omega_{N}^{M}(M)=N$

La siguiente proposición da una caracterización de los submódulo totalmente invariantes en términos de prerradicales.

Proposición 1.55. Sean $M \in R$-Mod y $N \leq M$. Entonces $N$ es un submódulo totalmente invariante de $M$ si y sólo si existe $\sigma \in R$-pr tal que $\sigma(M)=N$.

Proposición 1.56. ( $[8]$, Proposición 5 ) Sean $\sigma \in R$-pr, $M \in R$-Mod y $N$ un submódulo totalmente invariante de $M$. Entonces $\sigma(M)=N$ si y sólo si $\alpha_{N}^{M} \preceq$ $\sigma \preceq \omega_{N}^{M}$. 
Observación 1.57. De la proposición 1.56, notamos que si $N$ es un submódulo totalmente invariante de $M$, entonces $\alpha_{N}^{M} y \omega_{N}^{M}$ son respectivamente el menor $y$ mayor prerradical que asignan a $M$ el submódulo $N$.

Notemos que $\sigma(M)$ es un submódulo totalmente invariante de $M$ para todo $\sigma \in R$-pr y $M \in R$-Mod. El siguiente resultado muestra la importancia de los prerradicales alfa y omega pues dice que cada prerradical en $R$-pr se puede escribir en términos de dichos prerradicales.

Teorema 1.58. ( [10], Sección 1.1 ) Sea $\sigma \in R$-pr. Entonces

$$
\sigma=\bigvee\left\{\alpha_{\sigma M}^{M} \mid M \in R-\operatorname{Mod}\right\}=\bigwedge\left\{\omega_{\sigma M}^{M} \mid M \in R-\operatorname{Mod}\right\}
$$

La siguiente proposición no es difícil de verificar usando las definiciones de los prerradicales alfa y omega.

Proposición 1.59. ( [10], Proposición 1.2 ) Sean $\left\{M_{i}\right\}_{i \in I},\left\{N_{i}\right\}_{i \in I} \subseteq R$-Mod. Entonces:

1. Si $M=\bigoplus_{i \in I} M_{i}, N=\bigoplus_{i \in I} N_{i}$ y $N$ es un submódulo totalmente invariante de $M$, se tiene que $\bigvee_{i \in I} \alpha_{N_{i}}^{M_{i}}=\alpha_{N}^{M}$.

2. Si $M=\prod_{i \in I} M_{i}, N=\prod_{i \in I} N_{i}$ y $N$ es un submódulo totalmente invariante de $M$, se tiene que $\bigwedge_{i \in I} \omega_{N_{i}}^{M_{i}}=\omega_{N}^{M}$.

Los prerradicales alfa y omega también aparecen en la caracterización de los átomos y coátomos de la retícula $R$-pr.

Teorema 1.60. ( [8], Teorema 7 ) Sea $\sigma \in R$-pr. Entonces R-pr es una (gran) retícula atómica y coatómica. Donde el conjunto de átomos es

$$
\left.\left\{\alpha_{T}^{E T} \mid T \in R \text {-Simp (ET es la cápsula inyectiva de } T\right)\right\}
$$

y el conjunto de coátomos es

$$
\left\{\omega_{I}^{R} \mid I \text { ideal máximo de } R\right\} .
$$

\subsubsection{Prerradicales idempotentes $y$ radicales}

En este trabajo daremos algunos resultados que involucran a los prerradicales idempotentes y a los radicales. Esta clase de prerradicales se definen con base en las siguientes operaciones binarias en $R$-pr.

Definición 1.61. ( [8], Definición 2 ) Sean $\sigma, \tau \in R$-pr. Se define el producto $\sigma \cdot \tau$, como

$(\sigma \cdot \tau)(M)=(\sigma \tau)(M)=\sigma(\tau(M))$, para cada $M \in R$-Mod.

Es decir, el producto de prerradicales es la composición de funtores. 
Definición 1.62. ( [8], Definición 2 ) Sean $\sigma, \tau \in R$-pr. Se define el coproducto $(\sigma: \tau)$ para cada $M \in R$-mod, como el submódulo $(\sigma: \tau)(M)$ de $M \in$ tal que $(\sigma: \tau)(M) / \sigma(M)=\tau(M / \sigma(M))$.

Para tener más clara la definición del coproducto, consideremos la siguiente suceción exacta corta, donde $i: \sigma(M) \hookrightarrow M$ y $p: M \rightarrow M / \sigma(M)$ son la inclusión y la proyección canónica respectivamente:

$$
0 \longrightarrow \sigma(M) \stackrel{i}{\longrightarrow} M \stackrel{p}{\longrightarrow} M / \sigma(M) \longrightarrow 0
$$

Entonces $(\sigma: \tau)(M)=\overleftarrow{p}(\tau(M / \sigma(M)))$

El producto y el coproducto de prerradicales preservan orden y son asociativos. Además para $\sigma, \tau \in R$-pr tenemos las siguientes desigualdades

$$
\sigma \tau \preceq \sigma \wedge \tau \preceq \sigma, \tau \preceq \sigma \vee \tau \preceq(\sigma: \tau)
$$

Se tienen ciertas propiedades para estas operaciones.

Teorema 1.63. ( [8], Teorema 8 ) Sean $\sigma, \tau, \eta \in R$-pr, $\left\{\sigma_{i}\right\}_{i \in I} \subseteq R$-pry $M \in R$ Mod. Se tienen las siguientes propiedades

1. (Ley Modular ) Si $\sigma \preceq \tau$, entonces $\sigma \vee(\tau \wedge \eta)=\tau \wedge(\sigma \vee \eta)$

2. $\left(\bigwedge_{i \in I} \sigma_{i}\right) \tau=\bigwedge_{i \in I}\left(\sigma_{i} \tau\right)$

3. $\left(\bigvee_{i \in I} \sigma_{i}\right) \tau=\bigvee_{i \in I}\left(\sigma_{i} \tau\right)$

4. $\left(\tau: \bigvee_{i \in I} \sigma_{i}\right)=\bigvee_{i \in I}\left(\tau: \sigma_{i}\right)$

5. $\left(\tau: \bigwedge_{i \in I} \sigma_{i}\right)=\bigwedge_{i \in I}\left(\tau: \sigma_{i}\right)$

6. $(\tau: \eta) \sigma \preceq(\tau \sigma: \eta \sigma)$

7. $(\sigma: \tau)(\sigma: \eta) \preceq(\sigma: \tau \eta)$

Definición 1.64. ( [19], Página 136 ) Sea $\sigma \in R$-pr. Se dice que $\sigma$ es un prerradical idempotente si es idempotente con respecto al producto, es decir, si $\sigma \sigma=\sigma$.

Definición 1.65. ( [19], Página 136 ) Sea $\sigma \in R$-pr. Se dice que $\sigma$ es radical si es idempotente con respecto al coproducto, es decir, si $(\sigma: \sigma)=\sigma$.

Observación 1.66. Notemos que $\sigma \in R$-pr es radical si y sólo si para todo $M \in$ $R$-Mod se tiene que $\sigma(M / \sigma(M))=0$. Puesto que si $\sigma$ es radical, entonces

$$
\sigma(M / \sigma(M))=(\sigma: \sigma)(M) / \sigma(M)=\sigma(M) / \sigma(M)=0 .
$$

En el Teorema 1.63, cuando $\sigma$ es radical se da la igualdad de la parte 6 y cuando $\sigma$ es idempotente se da la igualdad de 7 . 
Ejemplo 1.67. Sea $M \in R$-Mod. Entonces $\alpha_{M}^{M}$ es un prerradical idempotente $y$ $\omega_{0}^{M}$ es un radical.

En adelante, denotaremos a la clase de los prerradicales idempotentes como $R$-idem y a la clase de los radicales como $R$-rad.

Por los incisos 3 y 5 de la Proposición 1.63 se tiene el siguiente resultado:

Proposición 1.68. Sea $\left\{\sigma_{i}\right\}_{i \in I} \subseteq R$-pr. Entonces

1. Si $\left\{\sigma_{i}\right\}_{i \in I} \subseteq R$-idem, entonces $\bigvee_{i \in I} \sigma_{i} \in R$-idem.

2. Si $\left\{\sigma_{i}\right\}_{i \in I} \subseteq R$-rad, entonces $\bigwedge_{i \in I} \sigma_{i} \in R$-rad.

De la Observación 1.52, Ejemplo 1.67 y la Proposición anterior se tiene que si $\mathcal{C} \subseteq R$-Mod, entonces $\bigvee_{M \in \mathcal{C}} \alpha_{M}^{M}=\operatorname{Tr} r_{\mathcal{C}}\left(\_\right) \in R$-idem y $\bigwedge_{M \in \mathcal{C}} \omega_{0}^{M}=R e j_{\mathcal{C}}\left(\_\right) \in R$ rad. Mas aún, el siguiente resultado muestra como son los prerradicales idempotentes y radicales.

Proposición 1.69. Sea $\sigma \in R$-pr. Entonces

1. $\sigma \in R$-idem si y sólo si $\sigma=\bigvee\left\{\alpha_{M}^{M} \mid \sigma(M)=M, M \in R\right.$-Mod $\}$.

2. $\sigma \in R$-rad si y sólo si $\sigma=\bigwedge\left\{\omega_{0}^{M} \mid \sigma(M)=0, M \in R\right.$-Mod $\}$

De lo anterior tenemos el siguiente ejemplo.

Ejemplo 1.70. Notemos que del Ejemplo 1.45 y de la Observación 1.52 el Zoclo es un prerradical idempotente y el Radical de Jacobson es un prerradical radical, puesto que Soc $\left(\_\right)=\bigvee_{S \in R \text {-simp }} \alpha_{S}^{S} y \operatorname{Rad}\left(\mathbf{Z}^{\prime}\right)=\bigwedge_{S \in R \text {-simp }} \omega_{0}^{S}$.

Además para $\mathcal{S} \subseteq R$-simp, se puede definir el zoclo relativo y el radical de

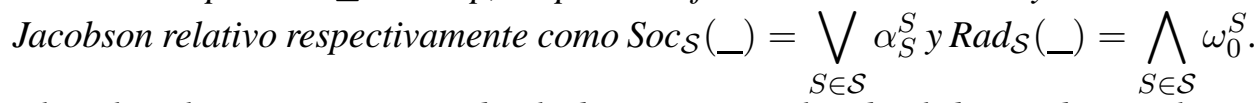
El zoclo relativo es un prerradical idempotente y el radical de Jacobson relativo es radical.

Dado $\sigma \in R$-pr, se definen las siguientes clases de $R$-módulos.

$$
\mathbb{T}_{\sigma}=\{M \in R \text {-Mod } \mid \sigma(M)=M\}
$$

y

$$
\mathbb{F}_{\sigma}=\{M \in R \text {-Mod } \mid \sigma(M)=0\}
$$

La clase $\mathbb{T}_{\sigma}$ es una clase de pretorsión, es decir, es una clase de $R$-módulos que es cerrada bajo epimorfismos y sumas directas. Dualmente la clase $\mathbb{F}_{\sigma}$ es una clase pre-libre de torsión, es decir, es una clase de $R$-módulos que es cerrada bajo monomorfsmos y productos directos.

Dados $\sigma, \tau \in R$-pr. Si $\sigma \preceq \tau$, entonces $\mathbb{T}_{\sigma} \subseteq \mathbb{T}_{\tau}$ y $\mathbb{F}_{\sigma} \supseteq \mathbb{F}_{\tau}$. Puesto que si $M \in \mathbb{T}_{\sigma}$, se tiene que $M=\sigma(M) \leq \tau(M)$. Dualmente si $M \in \mathbb{F}_{\tau}$, se tiene que $0=\tau(M) \geq \sigma(M)$, de donde $\sigma(M)=0$. 
Los siguientes conceptos se pueden encontrar en [19], páginas 137 y 157. En adelante, denotaremos a la clase de todos los ordinales como $\mathcal{O R}$. Para un prerradical, se puede definir sus potencias.

Definición 1.71. Sean $\sigma \in R$-pr y $\lambda \in \mathcal{O} \mathcal{R}$. El prerradical $\sigma^{\lambda}$ se define recursivamente como $\sigma^{0}=1_{R}, \sigma^{1}=\sigma, \sigma^{2}=\sigma \sigma, \cdots, \sigma^{\lambda+1}=\sigma \sigma^{\lambda}$. Si $\gamma$ es un ordinal limite, $\sigma^{\gamma}:=\bigwedge\left\{\sigma^{\lambda} \mid \lambda \in \mathcal{O R}, \lambda<\gamma\right\}$

Notemos que para $\gamma, \lambda \in \mathcal{O} \mathcal{R}$ tales que $\lambda \leq \gamma$, se tiene que $\sigma^{\gamma} \preceq \sigma^{\lambda}$. Por lo que se puede considerar el prerradical $\widehat{\sigma}:=\bigwedge\left\{\sigma^{\lambda} \mid \lambda \in \mathcal{O} \mathcal{R}\right\}$, el cual resulta el mayor prerradical idempotente menor o igual que $\sigma$.

Proposición 1.72. Sean $\sigma \in R$-pr y $\widehat{\sigma}=\bigwedge\left\{\sigma^{\lambda} \mid \lambda \in \mathcal{O} \mathcal{R}\right\} \in R$-pr. Entonces se cumple:

1. $\widehat{\sigma} \preceq \sigma$.

2. $\widehat{\sigma} \in R$-idem.

3. Si $\tau \in R$-idem, $\tau \preceq \sigma$, entonces $\tau \preceq \widehat{\sigma}$.

Dualmente, se pueden definir las copotencias de un prerradical.

Definición 1.73. Sean $\sigma \in R$-pr y $\lambda \in \mathcal{O} \mathcal{R}$. El prerradical $\sigma_{\lambda}$ se define recursivamente como $\sigma_{0}=0_{R}, \sigma_{1}=\sigma, \sigma_{2}=(\sigma: \sigma), \cdots, \sigma_{\lambda+1}=\left(\sigma_{\lambda}: \sigma\right)$. Si $\gamma$ es un ordinal limite, $\sigma_{\gamma}:=\bigvee\left\{\sigma_{\lambda} \mid \lambda \in \mathcal{O} \mathcal{R}, \lambda<\gamma\right\}$.

Observemos que para $\gamma, \lambda \in \mathcal{O} \mathcal{R}$ tal que $\lambda \leq \gamma$, en este caso se tiene que $\sigma_{\gamma} \succeq \sigma_{\lambda}$. Así que podemos considerar el prerradical $\bar{\sigma}:=\bigvee\left\{\sigma_{\lambda} \mid \lambda \in \mathcal{O R}\right\}$, el cual resulta el menor radical mayor o igual que $\sigma$.

Proposición 1.74. Sean $\sigma \in R$-pr y $\bar{\sigma}:=\bigvee\left\{\sigma_{\lambda} \mid \lambda \in \mathcal{O} \mathcal{R}\right\} \in R$-pr. Entonces se cumple:

1. $\bar{\sigma} \succeq \sigma$.

2. $\bar{\sigma} \in R$-rad.

3. Si $\tau \in R$-rad, $\tau \succeq \sigma$, entonces $\tau \succeq \bar{\sigma}$.

Observación 1.75. Si $\sigma \in R$-idem, entonces $\sigma_{\lambda} \in R$-idem, para toda $\lambda \in \mathcal{O} \mathcal{R}$. Por lo que si $\sigma \in R$-idem, entonces $\bar{\sigma} \in R$-idem, es decir, $\bar{\sigma}$ es un prerradical radical idempotente.

Dualmente, si $\sigma \in R$-rad, entonces $\sigma^{\lambda} \in R$-rad, para toda $\lambda \in \mathcal{O} \mathcal{R}$. Por lo que si $\sigma \in R$-rad, entonces $\widehat{\sigma} \in R$-rad, es decir, $\widehat{\sigma}$ es un radical idempotente

Proposición 1.76. Sea $\sigma \in R$-pr. Entonces $\mathbb{T}_{\sigma}=\mathbb{T}_{\widehat{\sigma}} y \mathbb{F}_{\sigma}=\mathbb{F}_{\bar{\sigma}}$.

En lo que sigue presentaremos algunas clases de prerradicales en términos de los prerradicales alfa y omega que estaremos usarando a lo largo de este trabajo.

Notemos que para $\sigma \in R$-pr, $\sigma(R)$ es un ideal (bilateral) de $R$. Por la Proposición 1.55 , si $I$ es un $R$-submódulo, entonces $I$ es un submódulo totalmente invariante de $R$, si y sólo si, $I$ es un ideal (bilateral).

Proposición 1.77. Sea $\sigma \in R$-pr. Las siguientes afirmaciones son equivalentes:

1. $\sigma$ preserva epimorfismos. 
2. Para todo $M \in R$-Mod, $\sigma(M)=\sigma(R) M$.

3. $\sigma=\alpha_{I}^{R}$ con I un ideal de $R$.

Podemos observar que los prerradicales $\sigma=\alpha_{I}^{R}$ de la Proposición 1.77 son Radicales, puesto que $\sigma$ preserva epimorfismos.

Definición 1.78. Un t-radical es un prerradical que cumple alguna de las afirmaciones de la Proposición 1.77.

Definición 1.79. Un prerradical es exacto izquierdo si como funtor es exacto izquierdo.

Proposición 1.80. Sea $\sigma \in R$-pr. Entonces las siguientes afirmaciones son equivalentes:

1. $\sigma$ es exacto izquierdo.

2. Para todo $M \in R$-Mod y $N \leq M, \sigma(N)=N \cap \sigma(M)$.

3. $\sigma$ es idempotente y $\mathbb{T}_{\sigma}$ es cerrada bajo monomorfismos.

Definición 1.81. ( [9], Definición 3.1 ) Sea $\sigma \in R$-pr. Se definen los siguientes prerradicales:

1. $e(\sigma)=\bigwedge\{\eta \in R-p r \mid \eta \sigma=\sigma\}$.

2. $a(\sigma)=\bigvee\{\eta \in R-p r \mid \eta \sigma=0\}$

3. $c(\sigma)=\bigvee\{\eta \in R-p r \mid(\sigma: \eta)=\sigma\}$

4. $t(\sigma)=\bigwedge\{\eta \in R-p r \mid(\sigma: \eta)=1\}$

A tales prerradicales se les llama el igualador, el anulador, el coigualador y totalizador de $\sigma$ respectivamente.

Es fácil ver que $e(\sigma) \sigma=\sigma, a(\sigma) \sigma=0,(\sigma: c(\sigma))=\sigma \mathrm{y}(\sigma: t(\sigma))=1$. Se tiene también que $e(\sigma)$ es un prerradical idempotente, $a(\sigma)$ y $c(\sigma)$ son radicales y $t(\sigma)$ es un prerradical exacto izquierdo.

El siguiente resultado muestra algunas propiedades para los prerradicales anteriores.

Teorema 1.82. ( [9], Teorema 3.1) Sea $\sigma \in R$-pr. Entonces se tienen las siguientes propiedades:

a) $\sigma \preceq e(\sigma)$

b) $e(\sigma)=\sigma$ si y sólo si $\sigma$ es prerradical idempotente.

c) $e(\sigma)=\bigvee\left\{\alpha_{\sigma M}^{\sigma M} \mid M \in R-\operatorname{Mod}\right\}$

d) $a(\sigma)=\bigwedge\left\{\omega_{0}^{\sigma M} \mid M \in R\right.$-Mod $\}$

e) $\sigma \succeq c(\sigma)$

f) $c(\sigma)=\sigma$ si y sólo si $\sigma$ es un radical.

g) $c(\sigma)=\bigwedge\left\{\omega_{0}^{M / \sigma M} \mid M \in R\right.$-Mod $\}$

h) $t(\sigma)=\bigvee\left\{\alpha_{M}^{M} \mid M \in R-\operatorname{Mod}, \sigma(R) M=0\right\}$

Lema 1.83. Sea I un ideal de $R$. Entonces

1. $t\left(\alpha_{I}^{R}\right)(M)=\{x \in M \quad \mid \quad I x=0\}$, para cada $M \in R$-Mod. 
2. $\alpha_{I}^{R}=a\left(t\left(\alpha_{I}^{R}\right)\right)$

Lema 1.84. Sea I un ideal de R. Entonces $t\left(\alpha_{I}^{R}\right)=\alpha_{R / I}^{R / I}$.

\subsubsection{Particiones de $R$-pr inducida por una clase de módu- los}

Más adelante ( Capítulo 4 ) estudiaremos ciertas particiones de la retícula $R$-pr inducidas por un ideal del anillo $R$. Por lo que en esta sección recordaremos una partición de $R$-pr la cual usaremos en su momento. Los siguientes resultados se pueden consultar en [10].

Definición 1.85. Sea $\mathcal{C}$ una clase de $R$-Mod y $\sigma, \tau \in R$-pr. Diremos que $\sigma \sim_{\mathcal{C}} \tau$ si $\sigma M=\tau M$, para cada $M \in \mathcal{C}$.

Para $\mathcal{C} \subseteq R$-Mod, $\sim_{\mathcal{C}}$ es una relación de equivalencia en $R$-pr. Denotaremos a la clase de equivalencia de $\tau \in R$-pr respecto a $\sim_{\mathcal{C}}$ como $[\tau]_{\mathcal{C}}$.

Definición 1.86. Dados $\sigma, \tau \in R$-pr, se puede definir el intervalo:

$$
[\sigma, \tau]:=\{\gamma \in R-p r \mid \sigma \preceq \gamma \preceq \tau\} .
$$

Proposición 1.87. Sea $\mathcal{C} \subseteq R$-Mod y $\tau \in R$-pr. Entonces

$$
[\tau]_{\mathcal{C}}=\left[\bigvee_{M \in \mathcal{C}} \alpha_{\tau M}^{M}, \bigwedge_{M \in \mathcal{C}} \omega_{\tau M}^{M}\right] .
$$




\section{Capítulo 2}

\section{Conexiones entre retículas de prerradi- cales}

Como hemos mencionado antes, en este trabajo estudiaremos la relación que puede haber entre las retículas de prerradicales $R$-pr y $S$-pr cuando los anillos $R$ y $S$ están conectados de alguna manera. Una motivación a esto es la Proposición I.9.2 de [4] que también es la Proposición 5.2 que aparece en [3], en donde se demuestra que dichas retículas son isomorfas cuando los anillos $R$ y $S$ son Moritaequivalentes, es decir, cuando las categorías $R$-Mod y $S$-Mod son equivalentes. Con base en lo anterior abarcaremos el caso más general en el que hay una situación de adjunción entre las categorías $R$-Mod y $S$-Mod y probaremos que en este caso hay una conexión de Galois entre las retículas de prerradicales respectivas. De hecho, este caso resulta ser aún más general puesto que el Teorema de Watts-Eilenberg, ver [5] y [20], caracteriza a los pares adjuntos y en este trabajo daremos varias maneras de cómo describir a la conexión de Galois inducida por dicha adjunción.

\subsection{Las retículas $R$-pr y $S$-pr cuando $R$ y $S$ son Morita- equivalentes}

En esta sección detallaremos la prueba de la Proposición I.9.2 (5.2 de [3]) que enuncian Bican, Jambor, Kepka y Nẽmec en [4]. Dicha Proposición, en este trabajo la presentaremos como el Teorema 2.22, cuya demostración se obtendrá de varios resultados previos definiendo ciertas asignaciones entre la retícula de prerradicales y una clase cuyos miembros son endofuntores y transformaciones naturales. Por lo que este camino para probar la Proposición I.9.2, es un tanto distinto al que aparece en las referencias mencionadas. Es importante señalar que en [4] se usa una notación muy distinta a la nuestra y sólo aparece un esbozo de la demostración.

Primero definiremos la siguiente clase.

Definición 2.1. Sea $\mathcal{A}$ una categoría. Consideremos las siguientes parejas $(H, \mu)$, donde:

a) $H: \mathcal{A} \rightarrow \mathcal{A}$ es un funtor $y$

b) $\mu: H \rightarrow 1_{\mathcal{A}}$ es un monomorfismo natural.

Se define la clase $\operatorname{Pr}(\mathcal{A})$ que consta de los miembros $(H, \mu)$.

Ahora veamos algunas características de la clase $\operatorname{Pr}(\mathcal{A})$. 
Lema 2.2. Sea $(H, \mu) \in \operatorname{Pr}(\mathcal{A})$. Entonces $H$ preserva monomorfismos.

Demostración. Sea $f: A \rightarrow A^{\prime}$ un morfismo en la categoría $\mathcal{A}$. Supongamos que $f$ es un monomorfismo y veamos que $H(f): H(A) \rightarrow H\left(A^{\prime}\right)$ es un monomorfismo en la categoría $\mathcal{A}$. Se tiene el siguiente diagrama conmutativo:

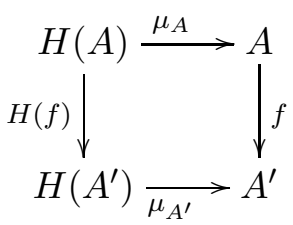

De donde, $\mu_{A^{\prime}} \circ H(f)=f \circ \mu_{A}$ y $\mu_{A^{\prime}} \circ H(f)$ es un monomorfismo puesto que $f \circ \mu_{A}$ es un monomorfismo. Por lo tanto $H(f)$ es un monomorfismo.

En lo que sigue veremos que la clase $\operatorname{Pr}(\mathcal{A})$ tiene estructura de pre-orden.

Definición 2.3. Sean $(H, \mu),(K, v) \in \operatorname{Pr}(\mathcal{A})$. Diremos que $(H, \mu) \leq(K, v)$ si $y$ sólo si para cada $A \in \mathcal{A}$ existe un triángulo conmutativo:

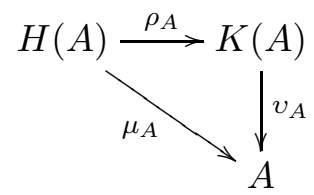

En efecto, $\leq$ es un pre-orden sobre la clase $\operatorname{Pr}(\mathcal{A})$.

Lema 2.4. Sean $(H, \mu),(K, v) \in \operatorname{Pr}(\mathcal{A})$, tales que $(H, \mu) \leq(K, v)$. Entonces $\rho: H \rightarrow K$ tal que para cada $A \in \mathcal{A}, \rho_{A}: H(A) \rightarrow K(A)$ es como el del Diagrama (4), es un monomorfismo natural.

Demostración. Sea $f: A \rightarrow A^{\prime}$ un morfismo en la categoría $\mathcal{A}$. Entonces veamos que el siguiente diagrama es conmutativo:

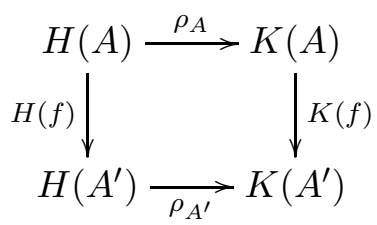

Puesto que $(H, \mu) \leq(K, v)$ y $v$ es una transformación natural, tenemos el siguiente diagrama conmutativo:

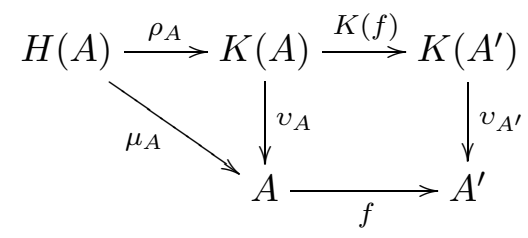

de donde,

$$
v_{A^{\prime}} \circ K(f) \circ \rho_{A}=f \circ \mu_{A} .
$$


Como $\mu$ es una transformación natural, $\mu_{A^{\prime}} \circ H(f)=f \circ \mu_{A}$. Para $A^{\prime} \in \mathcal{A}$, por el preorden $\leq$, se tiene que $\mu_{A^{\prime}}=v_{A^{\prime}} \circ \rho_{A^{\prime}}$. Así, la igualdad (6) queda como sigue:

$$
v_{A^{\prime}} \circ K(f) \circ \rho_{A}=\mu_{A^{\prime}} \circ H(f)=v_{A^{\prime}} \circ \rho_{A^{\prime}} \circ H(f),
$$

de ahí, obtenemos que $K(f) \circ \rho_{A}=\rho_{A^{\prime}} \circ H(f)$, ya que $v_{A^{\prime}}$ es un monomorfismo. Por lo tanto, $\rho$ es una transformación natural. Por último, como $(H, \mu) \leq(K, v)$, para cada $A \in \mathcal{A}$, es claro que $\rho_{A}$ es un monomorfismo.

De aquí en adelante consideraremos una categoría $\mathcal{X}$ equivalente a la categoría $\mathcal{A}$. Por lo que existen los funtores $F: \mathcal{A} \rightarrow \mathcal{X}$ y $G: \mathcal{X} \rightarrow \mathcal{A}$ y los isomorfismos naturales $\eta: 1_{\mathcal{A}} \rightarrow G F$ y $\varepsilon: F G \rightarrow 1_{\mathcal{X}}$, con los que estaremos trabajando en esta sección. Obsérvese que para cada $A \in \mathcal{A}$, podemos considerar el morfismo $\eta_{A}^{-1}$ puesto que $\eta$ es un isomorfismo natural.

Proposición 2.5. Sean $\mathcal{X}$ y A categorías equivalentes. Las siguientes asignaciones están bien definidas y preservan orden:

a) $\Phi: \operatorname{Pr}(\mathcal{A}) \rightarrow \operatorname{Pr}(\mathcal{X})$ tal que para cada $(H, \mu) \in \operatorname{Pr}(\mathcal{A}), \Phi(H, \mu)=$ $(F H G, \varepsilon \circ F \mu G)$.

b) $\Psi: \operatorname{Pr}(\mathcal{X}) \rightarrow \operatorname{Pr}(\mathcal{A})$ tal que para cada $(K, v) \in \operatorname{Pr}(\mathcal{X}), \Psi(K, v)=$ $\left(G K F, \eta^{-1} \circ G v F\right)$.

Demostración. a) Para ver que la asignación $\Phi$ está bien definida, basta ver que para cada $X \in \mathcal{X},(\varepsilon \circ F \mu G)_{X}: F H G(X) \rightarrow X$ es un monomorfismo. Lo cual es cierto, pues como $\varepsilon$ es un isomorfismo natural, se tiene que $\varepsilon_{X}$ es un monomorfismo para cada $X \in \mathcal{X}$. También $F\left(\mu_{G(X)}\right)$ es un monomorfismo ya que $\mu_{G(X)}$ es un monomorfismo y el funtor $F$ preserva monomorfismos. Por lo tanto, $\varepsilon_{X} \circ F\left(\mu_{G(X)}\right)$ es un monomorfismo.

Ahora veamos que la asignación $\Phi$ preserva orden. Sean $(H, \mu),\left(H^{\prime}, \mu^{\prime}\right) \in$ $\operatorname{Pr}(\mathcal{A})$ tales que $(H, \mu) \leq\left(H^{\prime}, \mu^{\prime}\right)$. Entonces veamos que $\phi(H, \mu) \leq \phi\left(H^{\prime}, \mu^{\prime}\right)$, es decir, mostremos que $(F H G, \varepsilon \circ F \mu G) \leq\left(F H^{\prime} G, \varepsilon \circ F \mu^{\prime} G\right)$. Sea $X \in \mathcal{X}$. Como $(H, \mu) \leq\left(H^{\prime}, \mu^{\prime}\right)$, para $G(X) \in \mathcal{A}$ existe el siguiente diagrama conmutativo:

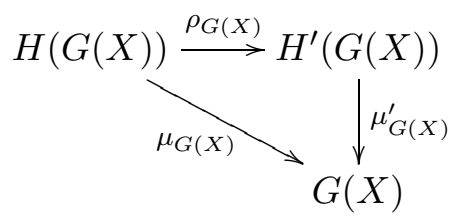


Aplicando el funtor $F$ al diagrama anterior, obtenemos el siguiente diagrama conmutativo:

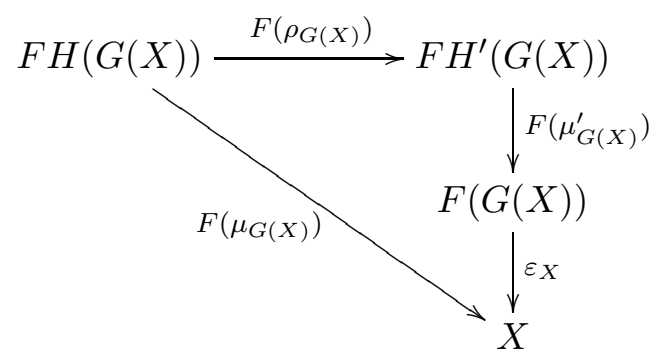

Del último diagrama y de la Definición 2.3, concluimos que $\Phi(H, \mu) \leq \Phi\left(H^{\prime}, \mu^{\prime}\right)$.

Para el inciso b), la prueba es dual a la del inciso a).

Definición 2.6. Sean $(H, \mu),\left(H^{\prime}, \mu^{\prime}\right) \in \operatorname{Pr}(\mathcal{A})$. Diremos que $(H, \mu) \cong\left(H^{\prime}, \mu^{\prime}\right)$ si y sólo si para cada $A \in \mathcal{A}$ existe un isomorfismo $\varsigma_{A}: H(A) \rightarrow H^{\prime}(A)$ tal que el siguiente diagrama:

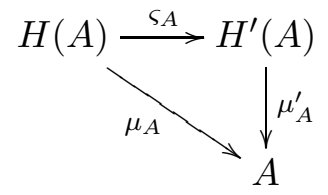

es conmutativo.

Lema 2.7. Sean $(H, \mu),\left(H^{\prime}, \mu^{\prime}\right) \in \operatorname{Pr}(\mathcal{A})$. Entonces, $(H, \mu) \leq\left(H^{\prime}, \mu^{\prime}\right) y$ $\left(H^{\prime}, \mu^{\prime}\right) \leq(H, \mu)$ si y sólo si $(H, \mu) \cong\left(H^{\prime}, \mu^{\prime}\right)$.

Demostración. Si $(H, \mu) \cong\left(H^{\prime}, \mu^{\prime}\right)$, entonces por la Definición 2.6, es claro que $(H, \mu) \leq\left(H^{\prime}, \mu^{\prime}\right)$ y $\left(H^{\prime}, \mu^{\prime}\right) \leq(H, \mu)$.

Ahora supongamos que $(H, \mu) \leq\left(H^{\prime}, \mu^{\prime}\right)$ y $\left(H^{\prime}, \mu^{\prime}\right) \leq(H, \mu)$ y probemos que $(H, \mu) \cong\left(H^{\prime}, \mu^{\prime}\right)$. Sea $A \in \mathcal{A}$. Ya que $(H, \mu) \leq\left(H^{\prime}, \mu^{\prime}\right)$, por la Definición 2.3, existe el siguiente diagrama conmutativo:

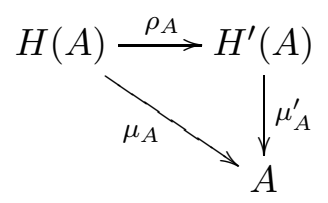

de donde, $\mu_{A}^{\prime} \circ \rho_{A}=\mu_{A}$. Por el hecho que $\left(H^{\prime}, \mu^{\prime}\right) \leq(H, \mu)$, del mismo modo que lo anterior, también existe un morfismo $\rho_{A}^{\prime}: H^{\prime}(A) \rightarrow H(A)$ tal que $\mu_{A} \circ \rho_{A}^{\prime}=$ $\mu_{A}^{\prime}$. Así:

$$
\begin{aligned}
\mu_{A} \circ \rho_{A}^{\prime} \circ \rho_{A} & =\mu_{A}^{\prime} \circ \rho_{A} \\
& =\mu_{A}=\mu_{A} \circ 1_{H(A)}
\end{aligned}
$$

donde $1_{H(A)}: H(A) \rightarrow H(A)$ es el morfismo identidad. Por ser $\mu_{A}$ un monomorfismo, de la ecuación (7), tenemos que $\rho_{A}^{\prime} \circ \rho_{A}=1_{H(A)}$. Análogamente a lo 
anterior, obtenemos que $\rho_{A}^{\prime} \circ \rho_{A}=1_{H^{\prime}(A)}$, donde $1_{H^{\prime}(A)}: H^{\prime}(A) \rightarrow H^{\prime}(A)$ es el morfismo identidad. Por lo tanto, $(H, \mu) \cong\left(H^{\prime}, \mu^{\prime}\right)$.

Lema 2.8. Sean $(H, \mu),\left(H^{\prime}, \mu^{\prime}\right) \in \operatorname{Pr}(\mathcal{A})$, tales que $(H, \mu) \cong\left(H^{\prime}, \mu^{\prime}\right)$. Entonces $\Phi(H, \mu) \cong \Phi\left(H^{\prime}, \mu^{\prime}\right)$, es decir, la asignación $\Phi$ preserva isomorfismos. Dualmente la asignación $\Psi$ preserva isomorfismos.

Demostración. Sean $(H, \mu),\left(H^{\prime}, \mu^{\prime}\right) \in \operatorname{Pr}(\mathcal{A})$, tales que $(H, \mu) \cong\left(H^{\prime}, \mu^{\prime}\right)$. Entonces, por el Lema 2.7, tenemos que $(H, \mu) \leq\left(H^{\prime}, \mu^{\prime}\right)$ y $\left(H^{\prime}, \mu^{\prime}\right) \leq(H, \mu)$. Por la Proposición 2.5, la asignación $\Phi$ preserva orden, así $\Phi(H, \mu) \leq \Phi\left(H^{\prime}, \mu^{\prime}\right)$ y $\Phi\left(H^{\prime}, \mu^{\prime}\right) \leq \Phi(H, \mu)$ en $\operatorname{Pr}(\mathcal{X})$. El resultado se concluye del Lema 2.7 , es decir, $\Phi(H, \mu) \cong \Phi\left(H^{\prime}, \mu^{\prime}\right)$.

La prueba de que $\Psi$ preserva isomorfismos es dual a la de $\Phi$.

Proposición 2.9. Sean $\mathcal{A}$ y $\mathcal{X}$ categorías equivalentes . Entonces

1. $\Psi \circ \Phi \cong 1_{\operatorname{Pr}(\mathcal{A})}$.

2. $\Phi \circ \Psi \cong 1_{\operatorname{Pr}(\mathcal{X})}$.

Donde la parte 1, significa que para $(H, \mu) \in \operatorname{Pr}(\mathcal{A}), \Psi(\Phi(H, \mu)) \cong(H, \mu)$. Análogamente para la parte 2.

Demostración. 1. Sea $(H, \mu) \in \operatorname{Pr}(\mathcal{A})$. Veamos que $\Psi(\Phi(H, \mu)) \cong(H, \mu)$. Por la Proposición 2.5:

$$
\Psi(\Phi(H, \mu))=\Psi(F H G, \varepsilon \circ F \mu G)=\left(G \circ F H G \circ F, \eta^{-1} \circ G v F\right),
$$

donde $v=\varepsilon \circ F \mu G$. Entonces probaremos que $\left(G \circ F H G \circ F, \eta^{-1} \circ G v F\right) \cong$ $(H, \mu)$. Sea $A \in \mathcal{A}$, por la Definición 2.6 veremos que existe un isomorfismo $\varsigma_{A}: H(A) \rightarrow(G \circ F H G \circ F)(A)$ tal que el siguiente diagrama:

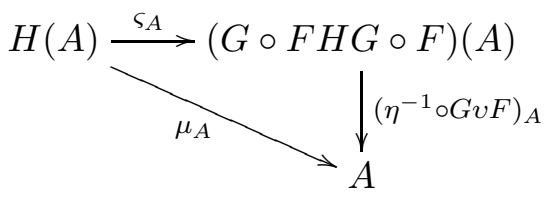

es conmutativo.

Tenemos el siguiente diagrama:

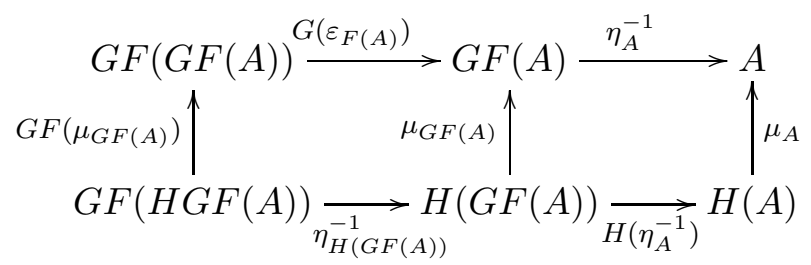

El cual es conmutativo por la naturalidad de $\eta$, puesto que $G\left(\varepsilon_{F(A)}\right)=\eta_{G F(A)}^{-1}$, y por ser $\mu$ una transformación natural.

Puesto que $\eta_{A}$ es un isomorfismo para toda $A \in \mathcal{A}$ y $H$ preserva isomorfismos, concluimos el resultado, es decir, en este caso $\varsigma_{A}^{-1}:=\eta_{H(G F(A))}^{-1} \circ H\left(\eta_{A}^{-1}\right)$. 
2. La prueba de que $\Phi \circ \Psi \cong 1_{\operatorname{Pr}(\mathcal{X})}$ es análoga a la anterior.

Como ya mencionamos al principio, nos interesa ver la relación entre las retículas $R$-pr y $S$-pr cuando las categorías de módulos $R$-Mod y $S$-Mod son equivalentes. Así, en lo que sigue, trabajaremos con la categoría $\mathcal{A}=R$-Mod.

Definición 2.10. Sea $(H, \mu) \in \operatorname{Pr}(R$-Mod $)$. Se define el prerradical $\sigma_{H}: R$-Mod $\rightarrow$ $R$-Mod tal que para cada $M \in R$-Mod, $\sigma_{H}(M):=\operatorname{Im}\left(\mu_{M}\right)$

Observación 2.11. Dado $(H, \mu) \in \operatorname{Pr}(R$-Mod $)$, para cada $M \in R$-Mod, se tiene el siguiente diagrama conmutativo:

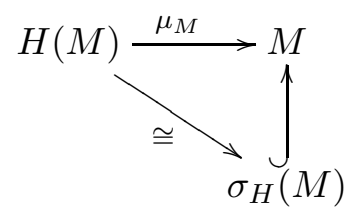

Puesto que $\mu_{M}$ es un monomorfismo, $H(M) \cong \sigma_{H}(M)$.

Por la Definición 2.10, la siguiente asignación está bien definida.

Proposición 2.12. Sea $(H, \mu) \in \operatorname{Pr}(R-M o d)$. La asignación $\pi_{R}: \operatorname{Pr}(R-M o d) \rightarrow$ $R$-pr definida como $\pi_{R}(H, \mu)=\sigma_{H}$, preserva orden.

Demostración. Sean $(H, \mu),\left(H^{\prime}, \mu^{\prime}\right) \in \operatorname{Pr}(R$-Mod $)$, tales que $(H, \mu) \leq\left(H^{\prime}, \mu^{\prime}\right)$ y veamos que $\sigma_{H} \preceq \sigma_{H^{\prime}}$. Sea $M \in R$-Mod, entonces se tiene un diagrama como en (4), donde $\rho_{M}: H(M) \rightarrow H^{\prime}(M)$ y así $\operatorname{Im} \mu_{M}=\operatorname{Im}\left(\mu_{M}^{\prime} \circ \rho_{M}\right) \leq \operatorname{Im}\left(\mu_{M}^{\prime}\right)$, es decir, $\sigma_{H}(M) \leq \sigma_{H^{\prime}}(M)$. Por lo tanto, $\sigma_{H} \preceq \sigma_{H^{\prime}}$, de donde concluimos que la asignación $\pi_{R}$ preserva orden.

Lema 2.13. Sean $(H, \mu),\left(H^{\prime}, \mu^{\prime}\right) \in \operatorname{Pr}(R$-Mod $)$. Entonces, $(H, \mu) \cong\left(H^{\prime}, \mu^{\prime}\right)$ si y solo si $\sigma_{H}=\sigma_{H^{\prime}}$.

Demostración. Sean $(H, \mu),\left(H^{\prime}, \mu^{\prime}\right) \in \operatorname{Pr}(R$-Mod $)$. Supongamos que $(H, \mu) \cong\left(H^{\prime}, \mu^{\prime}\right)$ y veamos que $\sigma_{H}=\sigma_{H^{\prime}}$. Sea $M \in R$-Mod, por la Definición 2.6, existe un isomorfismo $\varsigma_{M}: H(M) \rightarrow H^{\prime}(M)$ tal que el siguiente diagrama:

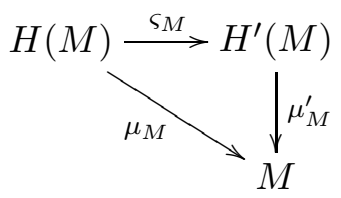

es conmutativo. De donde, $\operatorname{Im} \mu_{M}=\operatorname{Im}\left(\mu_{M}^{\prime} \circ \varsigma_{M}\right)=\operatorname{Im} \mu_{M}^{\prime}$, puesto que $\varsigma_{M}$ es isomorfismo. Por lo tanto $\sigma_{H}(M)=\sigma_{H^{\prime}}(M)$, de donde concluimos que $\sigma_{H}=$ $\sigma_{H^{\prime}}$.

Por otro lado, supongamos que $\sigma_{H}=\sigma_{H^{\prime}}$. Sea $M \in R$-Mod, por la Observación 2.11, tenemos que $H(M) \cong \sigma_{H}(M)=\sigma_{H^{\prime}}(M) \cong H^{\prime}(M)$. De ahí, se tiene 
un isomorfismo $\varsigma_{M}: H(M) \rightarrow H^{\prime}(M)$ y por lo tanto un diagrama conmutativo como (12). Por lo que, $(H, \mu) \cong\left(H^{\prime}, \mu^{\prime}\right)$.

El siguiente corolario, es inmediato de los Lemas 2.7 y 2.13.

Corolario 2.14. Sean $\left((H, \mu),\left(H^{\prime}, \mu^{\prime}\right) \in \operatorname{Pr}(R-M o d)\right)$. Las siguientes afirmaciones son equivalentes:

a) $(H, \mu) \leq\left(H^{\prime}, \mu^{\prime}\right) y\left(H^{\prime}, \mu^{\prime}\right) \leq(H, \mu)$.

b) $(H, \mu) \cong\left(H^{\prime}, \mu^{\prime}\right)$.

c) $\sigma_{H}=\sigma_{H^{\prime}}$

Ahora bien, dado $\sigma \in R$-pr, se tiene una inclusión natural $i_{\sigma}: \sigma \rightarrow 1_{R \text {-Mod }}$ definida para cada $M \in R$-Mod como $i_{\sigma M}: \sigma(M) \hookrightarrow M$ (inclusión de $\sigma(M)$ como submódulo de $M$ ) y que en particular es un monomorfismo natural.

Por lo tanto se tiene la siguiente asignación que está bien definida:

Lema 2.15. Sea $\sigma \in R$-pr. La asignación $\iota_{R}: R$-pr $\rightarrow \operatorname{Pr}(R-M o d)$ definida como $\iota_{R}(\sigma)=\left(\sigma, i_{\sigma}\right)$, preserva orden.

Demostración. El resultado es inmediato del orden en $R$-pr.

Lema 2.16. $\pi_{R} \circ \iota_{R}=1_{R-p r}$. Así, $\iota_{R}$ es inyectiva y $\pi_{R}$ es suprayectiva.

Demostración. Sea $\sigma \in R$-pr. Entonces $\pi_{R}\left(\iota_{R}(\sigma)\right)=\pi_{R}\left(\sigma, i_{\sigma}\right)=\tau_{\sigma}$, tal que para toda $M \in R$-Mod, $\tau_{\sigma}(M)=\operatorname{Im} i_{\sigma M}=\sigma(M)$, ver la Definición 2.10. Por lo tanto, $\pi_{R}\left(\iota_{R}(\sigma)\right)=\sigma$.

Lema 2.17. $\iota_{R} \circ \pi_{R} \cong 1_{\operatorname{Pr}(R-M o d)}$. Lo cual significa que dado $(H, \mu) \in \operatorname{Pr}(R$-Mod $)$, $\iota_{R}\left(\pi_{R}(H, \mu)\right) \cong(H, \mu)$.

Demostración. Sea $(H, \mu) \in \operatorname{Pr}(R$-Mod $)$. Entonces $\iota_{R}\left(\pi_{R}(H, \mu)\right)=\iota_{R}\left(\sigma_{H}\right)=$ $\left(\sigma_{H}, i_{\sigma_{H}}\right)$. Por lo que $\left(\sigma_{H}, i_{\sigma_{H}}\right) \cong(H, \mu)$, se sigue del Corolario 2.14.

Notemos que de los Lemas 2.16 y 2.17 se sigue que las estructuras ordenadas $\operatorname{Pr}(R$-Mod $)$ y $R$-pr consideradas como categorías son equivalentes para cualquier anillo $R$.

Recordamos de nuevo que queremos probar que hay un isomorfismo entre las retículas de prerradicales $R$-pr y $S$-pr cuando las categorías de módulos $R$-Mod y $S$-Mod son equivalentes, por lo que en la siguiente definición presentamos las asignaciones que nos servirán para dicho propósito.

Definición 2.18. Sean $R$ y $S$ anillos Morita-equivalentes. Definimos las siguientes asignaciones:

1. $\varphi:=\pi_{S} \circ \Phi \circ \iota_{R}: R-p r \rightarrow S-p r$.

2. $\psi:=\pi_{R} \circ \Psi \circ \iota_{S}: S-p r \rightarrow R-p r$. 
La siguiente observación, muestra de manera explícita cómo se definen las asignaciones $\varphi$ y $\psi$.

Observación 2.19. Sea $\tau \in R$-pr. Entonces la asignación $\varphi: R-p r \rightarrow S-p r$, está definida como:

$$
\begin{gathered}
R-p r \stackrel{\iota_{R}}{\longrightarrow} \operatorname{Pr}(R-M o d) \stackrel{\Phi}{\longrightarrow} \operatorname{Pr}(S-M o d) \stackrel{\pi_{S}}{\longrightarrow} S-p r \\
\tau \longmapsto\left(\tau, i_{\tau}\right) \longmapsto\left(F \tau G, \varepsilon \circ F i_{\tau} G\right) \longmapsto \sigma_{F \tau G}
\end{gathered}
$$

Así, $\varphi(\tau)=\sigma_{F \tau G}$.

Note que la descripción de la asignación $\varphi$, se puede ver como sigue:

Sean $\tau \in R$-pr y $N \in S$-Mod. Consideremos el siguiente diagrama:

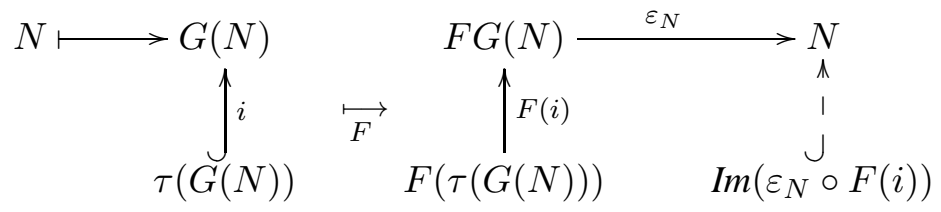

Entonces, $\varphi(\tau)(N):=\operatorname{Im}\left(\varepsilon_{N} \circ F(i)\right)$.

Por otro lado, sea $\sigma \in S$-pr. Entonces la asignación $\psi: S$-pr $\rightarrow R$-pr, está definida como:

$$
\begin{gathered}
S-p r \stackrel{\iota_{S}}{\longrightarrow} \operatorname{Pr}(S-M o d) \stackrel{\Psi}{\longrightarrow} \operatorname{Pr}(R-M o d) \stackrel{\pi_{R}}{\longrightarrow} S-p r \\
\sigma \longmapsto\left(\sigma, i_{\sigma}\right) \longmapsto\left(G \sigma F, \eta^{-1} \circ G i_{\sigma} F\right) \longmapsto \tau_{G \sigma F}
\end{gathered}
$$

donde, para cada $M \in R$-Mod, $\tau_{G \sigma F}(M)=\operatorname{Im}\left(\left(\eta^{-1} \circ G i_{\sigma} F\right)_{M}\right)$, ver la Definición 2.10. Así, $\psi(\sigma)=\tau_{G \sigma F}$.

La descripción de la asignación $\psi$, se puede ver como sigue:

Sean $\sigma \in S$-pr y $M \in R$-Mod. Consideremos el siguiente diagrama:
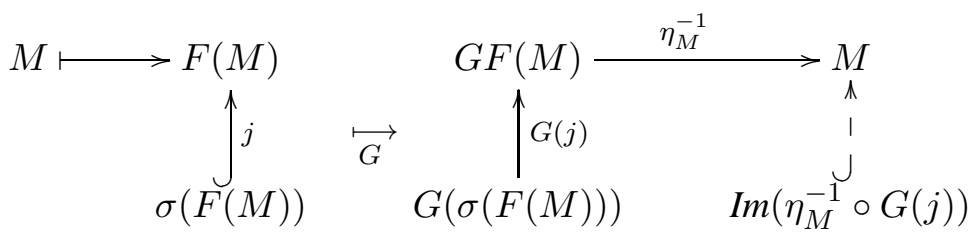

Entonces $\psi(\sigma)(M):=\operatorname{Im}\left(\eta_{M}^{-1} \circ G(j)\right)$. Obsérvese que para cada $M \in R$ Mod, podemos considerar $\eta_{M}^{-1}$, puesto que $\eta$ es un isomorfismo natural.

Proposición 2.20. Sean $R$ y $S$ anillos Morita-equivalentes. Las asignaciones $\varphi$ y $\psi$ preservan orden. 
Demostración. Se sigue del Lema 2.15 y de las Proposiciones 2.5 y 2.12.

Proposición 2.21. Sean $R$ y $S$ anillos Morita-equivalentes. Entonces $\psi \circ \varphi=1_{R-p r}$ $y \varphi \circ \psi=1_{S-p r}$.

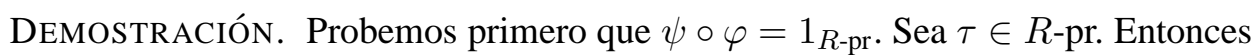
$\psi(\varphi(\tau))=\psi\left(\left(\pi_{S} \circ \Phi \circ \iota_{R}\right)(\tau)\right)=\psi\left(\left(\pi_{S} \circ \Phi\right)\left(\tau, i_{\tau}\right)\right)=\psi\left(\pi_{S}\left(F \tau G, \varepsilon \circ F i_{\tau} G\right)\right)$ $=\left(\pi_{R} \circ \Psi \circ \iota_{S}\right)\left(\pi_{S}\left(F \tau G, \varepsilon \circ F i_{\tau} G\right)\right)$

Por el Lema 2.17, $\left(\iota_{S} \circ \pi_{S}\right)\left(F \tau G, \varepsilon \circ F i_{\tau} G\right) \cong\left(F \tau G, \varepsilon \circ F i_{\tau} G\right)$. Luego, por el Lema 2.8 y 1 de la Proposición 2.9:

$$
\begin{aligned}
\Psi\left(\left(\iota_{S} \circ \pi_{S}\right)\left(F \tau G, \varepsilon \circ F i_{\tau} G\right)\right) & \cong \Psi\left(F \tau G, \varepsilon \circ F i_{\tau} G\right)=\Psi\left(\Phi\left(\tau, i_{\tau}\right)\right) \\
& \cong\left(\tau, i_{\tau}\right)
\end{aligned}
$$

De ahí, por el Corolario 2.14:

$$
\begin{aligned}
\pi_{R}\left(\Psi\left(\left(\iota_{S} \circ \pi_{S}\right)\left(F \tau G, \varepsilon \circ F i_{\tau} G\right)\right)\right) & =\pi_{R}\left(\tau, i_{\tau}\right)=\pi_{R}\left(\iota_{R}(\tau)\right) \\
& =\tau
\end{aligned}
$$

Por lo tanto $\psi(\varphi(\tau))=\tau$.

La prueba de que $\varphi \circ \psi=1_{S \text {-pr }}$, es dual a la anterior.

Ahora como consecuencia de la Proposición 1.10 (extendida a clases que no necesariamente son conjuntos) y de las Proposiciones 2.20 y 2.21, concluiremos que hay un isomorfismo entre las (grandes) retículas completas $R$-pr y $S$-pr.

Teorema 2.22. ( [3], [4] ) Sean $R$ y $S$ anillos Morita-equivalentes. Entonces las retículas $R$-pr y $S$-pr son isomorfas.

Ahora veamos que las asignaciones $\varphi$ y $\psi$ preservan los productos y coproductos de prerradicales. Primero trabajaremos en el caso de los productos de prerradicales, por lo que a continuación definimos el producto de dos miembros de la clase $\operatorname{Pr}(\mathcal{A})$, usando el producto estrella de transformaciones naturales.

Definición 2.23. Sean $(H, \mu),(K, \nu) \in \operatorname{Pr}(\mathcal{A})$. Se define el Producto en $\operatorname{Pr}(\mathcal{A})$, como $(K, \nu)(H, \mu):=(K H, \nu \circ K \mu)=(K H, \nu * \mu)$

Para ver que la asignación $\varphi$ preserva el producto de prerradicales mostraremos que cada una de las tres asignaciones que componen a $\varphi$ preservan los productos correspondientes.

Lema 2.24. La asignación $\iota_{R}$ preserva productos.

DEMOSTRACIÓN. Es clara.

Proposición 2.25. La asignación $\Phi: \operatorname{Pr}(\mathcal{A}) \rightarrow \operatorname{Pr}(\mathcal{X})$ preserva productos salvo isomorfismos. Esto es, dados $(H, \mu),(K, \nu) \in \operatorname{Pr}(\mathcal{A})$ se satisface que $\Phi((K, \nu)(H, \mu)) \cong \Phi(K, \nu) \Phi(H, \mu)$. 
Demostración. Sean $(H, \mu),(K, \nu) \in \operatorname{Pr}(\mathcal{A})$. Entonces, probemos que $\Phi((K, \nu)(H, \mu)) \cong \Phi(K, \nu) \Phi(H, \mu)$. Por las Definiciones 2.5 y 2.23:

$$
\Phi((K, \nu)(H, \mu))=\Phi(K H, \nu * \mu)=(F K H G, \varepsilon \circ F(\nu * \mu) G)
$$

$\mathrm{y}$

$$
\begin{aligned}
\Phi(K, \nu) \Phi(H, \mu) & =(F K G, \varepsilon \circ F \nu G)(F H G, \varepsilon \circ F \mu G) \\
& =(F K G F H G,(\varepsilon \circ F \nu G) *(\varepsilon \circ F \mu G)) \\
& =(F K G F H G,(\varepsilon * \varepsilon) \circ(F \nu G * F \mu G))
\end{aligned}
$$

Por lo tanto, veamos que

$$
(F K G F H G,(\varepsilon * \varepsilon) \circ(F \nu G * F \mu G)) \cong(F K H G, \varepsilon \circ F(\nu * \mu) G) .
$$

Para $A \in \mathcal{A}$ tenemos el siguiente diagrama conmutativo:

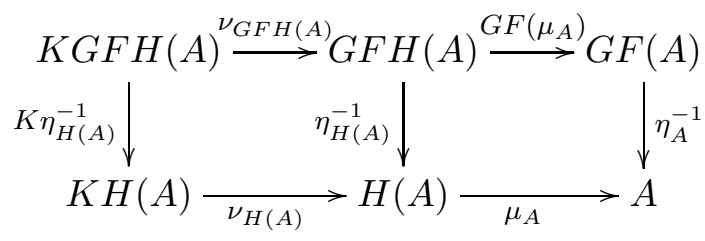

En donde, el diagrama de la izquierda conmuta por la naturalidad de $\nu$ y el de la derecha conmuta por la naturalidad de $\eta^{-1}$. Por lo tanto, el siguiente diagrama es conmutativo para toda $A \in \mathcal{A}$ :

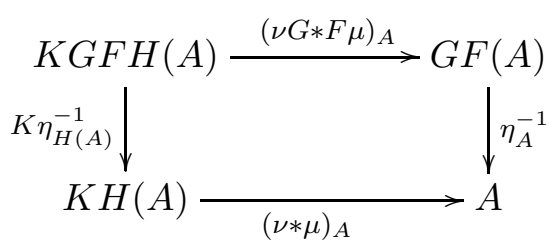

Sea $X \in \mathcal{X}$. Por la naturalidad de $\varepsilon$, tenemos el siguiente diagrama conmutativo:

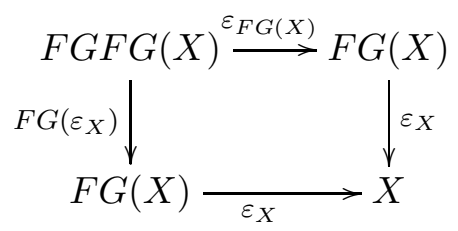

De los diagramas (15) y (16), obtenemos el siguiente diagrama conmutativo:

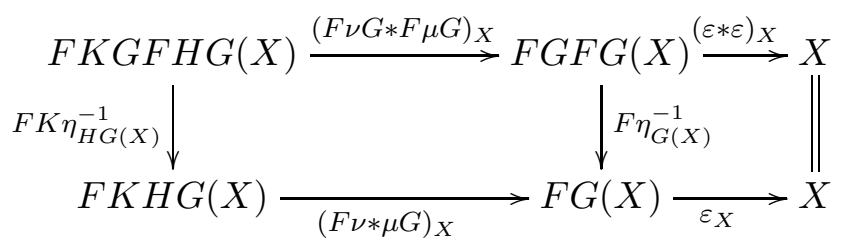

donde, $F \eta_{G(X)}^{-1}=\varepsilon_{F G(X)}$ y $F K \eta_{H G(X)}^{-1}$ son isomorfismos. Así por la Definición 2.6, se concluye que $\Phi((K, \nu)(H, \mu)) \cong \Phi(K, \nu) \Phi(H, \mu)$. 
Lema 2.26. La asignación $\pi_{R}$ preserva productos.

Demostración. Sean $(K, \nu),(H, \mu) \in \operatorname{Pr}(R$-Mod). Probemos que

$$
\pi_{R}((K, \nu)(H, \mu))=\pi_{R}(K, \nu) \pi_{R}(H, \mu) .
$$

Se tiene que $\pi_{R}((K, \nu)(H, \mu))=\pi_{R}(K H, \nu * \mu)=\sigma_{K H}$ y $\pi_{R}(K, \nu) \pi_{R}(H, \mu)=$ $\sigma_{K} \sigma_{H}$. Para $M \in R$-Mod, se tiene el siguiente diagrama conmutativo:

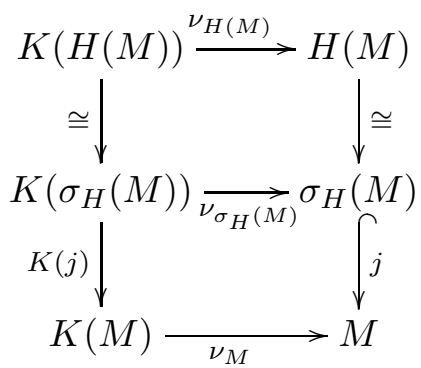

donde $j: \sigma_{H}(M) \hookrightarrow M$ es la inclusión natural y de ahí, $\operatorname{Im}(\nu * \mu)_{M}=\operatorname{Im} \nu_{\sigma_{H}(M)}$. Por lo tanto $\sigma_{K H}(M)=\sigma_{K}\left(\sigma_{H}(M)\right)$ y concluimos que $\pi_{R}((K, \nu)(H, \mu))=$ $\pi_{R}(K, \nu) \pi_{R}(H, \mu)$.

El siguiente corolario muestra que la asignación $\varphi$ preserva el producto de prerradicales.

Corolario 2.27. La asignación $\varphi$ preserva productos de prerradicales.

Demostración. Sean $\tau, \tau^{\prime} \in R$-pr. Veamos que $\varphi\left(\tau \tau^{\prime}\right)=\varphi(\tau) \varphi\left(\tau^{\prime}\right)$. Por el Lema 2.24:

$$
\varphi\left(\tau \tau^{\prime}\right)=\pi_{S} \Phi\left(\iota_{R}\left(\tau \tau^{\prime}\right)\right)=\pi_{S} \Phi\left(\iota_{R}(\tau) \iota_{R}\left(\tau^{\prime}\right)\right)=\pi_{S} \Phi\left(\left(\tau, i_{\tau}\right)\left(\tau^{\prime}, i_{\tau^{\prime}}\right)\right)
$$

Ahora por la Proposición 2.25, $\Phi\left(\left(\tau, i_{\tau}\right)\left(\tau^{\prime}, i_{\tau^{\prime}}\right)\right) \cong \Phi\left(\tau, i_{\tau}\right) \Phi\left(\tau^{\prime}, i_{\tau^{\prime}}\right)$. Por el Corolario 2.14 y el Lema 2.26:

$$
\begin{aligned}
\pi_{S} \Phi\left(\left(\tau, i_{\tau}\right)\left(\tau^{\prime}, i_{\tau^{\prime}}\right)\right) & =\pi_{S}\left(\Phi\left(\tau, i_{\tau}\right) \Phi\left(\tau^{\prime}, i_{\tau^{\prime}}\right)\right) \\
& =\pi_{S}\left(\Phi\left(\tau, i_{\tau}\right)\right) \pi_{S}\left(\Phi\left(\tau^{\prime}, i_{\tau^{\prime}}\right)\right)=\pi_{S} \Phi\left(\iota_{R}(\tau)\right) \pi_{S} \Phi\left(\iota_{R}\left(\tau^{\prime}\right)\right) \\
& =\varphi(\tau) \varphi\left(\tau^{\prime}\right)
\end{aligned}
$$

Por lo tanto $\varphi$ preserva el producto de prerradicales.

De la Proposición 2.21 y del Corolario 2.27, obtenemos que la asignación $\psi$ preserva el producto de prerradicales, como veremos enseguida.

Corolario 2.28. La asignación $\psi$ preserva productos de prerradicales.

Demostración. Sean $\sigma, \sigma^{\prime} \in S$-pr. Entonces probemos que $\psi\left(\sigma \sigma^{\prime}\right)=\psi(\sigma) \psi\left(\sigma^{\prime}\right)$. Sean $\tau:=\psi(\sigma)$ y $\tau^{\prime}:=\psi\left(\sigma^{\prime}\right)$. Entonces ya que $\varphi$ preserva productos y por la Proposición $2.21 \varphi$ y $\psi$ son inversas entre sí, se tiene que:

$$
\varphi\left(\tau \tau^{\prime}\right)=\varphi(\tau) \varphi\left(\tau^{\prime}\right)=\varphi(\psi(\sigma)) \varphi\left(\psi\left(\sigma^{\prime}\right)\right)=\sigma \sigma^{\prime} .
$$


De ahí:

$$
\psi\left(\sigma \sigma^{\prime}\right)=\psi\left(\varphi\left(\tau \tau^{\prime}\right)\right)=\tau \tau^{\prime}=\psi(\sigma) \psi\left(\sigma^{\prime}\right) .
$$

Por lo tanto $\psi$ preserva productos.

Para ver que las asignaciones $\varphi$ y $\psi$ preservan el coproducto de prerradicales se puede definir una clase $\operatorname{Pr}^{*}(\mathcal{A})$ dual a la clase $\operatorname{Pr}(\mathcal{A})$ cuyos miembros serán endofuntores y epimorfismos naturales. También sucede que en la clase $\operatorname{Pr}^{*}(\mathcal{A})$ el producto de dos de sus miembros se definirá usando de nuevo el producto estrella de transformaciones naturales, que en este caso son epimorfismos naturales.

En lo que resta de esta sección presentamos un desarrollo totalmente dual y análogo al que seguimos para ver que las asignaciones $\varphi$ y $\psi$ preservan el producto de prerradicales, para mostrar que dichas asignaciones preservan el coproducto de prerradicales.

Empecemos con las características de la clase $\operatorname{Pr}^{*}(\mathcal{A})$, que de nuevo vuelvo a recalcar, son duales a los de la clase $\operatorname{Pr}(\mathcal{A})$. Debido a esto, solamente escribiremos los resultados necesarios para obtener la preservación del coproducto de prerradicales bajo dichas asignaciones.

Definición 2.29. Sea $\mathcal{A}$ una categoría. Consideremos las siguientes parejas $(\boldsymbol{L}, \lambda)$, donde:

a) $\boldsymbol{L}: \mathcal{A} \rightarrow \mathcal{A}$ es un funtor $y$

b) $\lambda: 1_{\mathcal{A}} \rightarrow \boldsymbol{L}$ es un epimorfismo natural.

Se define la clase $\operatorname{Pr}^{*}(\mathcal{A})$ que consta de los miembros $(\boldsymbol{L}, \lambda)$.

Lema 2.30. Sea $(\boldsymbol{L}, \lambda) \in \operatorname{Pr}^{*}(\mathcal{A})$. Entonces $\boldsymbol{L}$ preserva epimorfismos.

El pre-orden $\leq$ en la clase $\operatorname{Pr}^{*}(\mathcal{A})$ es como sigue.

Definición 2.31. Sean $(\boldsymbol{L}, \lambda),(\boldsymbol{J}, \gamma) \in \operatorname{Pr}^{*}(\mathcal{A})$. Diremos que $(\boldsymbol{L}, \lambda) \leq(\boldsymbol{J}, \gamma)$ si $y$ sólo si para cada $A \in \mathcal{A}$ existe un triángulo conmutativo:

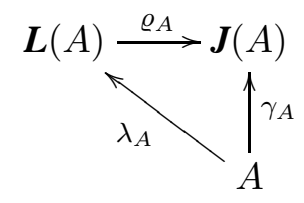

Proposición 2.32. Sean $\mathcal{X}$ y $\mathcal{A}$ categorías equivalentes. Las siguientes asignaciones están bien definidas y preservan orden:

a) $\Phi^{\prime}: \operatorname{Pr}^{*}(\mathcal{A}) \rightarrow \operatorname{Pr}^{*}(\mathcal{X})$ tal que para cada $(\boldsymbol{L}, \lambda) \in \operatorname{Pr}^{*}(\mathcal{A}), \Phi^{\prime}(\boldsymbol{L}, \mu)=$ $\left(F \boldsymbol{L} G, F \lambda G \circ \varepsilon^{-1}\right)$.

b) $\Psi^{\prime}: \operatorname{Pr}^{*}(\mathcal{X}) \rightarrow \operatorname{Pr}^{*}(\mathcal{A})$ tal que para cada $(\boldsymbol{J}, \gamma) \in \operatorname{Pr}^{*}(\mathcal{X}), \psi^{\prime}(\boldsymbol{J}, \gamma)=$ $(G \boldsymbol{J} F, G \gamma F \circ \eta)$. 
Para la siguiente proposición, necesitamos una definición dual a la Definición 2.6.

Proposición 2.33. Sean $\mathcal{A}$ y $\mathcal{X}$ categorías equivalentes . Entonces

1. $\Psi^{\prime} \circ \Phi^{\prime} \cong 1_{P r^{*}(\mathcal{A})}$.

2. $\Phi^{\prime} \circ \Psi^{\prime} \cong 1_{\operatorname{Pr}^{*}(\mathcal{X})}$.

Donde la parte 1 , significa que para $(\mathbf{L}, \lambda) \in \operatorname{Pr}^{*}(\mathcal{A}), \Psi^{\prime}\left(\Phi^{\prime}(\mathbf{L}, \lambda)\right) \cong(\mathbf{L}, \lambda)$. Análogamente para la parte 2 . $\operatorname{Pr}^{*}(\mathcal{A})$.

En lo que sigue definimos el producto de dos de los miembros de la clase

Definición 2.34. Sean $(\boldsymbol{L}, \lambda),(\boldsymbol{J}, \gamma) \in \operatorname{Pr}^{*}(\mathcal{A})$. Se define el Producto en $\operatorname{Pr}^{*}(\mathcal{A})$, como $(\boldsymbol{J}, \gamma)(\boldsymbol{L}, \lambda):=(\boldsymbol{J} \boldsymbol{L}, \gamma * \lambda)$.

El siguiente resultado es dual a la Proposición 2.25.

Proposición 2.35. Las asignaciones $\Phi^{\prime} y \Psi^{\prime}$ preservan productos salvo isomorfismos.

Definición 2.36. Sea $(\boldsymbol{L}, \lambda) \in \operatorname{Pr}^{*}(R$-Mod $)$. Se define el prerradical $\sigma^{\boldsymbol{L}}: R$-Mod $\rightarrow$ $R$-Mod tal que para cada $M \in R-M o d, \sigma^{\boldsymbol{L}}(M):=\operatorname{ker}\left(\lambda_{M}\right)$

Observación 2.37. Sea $(\boldsymbol{L}, \lambda) \in \operatorname{Pr}^{*}(R$-Mod $)$. Para cada $M \in R$-Mod, se tiene que $\lambda_{M}$ es un epimorfismo. Por lo que, $\boldsymbol{L}(M) \cong M / \sigma^{L}(M)$, dualmente a la Observación 2.11.

Proposición 2.38. Sea $(\boldsymbol{L}, \lambda) \in \operatorname{Pr}^{*}(R-M o d)$. La asignación $\pi_{R}^{*}: \operatorname{Pr}^{*}(R-M o d) \rightarrow$ $R$-pr definida como $\pi_{R}^{*}(\boldsymbol{L}, \lambda)=\sigma^{\boldsymbol{L}}$, preserva orden y manda productos en coproductos de prerradicales.

Ahora bien, para tener los resultados duales a los Lemas 2.15 y 2.24, trabajaremos con el funtor $\sigma^{*}$, el cual presentaremos en la siguiente definición, donde necesitaremos lo siguiente:

Sea $f: M \rightarrow N$ un morfismo en $R$-Mod y $\sigma \in R$-pr. Entonces se tienen las siguientes sucesiones exactas cortas y el diagrama conmutativo:

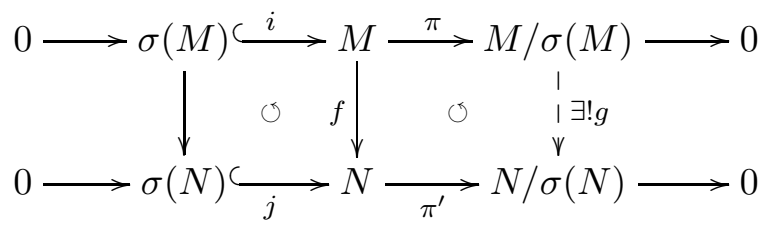

donde $i, j$ son las inclusiones canónicas y $\pi, \pi^{\prime}$ son las proyecciones canónicas. La existencia del $R$-homomorfismo $g: M / \sigma(M) \rightarrow N / \sigma(N)$ se sigue por el Teorema del Factor, puesto que $\pi^{\prime} \circ f \circ i=0$ ya que $f(\sigma(M)) \leq \sigma(N)$ por ser $\sigma$ un prerradical. 
Definición 2.39. Sea $\sigma \in R$-pr. Definimos el funtor $\sigma^{*}: R$-Mod $\rightarrow R$-Mod como:

1. $\sigma^{*}(M)=M / \sigma(M)$ para cada $M \in R$-Mod.

2. Si $f: M \rightarrow N$ es un homomorfismo en R-Mod, entonces con base en el párrafo anterior se define $\sigma^{*}(f)=g$.

Llamaremos al funtor $\sigma^{*}$ el coprerradical sobre $R$-Mod asociado a $\sigma$.

En lo que sigue, consideremos la transformación natural $p_{\sigma^{*}}: 1_{R \text {-Mod }} \rightarrow \sigma^{*}$.

Lema 2.40. Sea $\sigma \in R$-pr. La asignación $\iota_{R}^{*}: R$-pr $\rightarrow \operatorname{Pr}^{*}(R$-Mod $)$ definida como $\iota_{R}^{*}(\sigma)=\left(\sigma^{*}, p_{\sigma^{*}}\right)$, preserva orden y manda coproductos de prerradicales en productos.

Definición 2.41. Sean $R$ y $S$ anillos Morita-equivalentes. Definimos las siguientes asignaciones:

1. $\varphi^{*}:=\pi_{S}^{*} \circ \Phi^{\prime} \circ \iota_{R}^{*}: R-p r \rightarrow S-p r$.

2. $\psi^{*}:=\pi_{R}^{*} \circ \Psi^{\prime} \circ \iota_{S}^{*}: S-p r \rightarrow R-p r$.

Notemos que se tiene un resultado dual al que se tiene en el Corolario 2.14. De ahí y siguiendo las Definiciones 2.18 y 2.41, el lema a continuación no es difícil de verificar.

Lema 2.42. Las asignaciones $\varphi^{*} y \psi^{*}$ coinciden con $\varphi$ y $\psi$ respectivamente, donde estas últimas asignaciones son las de la Definición 2.18.

De manera similar al Corolario 2.27, se prueba el siguiente corolario.

Corolario 2.43. La asignación $\psi^{*}$ preserva coproductos de prerradicales.

Corolario 2.44. La asignación $\varphi^{*}$ preserva coproductos de prerradicales.

Demostración. Sean $\tau, \tau^{\prime} \in R$-pr. Entonces probemos que $\varphi^{*}\left(\tau: \tau^{\prime}\right)=$ $\left(\varphi^{*}(\tau): \varphi^{*}\left(\tau^{\prime}\right)\right)$. Sean $\sigma:=\varphi^{*}(\tau)$ y $\sigma^{\prime}:=\varphi^{*}\left(\tau^{\prime}\right)$. Luego de la Proposición 2.21 y del Lema 2.42 y puesto que $\psi^{*}$ preserva coproductos, se tiene que:

$$
\psi^{*}\left(\sigma: \sigma^{\prime}\right)=\left(\psi^{*}(\sigma): \psi^{*}\left(\sigma^{\prime}\right)\right)=\left(\psi^{*}\left(\varphi^{*}(\tau)\right): \psi^{*}\left(\varphi^{*}\left(\tau^{\prime}\right)\right)\right)=\left(\tau: \tau^{\prime}\right) .
$$

De ahí

$$
\varphi^{*}\left(\tau: \tau^{\prime}\right)=\varphi^{*}\left(\psi^{*}\left(\sigma: \sigma^{\prime}\right)\right)=\left(\sigma: \sigma^{\prime}\right)=\left(\varphi^{*}(\tau): \varphi^{*}\left(\tau^{\prime}\right)\right) .
$$

Por lo tanto $\varphi^{*}$ preserva coproductos.

Ya habíamos visto que las asignaciones $\varphi$ y $\psi$ preservan el producto de prerradicales, así que por el Lema 2.42 resumimos lo anterior en el siguiente corolario.

Corolario 2.45. Si $R$ y $S$ son anillos Morita-equivalentes, entonces existen isomorfismos de retículas completas que preservan productos y coproductos de prerradicales. 
Finalizamos esta sección mencionando que la prueba de la Proposición I.9.2 (5.2 de [3]) que enuncian Bican, Jambor, Kepka y Nẽmec en [4] y que en este trabajo dividimos en el Teorema 2.22 y el Corolario 2.45, se puede seguir directamente de los diagramas (13) y (14) que aparecen en la Observación 2.19, sin definir las clases $\operatorname{Pr}(\mathcal{A})$ y $\operatorname{Pr}^{*}(\mathcal{A})$. Esta técnica de demostración se desarrollará en la siguiente sección.

\subsection{Las retículas $R$-pr y $S$-pr cuando hay una situación de adjunción entre $R$-Mod y $S$-Mod}

En esta sección generalizaremos el Teorema 2.22 al caso en que se tiene una situación de adjunción entre las categorías $R$-Mod y $S$-Mod, es decir, sustituiremos la condición de que $R$ y $S$ son anillos Morita-equivalentes por dicha situación de adjunción, obteniendo una conexión de Galois entre las retículas $R$-pr y $S$-pr. Por lo que también daremos algunas propiedades de dicha conexión de Galois.

Teorema 2.46. Si F : R-Mod $\rightarrow S$-Mod es un funtor adjunto izquierdo de $G$ : $S$-Mod $\rightarrow R$-Mod. Entonces existe una conexión de Galois $\langle R-p r, \varphi, \psi, S$-pr $\rangle$ inducida por la adjunción $\langle F, G\rangle$.

Demostración. Puesto que hay una situación de adjunción entre las categorías $R$-Mod y $S$-Mod, existen funtores $F: R$-Mod $\rightarrow S$-Mod, $G: S$-Mod $\rightarrow R$-Mod y las transformaciones naturales $\eta: 1_{R \text {-Mod }} \rightarrow G F$ y $\varepsilon: F G \rightarrow 1_{S \text {-Mod }}$, tales que $(\varepsilon * F) \circ(F \circ \eta)=1_{F}$ y $(G * \varepsilon) \circ(\eta * G)=1_{G}$. La prueba consta de varias partes y primero definiremos las asignaciones $\varphi: R$-pr $\rightarrow S$-pr y $\psi: S$-pr $\rightarrow R$-pr

Descripción de las asignaciones $\varphi$ y $\psi$.

Sean $N \in S$-Mod y $\tau \in R$-pr. Consideremos el siguiente diagrama para describir $\varphi: R$-pr $\rightarrow S$-pr:

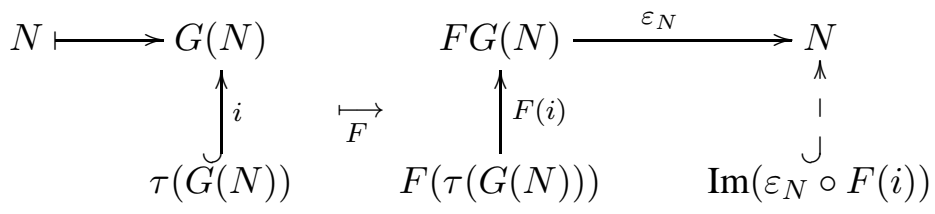

Entonces $\varphi(\tau)(N):=\operatorname{Im}\left(\varepsilon_{N} \circ F(i)\right)=\overrightarrow{\varepsilon_{N}}(\operatorname{Im} F(i))$, donde la última notación es la que aparece en (1) de la Sección 1.4 del Capítulo 1. Notemos que tal descripción es la misma que aparece en la Observación 2.19, usando el diagrama (13).

Por otro lado sean $M \in R$-Mod y $\sigma \in S$-pr. Consideremos el siguiente diagrama para describir $\psi: S$-pr $\rightarrow R$-pr:

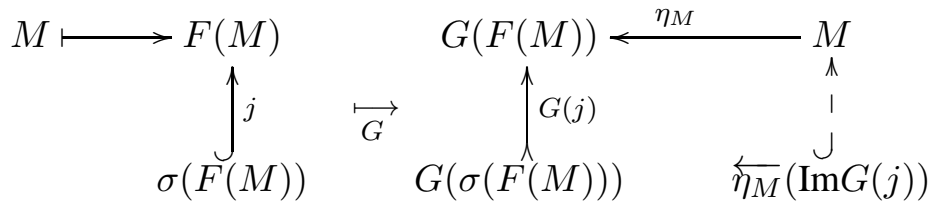


Entonces $\psi(\sigma)(M):=\overleftarrow{\eta_{M}}(\operatorname{Im}(G(j)))$. Notemos que esta descripción es diferente a la que se tiene usando el diagrama (14) que aparece en la Observación 2.19, puesto que en este caso la transformación natural $\eta: 1_{R \text {-Mod }} \rightarrow G F$ no necesariamente es un isomorfismo natural.

\section{$\varphi$ y $\psi$ están bien definidas.}

Sean $\sigma \in S$-pr y $M, M^{\prime} \in R$-Mod. Probemos que $\psi$ está bien definida, es decir, que $\psi(\sigma) \in R$-pr. Por la definición de $\psi$, es claro que $\psi(\sigma)(M) \leq M$. Sea $f: M \rightarrow M^{\prime}$ un $R$-homomorfismo, probaremos que $f(\psi(\sigma)(M)) \leq \psi(\sigma)\left(M^{\prime}\right)$. Consideremos el siguiente diagrama:

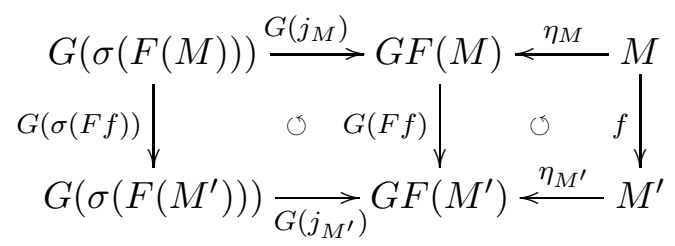

donde $j_{M}: \sigma(F(M)) \hookrightarrow F(M)$ y $j_{M^{\prime}}: \sigma\left(F\left(M^{\prime}\right)\right) \hookrightarrow F\left(M^{\prime}\right)$ son las inclusiones canónicas. El diagrama de la izquierda conmuta puesto que $\sigma \in S$-pr y puesto que el funtor $G$ preserva composiciones. El diagrama de la derecha conmuta por la naturalidad de $\eta$. De ahí obtenemos lo siguiente

$$
G(F f) \circ \eta_{M}=\eta_{M^{\prime}} \circ f .
$$

De donde:

$$
\overleftarrow{f}\left(\overleftarrow{\eta_{M^{\prime}}}\left(\operatorname{Im}\left(G\left(j_{M^{\prime}}\right)\right)\right)\right)=\overleftarrow{\eta_{M}}\left(\overleftarrow{G(F f)}\left(\operatorname{Im}\left(G\left(j_{M^{\prime}}\right)\right)\right)\right)
$$

Del diagrama anterior, vemos que $G(F f) \circ G\left(j_{M}\right)=G\left(j_{M^{\prime}}\right) \circ G(\sigma(F f))$. Entonces

$$
G(F f)\left(\operatorname{Im}\left(G\left(j_{M}\right)\right)\right)=\operatorname{Im}\left(G\left(j_{M^{\prime}}\right) \circ G(\sigma(F f))\right) \leq \operatorname{Im}\left(G\left(j_{M^{\prime}}\right)\right) .
$$

Por lo tanto $\operatorname{Im}\left(G\left(j_{M}\right)\right) \leq \overleftarrow{G(F f)}\left(\operatorname{Im}\left(G\left(j_{M^{\prime}}\right)\right)\right)$. Y de ahí

$$
\overleftarrow{\eta_{M}}\left(\operatorname{Im}\left(G\left(j_{M}\right)\right)\right) \leq \overleftarrow{\eta_{M}}\left(\overleftarrow{G(F f)}\left(\operatorname{Im}\left(G\left(j_{M^{\prime}}\right)\right)\right)\right)
$$

Por (20) y de las desigualdades anteriores:

$$
f\left(\overleftarrow{\eta_{M}}\left(\operatorname{Im}\left(G\left(j_{M}\right)\right)\right)\right) \leq f\left(\left(\overleftarrow{\eta_{M}}\left(\overleftarrow{G(F f)}\left(\operatorname{Im}\left(G\left(j_{M^{\prime}}\right)\right)\right)\right)\right) \leq \overleftarrow{\eta_{M^{\prime}}}\left(\operatorname{Im}\left(G\left(j_{M^{\prime}}\right)\right)\right)\right.
$$

es decir, $f(\psi(\sigma)(M)) \leq \psi(\sigma)\left(M^{\prime}\right)$. Por lo tanto $\psi(\sigma) \in R$-pr.

La prueba de que $\varphi$ está bien definida es análoga a la prueba anterior. Es decir, $\varphi(\tau) \in S$-pr, para $\tau \in R$-pr.

\section{$\varphi$ y $\psi$ preservan el orden.}

Veamos que $\psi$ preserva orden. Entonces, sean $\sigma, \sigma^{\prime} \in S$-pr, tal que $\sigma \preceq \sigma^{\prime}$ y sea $M \in R$-Mod. Consideremos las inclusiones canónicas $j_{\sigma}: \sigma(F(M)) \hookrightarrow$ $F(M), j_{\sigma^{\prime}}: \sigma^{\prime}(F(M)) \hookrightarrow F(M)$ e $i_{\sigma, \sigma^{\prime}}: \sigma(F(M)) \hookrightarrow \sigma^{\prime}(F(M))$ y el siguiente 
diagrama:

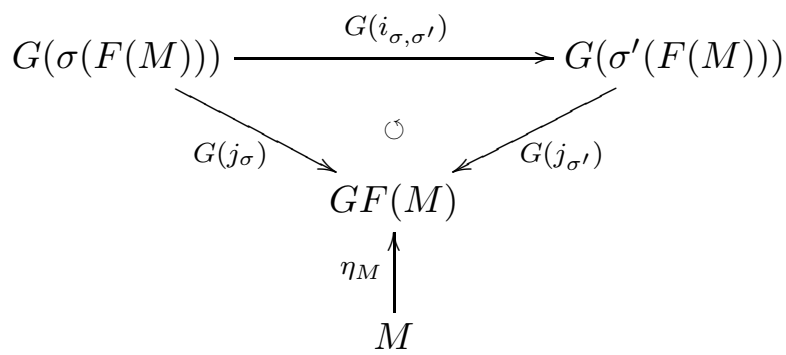

El diagrama (21) conmuta, puesto que $\sigma, \sigma^{\prime} \in S$-pr, $\sigma \preceq \sigma^{\prime}$ y el funtor $G$ preserva composiciones. De ahí

$$
\operatorname{Im}\left(G\left(j_{\sigma}\right)\right) \leq \operatorname{Im}\left(G\left(j_{\sigma^{\prime}}\right)\right) .
$$

Aplicando $\overleftarrow{\eta_{M}}$ obtenemos $\psi(\sigma)(M) \leq \psi\left(\sigma^{\prime}\right)(M)$. Por lo tanto $\psi(\sigma) \preceq \psi\left(\sigma^{\prime}\right)$, es decir, $\psi$ preserva orden.

La prueba de que $\varphi$ preserva el orden es análoga a la prueba de que $\psi$ preserva orden.

$\psi \circ \varphi$ es inflatoria.

Sea $\tau \in R$-pr y demostremos que $(\psi \circ \varphi)(\tau) \succeq \tau$. Sean $M \in R$-Mod y $j: \varphi(\tau)(F(M)) \hookrightarrow F(M)$ la inclusión canónica. Entonces $\psi(\varphi(\tau))(M)=$ $\overleftarrow{\eta_{M}}(\operatorname{Im}(G(j)))=\overleftarrow{\eta_{M}}(G(j)(G(\varphi(\tau)(F M))))$. Tenemos el siguiente diagrama conmutativo:

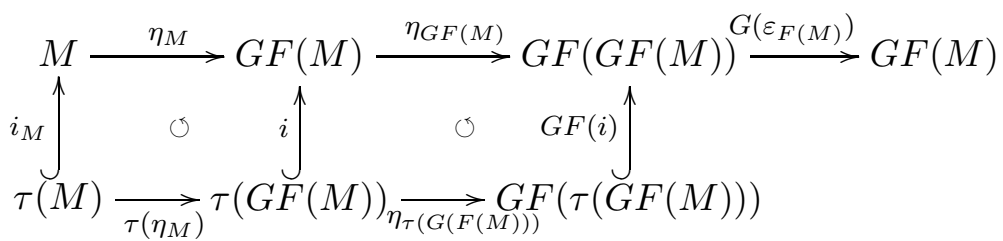

Puesto que $G\left(\varepsilon_{F(M)}\right) \circ \eta_{G F(M))}=1_{G F(M)}$, obtenemos

$$
\operatorname{Im}\left(\eta_{M} \circ i_{M}\right) \leq \operatorname{Im}\left(G\left(\varepsilon_{F(M)}\right) \circ G F(i)\right) .
$$

Ahora consideremos el siguiente diagrama:

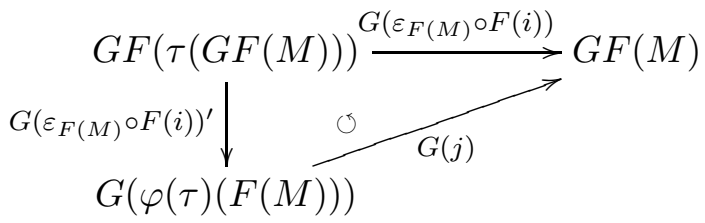

donde $\left(\varepsilon_{F(M)} \circ F(i)\right)^{\prime}$ es la correstricción del homomorfismo $\varepsilon_{F(M)} \circ F(i)$ a su imagen. De tal diagrama obtenemos

$$
\operatorname{Im}\left(G\left(\varepsilon_{F(M)} \circ F(i)\right)\right) \leq \operatorname{Im}(G(j)) .
$$

De (22) y (23) se deduce

$$
\overleftarrow{\eta_{M}}(\operatorname{Im}(G(j))) \geq \overleftarrow{\eta_{M}}\left(\operatorname{Im}\left(\eta_{M} \circ i_{M}\right)\right) \geq i_{M}(\tau(M))=\tau(M)
$$

Es decir, $\psi(\varphi(\tau))(M) \geq \tau(M)$. Por lo tanto $\psi \circ \varphi$ es inflatoria. 
$\varphi \circ \psi$ es deflatoria.

Sea $\sigma \in S$-pr y probemos que $(\varphi \circ \psi)(\sigma) \preceq \sigma$. Sean $N \in S$-Mod e $i$ : $\psi(\sigma)(G(N)) \hookrightarrow G(N)$ la inclusión canónica. Entonces

$$
\varphi(\psi(\sigma))(N)=\operatorname{Im}\left(\varepsilon_{N} \circ F(i)\right)=\left(\varepsilon_{N} \circ F(i)\right)(F(\psi(\sigma)(G(N)))) .
$$

Se tiene que $\psi(\sigma)(G(N))=\overleftarrow{\eta_{G(N)}}(\operatorname{Im} G(j))$, donde $\eta_{G(N)}: G(N) \rightarrow G F(G(N))$ y $j: \sigma(F G(N)) \hookrightarrow F G(N)$ es la inclusión canónica. Sea $B:=\operatorname{Im} G(j) \leq$ $G F(G(N))$ y denotemos por $h: \overleftarrow{\eta_{G(N)}}(B) \rightarrow B$ al homomorfismo inducido por $\eta_{G(N)}$. Consideremos el siguiente diagrama conmutativo, donde $i_{N}: \sigma(N) \hookrightarrow N$ e $i^{\prime}: B \hookrightarrow G F G(N)$ son las inclusiones naturales y $G(j)^{\prime}$ es el homomorfismo $G(j)$ correstringido a su imagen:

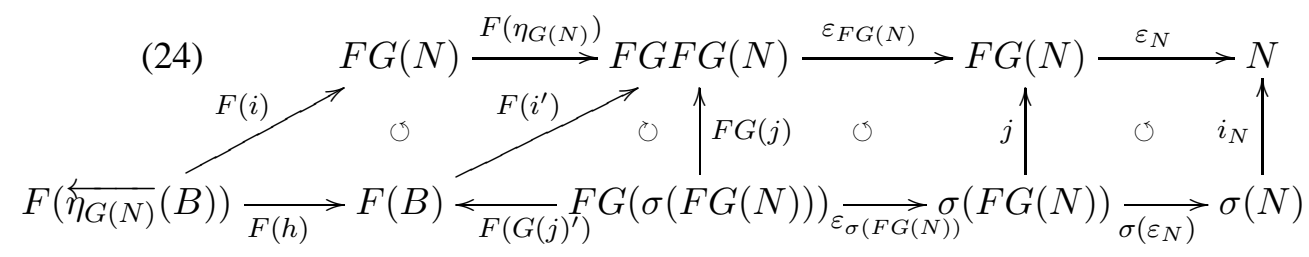

Puesto que $F$ preserva epimorfismos se tiene que $F\left(G(j)^{\prime}\right)$ es un epimorfismo y por tanto $\operatorname{Im}(F(h)) \subseteq \operatorname{Im}\left(F\left(G(j)^{\prime}\right)\right)$ y ya que $\varepsilon_{F G(N)} \circ F\left(\eta_{G(N)}\right)=1_{F G(N)}$, obtenemos

$$
\operatorname{Im}\left(\varepsilon_{N} \circ F(i)\right) \leq \operatorname{Im}\left(\varepsilon_{N} \circ \varepsilon_{F G(N)} \circ F G(j)\right) .
$$

De la desigualdad anterior y de los dos rectángulos de la derecha del diagrama (24) se concluye

$$
\varphi(\psi(\sigma))(N)=\operatorname{Im}\left(\varepsilon_{N} \circ F(i)\right) \leq \sigma(N) .
$$

Por lo tanto $\varphi \circ \psi$ es deflatoria.

De todo lo anterior y por la Proposición 1.17 (extendida a clases que no necesariamente son conjuntos), se concluye que $\langle R$-pr, $\varphi, \psi, S$-pr $\rangle$ es una conexión de Galois entre las (grandes) retículas completas $R$-pr y $S$-pr.

La siguiente observación muestra otra manera de cómo definir la asignación $\psi$ que aparece en la demostración del Teorema 2.46.

Observación 2.47. En la demostración del Teorema 2.46, la asignación $\psi: S$-pr $\rightarrow R$-pr se define para $\sigma \in S$-pr y $M \in R$-Mod, como $\psi(\sigma)(M):=$ $\overleftarrow{\eta_{M}}(\operatorname{Im}(G(j)))$, ver el Diagrama (19).

Observemos que $\psi$ se puede definir siguiendo un argumento análogo al que se hizo en la Definición 2.18 de la Sección 2.1, es decir, definiendo una asignación intermedia $\Psi: \operatorname{Pr}(S-M o d) \rightarrow \operatorname{Pr}(R-M o d)$, la cual veremos a continuación cómo se define.

Supongamos que hay una situación de adjunción entre las categorías $\mathcal{A}$ y $\mathcal{X}$. Por lo tanto existen los funtores $F: \mathcal{A} \rightarrow \mathcal{X}$ y $G: \mathcal{X} \rightarrow \mathcal{A}$ y las transformaciones naturales naturales $\eta: 1_{\mathcal{A}} \rightarrow G F$ y $\varepsilon: F G \rightarrow 1_{\mathcal{X}}$, tales que $(\varepsilon * F) \circ(F * \eta)=$ $1_{F},(G * \varepsilon) \circ(\eta * G)=1_{G}$. 
Supongamos que la categoría $\mathcal{X}$ tiene productos fibrados, ver la Sección 21 del Capítulo VI de [14]. Entonces, la asignación $\Psi: \operatorname{Pr}(\mathcal{X}) \rightarrow \operatorname{Pr}(\mathcal{A})$ aplicada a un objeto $(H, \mu) \in \operatorname{Pr}(\mathcal{X})$, se describe como $\Psi(H, \mu)$, el siguiente producto fibrado:

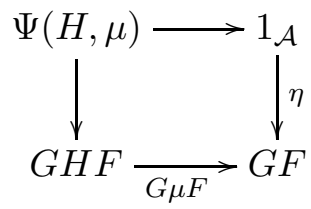

\subsection{Propiedades generales de la conexión de Galois $\langle R$-pr, $\varphi, \psi, S$-pr $\rangle$ inducida por la adjunción $\langle F, G\rangle$}

En esta sección estudiaremos algunas propiedades generales de la conexión de Galois $\langle R$-pr, $\varphi, \psi, S$-pr $\rangle$ inducida por la adjunción $\langle F, G\rangle$, donde $F: R$-Mod $\rightarrow$ $S$-Mod y $G: S$-Mod $\rightarrow R$-Mod.

Un problema interesante es describir a los prerradicales $\varphi\left(\alpha_{N}^{M}\right)$ y $\psi\left(\omega_{N}^{M}\right)$. Dado que los funtores $F$ y $G$ no necesariamente preservan inclusiones, es necesario generalizar la Definición 1.51 de los prerradicales alfa y omega de la siguiente manera:

Definición 2.48. Sean $N, M \in R$-Mod, $h: N \rightarrow M y k: M \rightarrow N$ homomorfismos en R-Mod. Se definen los siguientes prerradicales para todo $K \in R$-Mod:

1. $\alpha_{h}(K):=\sum\{\vec{f}(\operatorname{Im} h) \mid f: M \rightarrow K\}$.

2. $\omega_{k}(K):=\bigcap\{\overleftarrow{g}(\operatorname{ker} k) \mid g: K \rightarrow M\}$

Observación 2.49. Notemos que se dan las siguientes igualdades: $\alpha_{h}=\alpha_{\operatorname{Imh}}^{M} y$ $\omega_{k}=\omega_{\mathrm{ker} k}^{M}$.

Observación 2.50. Nótese que si $N \leq M$ e $i: N \hookrightarrow M$ es la inclusión natural y $p: M \rightarrow M / N$ es la proyección, entonces $\alpha_{i}=\alpha_{N}^{M} y \omega_{p}=\omega_{N}^{M}$.

El siguiente lema será importante para probar ciertos resultados acerca de cómo se comportan $\varphi$ y $\psi$ en los prerradicales $\alpha$ y $\omega$.

Lema 2.51. Sean $M, N, K, K^{\prime} \in R$-Mod. Supongamos que $K^{\prime} \leq K$ y que $h: N \rightarrow M$ es un R-homomorfismo. Si $H \subseteq \operatorname{Hom}_{R}(M, K)$, entonces $K^{\prime}=$ $\sum_{f \in H} \operatorname{Im} f h$, si y sólo si, existe un epimorfismo $\bar{g}: N^{(H)} \rightarrow K^{\prime}$ tal que para cada $f \in H$ el siguiente diagrama es conmutativo:

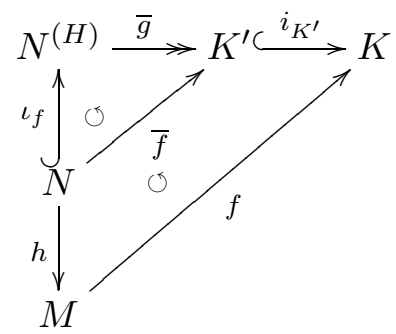


donde para cada $f \in H, \iota_{f}$ es la inclusión natural en el coproducto $N^{(H)}, \bar{f}$ es la correstricción del morfismo fh a su imagen e $i_{K^{\prime}}$ es la inclusión natural.

Demostración. Sea $K^{\prime}=\sum_{f \in H} \operatorname{Im} f h$. Entonces para cada $f \in H$, consideremos el morfismo $f h$, por la propiedad universal de la suma directa (Proposición 6.7, [2] ) existe un único $R$-homomorfismo $g: N^{(H)} \rightarrow K$ tal que el siguiente diagrama conmuta:

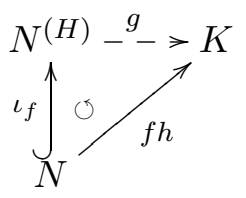

De ahí $\operatorname{Im} f h=\operatorname{Im}\left(g \circ \iota_{f}\right) \leq \operatorname{Im} g$. Pero se tiene que ( Proposición 6.8, [2])

$$
\operatorname{Im} g=g\left(N^{(H)}\right)=\sum_{f \in H} \operatorname{Im} f h=K^{\prime} .
$$

Por lo que $\operatorname{Im}(f h) \leq K^{\prime}$. Sea $\bar{f}$ la correstricción del morfismo $f h$ a su imagen. Entonces $\bar{f}: N \rightarrow K^{\prime}$ para cada $f \in H$. Luego, por la propiedad universal de la suma directa existe un único $R$-homomorfismo $\bar{g}: N^{(H)} \rightarrow K^{\prime}$ tal que el siguiente diagrama conmuta:

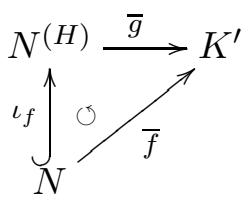

Puesto que

$$
\operatorname{Im} \bar{g}=\sum_{f \in H} \bar{f}(N)=\sum_{f \in H} f h(N)=K^{\prime},
$$

se obtiene que $\bar{g}$ es un epimorfismo. Es claro que $\left(i_{K^{\prime}}\right) \bar{f}=f h$ para cada $f \in H$. Por lo tanto el diagrama (25) es conmutativo.

Por otro lado supongamos que el diagrama (25) conmuta para cada $f \in H$ tal que $\bar{f}$ es la correstricción del morfismo $f h$ a su imagen. Entonces por la propiedad universal de la suma directa tenemos que

$$
K^{\prime}=\operatorname{Im} \bar{g}=\sum_{f \in H} \bar{f}(N)=\sum_{f \in H} \operatorname{Im} f h .
$$

Por lo tanto concluimos el resultado

Las siguientes proposiciones describen a los prerradicales $\varphi\left(\alpha_{h}\right)$ y $\psi\left(\omega_{h}\right)$.

Proposición 2.52. Sea $\langle R$-pr, $\varphi, \psi, S$-pr $\rangle$ la conexión de Galois inducida por la adjunción $\langle F, G\rangle$, donde $F: R$-Mod $\rightarrow S$-Mod y $G: S$-Mod $\rightarrow R$-Mod. Sean $N, M \in R$-Mod y $h: N \rightarrow M$ un homomorfismo en R-Mod. Entonces $\varphi\left(\alpha_{h}\right)=$ $\alpha_{F(h)}$. 
Demostración. Sea $L \in S$-Mod y consideremos el siguiente diagrama:

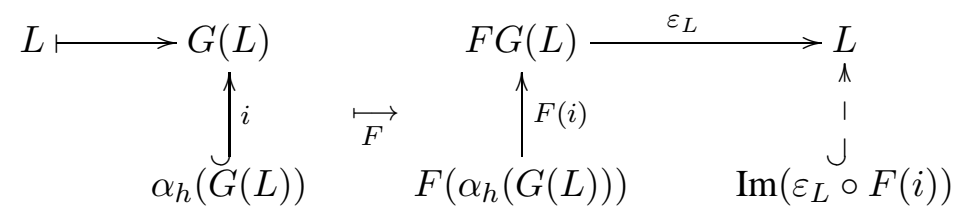

Entonces por definición, $\varphi\left(\alpha_{h}\right)(L):=\operatorname{Im}\left(\varepsilon_{L} \circ F(i)\right)=\left(\varepsilon_{L} \circ F(i)\right)\left(F\left(\alpha_{h}(G(L))\right)\right)$, donde

$$
\alpha_{h}(G(L))=\sum\left\{f(h(N)) \mid f \in \operatorname{Hom}_{R}(M, G(L))\right\} .
$$

Sea $H:=\operatorname{Hom}_{R}(M, G(L))$. Por el Lema 2.51, existe un epimorfismo $\bar{g}: N^{(H)} \rightarrow$ $\alpha_{h}(G(L))$ tal que el siguiente diagrama conmuta para cada $f \in H$ :

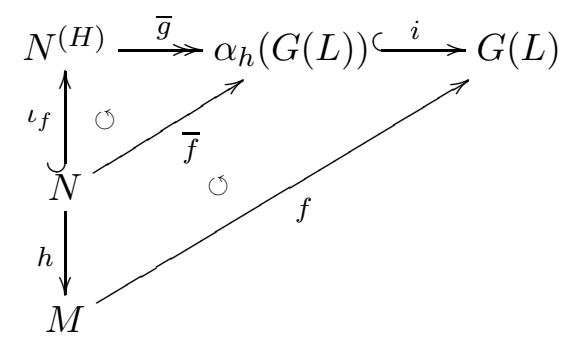

donde $\iota_{f}$ es la inclusión natural en el coproducto $N^{(H)}$ y $\bar{f}$ es la correstricción del morfismo $f h$ a su imagen. En virtud de las Proposiciones 1.40 y 1.42 , el funtor $F$ preserva epimorfismos y coproductos, por lo que aplicando el funtor $F$ al diagrama (26) obtenemos el siguiente diagrama conmutativo para cada $f \in H$ :

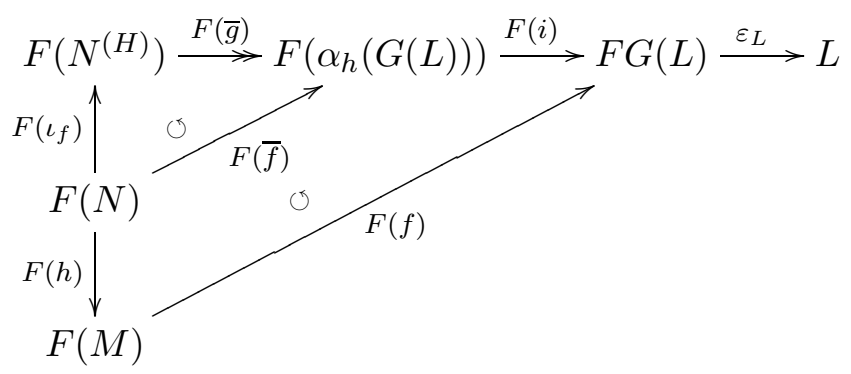

donde $F(\bar{g})$ es un epimorfismo y $F\left(N^{(H)}\right)$ es un coproducto. Por lo que del diagrama (27) y por la propiedad de la suma directa obtenemos:

$$
F\left(\alpha_{h}(G(L))\right)=\operatorname{Im} F(\bar{g})=\sum\{F(\bar{f})(F(N)) \mid f: M \rightarrow G(L)\},
$$

Por (27), $F(i) \circ F(\bar{f})=F(f) \circ F(h)$ y por (28) tenemos

$$
\begin{aligned}
F(i)\left(F\left(\alpha_{h}(G(L))\right)\right) & =\sum\{(F(i) \circ F(\bar{f}))(F(N)) \mid f: M \rightarrow G(L)\} \\
& =\sum\{(F(f) \circ F(h))(F(N)) \mid f: M \rightarrow G(L)\}
\end{aligned}
$$

Ya que $F$ es un funtor adjunto izquierdo de $G$, se tiene una biyección entre los conjuntos $\operatorname{Hom}_{S}(F(M), L)$ y $\operatorname{Hom}_{R}(M, G(L))$. Así que 


$$
\begin{aligned}
\left(\varepsilon_{L} \circ F(i)\right)\left(F\left(\alpha_{h}(G(L))\right)\right) & =\sum\left\{\left(\varepsilon_{L} \circ F(f) \circ F(h)\right)(F(N)) \mid f: M \rightarrow G(L)\right\} \\
& =\sum\{g(F(h)(F(N))) \mid g: F(M) \rightarrow L\} \\
& =\alpha_{F(h)}(L)
\end{aligned}
$$

Por lo tanto de (29), concluimos que $\varphi_{F}\left(\alpha_{h}\right)=\alpha_{F(h)}$.

Proposición 2.53. Sea $\langle R$-pr, $\varphi, \psi, S$-pr $\rangle$ la conexión de Galois inducida por la adjunción $\langle F, G\rangle$, donde $F: R$-Mod $\rightarrow S$-Mod y $G: S$-Mod $\rightarrow R$-Mod. Sean $N, M \in S$-Mod y $h: M \rightarrow N$ homomorfismos en $S$-Mod. Entonces $\psi\left(\omega_{h}\right)=$ $\omega_{G(h)}$.

Demostración. Sea $K \in R$-Mod y consideremos el siguiente diagrama
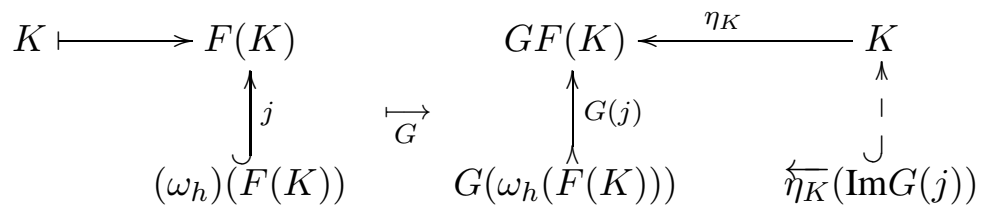

Entonces,por definición $\psi\left(\omega_{h}\right)(K):=\overleftarrow{\eta_{K}}(\operatorname{Im} G(j))$, donde

$$
\operatorname{Im} G(j)=G(j)\left(G\left(\omega_{h}(F(K))\right)\right)
$$

y

$$
\omega_{h}(F(K))=\bigcap\{\overleftarrow{g}(\operatorname{ker} h) \mid g: F(K) \rightarrow M\}
$$

Sea $H=\operatorname{Hom}_{S}(F(K), M)$ y $p_{h}: M \rightarrow M /$ kerh la proyección natural. Por la propiedad universal del producto directo existe un homomorfismo $l: F(K) \rightarrow$ $(M / \operatorname{ker} h)^{H}$, tal que para cada $g \in H$ el siguiente diagrama es conmutativo:

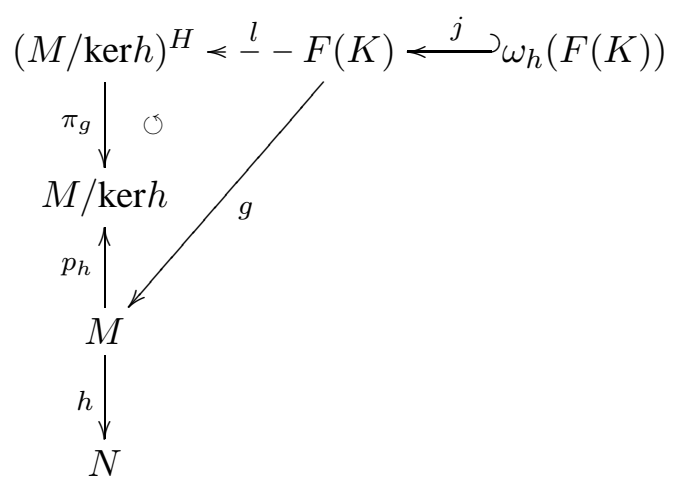


donde $\pi_{g}$ es la proyección natural del producto $(M / \operatorname{ker} h)^{H}$ y puesto que por la propiedad del producto directo se tiene:

$$
\begin{aligned}
\operatorname{ker} l & =\bigcap_{H} \operatorname{ker}\left(p_{h} \circ g\right)=\bigcap_{H} \overleftarrow{g}\left(\operatorname{ker} p_{h}\right)=\bigcap_{H} \overleftarrow{g}(\operatorname{ker} h) \\
& =\omega_{h}(F(K))
\end{aligned}
$$

Por las Proposiciones 1.40 y 1.42 el funtor $G$ preserva monomorfismos y productos, así que $G$ también preserva núcleos. Luego, aplicando el funtor $G$ al diagrama (30) obtenemos el siguiente diagrama conmutativo para cada $g \in H$

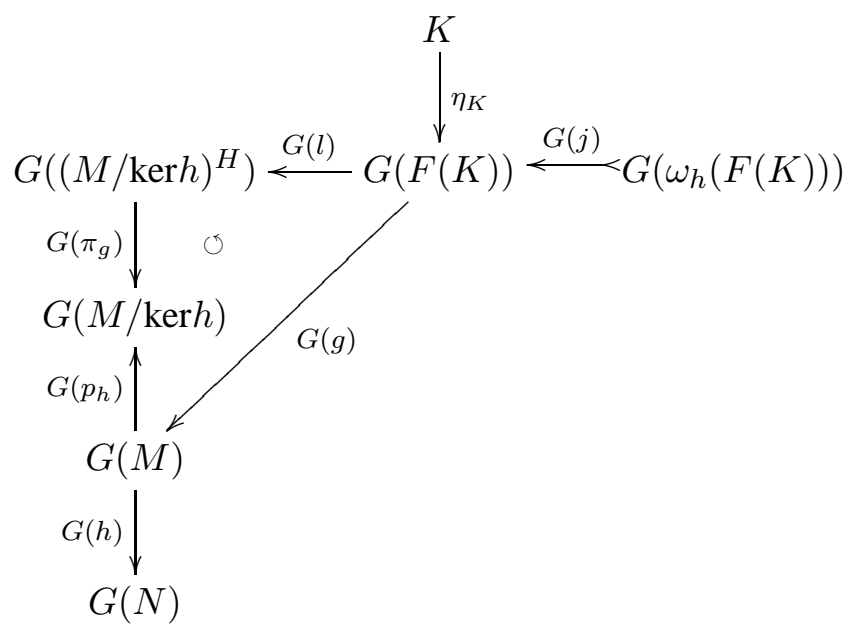

donde $G\left((M / \operatorname{ker} h)^{H}\right)$ es un producto, $\operatorname{ker} G\left(p_{h}\right)=\operatorname{ker} G(h)$ y $G(\operatorname{ker} l)=\operatorname{ker} G(l)$. Así que

$$
\begin{aligned}
\operatorname{Im} G(j) & =G(\operatorname{ker} l)=\operatorname{ker} G(l) \\
& =\bigcap\left\{\operatorname{ker}\left(G\left(p_{h}\right) \circ G(g)\right) \mid g: F(K) \rightarrow M\right\}
\end{aligned}
$$

Por ser $F$ un funtor adjunto izquierdo de $G$, usamos la biyección entre los conjuntos $\operatorname{Hom}_{S}(F(K), M)$ y $\operatorname{Hom}_{R}(K, G(M))$ y la igualdad anterior para obtener lo siguiente:

$$
\begin{aligned}
\psi\left(\omega_{h}\right)(K)=\overleftarrow{\eta_{K}}(\operatorname{Im} G(j)) & =\bigcap\left\{\overleftarrow{\eta_{K}}\left(\operatorname{ker}\left(G\left(p_{h}\right) \circ G(g)\right) \mid g: F(K) \rightarrow M\right\}\right. \\
& \bigcap\left\{\overleftarrow{\eta_{K}}\left(\overleftarrow{G(g)}\left(\operatorname{ker}\left(G\left(p_{h}\right)\right)\right) \mid g: F(K) \rightarrow M\right\}\right. \\
& \bigcap\left\{\overleftarrow{G(g) \circ \eta_{K}}\left(\operatorname{ker} G\left(p_{h}\right)\right) \mid g: F(K) \rightarrow M\right\} \\
& \bigcap\left\{\overleftarrow{G(g) \circ \eta_{K}}(\operatorname{ker} G(h)) \mid g: F(K) \rightarrow M\right\} \\
& \bigcap\{\overleftarrow{f}(\operatorname{ker} G(h)) \mid f: K \rightarrow G(M)\} \\
& =\omega_{G(h)}(K)
\end{aligned}
$$

Por lo tanto se concluye que $\psi\left(\omega_{h}\right)=\omega_{G(h)}$. 
En virtud del Teorema 1.58 todo prerradical se puede escribir en términos de los prerradicales alfa y omega. El siguiente resultado es consecuencia de las Proposiciones 2.52 y 2.53 y da una descripción alternativa de las asignaciones $\varphi$ y $\psi$. Esta descripción es importante porque ya no hace referencia a los diagramas que se usaron en la demostración del Teorema 2.46.

Teorema 2.54. Sean $\tau \in R$-pr y $\sigma \in S$-pr. Entonces

1. $\varphi(\tau)=\bigvee_{M \in R \text {-Mod }} \alpha_{F\left(i_{\tau M}\right)}$, donde $i_{\tau M}: \tau(M) \rightarrow M$ es la inclusión natural.

2. $\psi(\sigma)=\bigwedge_{N \in S-M o d} \omega_{G\left(p_{\sigma N}\right)}$, donde $p_{\sigma N}: N \rightarrow N / \sigma(N)$ es la proyección natural.

Demostración. Por el Teorema 1.58, $\tau=\bigvee_{M \in R \text {-Mod }} \alpha_{\tau(M)}^{M}=\bigvee_{M \in R \text {-Mod }} \alpha_{i_{\tau M}} \mathrm{y}$ puesto que $\varphi$ preserva supremos por la Proposición 1.16, tenemos

$$
\begin{aligned}
\varphi(\tau) & =\varphi\left(\bigvee_{M \in R-\operatorname{Mod}} \alpha_{i_{\tau M}}\right)=\bigvee_{M \in R \text {-Mod }} \varphi\left(\alpha_{i_{\tau M}}\right) \\
& =\bigvee_{M \in R \text {-Mod }} \alpha_{F\left(i_{\tau M}\right)}
\end{aligned}
$$

Análogamente por el Teorema $1.58, \sigma=\bigwedge_{N \in S-\text { Mod }} \omega_{\sigma(N)}^{N}=\bigwedge_{N \in S-\text { Mod }} \omega_{p_{\sigma N}} \mathrm{y}$ puesto que $\psi$ preserva ínfimos por la Proposición 1.16, tenemos

$$
\begin{aligned}
\psi(\sigma) & =\psi\left(\bigwedge_{N \in S-\operatorname{Mod}} \omega_{p_{\sigma N}}\right)=\bigwedge_{N \in S-\operatorname{Mod}} \psi\left(\omega_{p_{\sigma N}}\right) \\
& =\bigwedge_{N \in S-\operatorname{Mod}} \omega_{G\left(p_{\sigma N}\right)}
\end{aligned}
$$

Observación 2.55. Notemos que la descripción alternativa de $\varphi$ y que aparece en el Teorema 2.54, se puede usar en el caso cuando los anillos $R$ y $S$ son Moritaequivalentes, puesto que toda equivalencia de categorías da lugar a una adjunción.

Los morfismos $\varphi$ y $\psi$ preservan prerradicales idempotentes y radicales respectivamente como veremos en la siguiente proposición.

Proposición 2.56. Sea $\tau \in R$-pr y $\sigma \in S$-pr. Entonces

1. Para cada $M \in R-M o d, \varphi\left(\alpha_{M}^{M}\right)=\alpha_{F(M)}^{F(M)}$

2. Si $\tau$ es un prerradical idempotente, entonces $\varphi(\tau)$ es un prerradical idempotente en $S$-pr.

3. Para cada $N \in S$-Mod, $\psi\left(\omega_{0}^{N}\right)=\omega_{0}^{G(N)}$ 
4. Si $\sigma$ es radical, entonces $\psi(\sigma)$ es radical en $R$-pr.

Demostración. 1. Notemos que $\alpha_{M}^{M}=\alpha_{1_{M}}$ y aplicando $\varphi$, por la Proposición 2.52 se tiene el resultado.

2. Si $\tau \in R$-pr es un prerradical idempotente, entonces por la Proposición 1.69, $\tau=\bigvee_{M \in \mathbb{T}_{\tau}} \alpha_{M}^{M}$. Por lo tanto,

$$
\varphi(\tau)=\varphi\left(\bigvee_{M \in \mathbb{T}_{\tau}} \alpha_{M}^{M}\right)=\bigvee_{M \in \mathbb{T}_{\tau}} \varphi\left(\alpha_{M}^{M}\right)=\bigvee_{M \in \mathbb{T}_{\tau}} \alpha_{F(M)}^{F(M)}
$$

Es decir, $\varphi(\tau)$ es un prerradical idempotente en $S$-pr..

3. Notemos que $\omega_{0}^{N}=\omega_{1_{N}}$ y aplicando $\psi$, por la Proposición 2.53 se tiene el resultado.

4. Si $\sigma \in S$-pr es radical, entonces por la Proposición 1.69, $\sigma=\bigwedge_{N \in \mathbb{F}_{\sigma}} \omega_{0}^{N}$. Por lo tanto

$$
\psi(\sigma)=\psi\left(\bigwedge_{N \in \mathbb{F}_{\sigma}} \omega_{0}^{N}\right)=\bigwedge_{N \in \mathbb{F}_{\sigma}} \psi\left(\omega_{0}^{N}\right)=\bigwedge_{N \in \mathbb{F}_{\sigma}} \omega_{0}^{G(N)}
$$

Es decir, $\psi(\sigma)$ es radical.

Corolario 2.57. El prerradical $\varphi\left(1_{R}\right)$ es un prerradical idempotente y el prerradical $\psi\left(0_{S}\right)$ es radical.

Como consecuencia inmediata del hecho de que $\langle R$-pr, $\varphi, \psi, S$-pr $\rangle$ es una conexión de Galois cuando $F: R$-Mod $\rightarrow S$-Mod es un funtor adjunto izquierdo de $G: S$-Mod $\rightarrow R$-Mod, por el Corolario 1.18 se tiene que $\psi \circ \varphi$ es un operador cerradura en $R$-pr y $\varphi \circ \psi$ es un operador interior en $S$-pr. También por el Lema $1.19, \varphi\left(0_{R}\right)=0_{S}$ y $\psi\left(1_{S}\right)=1_{R}$, es decir, $0_{S}$ es un abierto de $S$-pr y $1_{R}$ es un cerrado de $R$-pr.

En el siguiente corolario, veremos ciertas propiedades de los prerradicales $\varphi\left(1_{R}\right)$ y $\psi\left(0_{S}\right)$ e introduciremos una notación para ellos. Como en lo anterior, $\varepsilon: F G \rightarrow 1_{S \text {-Mod }} \mathrm{y} \eta: 1_{R \text {-Mod }} \rightarrow G F$ son transformaciones naturales inducidas por la adjunción $\langle F, G\rangle$.

Corolario 2.58. Sean $\sigma_{\varepsilon}=\varphi\left(1_{R}\right) y \tau_{\eta}=\psi\left(0_{S}\right)$. Entonces

1. Para cada $N \in S$-Mod, $\sigma_{\varepsilon}(N)=\operatorname{Im}\left(\varepsilon_{N}\right)$

2. $\sigma_{\varepsilon}=\bigvee_{M \in R-M o d} \alpha_{F(M)}^{F(M)}$

3. $\sigma_{\varepsilon}=\varphi\left(1_{R}\right)$ es el mayor abierto en $S$-pr respecto al operador interior $\varphi \circ \psi$.

4. Para cada $M \in R-\operatorname{Mod}, \tau_{\eta}(M)=\operatorname{Ker}\left(\eta_{M}\right)$

5. $\tau_{\eta}=\bigwedge_{N \in S-M o d} \omega_{0}^{G(N)}$

6. $\tau_{\eta}=\psi\left(0_{S}\right)$ es el menor cerrado en $R$-pr respecto al operador cerradura $\psi \circ \varphi$. 
Demostración. 1. Sea $N \in S$-Mod. Entonces por la demostración del Teorema 2.46 y dado que en este caso $F(i): F G(N) \rightarrow F G(N)$, se tiene que $\varphi\left(1_{R}\right)(N)=$ $\operatorname{Im}\left(\varepsilon_{N} \circ F(i)\right)=\varepsilon_{N}(F G(N))=\operatorname{Im}\left(\varepsilon_{N}\right)$.

2. Se sigue del Teorema 2.54.

3. Por el Corolario 1.18, $\sigma_{\varepsilon}$ es un elemento abierto en $S$-pr. Sea $\sigma \in S$-pr un elemento abierto y puesto que $\psi(\sigma) \preceq 1_{R}$, obtenemos

$$
\sigma=\varphi(\psi(\sigma)) \preceq \varphi\left(1_{R}\right)=\sigma_{\varepsilon} .
$$

4. Sea $M \in R$-Mod. Entonces por la demostración del Teorema 2.46 y dado que en este caso $j: 0 \rightarrow F M$, se tiene que $\psi\left(0_{S}\right)(M)=\overleftarrow{\eta_{M}}(\operatorname{Im} G(j))=$ $\overleftarrow{\eta_{M}}(G(j) G(0))=\overleftarrow{\eta_{M}}(0)=\operatorname{Ker} \eta_{M}$

5. Se sigue del Teorema 2.54 .

6. Por el Corolario 1.18, $\tau_{\eta}$ es un elemento cerrado en $R$-pr. Sea $\tau \in R$-pr un elemento cerrado y ya que $\varphi(\tau) \succeq 0_{S}$, obtenemos

$$
\tau=\psi(\varphi(\tau)) \succeq \psi\left(0_{S}\right)=\tau_{\eta} .
$$

Observemos que cuando las categorías $R$-Mod y $S$-Mod son equivalentes, $\sigma_{\varepsilon}(N)=\operatorname{Im}\left(\varepsilon_{N}\right)=N$, puesto que en ese caso $\varepsilon_{N}$ es un isomorfismo. Es decir, en este caso $\varphi\left(1_{R}\right)=1_{S}$ y dualmente $\psi\left(0_{S}\right)=0_{R}$.

\subsection{Conexión de Galois inducida por el funtor tensor y el funtor Hom}

Para terminar este capítulo mostraremos una aplicación del Teorema 2.46, es decir, daremos algunos resultados de la conexión de Galois inducida por los funtores $F=U \bigotimes_{R}$ _ y $G=\operatorname{Hom}\left({ }_{S} U,{ }_{-}\right)$, donde $R$ y $S$ son anillos asociativos con 1 y ${ }_{S} U_{R}$ un bimódulo. A partir de los Teoremas 2.46 y 2.54 veremos dos descripciones para los morfismos $\varphi$ y $\psi$ de la conexión de Galois inducida por los funtores $F$ y $G$ y puesto que en [5] y [20] se caracaterizan a los pares adjuntos, dicha conexión de Galois más que una aplicación del Teorema 2.46 resulta ser un caso muy general.

Los siguientes funtores son con los que trabajaremos en esta sección y se pueden encontrar en [2]. El funtor que veremos en la primera definición ya lo habíamos mencionado en el Ejemplo 1.23 para una categoría $\mathcal{A}$ que en este caso será la categoría de $S$-módulos izquierdos, el codominio será la categoría de $R$-módulos izquierdos y consideraremos un bimódulo $U={ }_{S} U_{R}$. Se puede ver en la Sección 16 del Capítulo 5 de [2] cómo para cada $S$-módulo $M$, el conjunto $\operatorname{Hom}_{S}(U, M)$ es dotado de una estructura de $R$-módulo y también cómo para cada morfismo $f$ en $S$-Mod, $\operatorname{Hom}_{S}(U, f)$ es un $R$-homomorfismo.

Definición 2.59. Sean $R$ y $S$ anillos y $U={ }_{S} U_{R}$ un bimódulo. Entonces $\operatorname{Hom}_{S}\left(U, \_\right): S-M o d \rightarrow R$-Mod es un funtor definido como:

1. Para cada $M \in S-M o d, \operatorname{Hom}_{S}\left(U, \_\right)(M):=\operatorname{Hom}_{S}(U, M)$. 
2. Para cada homomorfismo $f: M \rightarrow N$ en $S$-Mod, $\operatorname{Hom}_{S}\left(U, \_\right)(f):=$ $\operatorname{Hom}_{S}(U, f)$, donde $\operatorname{Hom}_{S}(U, f): \operatorname{Hom}_{S}(U, M) \rightarrow \operatorname{Hom}_{S}(U, N)$ tal que para un S-homomorfismo $g: U \rightarrow M, \operatorname{Hom}_{S}(U, f)(g)=f \circ g$.

Definición 2.60. Sean $R$ y $S$ anillos y $U={ }_{S} U_{R}$ un bimódulo. Entonces $F=$ $U \bigotimes_{R}{ }_{-}: R$-Mod $\rightarrow S$-Mod es un funtor definido como:

1. Para cada $M \in R$-Mod, $F(M)=U \bigotimes_{R} M$.

2. Si $f: M \rightarrow N$ es un homomorfismo en $R$-Mod, se define $F(f):=$ $U \bigotimes_{R} f: U \bigotimes_{R} M \rightarrow U \bigotimes_{R} N$, tal que $\left(U \bigotimes_{R} f\right)(u \otimes m)=u \otimes f(m)$, donde $u \in U$ y $m \in M$.

El siguiente resultado es bien conocido, ver por ejemplo Capítulo IV de [19], y de acuerdo al Teorema 2.46 es el que nos llevará en esta sección a estudiar la conexión de Galois entre las retículas $R$-pr y $S$-pr.

Proposición 2.61. El funtor $F=U \bigotimes_{R}$ _ es un funtor adjunto izquierdo de $G=\operatorname{Hom}_{S}\left(U, \_\right)$

Por el Teorema 2.46 se tiene una conexión de Galois $\langle R$-pr, $\varphi, \psi, S$-pr $\rangle$ inducida por la adjunción $\langle F, G\rangle$, donde $\varphi: R$-pr $\rightarrow S$-pr y $\psi: S$-pr $\rightarrow R$-pr. En seguida veremos para este caso las descripciones de $\varphi$ y $\psi$, por lo que usaremos la notación $\langle\varphi, \psi\rangle$ para referirnos a esta conexión de Galois. Ver Observación 1.21.

Las siguientes proposiciones muestran dos prerradicales los cuales veremos que serán el resultado de evaluar los morfismos $\varphi$ y $\psi$.

Proposición 2.62. Sean $U={ }_{S} U_{R}$ un bimódulo y $\tau \in R$-pr. La $\tau$-traza respecto a $U$ se denota como ${ }_{\tau} \operatorname{Tr}_{U}\left(\_\right)$y se define para $N \in S$-Mod, como:

$$
{ }_{\tau} \operatorname{Tr}_{U}(N):=\sum\left\{\operatorname{Im} h \mid h \in \tau\left(\operatorname{Hom}_{S}(U, N)\right)\right\} .
$$

Entonces ${ }_{\tau} \operatorname{Tr}_{U}\left(\_\right)$es un prerradical sobre $S$.

Demostración. Sea $N \in S$-Mod. Para cada $h \in \tau(\operatorname{Hom}(U, N))$, se tiene que $h(U) \leq N$. Por lo tanto ${ }_{\tau} \operatorname{Tr}_{U}(N) \leq N$.

Ahora sea $f: N \rightarrow N^{\prime}$ un $S$-homomorfismo y veamos que:

$$
f\left({ }_{\tau} \operatorname{Tr}_{U}(N)\right) \leq{ }_{\tau} \operatorname{Tr}_{U}\left(N^{\prime}\right) .
$$

Sea $x \in f\left({ }_{\tau} \operatorname{Tr}_{U}(N)\right)$. Entonces $x=f(y)$, donde $y=\sum_{i=1}^{n} h_{i}\left(y_{i}\right)$, $h_{i} \in \tau\left(\operatorname{Hom}_{S}(U, N)\right)$ y $y_{i} \in U$ para $i=1, \ldots, n$. Puesto que $\tau \in R$-pr, se cumple que $\operatorname{Hom}_{S}(U, f)\left(\tau\left(\operatorname{Hom}_{S}(U, N)\right)\right) \leq \tau\left(\operatorname{Hom}_{S}\left(U, N^{\prime}\right)\right)$. Por lo que para cada $i=1, \ldots, n$, tenemos que $f \circ h_{i}=\operatorname{Hom}_{S}(U, f)\left(h_{i}\right) \in \tau\left(\operatorname{Hom}_{S}\left(U, N^{\prime}\right)\right)$.

Así, $f(y) \in{ }_{\tau} \operatorname{Tr}_{U}\left(N^{\prime}\right)$ puesto que $\left(f \circ h_{i}\right) \in \tau\left(\operatorname{Hom}_{S}\left(U, N^{\prime}\right)\right)$ y $\left(f \circ h_{i}\right)\left(y_{i}\right) \in$ $\left(f \circ h_{i}\right)(U)$. Por lo tanto, ${ }_{\tau} \operatorname{Tr}_{U} \in S$-pr. 
Ejemplo 2.63. Consideremos el prerradical $\tau=1_{R}$. En este caso la $\tau$-traza respecto a $U$ es ${ }_{\tau} \operatorname{Tr}_{U}\left(\_\right)=T r_{U}\left(\_\right)$, tal que para cada $N \in S$-Mod:

$$
\operatorname{Tr}_{U}(N)=\sum\left\{\operatorname{Im} h \mid h \in \operatorname{Hom}_{S}(U, N)\right\} .
$$

Es decir, la $\tau$-traza respecto a $U$ es la traza $\alpha_{U}^{U}$ respecto a $U$, ver Observación 1.52 .

Proposición 2.64. Sean $U={ }_{S} U_{R}$ un bimódulo y $\sigma \in S$-pr. El $\sigma$-anulador respecto a $U$ se denota como ${ }_{\sigma} A n n_{U}\left(\_\right)$y se define para $M \in R$-Mod, como:

$$
{ }_{\sigma} A_{n} n_{U}(M):=\left\{x \in M \mid u \otimes x \in \sigma\left(U \otimes_{R} M\right) \text { para cada } u \in U\right\},
$$

donde $u \otimes x$ es un generador de $U \bigotimes_{R} M$. Entonces ${ }_{\sigma} A n n_{U}\left(\_\right)$es un prerradical sobre $R$.

Demostración. Sea $M \in R$-Mod. Es claro que ${ }_{\sigma} \operatorname{Ann}_{U}(M) \leq M$. Sea $f: M \rightarrow M^{\prime}$ un morfismo en $R$-Mod y veamos que $f\left({ }_{\sigma} \operatorname{Ann}_{U}(M)\right) \leq$ ${ }_{\sigma} \operatorname{Ann}_{U}\left(M^{\prime}\right)$. Sea $x \in f\left({ }_{\sigma} \operatorname{Ann}_{U}(M)\right)$. Entonces $x=f(y)$, donde $y \in{ }_{\sigma} \operatorname{Ann}_{U}(M)$. Se tiene que $f(y) \in M^{\prime}$. Sea $u \in U$, probemos que $u \otimes f(y) \in \sigma\left(U \bigotimes_{R} M^{\prime}\right)$. Así

$$
u \otimes f(y)=\left(U \otimes_{R} f\right)(u \otimes y),
$$

donde $U \bigotimes_{R} f: U \bigotimes_{R} M \rightarrow U \bigotimes_{R} M^{\prime}$ es un morfismo en $S$-Mod. Puesto que $\sigma \in S$-pr, tenemos que $\left(U \bigotimes_{R} f\right)\left(\sigma\left(U \bigotimes_{R} M\right)\right) \leq \sigma\left(U \bigotimes_{R} M^{\prime}\right)$ y como $u \otimes y \in$ $\sigma\left(U \bigotimes_{R} M\right)$ para toda $u \in U$, de (32) obtenemos que $u \otimes f(y) \in \sigma\left(U \bigotimes_{R} M^{\prime}\right)$. Por lo tanto $\sigma \operatorname{Ann}_{U}\left(\_\right) \in R$-pr.

Ejemplo 2.65. Consideremos el prerradical $\sigma=0_{S}$. En este caso el $\sigma$-anulador respecto a $U$ es ${ }_{\sigma} A n n_{U}\left(\_\right)={ }_{0_{S}} A n n_{U}\left(\_\right)$, tal que para cada $M \in R$-Mod:

$$
0_{S} A n n_{U}(M)=\left\{x \in M \mid u \otimes x=0 \in U \otimes_{R} M \text { para cada } u \in U\right\}
$$

Es decir, el $\sigma$-anulador respecto a $U$ es el anulador respecto a $U$, tal como aperece en el Ejercicio 18 de la Sección 19 del Capítulo 5 de [2].

Observación 2.66. Puesto que el funtor $\operatorname{Hom}_{S}(U, \ldots)$ es exacto izquierdo entonces consideraremos a $\operatorname{Hom}_{S}(U, \sigma(N))$ un submódulo de $\operatorname{Hom}_{S}(U, N)$ para todo $\sigma \in$ $S$-pr y $N \in S$-Mod.

Proposición 2.67. Sea $\langle\varphi, \psi\rangle$ la conexión de Galois inducida por la adjunción $\langle F, G\rangle$. Entonces

1. Para cada $\tau \in R-p r, \varphi(\tau)={ }_{\tau} \operatorname{Tr}_{U}\left(\_\right)$.

2. Para cada $\sigma \in S-p r, \psi(\sigma)={ }_{\sigma} A n n_{U}\left(\_\right)$ 
Demostración. 1. Sean $\tau \in R$-pr y $N \in S$-Mod. Por la demostración del Teorema 2.46, el diagrama para describir $\varphi: R$-pr $\rightarrow S$-pr es el siguiente:

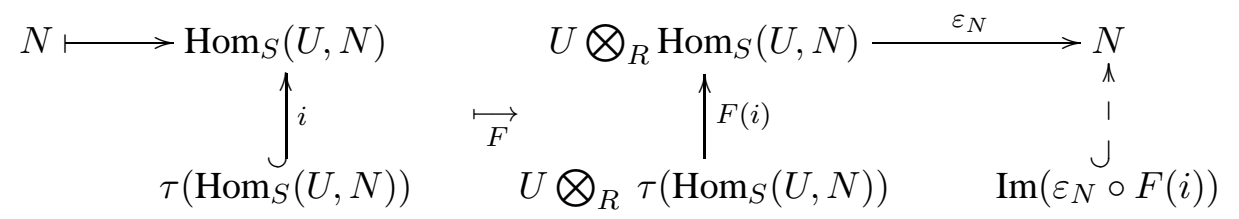

donde $\varepsilon_{N}$ es tal que $\varepsilon_{N}(u \otimes h)=h(u)$ para $u \in U$ y $h \in \tau\left(\operatorname{Hom}_{S}(U, N)\right)$. Entonces, por definición de $\varphi, \varphi(\tau)(N):=\operatorname{Im}\left(\varepsilon_{N} \circ F(i)\right)$, de donde obtenemos que $\varphi(\tau)={ }_{\tau} \operatorname{Tr}_{U}$.

2. Sea $\sigma \in S$-pr y $M \in R$-Mod. De nuevo por el Teorema 2.46, el diagrama para describir $\psi: S$-pr $\rightarrow R$-pr es el que sigue:

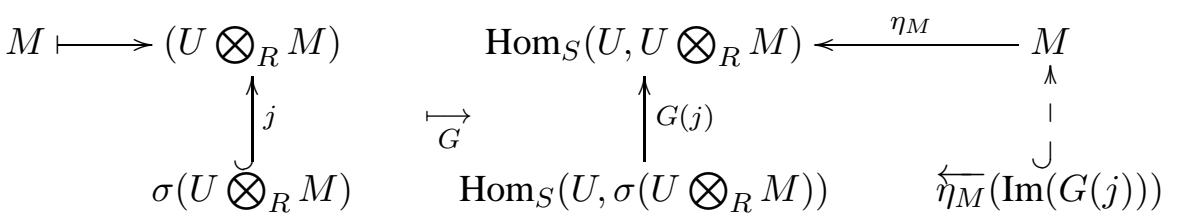

donde $\eta_{M}$ es tal que para cada $m \in M, \eta_{M}(m)=h_{m}$ y $h_{m}: U \rightarrow U \bigotimes_{R} M$ se define para cada $u \in U$ como $h_{m}(u)=u \otimes m$. Por definición de $\psi, \psi(\sigma)(M):=$ $\overleftarrow{\eta_{M}}(\operatorname{Im}(G(j)))$, de donde obtenemos que $\psi(\sigma)={ }_{\sigma} \operatorname{Ann}_{U}\left(\_\right)$.

Las descripciones de $\varphi$ y $\psi$ de la conexión de Galois inducida por la adjunción $\langle F, G\rangle$ las obtuvimos usando los diagramas del Teorema 2.46. El siguiente resultado es corolario del Teorema 2.54 y muestra la siguiente descripción para $\varphi$ y $\psi$.

Corolario 2.68. Sea $\langle\varphi, \psi\rangle$ la conexión de Galois inducida por la adjunción $\left\langle U \bigotimes_{R \_}, \operatorname{Hom}_{S}\left(U, \_\right)\right\rangle$. Entonces

1. Para cada $\tau \in R$-pr, $\varphi(\tau)=\bigvee_{M \in R \text {-Mod }} \alpha_{F\left(i_{\tau M}\right)\left(U \otimes_{R} \tau(M)\right)}^{U \otimes_{R} M}$, donde $i_{\tau M}$ : $\tau(M) \hookrightarrow M$ es la inclusión natural.

2. Para cada $\sigma \in S$-pr, $\psi(\sigma)=\bigwedge_{N \in S-M o d} \omega_{\operatorname{Hom}_{S}(U, \sigma(N))}^{\operatorname{Hom}_{(}(U)}$.

Demostración. 1. Sea $\tau \in R$-pr. Entonces por el Teorema 2.54:

$$
\varphi(\tau)=\bigvee_{M \in R-\operatorname{Mod}} \alpha_{F\left(i_{\tau M}\right)},
$$


donde $i_{\tau M}: \tau(M) \hookrightarrow M$ es la inclusión natural y $F\left(i_{\tau M}\right): U \bigotimes_{R} \tau(M) \rightarrow$ $U \bigotimes_{R} M$. Por lo que

$$
\begin{aligned}
\varphi(\tau) & =\bigvee_{M \in R-\operatorname{Mod}} \alpha_{\operatorname{Im} F\left(i_{\tau M}\right)}^{U \otimes_{R} M} \\
& =\bigvee_{M \in R \text {-Mod }} \alpha_{F\left(i_{\tau M}\right)\left(U \otimes_{R} \tau(M)\right)}^{U \otimes_{R} M}
\end{aligned}
$$

2. Sea $\sigma \in S$-pr. Entonces por el Teorema 2.54:

$$
\psi(\sigma)=\bigwedge_{N \in S \text {-Mod }} \omega_{G\left(p_{\sigma N}\right)},
$$

donde $p_{\sigma_{N}}: N \rightarrow N / \sigma(N)$ es la proyección canónica y $G\left(p_{\sigma N}\right): \operatorname{Hom}_{S}(U, N) \rightarrow$ $\operatorname{Hom}_{S}(U, N / \sigma(N))$. Por lo que

$$
\begin{aligned}
\psi(\sigma) & =\bigwedge_{N \in S \text {-Mod }} \omega_{\operatorname{ker} G\left(p_{\sigma N}\right)}^{G(N)} \\
& =\bigwedge_{N \in S \text {-Mod }} \omega_{\operatorname{Hom}_{S}(U, \sigma(N))}^{\operatorname{Hom}_{S}(U)}
\end{aligned}
$$

Corolario 2.69. Sean $\langle\varphi, \psi\rangle$ la conexión de Galois inducida por la adjunción $\left\langle U \otimes_{R}, H_{o m}\left(U, \_\right)\right\rangle, U={ }_{S} U_{R}$ un bimódulo, $M \in R$-Mod, $N \in S$-Mod, $\tau \in R$-pr y $\sigma \in S$-pr. Entonces

1. $\varphi(\tau)\left(U \bigotimes_{R} M\right) \geq F\left(i_{\tau M}\right)\left(U \bigotimes_{R} \tau(M)\right)$.

2. $\psi(\sigma)\left(\operatorname{Hom}_{S}(U, N)\right) \leq \operatorname{Hom}_{S}(U, \sigma(N))$.

Demostración. 1. Sea $\tau \in R$-pr. Entonces

$$
F\left(i_{\tau M}\right)\left(U \otimes_{R} \tau(M)\right) \leq \alpha_{F\left(i_{\tau M}\right)\left(U \otimes_{R} \tau(M)\right)}^{U \otimes_{R} M}\left(U \otimes_{R} M\right)
$$

Así del Corolario 2.68 concluimos que $\varphi(\tau)\left(U \bigotimes_{R} M\right) \geq F\left(i_{\tau M}\right)\left(U \bigotimes_{R} \tau(M)\right)$.

2. Sea $\sigma \in S$-pr. Entonces:

$$
\operatorname{Hom}_{S}(U, \sigma(N)) \geq \omega_{\operatorname{Hom}_{S}(U, \sigma(N))}^{\operatorname{Hom}_{(}(U, N)}\left(\operatorname{Hom}_{S}(U, N)\right) .
$$

Por lo tanto del Corolario 2.68, obtenemos que $\psi(\sigma)\left(\operatorname{Hom}_{S}(U, N)\right)$ $\leq \operatorname{Hom}_{S}(U, \sigma(N))$.

Cuando las categorías $R$-Mod y $S$-Mod son equivalentes, hemos visto en esta sección que las retículas $R$-pr y $S$-pr son isomorfas vía los morfismos $\varphi$ y $\psi$ los cuales preservan productos y coproductos de prerradicales. Cuando hay una situación de adjunción entre las categorías de módulos se tiene una desigualdad con respecto a los productos de prerradicales, como veremos adelante, así que el siguiente corolario nos acerca a dicho resultado.

Corolario 2.70. Sean $\langle\varphi, \psi\rangle$ la conexión de Galois inducida por la adjunción $\left\langle U \bigotimes_{R}, \operatorname{Hom}_{S}\left(U,{ }_{-}\right)\right\rangle, U={ }_{S} U_{R}$ un bimódulo, $M \in R$-Mod, $N \in S$-Mod, $\tau \in R$-pry $\sigma \in S$-pr. Entonces 
1. $\tau\left(\operatorname{Hom}_{S}(U, N)\right) \leq \operatorname{Hom}_{S}(U, \varphi(\tau)(N))$.

2. $F\left(i_{\psi(\sigma) M}\right)\left(U \bigotimes_{R} \psi(\sigma)(M)\right) \leq \sigma\left(U \bigotimes_{R} M\right)$, donde el morfismo $i_{\psi(\sigma) M}$ : $\psi(\sigma)(M) \hookrightarrow M$ es la inclusión canónica.

Demostración. 1. Sea $f \in \tau\left(\operatorname{Hom}_{S}(U, N)\right)$. Entonces $\operatorname{Im} f \leq{ }_{\tau} \operatorname{Tr}_{U}(N)$, así la correstricción $\bar{f}$ del morfismo $f$ a su imagen es tal que $\bar{f} \in \operatorname{Hom}_{S}(U, \varphi(\tau)(N))$. Por lo tanto $\tau\left(\operatorname{Hom}_{S}(U, N)\right) \leq \operatorname{Hom}_{S}(U, \varphi(\tau)(N))$.

2. Se tiene que $F\left(i_{\psi(\sigma) M}\right):=U \bigotimes_{R} i_{\psi(\sigma) M}: U \bigotimes_{R} \psi(\sigma)(M) \rightarrow U \bigotimes_{R} M$, donde $U \bigotimes_{R} i_{\psi(\sigma) M}(u \otimes y)=u \otimes y$, para $u \in U$ y $y \in \psi(\sigma)(M)$. Entonces $\operatorname{Im} F\left(i_{\psi(\sigma) M}\right)$ está generado por los elementos $u \otimes v$ donde $u \in U, v \in$ $\psi(\sigma)(M)={ }_{\sigma} \operatorname{Ann}_{U}(M)$ y $u \otimes v$ es un generador $U \otimes_{R} M$. Ahora como $v \in$ $\psi(\sigma)(M)={ }_{\sigma} \operatorname{Ann}_{U}(M)$, entonces $u \otimes v \in \sigma\left(U \otimes_{R} M\right)$ para toda $u \in U$, de donde obtenemos que $F\left(i_{\psi(\sigma) M}\right)\left(U \bigotimes_{R} \psi(\sigma)(M)\right) \leq \sigma\left(U \bigotimes_{R} M\right)$.

Note que los dos corolarios anteriores se pueden escribir de la siguiente manera:

Corolario 2.71. Sean $\langle\varphi, \psi\rangle$ la conexión de Galois inducida por la adjunción $\left\langle U \otimes_{R}, H_{S o m}\left(U, \_\right)\right\rangle, U={ }_{S} U_{R}$ un bimódulo, $\tau \in R$-pr, $\sigma \in S$-pr, $M \in R$ Mod y $N \in S$-Mod. Entonces

1. Para cada $\tau \in R-p r, \varphi(\tau)(F(M)) \geq F\left(i_{\tau(M)}\right)\left(U \bigotimes_{R} \tau(M)\right)$.

2. Para cada $N \in S$-Mod, $\tau(G(N))) \leq G(\varphi(\tau)(N))$.

3. Para cada $\sigma \in S$-pr, $\psi(\sigma)(G(N)) \leq G(\sigma(N))$.

4. Para cada $M \in R$-Mod, $F\left(i_{\psi(\sigma) M}\right)\left(U \bigotimes_{R} \psi(\sigma)(M)\right) \leq \sigma(F(M))$.

Los siguientes resultados muestran la desigualdad que se tiene con respecto al producto de prerradicales bajo los morfismos $\varphi$ y $\psi$.

Proposición 2.72. Sean $\langle\varphi, \psi\rangle$ la conexión de Galois inducida por la adjunción $\left\langle U \bigotimes_{R}, \operatorname{Hom}_{S}\left(U, \_\right)\right\rangle$y $U={ }_{S} U_{R}$ un bimódulo. Entonces $\varphi(\tau \sigma) \preceq \varphi(\tau) \varphi(\sigma)$ para cada $\tau, \sigma \in R$-pr.

Demostración. Sean $\tau, \sigma \in R$-pr y $N \in S$-Mod y veamos que $\varphi(\tau \sigma)(N) \leq$ $\varphi(\tau)(\varphi(\sigma)(N))$. Por 1 de la Proposición 2.67 :

$$
\begin{aligned}
\varphi(\tau \sigma)(N)= & { }_{\tau \sigma} \operatorname{Tr}_{U}(N) \\
& =\sum\left\{\operatorname{Im} h \mid h \in \tau \sigma\left(\operatorname{Hom}_{S}(U, N)\right)\right\} \\
& =\sum\left\{\operatorname{Im} h \mid h \in \tau\left(\sigma\left(\operatorname{Hom}_{S}(U, N)\right)\right)\right\}
\end{aligned}
$$

Por 1 del Corolario 2.70, tenemos que $\tau\left(\sigma\left(\operatorname{Hom}_{S}(U, N)\right)\right) \leq \tau\left(\operatorname{Hom}_{S}(U, \varphi(\sigma)(N))\right)$. Por lo tanto concluimos que $\varphi(\tau \sigma)(N) \leq \varphi(\tau)(\varphi(\sigma)(N))$, puesto que

$$
\varphi(\tau)(\varphi(\sigma)(N))=\sum\left\{\operatorname{Im} f \mid f \in \tau\left(\operatorname{Hom}_{S}(U, \varphi(\sigma)(N))\right)\right\} .
$$


Proposición 2.73. Sean $\langle\varphi, \psi\rangle$ la conexión de Galois inducida por la adjunción $\left\langle U \bigotimes_{R}, \operatorname{Hom}_{S}(U, \ldots)\right\rangle$ y $U={ }_{S} U_{R}$ un bimódulo. Entonces $\psi(\tau \sigma) \succeq \psi(\tau) \psi(\sigma)$ para cada $\tau, \sigma \in S$-pr.

Demostración. Sean $\sigma, \tau \in S$-pr y $M \in R$-Mod. Entonces, por 2 de la Proposición 2.67:

$$
\begin{aligned}
\psi(\tau)(\psi(\sigma)(M)) & ={ }_{\tau} \operatorname{Ann}_{U}(\psi(\sigma)(M)) \\
& =\left\{x \in \psi(\sigma)(M) \mid u \otimes x \in \tau\left(U \otimes_{R} \psi(\sigma)(M)\right) \text { para cada } u \in U\right\},
\end{aligned}
$$

donde $u \otimes x$ es un generador de $U \bigotimes_{R} \psi(\sigma)(M)$. También

$$
\begin{aligned}
\psi(\tau \sigma)(M) & ={ }_{\tau \sigma} \operatorname{Ann}_{U}(M) \\
& =\left\{x \in M \mid u \otimes x \in \tau \sigma\left(U \otimes_{R} M\right) \text { para cada } u \in U\right\},
\end{aligned}
$$

donde $u \otimes x$ es un generador de $U \bigotimes_{R} M$.

Sea $x \in \psi(\tau)(\psi(\sigma)(M))$ y probemos que $x \in \psi(\tau \sigma)(M))$. Sea $u \in U \mathrm{y}$ veamos que $u \otimes x \in \tau \sigma\left(U \bigotimes_{R} M\right)$, donde $u \otimes x$ un generador de $U \bigotimes_{R} M$. En efecto, $u \otimes x$ es un generador de $U \bigotimes_{R} M$, puesto que $x \in \psi(\sigma)(M) \leq M$.

Ahora, consideremos un generador en $U \bigotimes_{R} \psi(\sigma)(M)$, digamos $z=u \otimes x$, tal que $F\left(i_{\psi(\sigma) M}\right)(z)=u \otimes x$, donde $i_{\psi(\sigma) M}: \psi(\sigma)(M) \hookrightarrow M$ es la inclusión canónica y $F\left(i_{\psi(\sigma) M}\right): U \bigotimes_{R} \psi(\sigma)(M) \rightarrow U \bigotimes_{R} M$. De ahí, notemos que $u \otimes x \in F\left(i_{\psi(\sigma) M}\right)\left(U \bigotimes_{R} \psi(\sigma)(M)\right)$. Luego, puesto que $x \in \psi(\tau)(\psi(\sigma)(M))$, tenemos que $z=u \otimes x \in \tau\left(U \bigotimes_{R} \psi(\sigma)(M)\right)$. Por lo tanto, ya que $\tau \in R$-pr, obtenemos:

$$
\begin{aligned}
u \otimes x & =F\left(i_{\psi(\sigma) M}\right)(z) \in \tau\left(\operatorname{Im} F\left(i_{\psi(\sigma) M}\right)\right)=\tau\left(F\left(i_{\psi(\sigma) M}\right)\left(U \otimes_{R} \psi(\sigma)(M)\right)\right) \\
& \leq \tau\left(\sigma\left(U \otimes_{R} M\right)\right),
\end{aligned}
$$

donde la última desigualdad, se sigue de la parte 2 del Corolario 2.70. Así que $u \otimes x \in \tau \sigma\left(U \bigotimes_{R} M\right)$ para toda $u \in U$, donde $u \otimes x \in U \bigotimes_{R} M$.

El siguiente diagrama resume los argumentos de esta demostración:

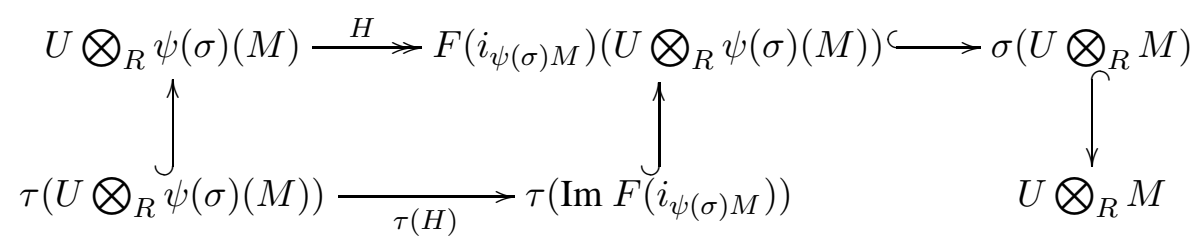

donde el morfismo $H$ es la correstricción del morfismo $F\left(i_{\psi(\sigma) M}\right)$ a su imagen. Concluimos entonces que $\psi(\tau) \psi(\sigma) \preceq \psi(\tau \sigma)$.

Observación 2.74. Cabe mencionar que la demostración de la Proposición 2.73, se puede obtener siguiendo la descripción de la asignación $\Psi$ que aparece en la Observación 2.47. Para esta técnica de demostración, se requiere la propiedad universal del producto fibrado, las características de la clase $\operatorname{Pr}(\mathcal{X})$, donde la categoría $\mathcal{X}$ tiene productos fibrados y por supuesto la Definición 2.23, en donde vemos cómo es el producto de dos de los miembros de la clase $\operatorname{Pr}(\mathcal{A})$. 
Los resultados que se obtienen en esta sección son muy importantes puesto que cada vez que se tiene una situación de adjunción entre las categorías $R$-Mod y $S$-Mod los funtores que relacionan a dichas categorías tienen una forma específica como afirma el Teorema de Watts-Eilenberg, que fue probado al mismo tiempo, ver [5] y [20]. Dicho resultado puede enunciarse de la siguiente manera:

Teorema 2.75. Sea $F: R$-Mod $\rightarrow S$-Mod un funtor adjunto izquierdo de $G:$ $S$-Mod $\rightarrow R$-Mod. Entonces existe un ${ }_{R} L_{S}$-bimódulo tal que $F \cong L \bigotimes_{R-y}$ $G \cong \operatorname{Hom}_{S}\left(L, \_\right)$

Con base en el Teorema anterior, los resultados que obtuvimos en esta sección son válidos para cualquier conexión de Galois entre $R$-pr y $S$-pr inducida por un par adjunto $\langle F, G\rangle$, es decir, los morfismos $\varphi$ y $\psi$ que relacionan a dichas retículas y evaluados en los prerradicales respectivos siempre tendrán la forma de $\tau$-traza y de $\sigma$-anulador respectivamente, con respecto al bimódulo $U$ que corresponde al par adjunto $\langle F, G\rangle$. Observemos también que en el Teorema 2.46 podemos incluir el comportamiento de los morfismos $\varphi$ y $\psi$ con respecto al producto de prerradicales, en el sentido de las Proposiciones 2.72 y 2.73 . 



\section{Capítulo 3}

\section{Morfismos entre retículas de prerradica- les asociados a transformaciones natura- les}

\section{1. $\varphi_{\gamma} \mathbf{y} \psi_{\gamma}$, con $\gamma$ una transformación natural general}

En la sección anterior dada una adjunción entre los funtores $F: R$-Mod $\rightarrow$ $S$-Mod y $G: S$-Mod $\rightarrow R$-Mod definimos morfismos entre las retículas de prerradicales $R$-pr y $S$-pr que son parte de la conexión de Galois entre dichas retículas. En esta sección aún no teniendo una adjunción entre categorías de módulos definiremos morfismos entre retículas de prerradicales que extienden bien los morfismos asociados a una adjunción, así como algunos resultados que generalizan a los de la sección anterior.

Una manera de conectar dos funtores es a través de una transformación natural, por lo que daremos morfismos entre las retículas $R$-pr y $S$-pr asociadas a una transformación natural.

En el Teorema 2.54 para evaluar $\varphi$ en un $R$-prerradical $\tau$ usamos al prerradical alfa generalizado en el funtor que va de $R$-pr a $S$-pr aplicado a la inclusión $i_{\tau M}$ : $\tau(M) \hookrightarrow M$, con $M \in R$-Mod. Para evaluar $\psi$ en un $S$-prerradical $\sigma$ usamos el prerradical generalizado omega en el funtor de regreso aplicado a la proyección $p_{\sigma N}: N \rightarrow N / \sigma(N)$ con $N \in S$-Mod.

Con base en lo anterior y usando los siguientes diagramas conmutativos definiremos morfismos entre retículas de prerradicales asociadas a una transformación natural. Sea $\gamma: F \rightarrow G$ una transformación natural entre los funtores $F, G$ : $R$-Mod $\rightarrow S$-Mod. Para la inclusión $i_{\tau M}: \tau(M) \hookrightarrow M$ donde $M \in R$-Mod, se tiene el siguiente diagrama conmutativo:

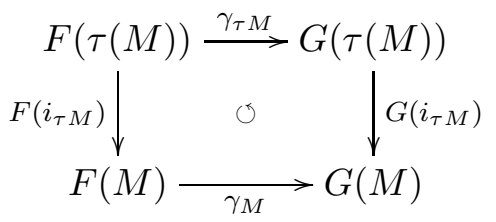


Para la proyección $p_{\tau M}: M \rightarrow M / \tau(M)$ con $M \in R$-Mod, tenemos el siguiente diagrama conmutativo:

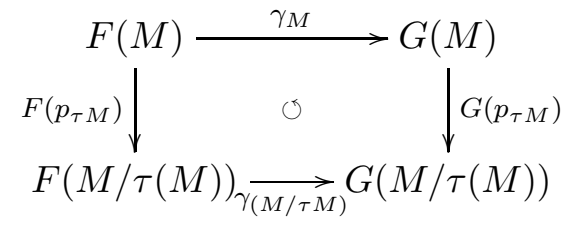

La siguiente definición es una generalización de la definición que se muestra en el Teorema 2.54.

Definición 3.1. Sean F, G:R-Mod $\rightarrow$ S-Mod funtores y $\gamma: F \rightarrow G$ una transformación natural. Se definen las siguientes asignaciones, para $\tau \in R$-pr como:

1. $\varphi_{\gamma}: R$-pr $\rightarrow$ S-pr como $\varphi_{\gamma}(\tau)=\bigvee_{M \in R \text {-Mod }} \alpha_{\gamma_{M} \circ F\left(i_{\tau M}\right)}$, donde $i_{\tau M}:$ $\tau(M) \hookrightarrow M$ es la inclusión natural.

2. $\psi_{\gamma}: R$-pr $\rightarrow$ S-pr como $\psi_{\gamma}(\tau)=\bigwedge_{M \in R-M o d} \omega_{\gamma_{(M / \tau M)} \circ F\left(p_{\tau M}\right)}$, donde $p_{\tau M}: M \rightarrow M / \tau(M)$ es la proyección natural.

Teorema 3.2. Las asignaciones $\varphi_{\gamma}$ y $\psi_{\gamma}$ son morfismos de clapos.

Demostración. Es claro que las asignaciones $\varphi_{\gamma}$ y $\psi_{\gamma}$ están bien definidas.

Veamos que $\varphi_{\gamma}$ preserva el orden. Sean $\tau, \tau^{\prime} \in R$-pr tal que $\tau \preceq \tau^{\prime}$ y probemos que $\varphi_{\gamma}(\tau) \preceq \varphi_{\gamma}\left(\tau^{\prime}\right)$. Basta demostrar que para toda $M \in R$-Mod, $\alpha_{\gamma_{M} \circ F\left(i_{\tau M}\right)} \preceq$ $\alpha_{\gamma_{M} \circ F\left(i_{\tau^{\prime} M}\right)}$, donde $i_{\tau^{\prime} M}: \tau^{\prime}(M) \hookrightarrow M$ es la inclusión canónica. Sean $M \in R$ Mod y $K \in S$-Mod. Entonces, por la Definición 2.48:

$$
\alpha_{\gamma_{M} \circ F\left(i_{\tau M}\right)}(K)=\sum\left\{f\left(\operatorname{Im}\left(\gamma_{M} \circ F\left(i_{\tau M}\right)\right)\right) \mid f: G(M) \rightarrow K\right\}
$$

$\mathrm{y}$

$$
\alpha_{\gamma_{M} \circ F\left(i_{\tau^{\prime} M}\right)}(K)=\sum\left\{g\left(\operatorname{Im}\left(\gamma_{M} \circ F\left(i_{\tau^{\prime} M}\right)\right)\right) \mid g: G(M) \rightarrow K\right\} .
$$

Se tiene el siguiente diagrama conmutativo:

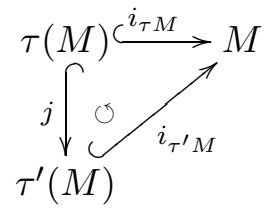

donde $j$ es la inclusión natural. Sea $f: G(M) \rightarrow K$. Entonces aplicando el funtor $\mathrm{F}$ al diagrama (34), obtenemos el siguiente diagrama conmutativo:

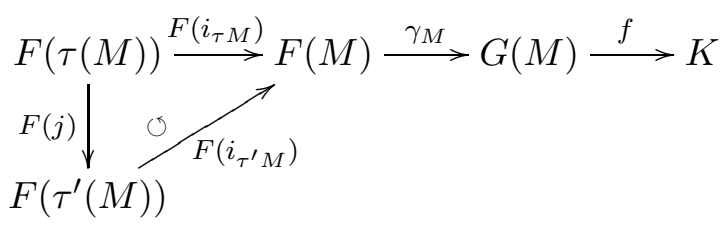


De donde obtenemos:

$$
\begin{aligned}
\operatorname{Im}\left(f \circ \gamma_{M} \circ F\left(i_{\tau M}\right)\right) & =\operatorname{Im}\left(f \circ \gamma_{M} \circ F\left(i_{\tau^{\prime} M}\right) \circ F(j)\right) \\
& \leq \operatorname{Im}\left(f \circ \gamma_{M} \circ F\left(i_{\tau^{\prime} M}\right)\right) .
\end{aligned}
$$

Por lo que $\alpha_{\gamma_{M} \circ F\left(i_{\tau M}\right)}(K) \leq \alpha_{\gamma_{M} \circ F\left(i_{\tau^{\prime} M}\right)}(K)$ y por tanto $\varphi(\tau) \preceq \varphi\left(\tau^{\prime}\right)$. Así que $\varphi_{\gamma}$ preserva el orden y por lo tanto es un morfismo de clapos.

Ahora veamos que $\psi_{\gamma}$ preserva el orden. Sean $\tau, \tau^{\prime} \in R$-pr tal que $\tau \preceq \tau^{\prime}$. Probemos que $\psi_{\gamma}(\tau) \preceq \psi_{\gamma}\left(\tau^{\prime}\right)$. Basta demostrar que para toda $M \in R$-Mod, $\omega_{\gamma_{(M / \tau M)} \circ F\left(p_{\tau M}\right)} \preceq \omega_{\gamma_{\left(M / \tau^{\prime} M\right)^{\circ}} \circ F\left(p_{\tau^{\prime} M}\right)}$, donde $p_{\tau^{\prime} M}: M \rightarrow M / \tau^{\prime}(M)$ es la proyección natural. Sean $M \in R$-Mod y $K \in S$-Mod. Entonces, por la Definición 2.48:

$$
\omega_{\gamma_{(M / \tau M)} \circ F(p \tau M)}(K)=\bigcap\left\{\overleftarrow{f}\left(\operatorname{ker}\left(\gamma_{(M / \tau M)} \circ F\left(p_{\tau M}\right)\right)\right) \mid f: K \rightarrow F(M)\right\}
$$

$\mathrm{y}$

$$
\omega_{\gamma_{\left(M / \tau^{\prime} M\right)} \circ F\left(p \tau^{\prime} M\right)}(K)=\bigcap\left\{\overleftarrow{g}\left(\operatorname{ker}\left(\gamma_{\left(M / \tau^{\prime} M\right)} \circ F\left(p_{\tau^{\prime} M}\right)\right)\right) \mid g: K \rightarrow F(M)\right\}
$$

Se tiene que $p_{\tau^{\prime} M} \circ i_{\tau^{\prime} M}=0$, donde $i_{\tau^{\prime} M}: \tau^{\prime}(M) \hookrightarrow M$ es la inclusión natural. Entonces por el Teorema del Factor existe $h: M / \tau M \rightarrow M / \tau^{\prime} M$ de manera única tal que el siguiente diagrama es conmutativo:

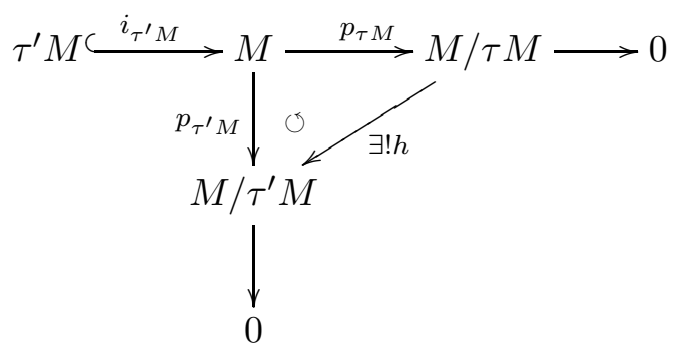

Sea $g: K \rightarrow F(M)$. Consideremos el siguiente diagrama:

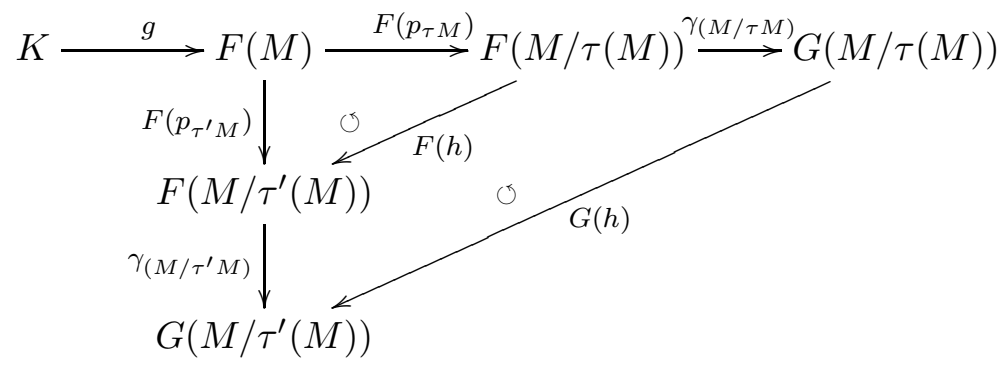


donde el triángulo es conmutativo puesto que es aplicar el funtor $F$ al diagrama (35) y el rectángulo conmuta por la naturalidad de $\gamma$. De ahí

$$
\begin{aligned}
\overleftarrow{g}\left(\operatorname{ker}\left(\gamma_{\left(M / \tau^{\prime} M\right)} \circ F\left(p_{\tau^{\prime} M}\right)\right)\right) & =\operatorname{ker}\left(\gamma_{\left(M / \tau^{\prime} M\right)} \circ F\left(p_{\tau^{\prime} M}\right) \circ g\right) \\
& =\operatorname{ker}\left(G(h) \circ \gamma_{(M / \tau M)} \circ F\left(p_{\tau M}\right) \circ g\right) \\
& \geq \operatorname{ker}\left(\gamma_{(M / \tau M)} \circ F\left(p_{\tau M}\right) \circ g\right) \\
& =\overleftarrow{g}\left(\operatorname{ker}\left(\gamma_{(M / \tau M)} \circ F\left(p_{\tau M}\right)\right)\right) \\
& \geq \omega_{\gamma_{(M / \tau M)} \circ F\left(p_{\tau M}\right)}(K)
\end{aligned}
$$

Es decir, $\omega_{\gamma_{(M / \tau M)} \circ F\left(p_{\tau M}\right)}(K) \leq \overleftarrow{g}\left(\operatorname{ker}\left(\gamma_{\left(M / \tau^{\prime} M\right)} \circ F\left(p_{\tau^{\prime} M}\right)\right)\right)$ para toda $g$ $K \rightarrow F(M)$. Por lo tanto $\omega_{\gamma_{(M / \tau M)} \circ F\left(p_{\tau M}\right)}(K) \leq \omega_{\gamma_{\left(M / \tau^{\prime} M\right)} \circ F\left(p_{\tau^{\prime} M}\right)}(K)$. Entonces $\psi_{\gamma}(\tau) \preceq \psi_{\gamma}\left(\tau^{\prime}\right)$. Concluimos que $\psi_{\gamma}$ preserva el orden y por tanto es un morfismo de clapos.

En adelante en algunas ocasiones denotaremos a la composición de transformaciones naturales $\gamma: F \rightarrow G$ y $\delta: G \rightarrow H$ con $F, G, H: R$-Mod $\rightarrow S$-Mod funtores, como $\delta \circ \gamma=\delta \gamma$.

Proposición 3.3. Sean $F, G, H: R$-Mod $\rightarrow S$-Mod funtores, $\gamma: F \rightarrow G y$ $\delta: G \rightarrow H$ transformaciones naturales. Entonces para $\tau \in R$-pr:

1. $\varphi_{\delta \gamma}(\tau) \preceq \varphi_{\delta}(\tau), \varphi_{\delta \gamma}(\tau) \preceq \varphi_{\gamma}(\tau)$.

2. $\psi_{\delta \gamma}(\tau) \succeq \psi_{\delta}(\tau), \psi_{\delta \gamma}(\tau) \succeq \psi_{\gamma}(\tau)$.

Demostración. 1. Sea $\tau \in R$-pr. Probemos que $\varphi_{\delta \gamma}(\tau) \preceq \varphi_{\delta}(\tau)$. Por lo que veremos que para cada $M \in R$-Mod, $\alpha_{(\delta \circ \gamma)_{M} \circ F\left(i_{\tau M}\right)} \preceq \alpha_{\delta_{M} \circ G\left(i_{\tau M}\right)}$, donde $i_{\tau M}: \tau(M) \hookrightarrow M$ es la inclusión natural. Sean $M \in R$-Mod, $K \in S$-Mod, $f: H(M) \rightarrow K$ y consideremos el siguiente diagrama conmutativo:

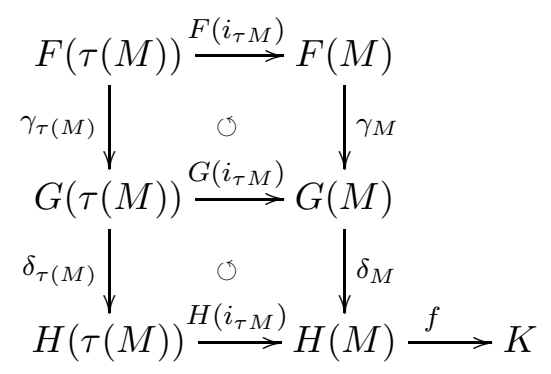

Del Diagrama (36) :

$$
\begin{aligned}
f\left(\operatorname{Im}(\delta \circ \gamma)_{M} \circ F\left(i_{\tau M}\right)\right) & =\operatorname{Im}\left(f \circ \delta_{M} \circ G\left(i_{\tau M}\right) \circ \gamma_{\tau(M)}\right) \\
& \leq \operatorname{Im}\left(f \circ \delta_{M} \circ G\left(i_{\tau M}\right)\right) \\
& =f\left(\operatorname{Im} \delta_{M} \circ G\left(i_{\tau M}\right)\right) \leq \alpha_{\delta_{M} \circ G\left(i_{\tau M}\right)}(K)
\end{aligned}
$$

Por lo tanto para cada $M \in R$-Mod, se tiene que $\alpha_{(\delta \circ \gamma)_{M} \circ F\left(i_{\tau M}\right)} \preceq \alpha_{\delta_{M} \circ G\left(i_{\tau M}\right)}$, de donde, $\varphi_{\delta \gamma}(\tau) \preceq \varphi_{\delta}(\tau)$. 
Ahora mostraremos que $\varphi_{\delta \gamma}(\tau) \preceq \varphi_{\gamma}(\tau)$. Por lo que veremos que para cada $M \in R$-Mod, $\alpha_{(\delta \circ \gamma)_{M} \circ F\left(i_{\tau M}\right)} \preceq \alpha_{\gamma_{M} \circ F\left(i_{\tau M}\right)}$. Sean $M \in R$-Mod, $K \in S$-Mod, $f: H(M) \rightarrow K$. Entonces:

$$
\begin{aligned}
f\left(\operatorname{Im}(\delta \circ \gamma)_{M} \circ F\left(i_{\tau M}\right)\right) & =\operatorname{Im}\left(\left(f \circ \delta_{M}\right) \circ \gamma_{M} \circ F\left(i_{\tau M}\right)\right) \\
& =g\left(\operatorname{Im} \gamma_{M} \circ F\left(i_{\tau M}\right)\right),
\end{aligned}
$$

donde $g=f \circ \delta_{M}: G(M) \rightarrow K$. Por lo tanto $\varphi_{\delta \gamma}(\tau) \preceq \varphi_{\gamma}(\tau)$.

2. Ahora veamos que $\psi_{\delta \gamma}(\tau) \succeq \psi_{\delta}(\tau)$. Entonces probaremos que para cada $M \in R$-Mod, $\omega_{\delta_{(M / \tau M)} \circ F\left(p_{\tau M}\right)} \preceq \omega_{(\delta \circ \gamma)_{M / \tau M} \circ F\left(p_{\tau M}\right)}$, donde $p_{\tau M}: M \rightarrow$ $M / \tau(M)$ es la proyección natural. Sean $M \in R$-Mod, $K \in S$-Mod y $f: K \rightarrow$ $F(M)$. Consideremos el siguiente diagrama conmutativo:

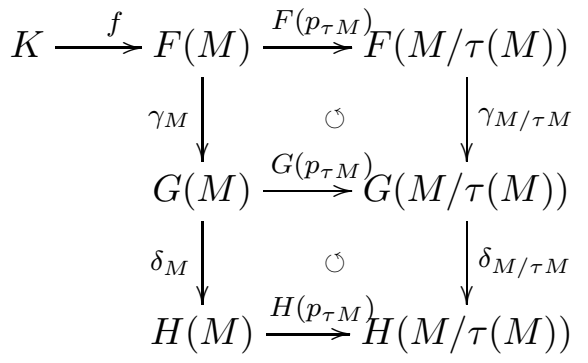

De donde:

$$
\begin{aligned}
& \overleftarrow{f}\left(\operatorname{ker}(\delta \circ \gamma)_{M / \tau M} \circ F\left(p_{\tau M}\right)\right)=\operatorname{ker}\left((\delta \circ \gamma)_{M / \tau M} \circ F\left(p_{\tau M}\right) \circ f\right) \\
&=\operatorname{ker}\left(\delta_{(M / \tau M)} \circ G\left(p_{\tau M}\right) \circ \gamma_{M} \circ f\right) \\
&=\overleftarrow{\gamma_{M} \circ f}\left(\operatorname{ker} \delta_{M / \tau M} \circ G\left(p_{\tau M}\right)\right) \\
& \geq \omega_{\delta_{(M / \tau M)}} \circ G\left(p_{\tau M}\right) \\
&
\end{aligned}
$$

De ahí se concluye que $\psi_{\delta \gamma}(\tau) \succeq \psi_{\delta}(\tau)$.

Del mismo modo veamos que $\psi_{\delta \gamma}(\tau) \succeq \psi_{\gamma}(\tau)$. Sea $\tau \in R$-Mod. Entonces probaremos que para cada $M \in R$-Mod, $\omega_{\gamma_{(M / \tau M)} \circ F\left(p_{\tau M}\right)} \preceq \omega_{(\delta \circ \gamma)_{M / \tau M} \circ F\left(p_{\tau M}\right)}$, donde $p_{\tau M}: M \rightarrow M / \tau(M)$ es la proyección natural. Sean $M \in R$-Mod, $K \in S$ Mod y $f: K \rightarrow F(M)$. Entonces

$$
\begin{aligned}
\overleftarrow{f}\left(\operatorname{ker}(\delta \circ \gamma)_{M / \tau M} \circ F\left(p_{\tau M}\right)\right) & =\operatorname{ker}\left((\delta \circ \gamma)_{M / \tau M} \circ F\left(p_{\tau M}\right) \circ f\right) \\
& \geq \operatorname{ker}\left(\gamma_{(M / \tau M)} \circ F\left(p_{\tau M}\right) \circ f\right) \\
& =\overleftarrow{f}\left(\operatorname{ker} \gamma_{(M / \tau M)} \circ F\left(p_{\tau M}\right)\right) \\
& \geq \omega_{\gamma_{(M / \tau M)} \circ F\left(p_{\tau M}\right)}(K)
\end{aligned}
$$

Por lo tanto, $\psi_{\delta \gamma}(\tau) \succeq \psi_{\gamma}(\tau)$.

El siguiente corolario es inmediato de la Proposición 3.3.

Corolario 3.4. Sean F, $G, H: R$-Mod $\rightarrow S$-Mod funtores, $\gamma: F \rightarrow G y \delta: G \rightarrow$ $H$ transformaciones naturales. Entonces para $\tau \in R$-pr: 
1. $\varphi_{\delta \gamma}(\tau) \preceq \varphi_{\delta}(\tau) \bigwedge \varphi_{\gamma}(\tau)$.

2. $\psi_{\delta \gamma}(\tau) \succeq \psi_{\delta}(\tau) \bigvee \psi_{\gamma}(\tau)$

Corolario 3.5. Sean F, $G, H: R$-Mod $\rightarrow S$-Mod funtores, $\gamma: F \rightarrow G y \delta: G \rightarrow$ $H$ transformaciones naturales. Entonces

1. Si $\delta$ es un isomorfismo natural, entonces $\varphi_{\delta \gamma}=\varphi_{\gamma}$.

2. Si $\gamma$ es un isomorfismo natural, entonces $\varphi_{\delta \gamma}=\varphi_{\delta}$.

Demostración. 1. Sea $\tau \in R$-pr. Para cada $M \in R$-Mod, tenemos que $\delta_{M}$ : $G(M) \rightarrow H(M)$ es un isomorfismo. Así que en el Diagrama (36), se puede agregar el morfismo $\delta_{M}^{-1}: H(M) \rightarrow G(M)$. Sea $g: G(M) \rightarrow K$ y viendo el diagrama (36), obtenemos:

$$
\begin{aligned}
\operatorname{Im}\left(g \circ \gamma_{M} \circ F\left(i_{\tau M}\right)\right) & =\operatorname{Im}\left(g \circ \delta_{M}^{-1} \circ H\left(i_{\tau M}\right) \circ \delta_{\tau M} \circ \gamma_{\tau M}\right) \\
& =\operatorname{Im}\left(g \circ \delta_{M}^{-1} \circ \delta_{M} \circ \gamma_{M} \circ F\left(i_{\tau M}\right)\right) \\
& =g \circ \delta_{M}^{-1}\left(\operatorname{Im}(\delta \circ \gamma)_{M} \circ F\left(i_{\tau M}\right)\right)
\end{aligned}
$$

De ahí, $\varphi_{\gamma}(\tau) \preceq \varphi_{\delta \gamma}(\tau)$. Así que por 1 de la Proposición 3.3, concluimos que $\varphi_{\delta \gamma}=\varphi_{\gamma}$

2. Del mismo modo, sea $\tau \in R$-pr. Se tiene que para cada $M \in R$-Mod, $\gamma_{M}: F(M) \rightarrow G(M)$ es un ismorfismo. Así que en el Diagrama (36), se puede agregar el $\gamma_{\tau M}^{-1}: G(\tau(M)) \rightarrow F(\tau(M))$. Sea $f: H(M) \rightarrow K$ y del diagrama (36), se tiene:

$$
\begin{aligned}
\operatorname{Im}\left(f \circ \delta_{M} \circ G\left(i_{\tau M}\right)\right) & =\operatorname{Im}\left(f \circ \delta_{M} \circ \gamma_{M} \circ F\left(i_{\tau M}\right) \circ \gamma_{\tau M}^{-1}\right) \\
& \leq \operatorname{Im}\left(f \circ \delta_{M} \circ \gamma_{M} \circ F\left(i_{\tau M}\right)\right)
\end{aligned}
$$

De donde, $\varphi_{\delta}(\tau) \preceq \varphi_{\delta \gamma}(\tau)$. Así que por 1 de la Proposición 3.3 obtenemos que $\varphi_{\delta \gamma}=\varphi_{\delta}$

Corolario 3.6. Sean F, $G, H: R$-Mod $\rightarrow S$-Mod funtores, $\gamma: F \rightarrow G$ y $\delta: G \rightarrow$ $H$ transformaciones naturales. Entonces

1. Si $\delta$ es un isomorfismo natural, entonces $\psi_{\delta \gamma}=\psi_{\gamma}$.

2. Si $\gamma$ es un isomorfismo natural, entonces $\psi_{\delta \gamma}=\psi_{\delta}$.

Demostración. 1. Sea $\tau \in R$-pr. Para cada $M \in R$-Mod, $\delta_{M}$ es un isomorfismo. Entonces, sea $f: K \rightarrow F(M)$, con $K \in S$-Mod. En el Diagrama (37), se puede agregar el morfismo $\delta_{M / \tau M}^{-1}: H(M \tau M) \rightarrow G(M / \tau M)$ y así se tiene:

$$
\begin{aligned}
\operatorname{ker}\left(\gamma_{M / \tau M} \circ F\left(p_{\tau M}\right) \circ f\right) & =\operatorname{ker}\left(\delta_{M / \tau M}^{-1} \circ \delta_{M / \tau M} \circ \gamma_{M / \tau M} \circ F\left(p_{\tau M}\right) \circ f\right) \\
& \geq \operatorname{ker}\left(\delta_{M / \tau M} \circ \gamma_{M / \tau M} \circ F\left(p_{\tau M}\right) \circ f\right) \\
& =\overleftarrow{f}\left(\operatorname{ker}(\delta \circ \gamma)_{M / \tau M} \circ F\left(p_{\tau M}\right)\right)
\end{aligned}
$$


De donde obtenemos que $\psi_{\gamma}(\tau) \succeq \psi_{\delta \gamma}(\tau)$ y por 2 de la Proposición 3.3, concluimos que $\psi_{\delta \gamma}=\psi_{\gamma}$.

2. Sea $\tau \in R$-pr. Para cada $M \in R$-Mod, $\gamma_{M}$ es un isomorfismo. Sea $g: K \rightarrow$ $G(M)$, con $K \in S$-Mod. En el Diagrama (37), se puede agregar el morfismo $g$ y el morfismo $\gamma_{M}^{-1}$, de donde tenemos lo siguiente:

$$
\begin{aligned}
\operatorname{ker}\left(\delta_{M / \tau M} \circ G\left(p_{\tau M}\right) \circ g\right) & =\operatorname{ker}\left(\delta_{M / \tau M} \circ \gamma_{M / \tau M} \circ F\left(p_{\tau M}\right) \circ \gamma_{M}^{-1} \circ g\right) \\
& =\overleftarrow{\gamma_{M}^{-1} \circ g}\left(\operatorname{ker}(\delta \circ \gamma)_{M / \tau M} \circ F\left(p_{\tau M}\right)\right)
\end{aligned}
$$

Puesto que $\gamma_{M}^{-1} \circ g: K \rightarrow F(M)$, obtenemos que $\psi_{\delta}(\tau) \succeq \psi_{\delta \gamma}(\tau)$. Luego, de la Proposición 3.3 parte 2 , concluimos que $\psi_{\delta \gamma}=\psi_{\delta}$.

En el siguiente corolario analizaremos a los prerradicales en $S$-pr: $\varphi_{\gamma}\left(0_{R}\right)$, $\varphi_{\gamma}\left(1_{R}\right), \psi_{\gamma}\left(0_{R}\right)$ y $\psi_{\gamma}\left(1_{R}\right)$.

Corolario 3.7. Sean F, $G: R$-Mod $\rightarrow$ S-Mod funtores $y \gamma: F \rightarrow G$ una transformación natural. Entonces

1. $\varphi_{\gamma}\left(1_{R}\right)=\bigvee_{M \in R \text {-Mod }} \alpha_{I m \gamma_{M}}^{G(M)}$. Si $\gamma$ es un epimorfismo natural, entonces $\varphi_{\gamma}\left(1_{R}\right)$ es un prerradical idempotente en $S$-pr.

2. $\varphi_{\gamma}\left(0_{R}\right)=\bigvee_{M \in R-M o d} \alpha_{I m \gamma_{M} \circ F\left(i_{0_{R} M}\right)}^{G(M)}$.

3. $\psi_{\gamma}\left(1_{R}\right)=\bigwedge_{M \in R \text {-Mod }} \omega_{\text {ker } \gamma_{0} \circ F\left(p_{M}\right)}^{F(M)}$, donde $0 \in R$-Mod y $p_{M}: M \rightarrow 0$.

4. $\psi_{\gamma}\left(0_{R}\right)=\bigwedge_{M \in R-M o d} \omega_{k e r \gamma_{M}}^{F(M)}$. Si $\gamma$ es un monomorfismo natural, entonces $\psi_{\gamma}\left(0_{R}\right)$ es un radical en $S$-pr.

DEMOSTRACIÓN.

1. Sea $1_{R}: R$-Mod $\rightarrow R$-Mod el prerradical identidad. Entonces por 1, de la Definición 3.1:

$$
\left.\varphi_{\gamma}\left(1_{R}\right)=\bigvee_{M \in R \text {-Mod }} \alpha_{\gamma_{M} \circ F\left(i_{1_{R}}(M)\right.}\right)=\bigvee_{M \in R \text {-Mod }} \alpha_{\gamma_{M}}=\bigvee_{M \in R \text {-Mod }} \alpha_{\operatorname{Im} \gamma_{M}}^{G(M)}
$$

$\mathrm{Si} \gamma$ es un epimorfismo natural, entonces

$$
\varphi_{\gamma}\left(1_{R}\right)=\bigvee_{M \in R-\operatorname{Mod}} \alpha_{G(M)}^{G(M)}
$$

es un prerradical idempotente.

2. Sea $0_{R}: R$-Mod $\rightarrow R$-Mod el prerradical cero. Entonces

$$
\varphi_{\gamma}\left(0_{R}\right)=\bigvee_{M \in R \text {-Mod }} \alpha_{\gamma_{M} \circ F\left(i_{0_{R}(M)}\right)}=\bigvee_{M \in R \text {-Mod }} \alpha_{\operatorname{Im} \gamma_{M} \circ F\left(i_{0_{R} M}\right)}^{G(M)}
$$

3. Por la Definición 3.1:

$$
\psi_{\gamma}\left(1_{R}\right)=\bigwedge_{M \in R \text {-Mod }} \omega_{\gamma_{M / 1_{R} M^{M}} \circ F\left(p_{1_{R}(M)}\right)}=\bigwedge_{M \in R \text {-Mod }} \omega_{\mathrm{ker} \gamma_{0} \circ F\left(p_{M}\right)}^{F(M)} .
$$


4. Por la Definición 3.1:

$$
\psi_{\gamma}\left(0_{R}\right)=\bigwedge_{M \in R \text {-Mod }} \omega_{\gamma_{M / 0_{R} M} \circ F\left(p_{0_{R}(M)}\right)}=\bigwedge_{M \in R \text {-Mod }} \omega_{\gamma_{M}}=\bigwedge_{M \in R \text {-Mod }} \omega_{\mathrm{ker} \gamma_{M}}^{F(M)},
$$

donde $p_{0_{R}(M)}: M \rightarrow M$. Si $\gamma: F \rightarrow G$ es un monomorfismo natural, entonces

$$
\psi_{\gamma}\left(0_{R}\right)=\bigwedge_{M \in R-\operatorname{Mod}} \omega_{0}^{F(M)}
$$

es un radical.

Observación 3.8. En el Corolario 3.7 parte 2, si $F$ es un un funtor que preserva morfismos cero, entonces $\varphi_{\gamma}\left(0_{R}\right)=0_{S}$. En particular, cuando $F$ es un prerradical ó cuando $F$ es un funtor aditivo, también se tiene que $\varphi_{\gamma}\left(0_{R}\right)=0_{S}$.

En 3 del Corolario 3.7, si G es un funtor que preserva morfismos cero, entonces

$$
\psi_{\gamma}\left(1_{R}\right)=\bigwedge_{M \in R-M o d} \omega_{F(M)}^{F(M)}=1_{S}
$$

A los prerradicales $\varphi_{\gamma}\left(1_{R}\right)$ y $\psi_{\gamma}\left(0_{R}\right)$, los denotaremos de la siguiente manera.

Notación. Sean $F, G: R$-Mod $\rightarrow S$-Mod funtores y $\gamma: F \rightarrow G$ una transformación natural. Denotaremos a los prerradicales $\varphi_{\gamma}\left(1_{R}\right)$ y $\psi_{\gamma}\left(0_{R}\right)$ como:

$$
\varphi_{\gamma}\left(1_{R}\right)=\widehat{\varepsilon}_{\gamma} \text { y } \psi_{\gamma}\left(0_{R}\right)=\widehat{\eta}_{\gamma} .
$$

\section{2. $\varphi_{\gamma} \mathbf{y} \psi_{\gamma}$ para transformaciones naturales $\gamma$ específicas}

En esta sección veremos algunos ejemplos de morfismos entre retículas de prerradicales asociados a ciertas transformaciones naturales que en particular involucren prerradicales.

Proposición 3.9. Sea $\sigma \in R$-pr y consideremos la transformación natural $i_{\sigma}$ : $\sigma \hookrightarrow 1_{R}$. Entonces $\varphi_{i_{\sigma}}(\tau)=\sigma \tau$, para $\tau \in R$-pr.

Demostración. El resultado se sigue del Teorema 1.58 y de las Definiciones 2.48 y 3.1, puesto que para $\tau \in R$-pr:

$$
\varphi_{i_{\sigma}}(\tau)=\bigvee_{M \in R \text {-Mod }} \alpha_{\left(i_{\sigma}\right)_{M} \circ \sigma\left(i_{\tau M}\right)}=\bigvee_{M \in R \text {-Mod }} \alpha_{i_{\sigma \tau(M)}}=\bigvee_{M \in R \text {-Mod }} \alpha_{\sigma \tau(M)}^{M}=\sigma \tau,
$$

donde $i_{\sigma \tau(M)}: \sigma \tau(M) \hookrightarrow M$.

El siguiente resultado es el dual de la Proposición 3.9, en donde trabajamos con el coprerradical $\sigma^{*}$, el cual presentamos en la Definición 2.39.

Proposición 3.10. Sea $\sigma \in R$-pr y consideremos la transformación natural $p_{\sigma^{*}}$ : $1_{R} \rightarrow \sigma^{*}$. Entonces $\psi_{p_{\sigma^{*}}}(\tau)=(\tau: \sigma)$, para $\tau \in R$-pr. 
Demostración. Sea $\tau \in R$-pr. Entonces, por la Definición 3.1, $\psi_{p_{\sigma^{*}}}(\tau)=\bigwedge_{M \in R \text {-Mod }} \omega_{p_{\sigma_{(M / \tau M)}^{*}} \circ 1\left(p_{\tau M}\right)}$, donde $p_{\tau M}: M \rightarrow M / \tau(M)$. Luego, para cada $M \in R$-Mod, $p_{\sigma_{(M / \tau M)}^{*}}: M / \tau(M) \rightarrow(M / \tau(M)) / \sigma(M \tau(M))$. Por la Definición 1.62, $\sigma(M / \tau(M))=(\tau: \sigma)(M) / \tau(M)$, por lo tanto $p_{\sigma_{(M / \tau M)}^{*}} \circ 1\left(p_{\tau M}\right)$ : $M \rightarrow M /(\tau: \sigma)(M)$. Así,

$$
\psi_{p_{\sigma^{*}}}(\tau)=\bigwedge_{M \in R \text {-Mod }} \omega_{\operatorname{ker}\left(p_{\sigma_{(M / \tau M)}^{*}}^{M} \circ 1\left(p_{\tau M}\right)\right)}=\bigwedge_{M \in R \text {-Mod }} \omega_{(\tau: \sigma)(M)}^{M}=(\tau: \sigma) .
$$

Donde la última igualdad se tiene del Teorema 1.58. Por lo tanto concluimos que $\psi_{p_{\sigma^{*}}}(\tau)=(\tau: \sigma)$.

Observación 3.11. Sea $\sigma \in R$-pr. Notemos que de las Proposiciones 3.9 y 3.10, se tienen respectivamente los operadores siguientes:

1. $\varphi_{i_{\sigma}}(-): R-p r \rightarrow R-p r$, tal que $\varphi_{i_{\sigma}}(-)=\sigma \cdot \cdot_{-}$.

2. $\psi_{p_{\sigma^{*}}}(-): R-p r \rightarrow R-p r$, tal que $\psi_{p_{\sigma^{*}}}\left({ }_{-}\right)=(-: \sigma)$.

Proposición 3.12. Sean $\rho, \sigma \in R$-pr tal que $\rho \preceq \sigma$ y consideremos la transformación natural $i_{\rho, \sigma}: \rho \hookrightarrow \sigma$. Entonces $\rho \tau \preceq \varphi_{i_{\rho, \sigma}}(\tau)$, para $\tau \in R$-pr.

Demostración. Sea $\tau \in R$-pr. Entonces el resultado se obtiene de las Proposiciones 3.3 y 3.9 , puesto que:

$$
\rho \tau=\varphi_{i_{\rho}}(\tau)=\varphi_{i_{\sigma} \circ i_{\rho, \sigma}}(\tau) \preceq \varphi_{i_{\rho, \sigma}}(\tau) .
$$

Para el dual de la Proposición 3.12, usaremos la siguiente transformación natural:

Sean $\rho, \sigma \in R$-pr, tal que $\rho \preceq \sigma$. Entonces definimos la transformación natural $p_{\rho, \sigma}: \rho^{*} \rightarrow \sigma^{*}$ tal que para cada $M \in R$-Mod consideramos el morfismo $p_{\rho, \sigma_{(M)}}: M / \rho(M) \rightarrow M / \sigma(M)$, el cual existe por el Teorema del Factor usando un diagrama similar a (18) de la Sección 2.1. Tales morfismos cumplen la condición de naturalidad para cada morfimo en $R$-Mod.

Proposición 3.13. Sean $\rho, \sigma \in R$-pr tal que $\rho \preceq \sigma$ y consideremos la transformación natural $p_{\rho, \sigma}: \rho^{*} \hookrightarrow \sigma^{*}$. Entonces $(\tau: \sigma) \succeq \psi_{p_{\rho, \sigma}}(\tau)$, para $\tau \in R$-pr.

Demostración. Sea $\tau \in R$-pr. Por las Proposiciones 3.3 y 3.10 se tiene que

$$
(\tau: \sigma)=\psi_{p_{\sigma^{*}}}(\tau)=\psi_{p_{\rho, \sigma} \circ p_{\rho^{*}}}(\tau) \succeq \psi_{p_{\rho, \sigma}}(\tau) .
$$

Proposición 3.14. Sean $\sigma, \rho \in R$-pr tal que $\rho \preceq \sigma$ y consideremos la transformación natural $i_{\rho, \sigma}: \rho \hookrightarrow \sigma$. Entonces para $\tau \in R$-pr

$$
\varphi_{i_{\rho, \sigma}}(\tau)=\bigwedge\{\eta \in R-p r \mid \rho \tau \preceq \eta \sigma\}
$$


Demostración. Sea $\tau \in R$-pr. Por la Definición 3.1, se tiene que $\varphi_{i_{\rho, \sigma}}(\tau)=$ $\bigvee_{M \in R \text {-Mod }} \alpha_{\rho(\tau(M))}^{\sigma(M)}$. Primero probemos que $\rho \tau \preceq \varphi_{i_{\rho, \sigma}}(\tau) \sigma$. Sea $K \in R$-Mod. Entonces por 3 del Teorema 1.63:

$$
\begin{aligned}
\left(\varphi_{i_{\rho, \sigma}}(\tau) \sigma\right)(K) & =\bigvee_{M \in R \text {-Mod }}\left(\alpha_{\rho(\tau(M))}^{\sigma(M)} \sigma\right)(K) \\
& \geq\left(\alpha_{\rho(\tau(K))}^{\sigma(K)} \sigma\right)(K)=\alpha_{\rho(\tau(K))}^{\sigma(K)}(\sigma(K)) \geq 1_{\sigma K}(\rho(\tau(K))) \\
& \rho(\tau(K))
\end{aligned}
$$

donde $1_{\sigma K}: \sigma(K) \rightarrow \sigma(K)$ es el morfismo identidad. De donde $\rho \tau \preceq \varphi_{i_{\rho, \sigma}}(\tau) \sigma$. Por lo tanto $\bigwedge\{\eta \in R$-pr $\mid \rho \tau \preceq \eta \sigma\} \preceq \varphi_{i_{\rho, \sigma}}(\tau)$.

Para la otra desigualdad, probemos que para cada $M \in R$-Mod se cumple que

$$
\alpha_{\rho(\tau(M))}^{\sigma(M)} \preceq \bigwedge\{\eta \in R \text {-pr } \mid \rho \tau \preceq \eta \sigma\} .
$$

Sean $M, K \in R$-Mod y $\eta \in R$-pr, tal que $\rho \tau \preceq \eta \sigma$. Se tiene que

$$
\alpha_{\rho(\tau(M))}^{\sigma(M)}(K)=\sum\{f(\rho(\tau(M))) \mid f: \sigma(M) \rightarrow K\},
$$

pero $f(\rho(\tau(M))) \leq f(\eta(\sigma(M))) \leq \eta(M)$ puesto que $\eta$ es un $R$-prerradical. Por lo tanto $\alpha_{\rho(\tau(M))}^{\sigma(M)} \preceq \eta$ para toda $\eta \in R$-pr tal que $\rho \tau \preceq \eta \sigma$, de donde se concluye (39). Entonces

$$
\varphi_{i_{\rho, \sigma}}(\tau)=\bigvee_{M \in R \text {-Mod }} \alpha_{\rho(\tau(M))}^{\sigma(M)} \preceq \bigwedge\{\eta \in R \text {-pr } \mid \rho \tau \preceq \eta \sigma\} .
$$

Concluimos que $\varphi_{i_{\rho, \sigma}}(\tau)=\bigwedge\{\eta \in R$-pr $\mid \rho \tau \preceq \eta \sigma\}$, para $\tau \in R$-pr.

\section{Observación 3.15.}

$$
\varphi_{i_{\rho, \sigma}}\left(1_{R}\right)=\bigwedge\{\eta \in R-p r \mid \rho \preceq \eta \sigma \preceq \sigma\} .
$$

Proposición 3.16. Sean $\sigma, \rho \in R$-pr tal que $\rho \preceq \sigma$ y consideremos la transformación natural $p_{\rho, \sigma}: \rho^{*} \rightarrow \sigma^{*}$. Entonces para $\tau \in R$-pr

$$
\psi_{p_{\rho, \sigma}}(\tau)=\bigvee\{\eta \in R-p r \mid(\rho: \eta) \preceq(\tau: \sigma)\}
$$

Demostración. Sea $\tau \in R$-pr. Por la Definición 3.1, tenemos que $\psi_{p_{\rho, \sigma}}(\tau)=$ $\bigwedge_{M \in R \text {-Mod }} \omega_{(\tau: \sigma)(M) / \rho(M)}^{M / \rho(M)}$. Primero probaremos que

$$
\bigvee\{\eta \in R \text {-pr } \mid(\rho: \eta) \preceq(\tau: \sigma)\} \preceq \bigwedge_{M \in R \text {-Mod }} \omega_{(\tau: \sigma)(M) / \rho(M)}^{M / \rho(M)} .
$$

Para esto, mostraremos que

$$
\bigvee\{\eta \in R \text {-pr } \mid(\rho: \eta) \preceq(\tau: \sigma)\} \preceq \omega_{(\tau: \sigma)(M) / \rho(M)}^{M / \rho(M)},
$$

para toda $M \in R$-Mod. 
Sean $M, K \in R$-Mod y $\eta \in R$-pr, tal que $(\rho: \eta) \preceq(\tau: \sigma)$. Se tiene que

$$
\omega_{(\tau: \sigma)(M) / \rho(M)}^{M / \rho(M)}(K)=\bigcap\{\overleftarrow{g}((\tau: \sigma)(M) / \rho(M)) \mid g: K \rightarrow M / \rho(M)\}
$$

Para toda $g: K \rightarrow M / \rho(M)$, tenemos que $g(\eta(K)) \leq \eta(M / \rho(M))$, así que

$$
\eta(K) \leq \overleftarrow{g}(\eta(M / \rho(M)))=\overleftarrow{g}((\rho: \eta) / \rho(M)) \leq \overleftarrow{g}((\tau: \sigma)(M) / \rho(M))
$$

De lo anterior obtenemos que $\eta(K) \leq \omega_{(\tau: \sigma)(M) / \rho(M)}^{M / \rho(M)}(K)$. Por lo tanto $\eta \preceq \omega_{(\tau: \sigma)(M) / \rho(M)}^{M / \rho(M)}$, para toda $\eta \in R$-pr, tal que $(\rho: \eta) \preceq(\tau: \sigma)$. De donde, se obtiene (40) y por tanto se conluye $\bigvee\{\eta \in R$-pr $\mid(\rho: \eta) \preceq(\tau: \sigma)\} \preceq \psi_{p_{\rho, \sigma}}(\tau)$.

Para la otra contención, sea $\tau \in R$-pr y veamos que $\left(\rho: \psi_{p_{\rho, \sigma}}(\tau)\right) \preceq(\tau: \sigma)$. Sea $K \in R$-Mod. Por 5 del Teorema 1.63:

$$
\left(\rho: \psi_{p_{\rho, \sigma}}(\tau)\right)(K)=\bigwedge_{M \in R \text {-Mod }}\left(\rho: \omega_{(\tau: \sigma)(M) / \rho(M)}^{M / \rho(M)}\right)(K) \leq\left(\rho: \omega_{(\tau: \sigma)(K) / \rho(K)}^{K / \rho(K)}\right)(K) .
$$

Se tiene que $\omega_{(\tau: \sigma)(K) / \rho(K)}^{K / \rho(K)}(K / \rho(K)) \subseteq \overleftarrow{g}((\tau: \sigma)(K) / \rho(K))$, para toda $g$ $K / \rho(K) \rightarrow K / \rho(K)$. De donde $\left(\rho: \omega_{(\tau: \sigma)(K) / \rho(K)}^{K / \rho(K)}\right)(K) \leq(\tau: \sigma)(K)$. Por lo tanto, $\psi_{p_{\rho, \sigma}}(\tau) \preceq \bigvee\{\eta \in R$-pr $\mid(\rho: \eta) \preceq(\tau: \sigma)\}$. Concluimos entonces:

$$
\psi_{p_{\rho, \sigma}}(\tau)=\bigvee\{\eta \in R \text {-pr } \mid(\rho: \eta) \preceq(\tau: \sigma)\},
$$

para $\tau \in R$-pr.

Concluiremos esta sección con los morfismos $\varphi$ y $\psi$ para un producto estrella de transformaciones naturales. Para lo cual primero veamos lo siguiente:

Sean $F, G: R$-Mod $\rightarrow S$-Mod y $H, K: S$-Mod $\rightarrow T$-Mod funtores. Consideremos $\delta: F \rightarrow G$ y $\gamma: H \rightarrow K$ transformaciones naturales. Entonces, de acuerdo a la Definición 1.29, $\gamma * \delta: H \circ F \rightarrow K \circ G$ tal que para cada $M \in R$ Mod, $(\gamma * \delta)_{M}:(H \circ F)(M) \rightarrow(K \circ G)(M)$ se define como $(\gamma * \delta)_{M}:=$ $K\left(\delta_{M}\right) \circ \gamma_{F(M)}=\gamma_{G(M)} \circ H\left(\delta_{M}\right)$. Por la Definición 3.1 tenemos que $\varphi_{\gamma * \delta}$ : $R$-pr $\rightarrow T$-pr tal que para $\tau \in R$-pr $\varphi_{\gamma * \delta}(\tau)=\bigvee_{M \in R \text {-Mod }} \alpha_{(\gamma * \delta)_{M} \circ(H \circ F)\left(i_{\tau M}\right)}$, donde $i_{\tau M}: \tau(M) \hookrightarrow M$ y se tiene el siguiente diagrama conmutativo:

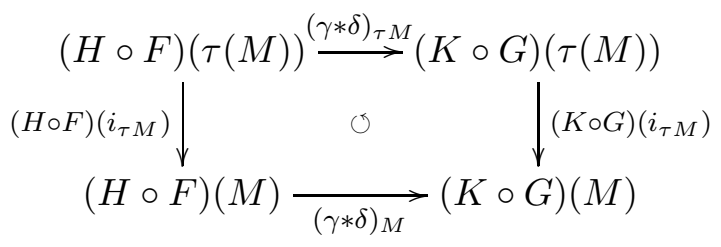


Análogamente, para $\tau \in R$-pr, por la Definición 3.1 se sigue que $\psi_{\gamma * \delta}(\tau)=$ $\bigwedge_{M \in R \text {-Mod }} \omega_{(\gamma * \delta)_{M / \tau M} \circ(H \circ F)\left(p_{\tau M}\right)}$, donde $p_{\tau M}: M \rightarrow M / \tau(M)$ y se tiene el siguiente diagrana conmutativo:

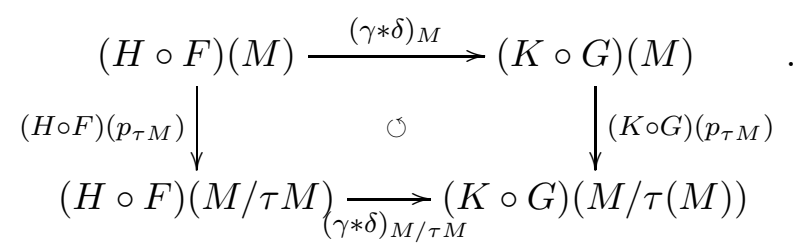

Teorema 3.17. Sean $F: R$-Mod $\rightarrow S$-Mod y $H, K: S$-Mod $\rightarrow T$-Mod funtores tales que $H$ preserva coproductos y epimorfismos. Sea $\gamma: H \rightarrow K$ una transformación natural. Entonces $\varphi_{\gamma * 1_{F}}=\varphi_{\gamma} \circ \varphi_{1_{F}}$.

Demostración. Sea $\tau \in R$-pr. Por la Definición 3.1:

$$
\varphi_{\gamma * 1_{F}}(\tau)=\bigvee_{M \in R \text {-Mod }} \alpha_{\left(\gamma * 1_{F}\right)_{M} \circ(H \circ F)\left(i_{\tau M}\right)},
$$

donde $\left(\gamma * 1_{F}\right)_{M}=\gamma_{F(M)}$ por la Observación 1.30 y se tiene un diagrama como en (41) con $\delta=1_{F}$, donde $i_{\tau M}: \tau(M) \hookrightarrow M$. También de la Definición 3.1:

$$
\begin{aligned}
\left(\varphi_{\gamma} \circ \varphi_{1_{F}}\right)(\tau) & =\varphi_{\gamma}\left(\bigvee_{M \in R \text {-Mod }} \alpha_{1_{F(M)} \circ F\left(i_{\tau M}\right)}\right) \\
& =\varphi_{\gamma}\left(\bigvee_{M \in R \text {-Mod }} \alpha_{F\left(i_{\tau M}\right)}\right)=\bigvee_{N \in S \text {-Mod }} \alpha_{\gamma_{N} \circ H\left(i_{\sigma N}\right)}
\end{aligned}
$$

donde $i_{\sigma N}: \sigma(N) \hookrightarrow N$ y el $S$-prerradical $\sigma=\varphi_{1_{F}}(\tau)=\bigvee_{M \in R \text {-Mod }} \alpha_{F\left(i_{\tau M}\right)}$.

Probaremos primero que $\left(\varphi_{\gamma} \circ \varphi_{1_{F}}\right)(\tau) \preceq \varphi_{\gamma * 1_{F}}(\tau)$. Por lo que basta ver que para toda $N \in S$-Mod, $\alpha_{\gamma_{N} \circ H\left(i_{\sigma N}\right)} \preceq \bigvee_{M \in R \text {-Mod }} \alpha_{\left(\gamma * 1_{F}\right)_{M} \circ(H \circ F)\left(i_{\tau M}\right)}$. Sea $N \in S$ Mod, $L \in T$-Mod y consideremos lo siguiente:

$$
\alpha_{\gamma_{N} \circ H\left(i_{\sigma N}\right)}(L)=\sum\left\{k\left(\operatorname{Im}\left(\gamma_{N} \circ H\left(i_{\sigma N}\right)\right)\right) \mid k: K(N) \rightarrow L\right\}
$$

$\mathrm{y}$

$$
\alpha_{\left(\gamma * 1_{F}\right)_{M} \circ(H \circ F)\left(i_{\tau M}\right)}(L)=\sum\left\{k^{\prime}\left(\operatorname{Im}\left(\gamma_{F(M)} \circ(H \circ F)\left(i_{\tau M}\right)\right) \mid k^{\prime}: K(F(M)) \rightarrow L\right\} .\right.
$$


Sea $\mathcal{H}=\operatorname{Hom}(F(M), N)$. Por el Lema 2.51, existe un epimorfismo $\bar{g}:(F(\tau(M)))^{(\mathcal{H})} \rightarrow \alpha_{F\left(i_{\tau M}\right)}(N)$ tal que el siguiente diagrama es conmutativo para cada $f \in \mathcal{H}$ :

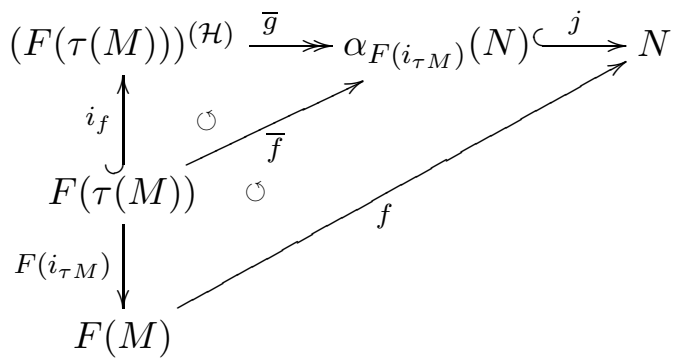

donde $i_{f}$ es la inclusión natural en el coproducto $(F(\tau(M)))^{(\mathcal{H})}, \bar{f}$ es la correstricción del morfismo $f \circ F\left(i_{\tau M}\right)$ a su imagen y $j$ es la inclusión natural. Por la propiedad universal del coproducto:

$$
\operatorname{Im} \bar{g}=\alpha_{F\left(i_{\tau M}\right)}(N)=\sum_{\mathcal{H}} f\left(\operatorname{Im} F\left(i_{\tau M}\right)\right) .
$$

Sea $X_{N}$ un conjunto de $R$-Mod tal que

$$
\sum_{M \in R \text {-Mod }} \alpha_{F\left(i_{\tau M}\right)}(N)=\sum_{M \in X_{N}} \alpha_{F\left(i_{\tau M}\right)}(N) .
$$

Sea $B=(F(\tau(M)))^{(\mathcal{H})}$. Por la propiedad universal del coproducto existe un único homomorfismo $h: B^{\left(X_{N}\right)} \rightarrow \sum_{M \in X_{N}} \alpha_{F\left(i_{\tau M}\right)}(N)$, tal que para cada $M \in X_{N}$ el siguiente diagrama es conmutativo:

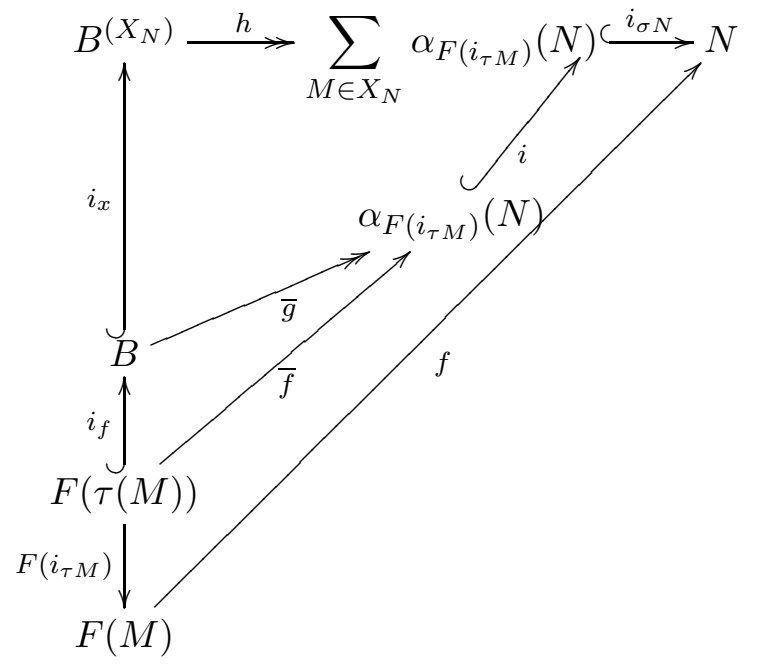

donde $i_{x}$ es la inclusión natural en el coproducto $B^{\left(X_{N}\right)}, i$ es la inclusión natural en $\sum_{M \in X_{N}} \alpha_{F\left(i_{\tau M}\right)}(N)$, e $\operatorname{Im} h=\sum_{M \in X_{N}} \alpha_{F\left(i_{\tau M}\right)}(N)=\sum_{M \in X_{N}} \sum_{\mathcal{H}} f\left(\operatorname{Im} F\left(i_{\tau M}\right)\right)$. 
Sea $k: K(N) \rightarrow L$. Puesto que el funtor $H$ preserva coproductos y epimorfismos, al aplicar el funtor $H$ al diagrama (43) obtenemos el siguiente diagrama conmutativo:

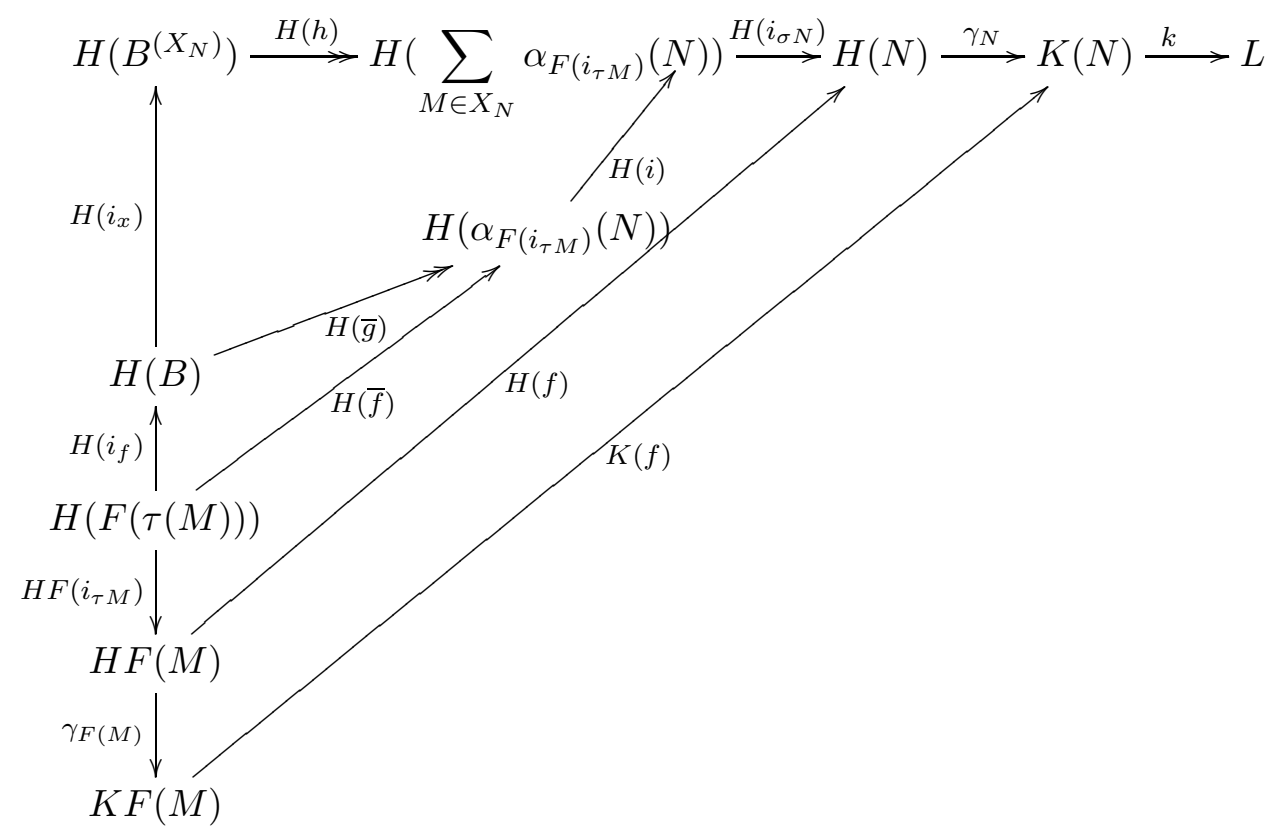

De ahí:

$$
\begin{aligned}
k\left(\operatorname{Im}\left(\gamma_{N} \circ H\left(i_{\sigma_{N}}\right)\right)\right) & =\left(k \circ \gamma_{N} \circ H\left(i_{\sigma N}\right)\right)\left[H\left(\sum_{M \in X_{N}} \alpha_{F\left(i_{\tau M}\right)}(N)\right)\right] \\
& =\left(k \circ \gamma_{N} \circ H\left(i_{\sigma N}\right)\right)\left[\sum_{M \in X_{N}} \operatorname{Im}(H(i) \circ H(\bar{g}))\right] \\
& =\sum_{M \in X_{N}}\left(k \circ \gamma_{N} \circ H\left(i_{\sigma N}\right) \circ H(i)\right)[\operatorname{Im} H(\bar{g})] \\
& =\sum_{M \in X_{N}}\left(k \circ \gamma_{N} \circ H\left(i_{\sigma N}\right) \circ H(i)\right)\left[\sum_{\mathcal{H}} H(\bar{f})\right] \\
& =\sum_{M \in X_{N}} \sum_{\mathcal{H}} \operatorname{Im}\left(k \circ \gamma_{N} \circ H\left(i_{\sigma N}\right) \circ H(i) \circ H(\bar{f})\right) \\
& =\sum_{M \in X_{N}} \sum_{\mathcal{H}} \operatorname{Im}\left(k \circ K(f) \circ \gamma_{F(M)} \circ H F\left(i_{\tau M}\right)\right) \\
& =\sum_{M \in X_{N}} \sum_{\mathcal{H}}(k \circ K(f))\left(\operatorname{Im}\left(\gamma_{F(M)} \circ H F\left(i_{\tau M}\right)\right)\right) \\
& \leq \sum_{M \in X_{N}} \alpha_{\left(\gamma * 1_{F}\right)_{M} \circ(H \circ F)\left(i_{\tau M}\right)}(L) \\
& \leq \bigvee_{M \in R-\operatorname{Mod}} \alpha_{\left(\gamma * 1_{F}\right)_{M} \circ(H \circ F)\left(i_{\tau M}\right)}(L)
\end{aligned}
$$


Por lo tanto $\left(\varphi_{\gamma} \circ \varphi_{1_{F}}\right)(\tau) \preceq \varphi_{\gamma * 1_{F}}(\tau)$.

Ahora sea $\tau \in R$-pr y veamos que $\varphi_{\gamma * 1_{F}}(\tau) \preceq\left(\varphi_{\gamma} \circ \varphi_{1_{F}}\right)(\tau)$. Por lo que probaremos que $\alpha_{\left(\gamma * 1_{F}\right)_{M} \circ(H \circ F)\left(i_{\tau M}\right)} \preceq \bigvee_{N \in S \text {-Mod }} \alpha_{\gamma_{N} \circ H\left(i_{\sigma N}\right)}$ para cada $M \in R$ Mod.

Sea $M_{0} \in R$-Mod y consideremos $N_{0}=F\left(M_{0}\right) \in S$-Mod. Sea $\mathcal{H}=$ $\operatorname{Hom}\left(F\left(M_{0}\right), N_{0}\right)$ y $L \in T$-Mod. Sea $X$ un conjunto en $R$-Mod tal que $M_{0} \in X$ $\mathrm{y}$

$$
\sum_{M \in R \text {-Mod }} \alpha_{F\left(i_{\tau M}\right)}\left(N_{0}\right)=\sum_{M \in X} \alpha_{F\left(i_{\tau M}\right)}\left(N_{0}\right) .
$$

Procediendo de la misma manera que en la prueba de la otra desigualdad, para $k^{\prime}: K\left(F\left(M_{0}\right)\right) \rightarrow L$ se tiene un diagrama similar al anterior que es conmutativo para cada $f \in H$ y en particular para el homomorfismo identidad $f: F\left(M_{0}\right) \rightarrow$ $F\left(M_{0}\right)$. Dicho diagrama es similar al anterior sustituyendo $M$ por $M_{0}, N$ por $N_{0}$, $k$ por $k^{\prime}$ y $X_{N}$ por $X$. Entonces:

$$
\begin{aligned}
\left(k^{\prime} \circ K(f)\right)\left(\operatorname{Im}\left(\gamma_{F\left(M_{0}\right)} \circ H F\left(i_{\tau M_{0}}\right)\right)\right) & =\operatorname{Im}\left(k^{\prime} \circ \gamma_{N_{0}} \circ H\left(i_{\sigma N_{0}}\right) \circ H(i) \circ H(\bar{f})\right) \\
& =k^{\prime}\left(\operatorname{Im}\left(\gamma_{N_{0}} \circ H\left(i_{\sigma N_{0}}\right) \circ H(h) \circ H\left(i_{x}\right) \circ H\left(i_{f}\right)\right)\right) \\
& \leq k^{\prime}\left(\operatorname{Im}\left(\gamma_{N_{0}} \circ H\left(i_{\sigma N_{0}}\right)\right)\right) \leq \alpha_{\gamma_{N_{0}} \circ H\left(i_{\sigma N_{0}}\right)}(L) \\
& \leq \bigvee_{N \in S \text {-Mod }} \alpha_{\gamma_{N} \circ H\left(i_{\sigma N}\right)}(L) .
\end{aligned}
$$

Puesto que $K$ preserva identidades, se tiene que $K(f)=1_{K F\left(M_{0}\right)}$ y así $\left(k^{\prime} \circ\right.$ $K(f))=k^{\prime}$. Por lo tanto

$$
k^{\prime}\left(\operatorname{Im}\left(\gamma_{F\left(M_{0}\right)} \circ H F\left(i_{\tau M_{0}}\right)\right)\right) \leq \bigvee_{N \in S \text {-Mod }} \alpha_{\gamma_{N} \circ H\left(i_{\sigma N}\right)}(L) .
$$

De ahí concluimos que para cada $M \in R$-Mod:

$$
\alpha_{\left(\gamma * 1_{F}\right)_{M} \circ(H \circ F)\left(i_{\tau M}\right)} \preceq \bigvee_{N \in S \text {-Mod }} \alpha_{\gamma_{N} \circ H\left(i_{\sigma N}\right)}
$$

Entonces $\varphi_{\gamma * 1_{F}}(\tau) \preceq\left(\varphi_{\gamma} \circ \varphi_{1_{F}}\right)(\tau)$. Por lo tanto $\varphi_{\gamma * 1_{F}}=\varphi_{\gamma} \circ \varphi_{1_{F}}$.

Teorema 3.18. Sean F $: R$-Mod $\rightarrow S$-Mod y $H, K: S$-Mod $\rightarrow$ T-Mod funtores tales que $K$ preserva productos y monomorfismos. Sea $\gamma: H \rightarrow K$ una transformación natural. Entonces $\psi_{\gamma * 1_{F}}=\psi_{\gamma} \circ \psi_{1_{F}}$.

Demostración. Sea $\tau \in R$-pr. Primero probaremos,

$$
\psi_{\gamma * 1_{F}}(\tau) \preceq\left(\psi_{\gamma} \circ \psi_{1_{F}}\right)(\tau)=\psi_{\gamma}\left(\psi_{1_{F}}(\tau)\right) .
$$

Por la Definición 3.1:

$$
\psi_{\gamma * 1_{F}}(\tau)=\bigwedge_{M \in R \text {-Mod }} \omega_{\left(\gamma * 1_{F}\right)_{M / \tau M} \circ(H \circ F)\left(p_{\tau M}\right)}
$$


y se tiene un diagrama como en (42) con $\delta=1_{F}$, donde $p_{\tau M}: M \rightarrow M / \tau(M)$. Notemos que (44) usando el diagrama (42) es equivalente a:

$$
\begin{aligned}
\psi_{\gamma * 1_{F}}(\tau) & =\bigwedge_{M \in R \text {-Mod }} \omega_{(K \circ F)\left(p_{\tau M}\right) \circ\left(\gamma * 1_{F}\right)_{M}} \\
& =\bigwedge_{M \in R \text {-Mod }} \omega_{(K \circ F)\left(p_{\tau M}\right) \circ(\gamma)_{F(M)}},
\end{aligned}
$$

donde por la Observación 1.30, $\left(\gamma * 1_{F}\right)_{M}=(\gamma)_{F(M)}$.

Sea $\sigma=\psi_{1_{F}}(\tau) \in S$-pr. Entonces de la Definición 3.1 y del diagrama 33:

$$
\begin{aligned}
\psi_{\gamma}\left(\psi_{1_{F}}(\tau)\right) & =\psi_{\gamma}(\sigma)=\bigwedge_{N \in S \text {-Mod }} \omega_{\gamma_{(N / \sigma N)} \circ H\left(p_{\sigma N}\right)} \\
& =\bigwedge_{N \in S-\operatorname{Mod}} \omega_{K\left(p_{\sigma N}\right) \circ \gamma_{N}}
\end{aligned}
$$

donde $p_{\sigma N}: N \rightarrow N / \sigma(N)$ y

$$
\begin{aligned}
\sigma(N) & =\bigwedge_{M \in R \text {-Mod }} \omega_{\left(1_{F}\right)_{M / \tau M} \circ F\left(p_{\tau M}\right)}(N)=\bigwedge_{M \in R \text {-Mod }} \omega_{F(M / \tau M) \circ F\left(p_{\tau M}\right)}(N) \\
& =\bigwedge_{M \in R \text {-Mod }} \omega_{F\left(p_{\tau M}\right)}(N)
\end{aligned}
$$

Entonces para probar que

$$
\bigwedge_{M \in R \text {-Mod }} \omega_{(K \circ F)\left(p_{\tau M}\right) \circ(\gamma)_{F(M)}} \preceq \bigwedge_{N \in S-\operatorname{Mod}} \omega_{K\left(p_{\sigma N}\right) \circ \gamma_{N}}
$$

basta ver que para cada $N \in S$-Mod, se tiene que

$$
\bigwedge_{M \in R \text {-Mod }} \omega_{(K \circ F)\left(p_{\tau M}\right) \circ(\gamma)_{F(M)}} \preceq \omega_{K\left(p_{\sigma N}\right) \circ \gamma_{N}} .
$$

Sea $N \in S$-Mod y $L \in T$-Mod. Entonces:

$$
\begin{gathered}
\omega_{(K \circ F)\left(p_{\tau M}\right) \circ(\gamma)_{F(M)}}(L)=\bigcap\left\{\overleftarrow{k^{\prime}}\left(\operatorname{ker}\left((K \circ F)\left(p_{\tau M}\right) \circ \gamma_{F(M)}\right)\right) \mid k^{\prime}: L \rightarrow H(F(M))\right\} \\
\omega_{K\left(p_{\sigma N}\right) \circ \gamma_{N}}(L)=\bigcap\left\{\overleftarrow{k}\left(\operatorname{ker}\left(K\left(p_{\sigma N}\right) \circ \gamma_{N}\right)\right) \mid k: L \rightarrow H(N)\right\}
\end{gathered}
$$

$\mathrm{y}$

$$
\omega_{F\left(p_{\tau M}\right)}(N)=\bigcap\left\{\overleftarrow{h}\left(\operatorname{ker} F\left(p_{\tau M}\right) \mid h: N \rightarrow F(M)\right\}\right.
$$

Sea $\mathcal{H}=\operatorname{Hom}(N, F(M))$. Por la propiedad universal del producto existe un único homomorfismo $f: N \rightarrow(F(M / \tau M))^{\mathcal{H}}$ tal que el siguiente diagrama es 
conmutativo para cada $h \in \mathcal{H}$ :

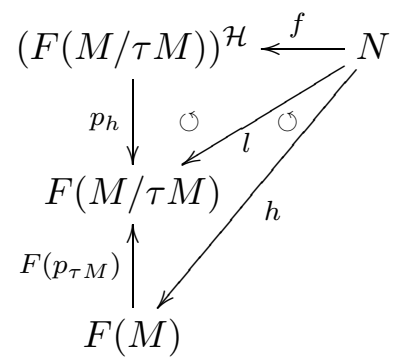

donde $p_{h}$ es la proyección natural del producto $(F(M / \tau M))^{\mathcal{H}}$ y $l=F\left(p_{\tau M}\right) \circ h$. También por la propiedad universal del producto:

$$
\begin{aligned}
\operatorname{ker} f & =\bigcap_{\mathcal{H}} \operatorname{ker} l=\bigcap_{\mathcal{H}} \operatorname{ker}\left(F\left(p_{\tau M}\right) \circ h\right)=\bigcap_{\mathcal{H}} \overleftarrow{h}\left(\operatorname{ker} F\left(p_{\tau M}\right)\right) \\
& =\omega_{F\left(p_{\tau M}\right)}(N)
\end{aligned}
$$

Sea $X_{N}$ un conjunto de $R$-Mod tal que

$$
\bigcap_{M \in R \text {-Mod }} \omega_{F\left(p_{\tau M}\right)}(N)=\bigcap_{M \in X_{N}} \omega_{F\left(p_{\tau M}\right)}(N) .
$$

Sea $B=(F(M / \tau M))^{\mathcal{H}}$. Por la propiedad universal del producto existe un único homomorfismo $f^{\prime}: N \rightarrow B^{X_{N}}$, tal que para cada $M \in X_{N}$ el siguiente diagrama es conmutativo:

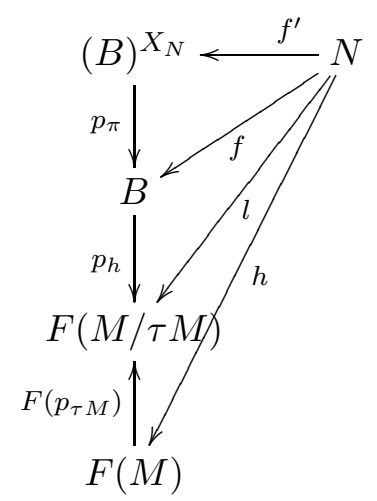

donde $p_{\pi}$ es la proyección natural del producto $B^{X_{N}}$. También de la propiedad universal del producto:

$$
\operatorname{ker} f^{\prime}=\bigcap_{M \in X_{N}} \operatorname{ker} f=\bigcap_{M \in X_{N}} \omega_{F\left(p_{\tau M}\right)}(N)=\sigma(N) .
$$


Sea $k: L \rightarrow H(N)$. Puesto que $N / \sigma(N)=N / \operatorname{ker} f^{\prime} \cong \operatorname{Im} f^{\prime} \hookrightarrow B^{X_{N}}$ y ya que el funtor $K$ preserva productos y monomorfismos, al aplicar el funtor $K$ al diagrama (45) obtenemos el siguiente diagrama conmutativo:

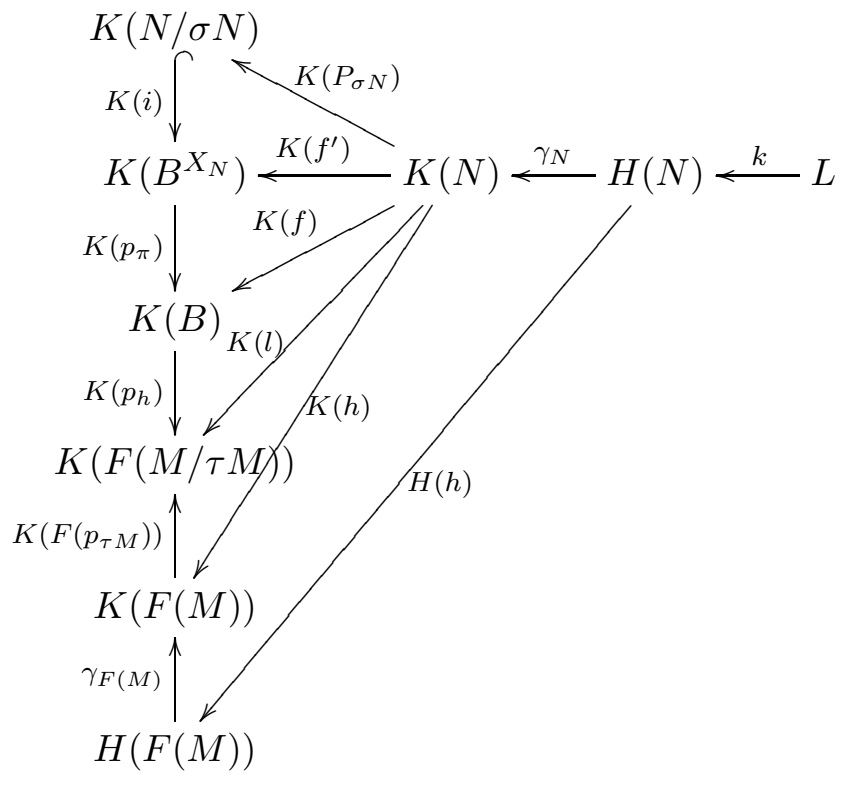

donde $i: N / \sigma(N) \hookrightarrow B^{X_{N}}$. 
De ahí:

$$
\begin{aligned}
& \overleftarrow{k}\left(\operatorname{ker}\left(K\left(p_{\sigma N}\right) \circ \gamma_{N}\right)\right)=\operatorname{ker}\left(K\left(p_{\sigma N}\right) \circ \gamma_{N} \circ k\right) \\
& =\operatorname{ker}\left(K(i) \circ K\left(p_{\sigma N}\right) \circ \gamma_{N} \circ k\right) \\
& =\operatorname{ker}\left(K\left(f^{\prime}\right) \circ \gamma_{N} \circ k\right) \\
& =\overleftarrow{\left(\gamma_{N} \circ k\right)}\left(\operatorname{ker} K\left(f^{\prime}\right)\right) \\
& =\overleftarrow{\left(\gamma_{N} \circ k\right)}\left(\bigcap_{M \in X_{N}} \operatorname{ker} K(f)\right) \\
& =\overleftarrow{\left(\gamma_{N} \circ k\right)}\left(\bigcap_{M \in X_{N}} \bigcap_{\mathcal{H}} \operatorname{ker} K(l)\right) \\
& =\bigcap_{M \in X_{N}} \bigcap_{\mathcal{H}} \operatorname{ker}\left(K(l) \circ \gamma_{N} \circ k\right) \\
& =\bigcap_{M \in X_{N}} \bigcap_{\mathcal{H}} \operatorname{ker}\left(K F\left(p_{\tau M}\right) \circ K(h) \circ \gamma_{N} \circ k\right) \\
& =\bigcap_{M \in X_{N}} \bigcap_{\mathcal{H}} \operatorname{ker}\left(K F\left(p_{\tau M}\right) \circ \gamma_{F(M)} \circ H(h) \circ k\right) \\
& =\bigcap_{M \in X_{N}} \bigcap_{\mathcal{H}} \overleftarrow{(H(h) \circ k)}\left(\operatorname{ker}\left(K F\left(p_{\tau M}\right) \circ \gamma_{F(M)}\right)\right) \\
& \geq \bigcap_{M \in X_{N}} \omega_{(K \circ F)\left(p_{\tau M}\right) \circ \gamma_{F(M)}}(L) \\
& \geq \bigwedge_{M \in R \text {-Mod }} \omega_{(K \circ F)\left(p_{\tau M}\right) \circ \gamma_{F(M)}}(L)
\end{aligned}
$$

De ahí concluimos que para cada $N \in S$-Mod,

$$
\bigwedge_{M \in R \text {-Mod }} \omega_{(K \circ F)\left(p_{\tau M}\right) \circ(\gamma)_{F(M)}}(L) \leq \omega_{K\left(p_{\sigma N}\right) \circ \gamma_{N}}(L) .
$$

Por lo tanto $\psi_{\gamma * 1_{F}}(\tau) \preceq\left(\psi_{\gamma} \circ \psi_{1_{F}}\right)(\tau)$.

Sea $\tau \in R$-pr. Entonces veamos que $\left(\psi_{\gamma} \circ \psi_{1_{F}}\right)(\tau) \preceq \psi_{\gamma * 1_{F}}(\tau)$. Basta ver que para cada $M \in R$-Mod:

$$
\bigwedge_{N \in S \text {-Mod }} \omega_{K\left(p_{\sigma N}\right) \circ \gamma_{N}} \preceq \omega_{(K \circ F)\left(p_{\tau M}\right) \circ(\gamma)_{F(M)}} .
$$

Sea $M_{0} \in R$-Mod y consideremos $N_{0}=F\left(M_{0}\right) \in S$-Mod. Sea $\mathcal{H}=\operatorname{Hom}\left(N_{0}, F\left(M_{0}\right)\right)$ y $L \in T$-Mod. Sea $X$ un conjunto en $R$-Mod tal que $M_{0} \in X$ y

$$
\bigcap_{M \in R \text {-Mod }} \omega_{F\left(p_{\tau M}\right)}\left(N_{0}\right)=\bigcap_{M \in X} \omega_{F\left(p_{\tau M}\right)}\left(N_{0}\right) .
$$

Análogamente a la prueba de la otra desigualdad, para $k^{\prime}: L \rightarrow H\left(F\left(M_{0}\right)\right)$ se tiene un diagrama similar al anterior que es conmutativo para cada $h \in H$ 
y en particular para el homomorfismo identidad $h: F\left(M_{0}\right) \rightarrow F\left(M_{0}\right)$. Dicho diagrama es similar al anterior sustituyendo $M$ por $M_{0}, N$ por $N_{0}, k$ por $k^{\prime} \mathrm{y}$ $X_{N}$ por $X$. Puesto que $H$ preserva identidades tenemos que $H(h)=1_{H F\left(M_{0}\right)}$ y así $\left(H(h) \circ k^{\prime}\right)=k^{\prime}$. Luego:

$$
\begin{aligned}
\overleftarrow{k^{\prime}}\left(\operatorname{ker}\left(K F\left(p_{\tau M_{0}}\right) \circ(\gamma)_{F\left(M_{0}\right)}\right)\right) & =\overleftarrow{\left(H(h) \circ k^{\prime}\right)}\left(\operatorname{ker}\left(K F\left(p_{\tau M_{0}}\right) \circ(\gamma)_{F\left(M_{0}\right)}\right)\right) \\
& =\operatorname{ker}\left(K F\left(p_{\tau M_{0}}\right) \circ \gamma_{F\left(M_{0}\right)} \circ H(h) \circ k^{\prime}\right) \\
& =\operatorname{ker}\left(K(l) \circ \gamma_{N_{0}} \circ k^{\prime}\right) \\
& =\operatorname{ker}\left(K\left(p_{h}\right) \circ K\left(p_{\pi}\right) \circ K(i) \circ K\left(p_{\sigma N_{0}}\right) \circ \gamma_{N_{0}} \circ k^{\prime}\right) \\
& \geq \operatorname{ker}\left(K\left(p_{\sigma N_{0}}\right) \circ \gamma_{N_{0}} \circ k^{\prime}\right) \\
& =\overleftarrow{k^{\prime}}\left(\operatorname{ker}\left(K\left(p_{\sigma_{N}}\right) \circ \gamma_{N_{0}}\right)\right) \\
& \geq \omega_{K\left(p_{\sigma N_{0}}\right) \circ \gamma_{N_{0}}}(L) \\
& \geq \bigwedge_{N \in S \text {-Mod }} \omega_{K\left(p_{\sigma N}\right) \circ \gamma_{N}}(L)
\end{aligned}
$$

De donde concluimos que para cada $M \in R$-Mod:

$$
\bigwedge_{N \in S \text {-Mod }} \omega_{K\left(p_{\sigma N}\right) \circ \gamma_{N}}(L) \leq \omega_{(K \circ F)\left(p_{\tau M}\right) \circ(\gamma)_{F(M)}}(L) .
$$

Entonces $\left(\psi_{\gamma} \circ \psi_{1_{F}}\right)(\tau) \preceq \psi_{\gamma * 1_{F}}(\tau)$ y de las dos desigualdades llegamos al resultado.

\subsection{Morfismos asociados a funtores}

En la sección 3.1, definimos los morfismos de clapos $\varphi_{\gamma}$ y $\psi_{\gamma}$ con $\gamma: F \rightarrow G$ una transformación natural. En esta sección trabajaremos con el caso particular en el que la transforación natural es la identidad sobre un funtor en la categoría $R$-Mod.

Sea $F: R$-Mod $\rightarrow S$-Mod un funtor y $\gamma=1_{F}: F \rightarrow F$ la transformación natural identidad sobre $F$. Entonces, para $\tau \in R$-pr por la Definición 3.1:

1 .

$$
\begin{aligned}
\varphi_{\gamma}(\tau) & =\bigvee_{M \in R-\operatorname{Mod}} \alpha_{\gamma_{M} \circ F\left(i_{\tau M}\right)}=\bigvee_{M \in R \text {-Mod }} \alpha_{1_{F(M)} \circ F\left(i_{\tau M}\right)} \\
& =\bigvee_{M \in R \text {-Mod }} \alpha_{F\left(i_{\tau M}\right)}=\varphi_{F}(\tau)
\end{aligned}
$$

donde $i_{\tau M}: \tau(M) \hookrightarrow M$ es la inclusión natural.

2.

$$
\begin{aligned}
\psi_{\gamma}(\tau) & =\bigwedge_{M \in R \text {-Mod }} \omega_{\gamma_{(M / \tau M)} \circ F\left(p_{\tau M}\right)}=\bigwedge_{M \in R-\operatorname{Mod}} \omega_{1_{F(M / \tau M)} \circ F\left(p_{\tau M}\right)} \\
& =\bigwedge_{M \in R \text {-Mod }} \omega_{F\left(p_{\tau M}\right)}=\psi_{F}(\tau)
\end{aligned}
$$

donde $p_{\tau M}: M \rightarrow M / \tau(M)$ es la proyección natural. 
Observación 3.19. Sea $F: R$-Mod $\rightarrow$ S-Mod un funtor y $\gamma=1_{F}: F \rightarrow F$ la transformación natural identidad sobre $F$. Entonces denotaremos a $\varphi_{1_{F}}$ y $\psi_{1_{F}}$ respectivamente como:

$$
\varphi_{F} \quad y \quad \psi_{F} .
$$

Observación 3.20. Del Teorema 2.46 se tiene que una adjunción $\langle F, G\rangle$, donde $F: R$-Mod $\rightarrow S$-Mod y $G: S$-Mod $\rightarrow R$-Mod, induce una conexión de Galois $\langle R$-pr, $\varphi, \psi, S$-pr $\rangle$. Por el Teorema 2.54, se tiene que para $\tau \in R$-pr y $\sigma \in S$-pr:

$$
\varphi(\tau)=\bigvee_{M \in R-M o d} \alpha_{F\left(i_{\tau M}\right)} \quad y \quad \psi(\sigma)=\bigwedge_{N \in S-M o d} \omega_{G\left(p_{\sigma N}\right)}
$$

Por otro lado, por la Observación 3.19 para los funtores $F$ y $G$ se tienen los morfismos $\varphi_{F}$ y $\psi_{G}$. Así que notemos lo siguiente para $\tau \in R$-pr y $\sigma \in S$-pr:

1. $\varphi_{F}(\tau)=\bigvee_{M \in R-M o d} \alpha_{F\left(i_{\tau M}\right)}=\varphi(\tau)$

2. $\psi_{G}(\sigma)=\bigwedge_{N \in S-M o d} \omega_{G\left(p_{\sigma N}\right)}=\psi(\sigma)$.

Es decir, la definición de los morfismos $\varphi_{F}$ y $\psi_{G}$ coincide con la definición que teníamos de $\varphi$ y $\psi$ cuando $F$ es un funtor adjunto izquierdo de $G$, o bien, por la Observación 2.55 cuando $F$ y $G$ son funtores equivalentes.

Corolario 3.21. Sean F, G:R-Mod $\rightarrow$ S-Mod funtores y $\gamma: F \rightarrow G$ una transformación natural. Entonces para $\tau \in R$-pr:

1. $\varphi_{\gamma}(\tau) \preceq \varphi_{F}(\tau), \varphi_{\gamma}(\tau) \preceq \varphi_{G}(\tau)$

2. $\psi_{\gamma}(\tau) \succeq \psi_{F}(\tau), \psi_{\gamma}(\tau) \succeq \psi_{G}(\tau)$.

Demostración. 1. Sea $\delta=1_{G}: G \rightarrow G$ la transformación natural identidad sobre $G$ y $\tau \in R$-pr. Por la Proposición 3.3 y la Observación 3.19,obtenemos $\varphi_{\gamma}(\tau) \preceq \varphi_{G}(\tau)$, puesto que:

$$
\varphi_{\gamma}(\tau)=\varphi_{\delta \gamma}(\tau) \preceq \varphi_{\delta}(\tau)=\varphi_{G}(\tau) .
$$

Ahora, sea $\delta=1_{F}: F \rightarrow F$ la transformación natural identidad sobre $F$. Análogamente a lo anterior tenemos:

$$
\varphi_{\gamma}(\tau)=\varphi_{\gamma \delta}(\tau) \preceq \varphi_{\delta}(\tau)=\varphi_{F} .
$$

2. La prueba de la parte 2 es dual a la de 1 .

Corolario 3.22. Sean F, $G: R$-Mod $\rightarrow S$-Mod funtores $y \gamma: F \rightarrow G$ un isomorfismo natural. Entonces

1. $\varphi_{\gamma}=\varphi_{F}=\varphi_{G}$

2. $\psi_{\gamma}=\psi_{F}=\psi_{G}$. 
Demostración. 1. Sea $\delta=1_{G}: G \rightarrow G$ la transformación natural identidad sobre $G$. Por el Corolario 3.5, tenemos $\varphi_{\gamma}=\varphi_{G}$, puesto que:

$$
\varphi_{\gamma}=\varphi_{\delta \gamma}=\varphi_{\delta}=\varphi_{G}
$$

También, si ahora $\delta=1_{F}: F \rightarrow F$ es la transformación natural identidad sobre $F$, obtenemos:

$$
\varphi_{\gamma}=\varphi_{\gamma \delta}=\varphi_{\delta}=\varphi_{F} .
$$

Dualmente a lo anterior y usando el Corolario 3.6, se tiene la parte 2.

Del Corolario 3.22 es inmediato que los morfismos $\varphi_{F}$ y $\psi_{F}$ son los mismos para todos los funtores isomorfos naturalmente a $F$.

Corolario 3.23. Sean $F, F^{\prime}: R$-Mod $\rightarrow S$-Mod funtores tales que $F \cong F^{\prime}$. Entonces

1. $\varphi_{F}=\varphi_{F^{\prime}}$.

2. $\psi_{F}=\psi_{F^{\prime}}$.

Corolario 3.24. Sea $1_{R \text {-Mod }}: R$-Mod $\rightarrow R$-Mod el funtor identidad, $0_{R-M o d}:$ $R$-Mod $\rightarrow S$-Mod el funtor cero y $1_{R-p r}: R$-pr $\rightarrow R$-pr el morfismo identidad en R-pr. Entonces:

1. $\varphi_{1_{R-M o d}}=1_{R-p r}$.

2. $\psi_{1_{R-M o d}}=1_{R-p r}$

3. $\varphi_{0_{R-M o d}}=\mathbf{0}_{R-p r}$, donde $\mathbf{0}_{R-p r}: R-p r \rightarrow R$-pr es el morfismo constante cero.

4. $\psi_{0_{R-M o d}}=\mathbf{1}_{R-p r}$, donde $\mathbf{1}_{R-p r}: R-p r \rightarrow R$-pr es el morfismo constante uno.

Demostración. Sea $\tau \in R$-pr. Entonces:

$$
\begin{aligned}
& \text { 1. } \varphi_{1_{R \text {-Mod }}}(\tau)=\bigvee_{M \in R \text {-Mod }} \alpha_{1_{R \text {-Mod }}\left(i_{\tau M}\right)}=\bigvee_{M \in R \text {-Mod }} \alpha_{i_{\tau M}}=\bigvee_{M \in R \text {-Mod }} \alpha_{\tau M}^{M}= \\
& \tau \text {. } \\
& \text { 2. } \psi_{1_{R-\mathrm{Mod}}}(\tau)=\bigwedge_{M \in R \text {-Mod }} \omega_{1_{R-\mathrm{Mod}}\left(p_{\tau M}\right)}=\bigwedge_{M \in R \text {-Mod }} \omega_{p_{\tau M}}=\bigwedge_{M \in R \text {-Mod }} \omega_{\tau M}^{M}=
\end{aligned}
$$
$\tau$.

3. $\varphi_{0_{R \text {-Mod }}}(\tau)=\bigvee_{M \in R \text {-Mod }} \alpha_{0_{R \text {-Mod }}\left(i_{\tau M}\right)}=\bigvee_{M \in R \text {-Mod }} \alpha_{0}^{0}=0_{R}$, donde $0_{R}$ representa al elemento menor en $R$-pr.

4. $\psi_{0_{R \text {-Mod }}}(\tau)=\bigwedge_{M \in R \text {-Mod }} \omega_{0_{R-\operatorname{Mod}}\left(p_{\tau M}\right)}=\bigwedge_{M \in R \text {-Mod }} \omega_{0}^{0}=1_{R}$, donde $1_{R}$ representa al elemento mayor en $R$-pr.

Corolario 3.25. Sea F $: R$-Mod $\rightarrow S$-Mod una equivalencia. Entonces:

1. $\varphi_{G F}=\psi_{G F}=1_{R-p r}$.

2. $\varphi_{F G}=\psi_{F G}=1_{S-p r}$. 
Donde el funtor $G: S$-Mod $\rightarrow R$-Mod cumple el Teorema 1.32, $1_{R-p r}, 1_{S-p r}$ son el morfismo identidad en $R$-pr y $S$-pr respectivamente.

Demostración. Puesto que $F: R$-Mod $\rightarrow S$-Mod es una equivalencia, existe

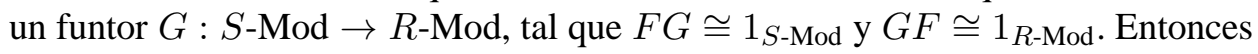
por la Proposición 3.23 y el Corolario 3.24:

1. $\varphi_{G F}=\varphi_{1_{R \text {-Mod }}}=1_{R \text {-pr }}$. También $\psi_{G F}=\psi_{1_{R \text {-Mod }}}=1_{R \text {-pr }}$.

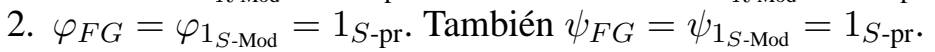

El siguiente resultado es consecuencia inmediata del Corolario 3.7.

Corolario 3.26. Sea $F: R-M o d \rightarrow S-M o d$ un funtor. Entonces:

1. $\varphi_{F}\left(1_{R}\right)=\bigvee_{M \in R-M o d} \alpha_{F(M)}^{F(M)}$, es decir, $\varphi_{F}\left(1_{R}\right)$ es un prerradical idempotente.

2. $\varphi_{F}\left(0_{R}\right)=\bigvee_{M \in R-M o d} \alpha_{I m F\left(i_{0_{R} M}\right)}^{F(M)}$.

3. $\psi_{F}\left(1_{R}\right)=\bigwedge_{M \in R-M o d} \omega_{\operatorname{ker} F\left(p_{M}\right)}^{F(M)}$, donde $p_{M}: M \rightarrow 0$.

4. $\psi_{F}\left(0_{R}\right)=\bigwedge_{M \in R \text {-Mod }} \omega_{0}^{F(M)}$, es decir, $\psi_{F}\left(0_{R}\right)$ es un radical.

Demostración. Se sigue del Corolario 3.7 con $\gamma=1_{F}$ la transformación natural identidad sobre $F$.

Observación 3.27. Nótese que se tiene un comentario similar al de la Observación 3.8 al considerar $\gamma_{1_{F}}$ en el Corolario 3.26 .

Considerando $\gamma=1_{F}$ la transformación natural identidad sobre el funtor $F$ en (38), tenemos a los prerradicales $\widehat{\varepsilon}_{F}=\varphi_{F}\left(1_{R}\right)=\bigvee_{M \in R \text {-Mod }} \alpha_{F(M)}^{F(M)}$ y $\widehat{\eta}_{F}=$ $\psi_{F}\left(0_{R}\right)=\bigwedge_{M \in R-\operatorname{Mod}} \omega_{0}^{F(M)}$

Corolario 3.28. Sean $F, F^{\prime}: R$-Mod $\rightarrow S$-Mod funtores tales que $F \cong F^{\prime}$. Entonces $\widehat{\varepsilon}_{F}=\widehat{\varepsilon}_{F^{\prime}}$ y $\widehat{\eta}_{F}=\widehat{\eta}_{F^{\prime}}$.

Demostración. El resultado es inmediato del Corolario 3.23.

Los siguientes resultados son consecuencia de los Teoremas 3.17 y 3.18 .

Teorema 3.29. Sean $F: R$-Mod $\rightarrow S$-Mod, $G: S$-Mod $\rightarrow T$-Mod funtores tales que $G$ preserva coproductos y epimorfismos. Entonces $\varphi_{G F}=\varphi_{G} \circ \varphi_{F}$. 
Demostración. Sea $\gamma=1_{G}$ la transformación natural identidad sobre el funtor $G$. Notemos que para cada $M \in R$-Mod por la Observación 1.30:

$$
\left(\gamma * 1_{F}\right)_{M}=\left(1_{G} * 1_{F}\right)_{M}=\left(1_{G}\right)_{F(M)}=1_{G(F(M))}=\left(1_{G \circ F}\right)_{M} .
$$

Entonces por el Teorema 3.17 obtenemos el resultado:

$$
\varphi_{G F}=\varphi_{1_{G \circ F}}=\varphi_{\gamma * 1_{F}}=\varphi_{\gamma} \circ \varphi_{1_{F}}=\varphi_{G} \circ \varphi_{F} .
$$

Teorema 3.30. Sean F $: R$-Mod $\rightarrow S$-Mod, $G: S$-Mod $\rightarrow T$-Mod funtores tales que $G$ preserva productos y monomorfismos. Entonces $\psi_{G F}=\psi_{G} \circ \psi_{F}$.

Demostración. El resultado es inmediato del Teorema 3.18, considerando $\gamma=$ $1_{G}$.

Observación 3.31. Notemos que si F $: R$-Mod $\rightarrow$ S-Mod y $G: S$-Mod $\rightarrow R$-Mod son funtores equivalentes, entonces $\varphi_{G}=\psi_{G} y \varphi_{F}=\psi_{F}$. Lo cual se tiene del Corolario 3.25 y los Teoremas 3.29 y 3.30, ya que

$$
\varphi_{G} \circ \varphi_{F}=\varphi_{G F}=1_{R-p r}=\psi_{G F}=\psi_{G} \circ \psi_{F} .
$$

Cabe mencionar que del Teorema 2.22 y la Observación 3.20 también se deduce el resultado ya que en ese caso, $\psi_{G} \circ \varphi_{F}=1_{R-p r}$.

Los siguientes teoremas son una generalización de la Proposiciones 2.52 y 2.53 .

Teorema 3.32. Sean F, $G: R$-Mod $\rightarrow S$-Mod funtores tales que $F$ preserva coproductos y epimorfismos. Sea $\gamma: F \rightarrow G$ una transformación natural y $h$ : $N \rightarrow M$ un morfismo en R-Mod. Entonces $\varphi_{\gamma}\left(\alpha_{h}\right)=\alpha_{\gamma_{M} \circ F(h)}$.

Demostración. Primero probaremos que $\varphi_{\gamma}\left(\alpha_{h}\right) \preceq \alpha_{\gamma_{M} \circ F(h)}$. Entonces por la Definición 3.1:

$$
\varphi_{\gamma}\left(\alpha_{h}\right)=\bigvee_{M \in R-\operatorname{Mod}} \alpha_{\gamma_{M} \circ F\left(i_{\alpha_{h} M}\right)}
$$

donde $i_{\alpha_{h} M}: \alpha_{h}(M) \hookrightarrow M$ y para $M \in R$-Mod

$$
\alpha_{h}(M)=\sum\{f(\operatorname{Im} h) \mid f: M \rightarrow M\}
$$

Entonces para probar que $\bigvee_{M \in R \text {-Mod }} \alpha_{\gamma_{M} \circ F\left(i_{\alpha_{h} M}\right)} \preceq \alpha_{\gamma_{M} \circ F(h)}$, veremos que para cada $M \in R$-Mod

$$
\alpha_{\gamma_{M} \circ F\left(i_{\alpha_{h} M}\right)} \preceq \alpha_{\gamma_{M} \circ F(h)} .
$$

Sean $M \in R$-Mod y $K \in S$-Mod. Se tiene que

$$
\alpha_{\gamma_{M} \circ F(h)}(K)=\sum\left\{g\left(\operatorname{Im}\left(\gamma_{M} \circ F(h)\right)\right) \mid g: G(M) \rightarrow K\right\}
$$


$\mathrm{y}$

$$
\alpha_{\gamma_{M} \circ F\left(i_{\alpha_{h} M}\right)}(K)=\sum\left\{g^{\prime}\left(\operatorname{Im}\left(\gamma_{M} \circ F\left(i_{\alpha_{h} M}\right)\right)\right) \mid g^{\prime}: G(M) \rightarrow K\right\}
$$

Sea $H=\operatorname{Hom}(M, M)$. Por el Lema 2.51, existe un epimorfismo $\bar{g}: N^{(H)} \rightarrow$ $\alpha_{h}(M)$ tal que el siguiente diagrama es conmutativo para cada $f \in H$ :

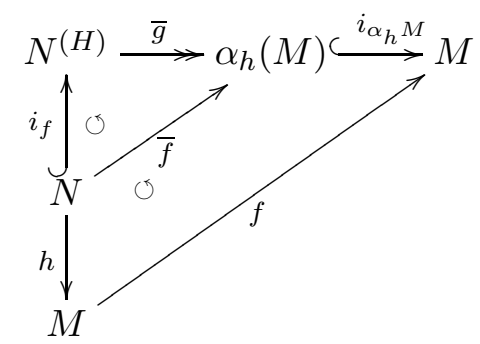

donde $i_{f}$ es la inclusión natural en el coproducto $N^{(H)}, \bar{f}$ es la correstricción del morfismo $f \circ h$ a su imagen. Por la propiedad universal del coproducto:

$$
\operatorname{Im} \bar{g}=\alpha_{h}(N)=\sum_{H} f(\operatorname{Im} h)
$$

Sea $g^{\prime}: G(M) \rightarrow K$. Puesto que el funtor $F$ preserva coproductos y epimorfismos, al aplicar el funtor $F$ al diagrama anterior obtenemos el siguiente diagrama conmutativo:

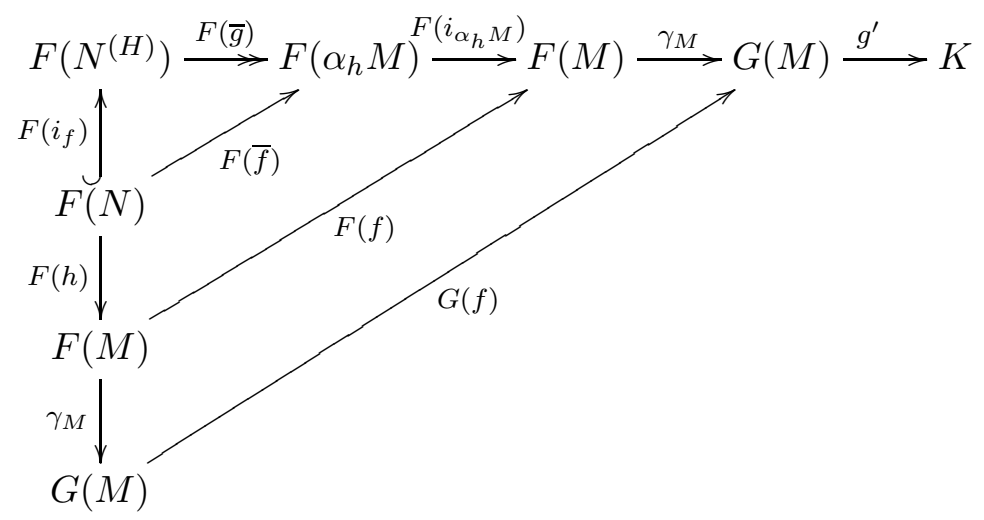


De ahí:

$$
\begin{aligned}
g^{\prime}\left(\operatorname{Im}\left(\gamma_{M} \circ F\left(i_{\alpha_{h} M}\right)\right)\right) & =\left(g^{\prime} \circ \gamma_{M} \circ F\left(i_{\alpha_{h} M}\right)\left[F\left(\alpha_{h} M\right)\right]\right. \\
& =\left(g^{\prime} \circ \gamma_{M} \circ F\left(i_{\alpha_{h} M}\right)\left[\sum_{H} \operatorname{Im}(F(\bar{f}))\right]\right. \\
& =\sum_{H} \operatorname{Im}\left(g^{\prime} \circ \gamma_{M} \circ F\left(i_{\alpha_{h} M} \circ F(\bar{f})\right)\right. \\
& =\sum_{H} \operatorname{Im}\left(g^{\prime} \circ G(f) \circ \gamma_{M} \circ F(h)\right) \\
& =\sum_{H}\left(g^{\prime} \circ G(f)\right)\left(\operatorname{Im}\left(\gamma_{M} \circ F(h)\right)\right) \\
& \leq \alpha_{\gamma_{M} \circ F(h)}(N)
\end{aligned}
$$

Por lo que para cada $M \in R$-Mod, $\alpha_{\gamma_{M} \circ F\left(i_{\alpha_{h} M}\right)} \preceq \alpha_{\gamma_{M} \circ F(h)}$. Entonces $\varphi_{\gamma}\left(\alpha_{h}\right) \preceq$ $\alpha_{\gamma_{M} \circ F(h)}$

Por otro lado, para $M \in R$-Mod, $N \in S$-Mod y $g: G(M) \rightarrow K$, tenemos un Diagrama similar a (46) con $g$ en lugar de $g^{\prime}$, el cual es conmutativo para cada $f \in H$ y en particular para el morfismo identidad $f: M \rightarrow M$. Entonces

$$
\begin{aligned}
g\left(\operatorname{Im}\left(\gamma_{M} \circ F(h)\right)\right) & =\operatorname{Im}\left(g \circ 1_{G(M)} \circ \gamma_{M} \circ F(h)\right) \\
& =\operatorname{Im}\left(g \circ \gamma_{M} \circ F\left(i_{\alpha_{h} M}\right) \circ F(\bar{f})\right) \\
& \leq \operatorname{Im}\left(g \circ \gamma_{M} \circ F\left(i_{\alpha_{h} M}\right)\right) \\
& \leq \alpha_{\gamma_{M} \circ F\left(i_{\alpha_{h} M}\right)}(N)
\end{aligned}
$$

De donde,

$$
\alpha_{\gamma_{M} \circ F(h)} \preceq \alpha_{\gamma_{M} \circ F\left(i_{\alpha_{h} M}\right)} \preceq \bigvee_{M \in R \text {-Mod }} \alpha_{\gamma_{M} \circ F\left(i_{\alpha_{h} M}\right)} .
$$

Por lo tanto, $\alpha_{\gamma_{M} \circ F(h)} \preceq \varphi_{\gamma}\left(\alpha_{h}\right)$ y concluimos la igualdad.

Teorema 3.33. Sean F, $G: R$-Mod $\rightarrow S$-Mod funtores tales que $G$ preserva productos y monomorfismos. Sea $\gamma: F \rightarrow G$ una transformación natural y $h$ : $M \rightarrow N$ un morfismo en R-Mod. Entonces $\psi_{\gamma}\left(\omega_{h}\right)=\omega_{G(h) \circ \gamma_{M}}$.

Demostración. Probaremos primero que $\psi_{\gamma}\left(\omega_{h}\right) \succeq \omega_{G(h) \circ \gamma_{M}}$. Por el diagrama (33) y la Definición 3.1, $\psi_{\gamma}\left(\omega_{h}\right)=\bigwedge_{M \in R \text {-Mod }} \omega_{G\left(p_{\tau M}\right) \circ \gamma_{M}}$, donde $\tau=\omega_{h} \mathrm{y} \tau_{M}$ : $M \rightarrow M / \tau(M)$.

Sea $M \in R$-Mod y veamos que $\omega_{G(h) \circ \gamma_{M}} \preceq \omega_{G\left(p_{\tau M}\right) \circ \gamma_{M}}$. Sea $K \in S$-Mod. Entonces:

$$
\begin{aligned}
\omega_{G\left(p_{\tau M}\right) \circ \gamma_{M}}(K) & =\bigcap\left\{\overleftarrow{g}\left(\operatorname{ker}\left(G\left(p_{\tau M}\right) \circ \gamma_{M}\right)\right) \mid g: K \rightarrow F(M)\right\} \\
\omega_{G(h) \circ \gamma_{M}}(K) & =\bigcap\left\{\overleftarrow{g}\left(\operatorname{ker}\left(G(h) \circ \gamma_{M}\right)\right) \mid g: K \rightarrow F(M)\right\} \\
& =\bigcap\left\{\overleftarrow{g}\left(\operatorname{ker}\left(\gamma_{N} \circ F(h)\right)\right) \mid g: K \rightarrow F(M)\right\}
\end{aligned}
$$


donde la última igualdad es por la naturalidad de $\gamma$ en el morfismo $h \mathrm{y}$

$$
\tau(M)=\bigcap\{\overleftarrow{f}(\operatorname{ker} h) \mid f: M \rightarrow M\}
$$

Sea $H=\operatorname{Hom}(M, M)$. Por la propiedad universal del producto existe un único $l: M \rightarrow(M / \operatorname{ker} h)^{H}$ tal que el siguiente diagrama es conmutativo para cada $f \in H:$

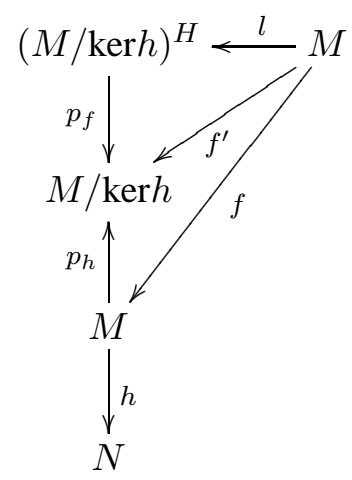

donde $p_{f}$ es la proyección natural del producto $(M / \operatorname{ker} h)^{H}$ y $f^{\prime}=p_{h} \circ f$. También por la propiedad universal del producto:

$$
\begin{aligned}
\operatorname{kerl} & =\bigcap_{H} \operatorname{ker} f^{\prime}=\bigcap_{H} \operatorname{ker}\left(p_{h} \circ f\right)=\bigcap_{H} \overleftarrow{f}(\operatorname{ker} h) \\
& =\tau(M)
\end{aligned}
$$

Sea $g: K \rightarrow F(M)$. Puesto que $M / \tau(M)=M / \operatorname{ker} l \cong \operatorname{Im} l \hookrightarrow(M / \operatorname{ker} h)^{H}$ y ya que el funtor $G$ preserva productos y monomorfismos, al aplicar dicho funtor al diagrama (47) obtenemos el siguiente diagrama conmutativo:

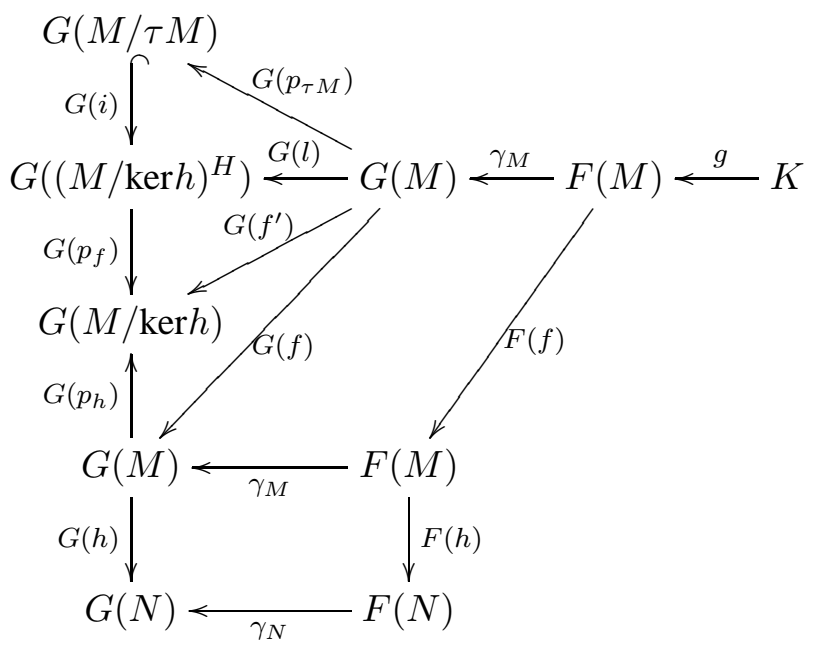


donde $i: M / \tau(M) \hookrightarrow(M / \operatorname{ker} h)^{H}$. Se tiene que $\operatorname{ker} G\left(p_{h}\right)=\operatorname{ker} G(h)$, así que:

$$
\begin{aligned}
\overleftarrow{g}\left(\operatorname{ker}\left(G\left(p_{\tau M}\right) \circ \gamma_{M}\right)\right) & =\operatorname{ker}\left(G(i) \circ G\left(p_{\tau M}\right) \circ \gamma_{M} \circ g\right) \\
& =\overleftarrow{\left(\gamma_{M} \circ g\right)}(\operatorname{ker} G(l)) \\
& =\overleftarrow{\left(\gamma_{M} \circ g\right)}\left(\bigcap_{H} \operatorname{ker}\left(G\left(p_{h}\right) \circ G(f)\right)\right) \\
& =\overleftarrow{\left(G(F) \circ \gamma_{M} \circ g\right)}\left(\bigcap_{H} \operatorname{ker} G\left(p_{h}\right)\right) \\
& =\overleftarrow{\left(G(F) \circ \gamma_{M} \circ g\right)}\left(\bigcap_{H} \operatorname{ker} G(h)\right) \\
& =\bigcap_{H}\left(\gamma_{N} \circ F(h) \circ F(f) \circ g\right) \\
& =\bigcap_{H} \overleftarrow{(F(f) \circ g)}\left(\operatorname{ker}\left(\gamma_{N} \circ F(h)\right)\right. \\
& \supseteq \omega_{G(h) \circ \gamma_{M}}(K)
\end{aligned}
$$

Por lo tanto, $\omega_{G\left(p_{\tau M}\right) \circ \gamma_{M}}(K) \geq \omega_{G(h) \circ \gamma_{M}}(K)$, de donde se concluye que $\psi_{\gamma}\left(\omega_{h}\right) \succeq$ $\omega_{G(h) \circ \gamma_{M}}$.

La prueba de la otra desigualdad es similar y dual a la prueba de la segunda parte del Teorema 3.32. Entonces $\psi_{\gamma}\left(\omega_{h}\right)=\omega_{G(h) \circ \gamma_{M}}$.

Los siguientes corolarios son inmediatos de los Teoremas 3.32 y 3.33 respectivamente, considerando $\gamma=1_{F}$.

Corolario 3.34. Sea $h: N \rightarrow M$ un morfismo en $R$-Mod, $F: R$-Mod $\rightarrow S$-Mod un funtor que preserva coproductos y epimorfismos. Entonces $\varphi_{F}\left(\alpha_{h}\right)=\alpha_{F(h)}$.

Corolario 3.35. Sea $h: M \rightarrow N$ un morfismo en $R$-Mod, $F: R$-Mod $\rightarrow S$-Mod un funtor que preserva productos y monomorfismos. Entonces $\psi_{F}\left(\omega_{h}\right)=\omega_{F(h)}$.

\subsection{Morfismos asociados a prerradicales y coprerradica- les}

En esta sección estudiaremos los morfismos $\varphi_{F}$ y $\psi_{F}$ vistos en la sección anterior para el caso en el que $F$ es un prerradical o un coprerradical. Algunos resultados que presentamos al final de esta sección son la obtención de los operadores de la Definición 1.81, ver [9], como imágenes de ciertos prerradicales bajo los morfismos $\varphi_{F} \mathrm{O} \psi_{F}$.

\subsubsection{Obtención del Igualador}

Proposición 3.36. Sean $\sigma, \tau \in R$-pr. Entonces $\sigma \tau \preceq \varphi_{\sigma}(\tau)$. 
Demostración. Sean $\sigma, \tau \in R$-pr. Por la Proposición 3.12, $\varphi_{i_{\sigma, \sigma}}(\tau) \succeq \sigma \tau$. Entonces

$$
\varphi_{\sigma}(\tau)=\varphi_{1_{\sigma}}(\tau)=\varphi_{i_{\sigma, \sigma}}(\tau) \succeq \sigma \tau
$$

Observación 3.37. Nótese que si $\sigma \tau=\tau \sigma$, entonces $\varphi_{\sigma}(\tau) \preceq \tau$. Puesto que

$$
\begin{aligned}
\varphi_{\sigma}(\tau)= & \bigvee_{M \in R \text {-Mod }} \alpha_{\sigma\left(i_{\tau M}\right)} \text { y para } K \in R \text {-Mod, tenemos } \\
\alpha_{\sigma\left(i_{\tau M}\right)}(K) & =\sum\left\{f\left(\operatorname{Im} \sigma\left(i_{\tau M}\right)\right) \mid f: \sigma(M) \rightarrow K\right\} \\
& =\sum\{f(\sigma(\tau(M)))=f(\tau(\sigma(M))) \mid f: \sigma(M) \rightarrow K\}
\end{aligned}
$$

donde $f(\tau(\sigma(M))) \leq \tau(K)$, por ser $\tau$ un R-prerradical.

Lema 3.38. Sea $\sigma \in R$-pr. Entonces $e(\sigma)=\varphi_{\sigma}\left(1_{R}\right)$.

Demostración. Es inmediato de la parte 1 del Corolario 3.26 y del inciso c) del Teorema 1.82 .

Lema 3.39. Sea $\sigma \in R$-pr. Entonces $\sigma$ es un prerradical idempotente si y sólo si $\varphi_{\sigma}(\tau) \preceq \sigma$ para toda $\tau \in R-p r$.

Demostración. Sea $\tau \in R$-pr. Entonces $\varphi_{\sigma}(\tau)=\bigvee_{M \in R \text {-Mod }} \alpha_{\sigma\left(i_{\tau M}\right)}$. Supongamos que $\sigma$ es un prerradical idempotente. Sea $K \in R$-Mod, así

$$
\alpha_{\sigma\left(i_{\tau M}\right)}(K)=\sum\{f(\sigma(\tau(M))) \mid f: \sigma(M) \rightarrow K\} .
$$

Para cada $f: \sigma(M) \rightarrow K$ por ser $\sigma$ un prerradical idempotente tenemos que $f(\sigma(M))=f(\sigma(\sigma(M))) \leq \sigma(K)$. Puesto que $\sigma(\tau(M)) \leq \sigma(M)$ y de lo anterior obtenemos que $f(\sigma(\tau(M))) \leq \sigma(K)$. De donde, $\varphi_{\sigma}(\tau) \preceq \sigma$.

Por otro lado, supongamos que $\varphi_{\sigma}(\tau) \preceq \sigma$ para toda $\tau \in R$-pr. Por lo que considerando $\tau=1_{R}$, por el inciso a) del Teorema 1.82 y el Lema 3.38 tenemos:

$$
e(\sigma)=\varphi_{\sigma}\left(1_{R}\right) \preceq \sigma \preceq e(\sigma),
$$

de donde $\sigma=e(\sigma)$. Así que por el inciso b) del Teorema 1.82 concluimos que $\sigma$ es idempotente.

Observación 3.40. Si $\sigma$ es un prerradical idempotente, entonces por la Proposición 3.36 y el Lema 3.39:

$$
\sigma=\sigma^{2} \preceq \varphi_{\sigma}(\sigma) \preceq \sigma .
$$

De donde $\varphi_{\sigma}(\sigma)=\sigma=e(\sigma)$.

El siguiente resultado es directo de la Proposición 3.14.

Proposición 3.41. Sean $\sigma, \tau \in R$-pr. Entonces $\varphi_{\sigma}(\tau)=\bigwedge\{\eta \in R$-pr $\mid \sigma \tau \preceq$ $\eta \sigma\}$. 
Demostración. El resultado se tiene de la Proposición 3.14, considerando la transforamción natural $i_{\sigma, \sigma}$.

La siguiente observación muestra que la descripción anterior de $\varphi_{\sigma}(\tau)$ es una buena descripción, pues evaluada en el prerradical $1_{R}$ obtenemos precisamente al igualador de $\sigma$. Ver parte 1 de la Definición 1.81.

\section{Observación 3.42.}

$$
\varphi_{\sigma}\left(1_{R}\right)=\bigwedge\{\eta \in R-p r \mid \sigma \preceq \eta \sigma \preceq \sigma\}=\bigwedge\{\eta \in R-p r \mid \eta \sigma=\sigma\}=e(\sigma) .
$$

La siguiente observación es un resultado un tanto más general que el de la Proposición 3.36.

Observación 3.43. Para $\sigma, \tau \in R$-pr, por la primera parte de la demostración de la Proposición 3.14, se tiene que $\sigma \tau \preceq \varphi_{\sigma}(\tau) \sigma$. De ahi y de las propiedades del producto de prerradicales se tiene que:

$$
\sigma \tau \preceq \varphi_{\sigma}(\tau) \sigma \preceq \varphi_{\sigma}(\tau) .
$$

\subsubsection{Obtención del Coigualador}

Proposición 3.44. Sean $\sigma, \tau \in R$-pr. Entonces $\psi_{\sigma^{*}}(\tau) \preceq(\tau: \sigma)$.

Demostración. Sean $\sigma, \tau \in R$-pr. Por la Proposición 3.13, $\psi_{p_{\sigma, \sigma}}(\tau) \preceq(\tau: \sigma)$. Entonces

$$
\psi_{\sigma^{*}}(\tau)=\psi_{1_{\sigma^{*}}}(\tau)=\varphi_{p_{\sigma, \sigma}}(\tau) \preceq(\tau: \sigma) .
$$

Observación 3.45. Nótese que si $(\tau: \sigma)=(\sigma: \tau)$, entonces $\psi_{\sigma^{*}}(\tau) \succeq \tau$. Puesto que $\psi_{\sigma^{*}}(\tau)=\bigwedge_{M \in R \text {-Mod }} \omega_{\sigma^{*}\left(p_{\tau M}\right)}$ y para $K \in R$-Mod, tenemos

$$
\begin{aligned}
\omega_{\sigma^{*}\left(p_{\tau M}\right)}(K) & =\bigcap\left\{\overleftarrow{f}\left(\operatorname{ker} \sigma^{*}\left(p_{\tau M}\right)\right) \mid f: K \rightarrow M / \sigma(M)\right\} \\
& =\bigcap\{\overleftarrow{f}((\tau: \sigma)(M) / \sigma(M))=f((\sigma: \tau)(M) / \sigma(M)) \mid f: K \rightarrow M / \sigma(M)\} \\
& =\bigcap\{\overleftarrow{f}(\tau(M / \sigma(M))) \mid f: K \rightarrow M / \sigma(M)\}
\end{aligned}
$$

donde $f(\tau(K)) \leq \tau(M / \sigma(M))$, por ser $\tau$ un R-prerradical. De ahí, para toda $f: K \rightarrow M / \sigma(M)$ se tiene que $\overleftarrow{f}(\tau(M / \sigma(M))) \geq \tau(K)$

Lema 3.46. Sea $\sigma \in R$-pr. Entonces $c(\sigma)=\psi_{\sigma^{*}}\left(0_{R}\right)$.

Demostración. Es inmediato de la parte 4 del Corolario 3.26 y del inciso g) del Teorema 1.82.

Lema 3.47. Sea $\sigma \in R$-pr. Entonces $\sigma$ es radical si y sólo si $\psi_{\sigma^{*}}(\tau) \succeq \sigma$ para toda $\tau \in R$-pr. 
Demostración. Sea $\tau \in R$-pr. Entonces $\psi_{\sigma^{*}}(\tau)=\bigwedge_{M \in R \text {-Mod }} \omega_{\sigma^{*}\left(p_{\tau M}\right)}$. Supongamos que $\sigma$ es radical. Sea $K \in R$-Mod, así

$$
\begin{aligned}
\omega_{\sigma^{*}\left(p_{\tau M}\right)}(K) & =\bigcap\left\{\overleftarrow{f}\left(\operatorname{ker}\left(\sigma^{*}\left(p_{\tau M}\right)\right) \mid f: K \rightarrow M / \sigma(M)\right\}\right. \\
& =\bigcap\{\overleftarrow{f}((\tau: \sigma)(M) / \sigma(M)) \mid f: K \rightarrow M / \sigma(M)
\end{aligned}
$$

Para cada $f: K \rightarrow M / \sigma(M)$ por ser $\sigma$ un radical tenemos que $f(\sigma(K)) \leq$ $\sigma(M / \sigma(M))=0$, así que

$$
\overleftarrow{f}((\tau: \sigma)(M) / \sigma(M)) \geq \overleftarrow{f}(0) \geq \sigma(K)
$$

De donde, $\psi_{\sigma^{*}}(\tau) \succeq \sigma$.

Por otro lado, supongamos que $\psi_{\sigma^{*}}(\tau) \succeq \sigma$ para toda $\tau \in R$-pr. Así que considerando $\tau=0_{R}$, por el inciso e) del Teorema 1.82 y el Lema 3.46 tenemos:

$$
c(\sigma)=\psi_{\sigma^{*}}\left(0_{R}\right) \succeq \sigma \succeq c(\sigma),
$$

de donde $\sigma=c(\sigma)$. Luego por el inciso f) del Teorema 1.82 concluimos que $\sigma$ es radical.

Observación 3.48. Si $\sigma$ es radical, entonces por la Proposición 3.44 y el Lema 3.47

$$
\sigma=(\sigma: \sigma) \succeq \psi_{\sigma^{*}}(\sigma) \succeq \sigma .
$$

De donde $\psi_{\sigma^{*}}(\sigma)=\sigma=c(\sigma)$.

El siguiente resultado es consecuencia de la Proposición 3.16.

Proposición 3.49. Sea $\tau \in R$-pr. Entonces $\psi_{\sigma^{*}}(\tau)=\bigvee\{\eta \in R-p r \mid(\sigma: \eta) \preceq$ $(\tau: \sigma)\}$.

Demostración. El resultado se tiene de la Proposición 3.16, considerando la transforamción natural $p_{\sigma, \sigma}$.

La siguiente observación muestra que la descripción anterior de $\psi_{\sigma^{*}}(\tau)$ es una buena descripción, pues evaluada en el prerradical $0_{R}$ obtenemos precisamente el coigualador de $\sigma$. Ver parte 3 de la Definición 1.81 .

\section{Observación 3.50.}

$$
\begin{aligned}
\psi_{\sigma^{*}}\left(0_{R}\right) & =\bigvee\left\{\eta \in R-p r \mid(\sigma: \eta) \preceq\left(0_{R}: \sigma\right)\right\} \\
& =\bigvee\left\{\eta \in R-p r \mid \sigma \preceq(\sigma: \eta) \preceq\left(0_{R}: \sigma\right)=\sigma\right\} \\
& =\bigvee\{\eta \in R-p r \mid(\sigma: \eta)=\sigma\}=c(\sigma) .
\end{aligned}
$$

La siguiente observación es un resultado un poco más general que la Proposición 3.44 . 
Observación 3.51. Para $\sigma, \tau \in R$-pr, por la segunda parte de la demostración de la Proposición 3.16 tenemos que $\left(\sigma: \psi_{\sigma^{*}}(\tau)\right) \preceq(\tau: \sigma)$. De ahí y de las propiedades del coproducto de prerradicales se tiene que:

$$
\psi_{\sigma^{*}}(\tau) \preceq\left(\sigma: \psi_{\sigma^{*}}(\tau)\right) \preceq(\tau: \sigma) .
$$

\subsubsection{Obtención del Anulador}

Lema 3.52. Sea $\sigma \in R$-pr. Entonces $a(\sigma)=\psi_{\sigma}\left(0_{R}\right)$.

Demostración. Es inmediato de la parte 4 del Corolario 3.26 y del inciso d) del Teorema 1.82 .

Proposición 3.53. Sean $\sigma, \tau \in R$-pr. Entonces $\psi_{\sigma}(\tau)=\bigvee\{\eta \in R$-pr $\mid \eta \sigma \preceq$ $\sigma \wedge \tau\}$.

Demostración. Veamos primero que $\psi_{\sigma}(\tau)$ es tal que $\psi_{\sigma}(\tau) \sigma \preceq \sigma \wedge \tau$. Sea $K \in R$-Mod, usando 2 del Teorema 1.63 se sigue:

$$
\begin{aligned}
\left(\psi_{\sigma}(\tau) \sigma\right)(K) & =\left(\bigwedge_{M \in R \text {-Mod }} \omega_{\sigma\left(p_{\tau M}\right)} \sigma\right)(K) \\
& =\bigwedge_{M \in R \text {-Mod }}\left(\omega_{\sigma\left(p_{\tau M}\right)} \sigma\right)(K) \leq \omega_{\sigma\left(p_{\tau K}\right)}(\sigma(K)) .
\end{aligned}
$$

Pero

$$
\begin{aligned}
\omega_{\sigma\left(p_{\tau K}\right)}(\sigma(K)) & =\bigcap\left\{\overleftarrow{f}\left(\operatorname{ker}\left(\sigma\left(p_{\tau K}\right)\right)\right) \mid f: \sigma(K) \rightarrow \sigma(K)\right\} \\
& \leq \overleftarrow{f}\left(\operatorname{ker}\left(\sigma\left(p_{\tau K}\right)\right)\right) \text { para toda } g: \sigma(K) \rightarrow \sigma(K)
\end{aligned}
$$

De lo anterior se sigue en particular que

$$
\begin{aligned}
\omega_{\sigma\left(p_{\tau K}\right)}(\sigma(K)) & \leq \overleftarrow{1_{\sigma(K)}}\left(\operatorname{ker}\left(\sigma\left(p_{\tau K}\right)\right)\right) \\
& =\operatorname{ker}\left(\sigma\left(p_{\tau K}\right)\right)=\sigma(K) \cap \tau(K)
\end{aligned}
$$

donde $1_{\sigma(K)}: \sigma(K) \rightarrow \sigma(K)$ es el morfismo identidad. De lo anterior $\left(\psi_{\sigma}(\tau) \sigma\right)(K) \leq \sigma(K) \cap \tau(K)$. Por lo tanto $\psi_{\sigma}(\tau) \preceq \bigvee\{\eta \in R$-pr $\mid \eta \sigma \preceq \sigma \wedge \tau\}$.

Por otro lado veamos que $\eta \preceq \bigwedge_{M \in R \text {-Mod }} \omega_{\sigma\left(p_{\tau M}\right)}$ para toda $\eta \in R$-pr tal que $\eta \sigma \preceq \sigma \wedge \tau$. Sea $\eta \in R$-pr con la propiedad anterior y $M, K \in R$-Mod. Se tiene que para toda $f: K \rightarrow \sigma(M), f(\eta(K)) \leq \eta(\sigma(M))$ y:

$$
\begin{aligned}
\overleftarrow{f}\left(\operatorname{ker}\left(\sigma\left(p_{\tau M}\right)\right)\right) & =\overleftarrow{f}(\sigma(M) \cap \tau(M)) \\
& \geq \overleftarrow{f}(\eta(\sigma(M))) \geq \eta(K)
\end{aligned}
$$

De lo anterior $\eta \preceq \omega_{\sigma\left(p_{\tau M}\right)}$ para toda $M \in R$-Mod, así que $\eta \preceq \psi_{\sigma}(\tau)$ para toda $\eta$ tal que $\eta \sigma \preceq \sigma \wedge \tau$. De donde concluimos que $\bigvee\{\eta \in R$-pr $\mid \eta \sigma \preceq \sigma \wedge \tau\} \preceq \psi_{\sigma}(\tau)$. Por lo tanto se sigue la igualdad. 


\section{Observación 3.54.}

$$
\begin{aligned}
\psi_{\sigma}\left(0_{R}\right) & =\bigvee\left\{\eta \in R-p r \mid \eta \sigma \preceq \sigma \wedge 0_{R}\right\}=\bigvee\left\{\eta \in R-p r \mid \eta \sigma \preceq 0_{R}\right\} \\
& =\bigvee\left\{\eta \in R-p r \mid \eta \sigma=0_{R}\right\}=a(\sigma) .
\end{aligned}
$$

\subsubsection{Obtención del Totalizador}

Para finalizar este capítulo, mostramos una manera alterna de cómo obtener el totalizador como imagen del prerradical identidad bajo un morfismo $\varphi$ asociado a un funtor, que en este caso, es un funtor inclusión como veremos en seguida.

Sea $\sigma \in R$-pr. Se tiene que $\sigma(R)$ es un ideal bilateral de $R$, ver parte final de la Subsección 1.4.1. Consideremos el funtor inclusión (funtor que olvida) de la categoría $R / \sigma(R)$-Mod a la categoría $R$-Mod, $u_{\sigma}: R / \sigma(R)$-Mod $\rightarrow R$-Mod, asociado a $\sigma$.

Proposición 3.55. Sea $\sigma \in R$-pr. Entonces $t(\sigma)=\varphi_{u_{\sigma}}\left(1_{R / \sigma(R)}\right)$.

Demostración. Se sigue del inciso h) del Teorema 1.82 y de 1 del Corolario 3.26 .

Notemos que en el caso de los operadores, igualador, coigualador y anulador, los morfismos entre retículas de prerradicales que evaluamos en ciertos prerradicales para obtenerlos, estan asociados a funtores que en particular son un prerradical $o$ un coprerradical. En el caso del totalizador el morfismo $\varphi$ esta asociado a un funtor diferente a los que se usa en los otros tres casos, por lo tanto no se obtienen resultados similares a ellos, por ejemplo, en el sentido de mostrar otra descripción general del morfismo $\varphi_{u_{\sigma}}$. 



\section{Capítulo 4}

\section{Morfismos entre $R$-pr y $R / I$-pr}

En este capítulo dado un anillo $R$ asociativo e $I$ un ideal de $R$, estudiaremos la relación que puede haber entre las retículas $R$-pr y $R / I$-pr. Para esto aplicaremos la teoría que hemos desarrollado en los capítulos anteriores a ciertos funtores que involucren a las categorías $R$-Mod y $R / I$-Mod, puesto que si tenemos una situación de adjunción entre dichas categorías tenemos una conexión de Galois entre las retículas $R$-pr y $R / I$-pr y con base en esto podemos estudiar algunas características particulares de dichas retículas. Como casos específicos consideraremos las retículas $\mathbb{Z}_{p^{n}}$-pr con $p$ primo, $n>1$ y aprovechando algunas de sus características estudiaremos a la retícula $\mathbb{Z}$-pr.

\subsection{Conexión de Galois entre las retículas $R$-pr y $R / I$-pr}

Como ya hemos mencionado, trabajaremos con funtores que involucren a las categorías $R$-Mod y $R / I$-Mod. Lo siguiente, nos servirá para definir los funtores en los cuales nos enfocaremos.

Sea $M \in R$-Mod. Entonces $M / I M \in R / I$-Mod, ya que $I \leq \operatorname{ann}_{R}(M / I M)$, donde $\operatorname{ann}_{R}(M / I M)=\{r \in R \mid$ para todo $\bar{x} \in M / I M, r \bar{x} \in I M\}$ es el anulador de $M / I M$ en $R$ ( [2], página 35 ).

Ahora bien, si $f: M \rightarrow N$ es un homomorfismo en $R$-Mod, se tienen las siguientes sucesiones exactas cortas y el diagrama conmutativo:

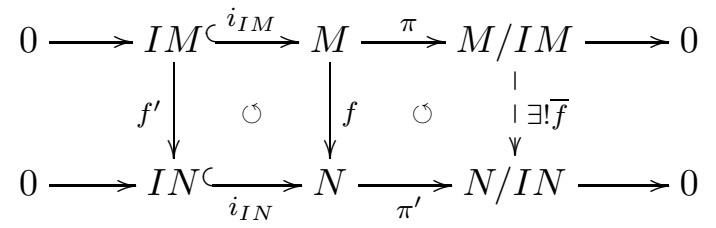

donde $i_{I M}, i_{I N}$ son las inclusiones canónicas y $\pi, \pi^{\prime}$ son las proyecciones canónicas. La existencia del $R / I$-homomorfismo $\bar{f}: M / I M \rightarrow N / I N$ se sigue por el Teorema del Factor puesto que $\pi^{\prime} \circ f \circ i_{I M}=0$.

La siguiente definición introduce los funtores con lo que trabajeremos a lo largo de este capítulo.

Definición 4.1. Sea I un ideal de R. Definimos los siguientes funtores:

1. $F_{I}: R$-Mod $\rightarrow R / I-M o d$, tal que

i) $F_{I}(M)=M / I M$ para cada $M \in R$-Mod.

ii) Si $f: M \rightarrow N$ es un homomorfismo en $R$-Mod, se define $F_{I}(f)=\bar{f}$ :

$M / I M \rightarrow N / I N$, donde $\bar{f}$ se obtiene como en el párrafo anterior. 
2. $G_{I}: R / I-M o d \rightarrow R-M o d$, donde $G_{I}$ es el funtor inclusión de la categoría $R / I$-Mod en la categoría $R$-Mod.

Nos interesa conocer características de las retículas $R$-pr y $R / I$-pr, para ello veremos que $F_{I}$ es un funtor adjunto izquierdo de $G_{I}$ y así poder usar los resultados del Capítulo 2.2.

Proposición 4.2. Sean $F_{I}, G_{I}$, los funtores de la Definición 4.1. Entonces $F_{I}$ : $R$-Mod $\rightarrow R / I$-Mod es un funtor adjunto izquierdo de $G_{I}: R / I$-Mod $\rightarrow R$-Mod.

Demostración. Sean $M \in R$-Mod y $N \in R / I$-Mod. Entonces por el Teorema 1.35 del Capítulo 1, vamos a probar que hay una biyección

$$
\varphi_{M, N}: \operatorname{Hom}_{R / I}\left(F_{I}(M), N\right) \rightarrow \operatorname{Hom}_{R}\left(M, G_{I}(N)\right),
$$

la cual es natural en $M$ y $N$.

Para cada $f \in \operatorname{Hom}_{R / I}(M / I M, N)$, definimos la función:

$$
\varphi_{M, N}: \operatorname{Hom}_{R / I}(M / I M, N) \rightarrow \operatorname{Hom}_{R}(M, N)
$$

como $\varphi_{M, N}(f)=f \circ \pi$, donde $\pi: M \rightarrow M / I M$ es la proyección canónica. Por otro lado, sea $g: M \rightarrow N$ un homomorfismo en $R$-Mod y consideremos el siguiente diagrama conmutativo:

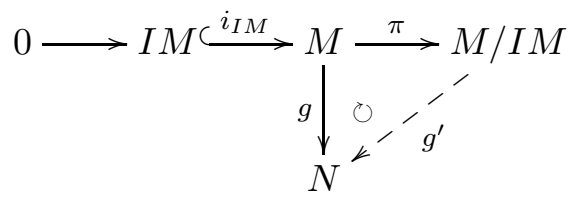

donde $i_{I M}$ es la inclusión canónica y la existencia del $R / I$-homomorfismo $g^{\prime}$ : $M / I M \rightarrow N$ se sigue por el Teorema del Factor, puesto que, completando el diagrama anterior como el diagrama (48) se tiene que $g(I M) \in I N=0$, es decir, $g \circ i_{I M}=0$. Entonces para cada $g \in \operatorname{Hom}_{R}(M, N)$, definimos la función $\psi_{M, N}: \operatorname{Hom}_{R}(M, N) \rightarrow \operatorname{Hom}_{R / I}(M / I M, N)$ como $\psi_{M, N}(g)=g^{\prime}$, donde $g^{\prime}$ es el único homomorfismo tal que $g^{\prime} \circ \pi=g$. No es difícil ver que las funciones $\varphi_{M, N}$ y $\psi_{M, N}$ son inversas entre sí y son naturales en $M$ y $N$.

De ahora en adelante siempre consideramemos un anillo asociativo $R$ e $I$ un ideal de $R$ y siempre usaremos los funtores $F_{I}$ y $G_{I}$ de la Definición 4.1.

Por el Teorema 2.46 de la Sección 2.2 se tiene que la adjunción $\left\langle F_{I}, G_{I}\right\rangle$, induce una conexión de Galois $\left\langle R\right.$-pr, $\varphi_{I}, \psi_{I}, R / I$-pr $\rangle$, donde $\varphi_{I}: R$-pr $\rightarrow R / I$-pr y $\psi_{I}: R / I$-pr $\rightarrow R$-pr. Así que para mencionar características específicas de dicha conexión de Galois, primero veremos las descripciones de $\varphi_{I}$ y $\psi_{I}$ en este caso. En adelante, para referirnos a tal conexión de Galois, usaremos la siguiente notación $\left\langle\varphi_{I}, \psi_{I}\right\rangle$, ver la Observación 1.21.

Proposición 4.3. Sea I un ideal de $R$ y sea $\left\langle\varphi_{I}, \psi_{I}\right\rangle$ la conexión de Galois inducida por la adjunción $\left\langle F_{I}, G_{I}\right\rangle$. Entonces

1. Para cada $\tau \in R$-pr, $\varphi_{I}(\tau)=\bar{\tau}$ es la restricción de $\tau$ en los $R / I$-módulos. 
2. Para cada $\sigma \in R / I$-pr, $\psi_{I}(\sigma)=\tau$ es la mayor extensión de $\sigma$ en los $R$-módulos. Más aún, si $\tau$ es una extensión de $\sigma$ en $R$-Mod, entonces $\psi_{I}(\sigma)=\left(\alpha_{I}^{R}: \tau\right)$.

Demostración. Sea $\tau \in R$-pr y $N \in R / I$-Mod. Por la demostración del Teorema 2.46 de la Sección 2.2, el diagrama para describir $\varphi_{I}: R$-pr $\rightarrow R / I$-pr es el siguiente:

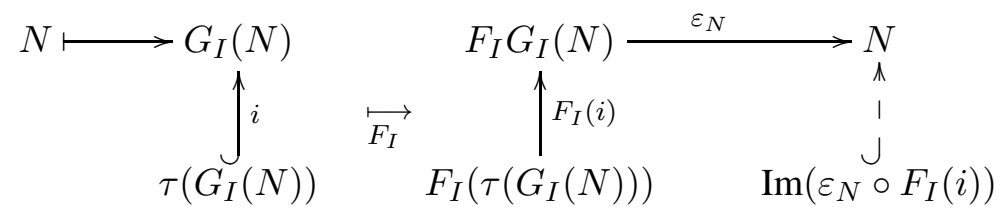

donde en este caso, $i: \tau N \hookrightarrow N$ es la inclusión canónica, $F_{I}(i): \tau(N) / I \tau(N) \rightarrow$ $N / I N$ es la misma inclusión, es decir, $F_{I}(i): \tau N \hookrightarrow N$, puesto que $I \tau(N)=$ $I N=0 \mathrm{y} \varepsilon_{N}=N \rightarrow N$ es el homomorfismo identidad. Entonces, por definición de $\varphi_{I}, \varphi_{I}(\tau)(N):=\operatorname{Im}\left(\varepsilon_{N} \circ F_{I}(i)\right)=\operatorname{Im}\left(F_{I}(i)\right)=\tau(N)$. Concluimos entonces que $\varphi_{I}: R$-pr $\rightarrow R / I$-pr se define para cada $\tau \in R$-pr como:

$$
\varphi(\tau)=\bar{\tau},
$$

donde $\bar{\tau} \in R / I$-pr tal que $\bar{\tau}(N)=\tau(N)$ para $N \in R / I$-Mod. Es decir, $\bar{\tau}$ es la restricción de $\tau$ en los $R / I$-módulos.

Dualmente, sea $\sigma \in R / I$-pr y $M \in R$-Mod. De nuevo por el Teorema 2.46, el diagrama para describir $\psi_{I}: R / I$-pr $\rightarrow R$-pr es el que sigue:
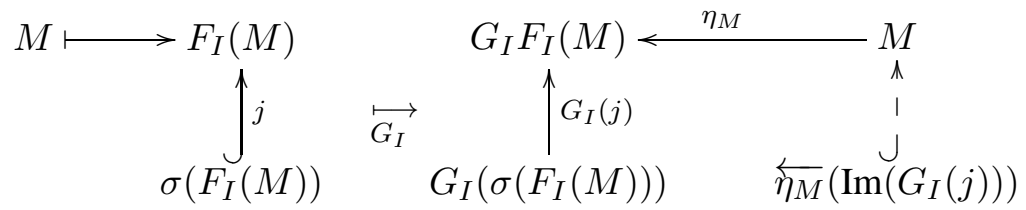

donde en este caso $j: \sigma(M / I M) \hookrightarrow M / I M$ y $G_{I}(j): \sigma(M / I M) \hookrightarrow M / I M$ es la misma inclusión y $\eta_{M}: M \mapsto M / I M$. Entonces, por definición de $\psi_{I}$, $\psi_{I}(\sigma)(M):=\overleftarrow{\eta_{M}}\left(\operatorname{Im}\left(G_{I}(j)\right)\right)=\overleftarrow{\eta_{M}}(\sigma(M / I M))$. También $I M=\operatorname{ker} \eta_{M} \leq$ $\psi_{I}(\sigma)(M)$ y $\psi_{I}(\sigma)(M) / I M=\sigma(M / I M)$. Notemos que si $M \in R / I$-Mod, entonces $\psi_{I}(\sigma)(M)=\sigma(M)$, es decir, $\psi_{I}: R / I$-pr $\rightarrow R$-pr se define para cada $\sigma \in R / I$-pr como:

$$
\psi_{I}(\sigma)=\tau
$$

donde $\tau \in R$-pr es una extensión de $\sigma$ en $R$-Mod. Ahora por la Proposición 1.77, $\alpha_{I}^{R}$ es un $t$-radical tal que $\alpha_{I}^{R}(M)=I M$ para cada $M \in R$-Mod. Si $\tau \in R$-pr es una extensión de $\sigma$ en los $R$-módulos, entonces

$$
\psi_{I}(\sigma)(M) / \alpha_{I}^{R}(M)=\psi_{I}(\sigma)(M) / I M=\sigma(M / I M)=\tau(M / I M) .
$$

De ahí y por la Definición 1.62 del coproducto de prerradicales, se concluye que $\psi_{I}(\sigma)=\left(\alpha_{I}^{R}: \tau\right) \succeq \tau$. 
Como ya hemos visto, las descripciones de $\varphi_{I}$ y $\psi_{I}$ de la conexión de Galois inducida por la adjunción $\left\langle F_{I}, G_{I}\right\rangle$ las obtuvimos usando los diagramas del Teorema 2.46. Por otro lado, del Teorema 2.54, se tiene la siguiente descripción para $\varphi_{I}$ у $\psi_{I}$.

Corolario 4.4. Sea I un ideal de $R$ y sea $\left\langle\varphi_{I}, \psi_{I}\right\rangle$ la conexión de Galois inducida por la adjunción $\left\langle F_{I}, G_{I}\right\rangle$. Entonces

1. Para cada $\tau \in R$-pr, $\varphi_{I}(\tau)=\bigvee_{M \in R-M o d} \alpha_{(\tau(M)+I M) / I M}^{M / I M}$

2. Para cada $\sigma \in R / I-p r, \psi_{I}(\sigma)=\bigwedge_{N \in S \text {-Mod }} \omega_{\sigma N}^{N}$

Demostración. 1. Sea $\tau \in R$-pr. Entonces por el Teorema 2.54:

$$
\varphi_{I}(\tau)=\bigvee_{M \in R \text {-Mod }} \alpha_{F_{I}\left(i_{\tau M}\right)},
$$

donde $i_{\tau M}: \tau(M) \hookrightarrow M$ es la inclusión canónica y $F_{I}\left(i_{\tau M}\right): \tau(M) / I \tau(M) \rightarrow$ $M / I M$. Por lo que

$$
\begin{aligned}
\varphi_{I}(\tau) & =\bigvee_{M \in R-\operatorname{Mod}} \alpha_{\operatorname{Im} F_{I}\left(i_{\tau M}\right)}^{F_{I}(M)} \\
& =\bigvee_{M \in R-\operatorname{Mod}} \alpha_{(\tau M+I M) / I M}^{M / I M}
\end{aligned}
$$

2. Sea $\sigma \in R / I$-pr. Entonces por el Teorema 2.54:

$$
\psi_{I}(\sigma)=\bigwedge_{N \in R / I-\operatorname{Mod}} \omega_{G_{I}\left(p_{\sigma N}\right)}
$$

donde $p_{\sigma N}: N \rightarrow N / \sigma(N)$ es la proyección canónica y $G_{I}\left(p_{\sigma N}\right): N \rightarrow$ $N / \sigma(N)$ es la misma proyección. Entonces

$$
\begin{aligned}
\psi_{I}(\sigma) & =\bigwedge_{N \in R / I-\operatorname{Mod}} \omega_{\operatorname{ker} G_{I}\left(p_{\sigma N}\right)}^{G_{I}(N)} \\
& =\bigwedge_{N \in R / I-\operatorname{Mod}} \omega_{\sigma(N)}^{N}
\end{aligned}
$$

Recordemos las siguientes propiedades que son consecuencia inmediata de la conexión de Galois $\left\langle\varphi_{I}, \psi_{I}\right\rangle$, inducida por la adjunción $\left\langle F_{I}, G_{I}\right\rangle$.

Se tiene que $\psi_{I} \circ \varphi_{I}$ es un operador cerradura en $R$-pr y $\varphi_{I} \circ \psi_{I}$ es un operador interior en $R / I$-pr. También por el Lema 1.19

$$
\psi_{I}\left(1_{R / I}\right)=1_{R}
$$

$\mathrm{y}$

$$
\varphi_{I}\left(0_{R}\right)=0_{R / I} .
$$


Es decir, $1_{R}$ es un elemento cerrado de $R$-pr y $0_{R / I}$ es un elemento abierto de $R / I$-pr, respectivamente.

En este caso, por 1 de la Proposición 4.3, el prerradical idempotente $\sigma_{\varepsilon}$ definido en el Corolario 2.58 es:

$$
\sigma_{\varepsilon}=\varphi_{I}\left(1_{R}\right)=1_{R / I} .
$$

y por 3 del Corolario $2.58,1_{R / I}=\varphi_{I}\left(1_{R}\right)$ es el mayor abierto en $R / I$-pr.

De manera similar, por 2 de la Proposición 4.3, el radical $\tau_{\eta}=\psi_{I}\left(0_{R / I}\right)$ definido en el Corolario 2.58 es la mayor extensión del prerradical $0_{R / I}$ en los $R$-módulos. Así que para cada $M \in R$-Mod por la prueba de la Proposición 4.3 tenemos:

$$
\psi_{I}\left(0_{R / I}\right)(M) / I M=0_{R / I}(M / I M)=0_{R / I-\operatorname{Mod}},
$$

de donde $\psi_{I}\left(0_{R / I}\right)(M)=I M=\alpha_{I}^{R}(M)$, es decir, en ese caso $\tau_{\eta}=\alpha_{I}^{R}$. De ahí y del Corolario 4.4, observamos lo siguiente:

$$
\alpha_{I}^{R}=\psi_{I}\left(0_{R / I}\right)=\bigwedge_{N \in R / I-\operatorname{Mod}} \omega_{0}^{G(N)}=\bigwedge_{N \in R / I \text {-Mod }} \omega_{0}^{N} .
$$

Obteniendo de esta manera una descripción para el $t$-radical $\alpha_{I}^{R}$ y de 6 del Corolario 2.58, sabemos que $\alpha_{I}^{R}=\psi_{I}\left(0_{R / I}\right)$ es el menor cerrado en $R$-pr.

Las siguientes proposiciones describen a los operadores cerradura e interior de la conexión de Galois $\left\langle\varphi_{I}, \psi_{I}\right\rangle$.

Proposición 4.5. $\varphi_{I} \circ \psi_{I}=I d_{R / I-p r}$. Por lo tanto $\psi_{I}$ es inyectiva y $\varphi_{I}$ es sobre.

Demostración. Sea $\sigma \in R / I$-pr y probemos que $\left(\varphi_{I} \circ \psi_{I}\right)(\sigma)=\sigma$. Entonces, para cada $N \in R / I$-Mod por la Proposición 4.3, se tiene que $\varphi_{I}\left(\psi_{I}(\sigma)\right)(N)=$ $\psi_{I}(\sigma)(N)$. Pero $\psi_{I}(\sigma) \in R$-pr, por la prueba de la Proposición 4.3, es tal que $\psi_{I}(\sigma)(N) / I N=\sigma(N / I N)$ y puesto que $N \in R / I$-Mod obtenemos que $\psi_{I}(\sigma)(N)=\sigma(N)$. Por lo tanto $\varphi_{I} \circ \psi_{I}=\operatorname{Id}_{R / I-\mathrm{pr}}$, de donde se concluye que $\psi_{I}$ es inyectiva y $\varphi_{I}$ es sobre.

Ya que $\psi_{I}$ es inyectiva, notemos que cada $\sigma \in R / I$-pr es un elemento abierto respecto al operador interior $\varphi_{I} \circ \psi_{I}$. También se sigue que la imagen de $\psi_{I}$ es isomorfa como clase ordenada a $R / I$-pr $=\operatorname{Im} \varphi_{I}$.

Proposición 4.6. Sea $\tau \in R$-pr. Entonces $\left(\psi_{I} \circ \varphi_{I}\right)(\tau)=\left(\alpha_{I}^{R}: \tau\right)$.

Demostración. Sea $\tau \in R$-pr. Entonces $\left(\psi_{I} \circ \varphi_{I}\right)(\tau)=\psi_{I}\left(\varphi_{I}(\tau)\right)=\psi_{I}(\sigma)$, donde $\varphi_{I}(\tau)=\sigma$ y por la Proposición 4.3, $\sigma$ es la restricción de $\tau$ a los $R / I$-módulos. Es decir, $\tau$ es una extensión de $\sigma$. De nuevo por la Proposición 4.3, concluimos que

$$
\psi_{I}\left(\varphi_{I}(\tau)\right)=\psi_{I}(\sigma)=\left(\alpha_{I}^{R}: \tau\right)
$$


De la Proposición 4.6 y puesto que $\psi_{I} \circ \varphi_{I}$ es un operador cerradura en $R$-pr, se deduce que $\left(\alpha_{I}^{R}:{ }_{-}\right): R$-pr $\rightarrow R$-pr es un operador cerradura. El siguiente resultado es una generalización de lo dicho anteriormente.

Proposición 4.7. Sea $\tau \in R$-rad. Entonces $\left(\tau:{ }_{-}\right): R$-pr $\rightarrow R$-pr es un operador cerradura. También (_ $: \tau): R-p r \rightarrow R$-pr es un operador cerradura.

Demostración. Sea $\tau \in R$-rad. Entonces por la Definición 1.11 del Capítulo 1, probaremos que $\left(\tau:{ }_{-}\right)$es un morfismo de clapos inflatoria y un operador idempotente. Por las propiedades del coproducto de prerradicales es claro que $\left(\tau:{ }_{-}\right)$es un morfismo de clapos inflatoria. Veamos ahora que es un operador idempotente. Sea $\sigma \in R$-pr, puesto que $\tau \in R$-rad y el coproducto es asociativo se tiene que

$$
(\tau:(\tau: \sigma))=((\tau: \tau): \sigma)=(\tau: \sigma) .
$$

Por lo tanto concluimos que $\left(\tau:{ }_{-}\right)$es un operador cerradura. Análogamente se prueba que $(-: \tau)$ es un operador cerradura.

Dualmente tenemos la siguiente proposición.

Proposición 4.8. Sea $\tau \in R$-idem. Entonces $(\tau$. _ $): R$-pr $\rightarrow R$-pr es un operador interior. También $\left(\__{-} \tau\right): R$-pr $\rightarrow R$-pr es un operador interior.

Demostración. La prueba es totalmente dual a la demostración de la Proposición 4.7.

El morfismo $\varphi_{I}$ preserva el producto y el coproducto de prerradicales como veremos enseguida.

Proposición 4.9. Sea $\left\langle\varphi_{I}, \psi_{I}\right\rangle$, la conexión de Galois inducida por la adjunción $\left\langle F_{I}, G_{I}\right\rangle$. Entonces $\varphi_{I}$ preserva productos y coproductos. Es decir, para cada $\tau_{1}, \tau_{2} \in R-p r$

1. $\varphi_{I}\left(\tau_{1} \tau_{2}\right)=\varphi_{I}\left(\tau_{1}\right) \varphi_{I}\left(\tau_{2}\right)$.

2. $\varphi_{I}\left(\tau_{1}: \tau_{2}\right)=\left(\varphi_{I}\left(\tau_{1}\right): \varphi_{I}\left(\tau_{2}\right)\right)$

Demostración. Sean $\tau_{1}, \tau_{2} \in R$-pr y $N \in R / I$-Mod. Entonces por la Proposición 4.3 y puesto que $\varphi_{I}\left(\tau_{2}\right)(N) \in R / I$-Mod, se tiene lo siguiente:

$$
\begin{aligned}
\varphi_{I}\left(\tau_{1} \tau_{2}\right)(N) & =\left(\tau_{1} \tau_{2}\right)(N)=\tau_{1}\left(\tau_{2}(N)\right)=\tau_{1}\left(\varphi_{I}\left(\tau_{2}\right)(N)\right)=\varphi_{I}\left(\tau_{1}\right)\left(\varphi_{I}\left(\tau_{2}\right)(N)\right) \\
& =\left(\varphi_{I}\left(\tau_{1}\right) \varphi_{I}\left(\tau_{2}\right)\right)(N) .
\end{aligned}
$$

Por lo tanto $\varphi_{I}\left(\tau_{1} \tau_{2}\right)=\varphi_{I}\left(\tau_{1}\right) \varphi_{I}\left(\tau_{2}\right)$.

Probemos ahora que $\varphi_{I}\left(\tau_{1}: \tau_{2}\right)=\left(\varphi_{I}\left(\tau_{1}\right): \varphi_{I}\left(\tau_{2}\right)\right)$. Por la Proposición 4.3 y la Definición 1.62 del coproducto de prerradicales, se sigue que:

$$
\begin{aligned}
\varphi_{I}\left(\tau_{1}: \tau_{2}\right)(N) / \varphi_{I}\left(\tau_{1}\right)(N) & =\left(\tau_{1}: \tau_{2}\right)(N) / \tau_{1}(N)=\tau_{2}\left(N / \tau_{1}(N)\right) \\
& =\varphi_{I}\left(\tau_{2}\right)\left(N / \tau_{1}(N)\right)=\varphi_{I}\left(\tau_{2}\right)\left[N / \varphi_{I}\left(\tau_{1}\right)(N)\right] .
\end{aligned}
$$

Por lo tanto $\varphi_{I}\left(\tau_{1}: \tau_{2}\right)=\left(\varphi_{I}\left(\tau_{1}\right): \varphi_{I}\left(\tau_{2}\right)\right)$. Concluimos entonces que $\varphi_{I}$ preserva productos y coproductos. 
Observación 4.10. Recordemos que de la Proposición 2.56 parte 2, para la conexión de Galois entre R-pr y S-pr inducida por una situación de adjunción entre las categorías de módulos respectivas, la asignación $\varphi: R-p r \rightarrow S$-pr preserva prerradicales idempotentes. Como consecuencia de dicho resultado, la asignación $\varphi_{I}$ de la conexión de Galois $\left\langle\varphi_{I}, \psi_{I}\right\rangle$, preserva prerradicales idempotentes, es decir, si $\tau \in R$-idem, entonces $\varphi_{I}(\tau) \in R / I$-idem.

Notemos que tal resultado, también se puede obtener de la Proposición 4.9, parte 1 .

Corolario 4.11. Sea $\left\langle\varphi_{I}, \psi_{I}\right\rangle$, la conexión de Galois inducida por la adjunción $\left\langle F_{I}, G_{I}\right\rangle$. Entonces $\varphi_{I}$ preserva radicales. Es decir, si $\tau$ es un radical, entonces $\varphi_{I}(\tau)$ es un radical.

Demostración. La prueba es inmediata de la Proposición 4.9, parte 2.

Los siguientes corolarios son consecuencia de la Proposición 4.9.

Corolario 4.12. Si en $\overleftarrow{\varphi_{I}}(\sigma)$ hay prerradicales idempotentes con $\sigma \in R / I$-pr, entonces $\sigma$ es un prerradical idempotente.

Demostración. Sea $\tau \in \overleftarrow{\varphi_{I}}(\sigma)$ un prerradical idempotente. Entonces

$$
\sigma=\varphi_{I}(\tau)=\varphi_{I}\left(\tau^{2}\right)
$$

Así que de la igualdad anterior y por la Proposición 4.9 parte 1 , se tiene que

$$
\sigma=\varphi_{I}(\tau) \varphi_{I}(\tau)=\sigma \sigma=\sigma^{2} .
$$

Por lo tanto $\sigma$ es idempotente.

Corolario 4.13. Sea $\sigma \in R / I$-pr. Entonces, $\sigma$ es radical si y sólo si en $\overleftarrow{\varphi_{I}}(\sigma)$ hay radicales.

Demostración. Sea $\sigma \in R / I$-pr radical. Por la Proposición 4.5, tenemos $\varphi_{I}\left(\psi_{I}(\sigma)\right)=\sigma$. Es decir, $\psi_{I}(\sigma) \in \overleftarrow{\varphi_{I}}(\sigma)$ y por 4 de laProposición 2.56, $\psi_{I}(\sigma)$ es radical.

Por otro lado si en $\overleftarrow{\varphi_{I}}(\sigma)$ hay radicales, análogamente a la prueba del Corolario 4.12 , se concluye que $\sigma$ es radical.

\subsection{Particiones de $R$-pr inducidas por un ideal $I$ de $R$}

En esta sección describiremos las imágenes inversas del morfismo $\varphi_{I}$ como intervalos de $R$-pr, las cuales inducen particiones de la retícula $R$-pr que dependen del ideal $I$ al considerar el anillo $R / I$. Introduciremos otra conexión de Galois y veremos que los extremos de los intervalos inducidos por $\overleftarrow{\varphi_{I}}(\sigma)$ con $\sigma \in R / I$-pr, son el resultado de aplicar ciertos morfismos que son parte de las conexiones de Galois que veremos en este trabajo.

La siguiente proposición describe a las imágenes inversas del morfismo $\varphi_{I}$ como intervalos de $R$-pr. En esta descripción aparece el prerradical $t\left(\alpha_{I}^{R}\right)$, ver Lema 
1.83 parte 1 . Antes de enunciar dicha proposición, veamos la siguiente observación.

\section{Observación 4.14.}

1. Notemos que si $N \in R / I$-Mod, entonces $\varphi_{I}\left(t\left(\alpha_{I}^{R}\right)\right)(N)=t\left(\alpha_{I}^{R}\right)(N)=$ $N$, es decir, $\varphi_{I}\left(t\left(\alpha_{I}^{R}\right)\right)=1_{R / I}$.

2. También por el Lema 1.83 parte $2, \alpha_{I}^{R}=a\left(t\left(\alpha_{I}^{R}\right)\right)$, de ahí y de la Definición 1.81 del anulador de un prerradical $\alpha_{I}^{R} t\left(\alpha_{I}^{R}\right)=a\left(t\left(\alpha_{I}^{R}\right)\right) t\left(\alpha_{I}^{R}\right)=0$.

Proposición 4.15. Sea $\left\langle\varphi_{I}, \psi_{I}\right\rangle$, la conexión de Galois inducida por la adjunción $\left\langle F_{I}, G_{I}\right\rangle$. Sea $\sigma \in R / I$-pr. Entonces $\overleftarrow{\varphi_{I}}(\sigma)=\left[\psi_{I}(\sigma) t\left(\alpha_{I}^{R}\right), \psi_{I}(\sigma)\right]$.

Demostración. Sea $\tau \in R$-pr tal que $\tau \in\left[\psi_{I}(\sigma) t\left(\alpha_{I}^{R}\right), \psi_{I}(\sigma)\right]$. Entonces $\psi_{I}(\sigma) t\left(\alpha_{I}^{R}\right) \preceq \tau \preceq \psi_{I}(\sigma)$. Por la demostración del Teorema 2.46, $\varphi_{I}$ preserva orden, así que $\varphi_{I}\left(\psi_{I}(\sigma) t\left(\alpha_{I}^{R}\right)\right) \preceq \varphi_{I}(\tau) \preceq \varphi_{I}\left(\psi_{I}(\sigma)\right)$, de ahí y de las Proposiciones 4.5 y 4.9 obtenemos que

$$
\sigma \varphi_{I}\left(t\left(\alpha_{I}^{R}\right)\right) \preceq \varphi_{I}(\tau) \preceq \sigma .
$$

Por la Observación 4.14 parte $1, \varphi_{I}\left(t\left(\alpha_{I}^{R}\right)\right)=1_{R / I}$, por lo tanto la desigualdad (53) se reduce a $\sigma \preceq \varphi_{I}(\tau) \preceq \sigma$ y entonces $\varphi_{I}(\tau)=\sigma$.

Por otro lado sea $\tau \in R$-pr, tal que $\varphi_{I}(\tau)=\sigma$. Por la Proposición 4.6, el Teorema 1.63 parte 6 que dice que el coproducto es distributivo por la derecha y la Observación 4.14 parte 2 , se tiene:

$$
\begin{gathered}
\psi_{I}(\sigma) t\left(\alpha_{I}^{R}\right)=\psi_{I}\left(\varphi_{I}(\tau)\right) t\left(\alpha_{I}^{R}\right)=\left(\alpha_{I}^{R}: \tau\right) t\left(\alpha_{I}^{R}\right) \preceq\left(\alpha_{I}^{R} t\left(\alpha_{I}^{R}\right): \tau t\left(\alpha_{I}^{R}\right)\right) \\
=\left(0: \tau t\left(\alpha_{I}^{R}\right)\right)=\tau t\left(\alpha_{I}^{R}\right) .
\end{gathered}
$$

De donde

$$
\psi_{I}(\sigma) t\left(\alpha_{I}^{R}\right) \preceq \tau t\left(\alpha_{I}^{R}\right) \preceq \tau \preceq\left(\alpha_{I}^{R}: \tau\right)=\psi_{I}(\sigma)
$$

Por lo tanto $\overleftarrow{\varphi_{I}}(\sigma)=\left[\psi_{I}(\sigma) t\left(\alpha_{I}^{R}\right), \psi_{I}(\sigma)\right]$

Los intervalos que aparecen en la Proposición 4.15, se pueden describir de otra manera, a saber, en términos de un elemento de dicho intervalo y en donde los extremos son el producto y el coproducto de ciertos prerradicales como veremos en la siguiente proposición.

Proposición 4.16. Sea $\left\langle\varphi_{I}, \psi_{I}\right\rangle$, la conexión de Galois inducida por la adjunción $\left\langle F_{I}, G_{I}\right\rangle$. Si $\tau \in \overleftarrow{\varphi_{I}}(\sigma)$, con $\sigma \in R / I$-pr, entonces $\overleftarrow{\varphi_{I}}(\sigma)=\left[\tau t\left(\alpha_{I}^{R}\right),\left(\alpha_{I}^{R}: \tau\right)\right]$

Demostración. Sea $\tau \in \overleftarrow{\varphi_{I}}(\sigma)$. Entonces por (54) de la demostración de la Proposición 4.15, $\psi_{I}(\sigma) t\left(\alpha_{I}^{R}\right) \preceq \tau t\left(\alpha_{I}^{R}\right)$. Puesto que $\tau \preceq\left(\alpha_{I}^{R}: \tau\right)$ y por la Proposición 4.6, obtenemos $\tau t\left(\alpha_{I}^{R}\right) \preceq\left(\alpha_{I}^{R}: \tau\right) t\left(\alpha_{I}^{R}\right)=\psi_{I}(\sigma) t\left(\alpha_{I}^{R}\right)$. De donde, $\psi_{I}(\sigma) t\left(\alpha_{I}^{R}\right)=\tau \alpha_{I}^{R}$. Luego, por la Proposiciones 4.6 y 4.15

$$
\begin{aligned}
\overleftarrow{\varphi_{I}}(\sigma) & =\left[\psi_{I}(\sigma) t\left(\alpha_{I}^{R}\right), \psi_{I}(\sigma)\right]=\left[\tau t\left(\alpha_{I}^{R}\right), \psi_{I}\left(\varphi_{I}(\tau)\right)\right] \\
& =\left[\tau t\left(\alpha_{I}^{R}\right),\left(\alpha_{I}^{R}: \tau\right)\right] .
\end{aligned}
$$


Los siguientes resultados son consecuencia de la Proposición 4.15.

Corolario 4.17. $\overleftarrow{\varphi_{I}}\left(0_{R / I}\right)=\left[0_{R}, \alpha_{I}^{R}\right]$

Demostración. De la ecuación (52) y la Proposición 4.15 tenemos que

$$
\overleftarrow{\varphi_{I}}\left(0_{R / I}\right)=\left[\psi_{I}\left(0_{R / I}\right) t\left(\alpha_{I}^{R}\right), \psi_{I}\left(0_{R / I}\right)\right]=\left[\alpha_{I}^{R} t\left(\alpha_{I}^{R}\right), \alpha_{I}^{R}\right]
$$

Luego, por la Observación 4.14 parte 2 , se concluye que $\overleftarrow{\varphi_{I}}\left(0_{R / I}\right)=\left[0_{R}, \alpha_{I}^{R}\right]$.

Del Corolario 4.17, notemos que $\varphi_{I}\left(\alpha_{I}^{R}\right)=0_{R / I}$.

Corolario 4.18. $\overleftarrow{\varphi_{I}}\left(1_{R / I}\right)=\left[t\left(\alpha_{I}^{R}\right), 1_{R}\right]$

Demostración. Por la Proposición 4.15 se tiene que

$$
\overleftarrow{\varphi_{I}}\left(1_{R / I}\right)=\left[\psi_{I}\left(1_{R / I}\right) t\left(\alpha_{I}^{R}\right), \psi_{I}\left(1_{R / I}\right)\right]
$$

De la ecuación (49), $\psi_{I}\left(1_{R / I}\right)=1_{R}$, por lo tanto concluimos que $\overleftarrow{\varphi_{I}}\left(1_{R / I}\right)=$ $\left[t\left(\alpha_{I}^{R}\right), 1_{R}\right]$.

Del corolario 4.18, observamos que $\varphi_{I}\left(t\left(\alpha_{I}^{R}\right)\right)=1_{R / I}$.

La siguiente proposición coincide con la descripción de la Proposición 4.16. Es decir, los extremos superiores de los intervalos que resultan de las imágenes inversas bajo $\varphi_{I}$, son elementos cerrados.

Proposición 4.19. Sea $\left\langle\varphi_{I}, \psi_{I}\right\rangle$, la conexión de Galois inducida por la adjunción $\left\langle F_{I}, G_{I}\right\rangle$. Sea $\gamma \in \overleftarrow{\varphi_{I}}(\sigma)=\left[\psi_{I}(\sigma) t\left(\alpha_{I}^{R}\right), \psi_{I}(\sigma)\right]$ con $\sigma \in R / I$-pr. Entonces $\psi_{I}(\sigma)$ es la cerradura de $\gamma$, respecto al operador cerradura $\psi_{I} \circ \varphi_{I}$.

Demostración. Sea $\gamma \in\left[\psi_{I}(\sigma) t\left(\alpha_{I}^{R}\right), \psi_{I}(\sigma)\right]$. Entonces $\varphi_{I}(\gamma)=\sigma$. Puesto que $\psi_{I} \circ \varphi_{I}$ es un operador cerradura obtenemos lo siguiente

$$
\gamma \leq\left(\psi_{I} \circ \varphi_{I}\right)(\gamma)=\psi_{I}\left(\varphi_{I}(\gamma)\right)=\psi_{I}(\sigma) .
$$

Por la Definición 1.12, concluimos que $\psi_{I}(\sigma)$ es la cerradura de $\gamma$.

También, puesto que $t\left(\alpha_{I}^{R}\right)$ es un prerradical idempotente, por la Proposición 4.8 observamos que los extremos inferiores de los intervalos son elementos abiertos respecto al operador interior $\left(\ldots \cdot t\left(\alpha_{I}^{R}\right)\right)$.

Notemos que por la Observación 1.52 y el Lema 1.84, la traza con respecto al $R$-módulo $R / I$ es $\alpha_{R / I}^{R / I}=t\left(\alpha_{I}^{R}\right)$. Por lo que, por la Proposición 4.15 para $\sigma \in R / I$-pr, $\overleftarrow{\varphi_{I}}(\sigma)=\left[\psi_{I}(\sigma) \alpha_{R / I}^{R / I}, \psi_{I}(\sigma)\right]$

Observación 4.20. Por la Proposición 4.3, si $\tau \in R$-pr, entonces $\varphi_{I}(\tau)=\sigma$ es la restricción de $\tau$ a los $R / I$-módulos, o bien, $\tau$ es una extensión de $\sigma$ a los $R$ módulos, así que $\overleftarrow{\varphi_{I}}(\sigma)$ es la clase de todos los prerradicales que son extensión de $\sigma$ a los R-módulos. Por la Proposición 4.16, $\tau \alpha_{R / I}^{R / I}$ y $\left(\alpha_{I}^{R}: \tau\right)$ son la menor y mayor extensión respectivamente de $\sigma$ a los $R$-módulos, lo cuál coincide con la Proposición 4.3, parte 2. 
Los intervalos $\overleftarrow{\varphi_{I}}(\sigma)$, con $\sigma \in R / I$-pr forman una partición de $R$-pr. Esta partición coincide con la partición de $R$-pr dada por una clase de módulos, como veremos en lo que sigue. Recordemos de la Sección 1.4.2 del Capítulo 1, que $[\tau]_{\mathcal{C}}$, denota la clase de equivalencia de $\tau \in R$-pr respecto a una clase $\mathcal{C} \subseteq R$-Mod.

Sea $I$ un ideal de $R$ y consideremos la clase $\mathcal{C}_{I}$ de todos los $R$-módulos que son anulados por $I$, es decir,

$$
\mathcal{C}_{I}=\{M \in R-\operatorname{Mod} \mid I M=0\} .
$$

Notemos que la clase $\mathcal{C}_{I}$ es la de los $R / I$-módulos, vista como subcategoría de $R$-Mod. La clase de equivalencia de $[\tau]_{\mathcal{C}_{I}}$ con $\tau \in R$-pr, es la clase de todos los prerradicales cuya restricción a los $R / I$-módulos es igual a la de $\tau$.

Proposición 4.21. Sea $\sigma \in R / I$-pr tal que $\varphi_{I}(\tau)=\sigma$, para cierta $\tau \in R$-pr. Entonces $[\tau]_{\mathcal{C}_{I}}=\overleftarrow{\varphi_{I}}(\sigma)$

Demostración. Sea $\sigma \in R / I$-pr. Entonces por la Proposición 4.5, existe $\tau \in R$ pr tal que $\varphi_{I}(\tau)=\sigma$. Sea $\tau_{1} \in R$-pr tal que $\tau_{1} \in[\tau]_{\mathcal{C}_{I}}$. Entonces por la Definición 1.85, para todo $M \in \mathcal{C}_{I}, \tau_{1}(M)=\tau(M)$ y por la definición de $\varphi_{I}$ :

$$
\varphi_{I}\left(\tau_{1}\right)(M)=\tau_{1}(M)=\tau(M)=\varphi_{I}(\tau)(M) .
$$

De donde, $\varphi_{I}\left(\tau_{1}\right)(M)=\sigma(M)$. Es decir, $\tau_{1} \in \overleftarrow{\varphi_{I}}(\sigma)$. Por otro lado, si $\tau_{1} \in$ $\overleftarrow{\varphi_{I}}(\sigma)$, entonces $\varphi_{I}\left(\tau_{1}\right)=\sigma=\varphi_{I}(\tau)$. Así que para todo $N \in R / I$-Mod:

$$
\tau_{1}(N)=\varphi_{I}\left(\tau_{1}\right)(N)=\varphi_{I}(\tau)(N)=\tau(N),
$$

de donde concluimos que $\tau_{1} \in[\tau]_{\mathcal{C}_{I}}$. Por lo tanto $[\tau]_{\mathcal{C}_{I}}=\overleftarrow{\varphi_{I}}(\sigma)$

De aquí en adelante, denotaremos a la clase de equivalencia de $\tau \in R$-pr con respecto a la clase $\mathcal{C}_{I} \subseteq R$-Mod, vista en (55), donde $I$ es un ideal fijo de $R$, como:

$$
[\tau]_{I}=[\tau]_{\mathcal{C}_{I}}
$$

Por la Proposición 1.87 del Capítulo 1 y las Proposiciones 4.16, 4.21, tenemos varias maneras para describir a los intervalos que inducen las imágenes inversas de $\varphi_{I}$ o bien las clases de equivalencia de $R$-pr, respecto a la clase $\mathcal{C}_{I}$ como vemos a continuación.

Sea $\tau \in \overleftarrow{\varphi_{I}}(\sigma)$, con $\sigma \in R / I$-pr. Entonces

$$
\begin{aligned}
{\left[\tau t\left(\alpha_{I}^{R}\right),\left(\alpha_{I}^{R}: \tau\right)\right] } & =\overleftarrow{\varphi_{I}}(\sigma)=[\tau]_{I}=\left[\bigvee_{M \in \mathcal{C}_{I}} \alpha_{\tau M}^{M}, \bigwedge_{M \in \mathcal{C}_{I}} \omega_{\tau M}^{M}\right] \\
& =\left[\bigvee_{M \in R / I-\operatorname{Mod}} \alpha_{\sigma M}^{M}, \bigwedge_{M \in R / I-\operatorname{Mod}} \omega_{\sigma M}^{M}\right]
\end{aligned}
$$

En la última igualdad $\sigma \in R / I$-pr, por lo que consideramos a $M$ un $R / I$ módulo, puesto que $M \in \mathcal{C}_{I}$. 
Observando la Proposición 4.15 y lo anterior notamos que $\psi_{I}(\sigma)=\bigwedge_{M \in R / I \text {-Mod }} \omega_{\sigma M}^{M}$, lo cual coincide con la definición de tal morfismo. Del mismo modo

$$
\psi_{I}(\sigma) t\left(\alpha_{I}^{R}\right)=\psi_{I}(\sigma) \alpha_{R / I}^{R / I}=\bigvee_{M \in R / I-\operatorname{Mod}} \alpha_{\sigma M}^{M},
$$

lo cual nos induce a pensar que el extremo inferior del intervalo es el resultado de aplicar un morfismo inducido por un funtor. De hecho, como ya habíamos notado antes por la Proposición $4.8,\left(-\cdot \alpha_{R / I}^{R / I}\right)$ es un operador interior que corresponde a cierta conexión de Galois.

Sea $H_{I}: R$-Mod $\rightarrow R / I$-Mod el funtor definido para cada $M \in R$-Mod como $H_{I}(M)=\alpha_{R / I}^{R / I}(M)=\operatorname{ann}_{I}(M)$ y $G_{I}: R / I$-Mod $\rightarrow R$-Mod, el funtor inclusión de la categoría $R / I$-Mod en la categoría $R$-Mod, visto en la Definición 4.1.

Proposición 4.22. El funtor $G_{I}$ es un funtor adjunto izquierdo de $H_{I}: R$-Mod $\rightarrow$ $R / I$-Mod.

Demostración. Sean $M \in R$-Mod y $N \in R / I$-Mod. Entonces por el Teorema 1.35 del Capítulo 1, vamos a probar que hay una biyección

$$
\varphi_{N, M}: \operatorname{Hom}_{R}\left(G_{I}(N), M\right) \rightarrow \operatorname{Hom}_{R / I}\left(N, H_{I}(M)\right),
$$

la cual es natural en $N$ y $M$.

Para cada $f \in \operatorname{Hom}_{R}(N, M)$, definimos la función $\varphi_{N, M}: \operatorname{Hom}_{R}(N, M) \rightarrow$ $\operatorname{Hom}_{R / I}\left(N, \alpha_{R / I}^{R / I}(M)\right)$ como $\varphi_{N, M}(f)=g$, donde $g: N \rightarrow \alpha_{R / I}^{R / I}(M)$ se define para cada $n \in N$ como $g(n)=f(n)$, la cual esta bien definida puesto que $f(n) \in$ $\alpha_{R / I}^{R / I}=t\left(\alpha_{I}^{R}\right)(M)$.

Ahora para cada $g \in \operatorname{Hom}_{R / I}\left(N, t\left(\alpha_{I}^{R}\right)(M)\right)$, definimos la función $\psi_{N, M}$ : $\operatorname{Hom}_{R / I}\left(N, t\left(\alpha_{I}^{R}\right)(M)\right) \rightarrow \operatorname{Hom}_{R}(N, M)$ como $\psi_{N, M}(g)=i_{t} \circ g$, donde $i_{t}:$ $t\left(\alpha_{I}^{R}\right)(M) \hookrightarrow M$. No es difícil ver que las funciones $\varphi_{N, M}$ y $\psi_{N, M}$ son inversas entre sí y son naturales en $M$ y $N$.

Por el el Teorema 2.46 de la Sección 2.2, se tiene que la adjunción $\left\langle G_{I}, H_{I}\right\rangle$ induce una conexión de Galois $\left\langle R / I\right.$-pr, $\zeta_{I}, \xi_{I}, R$-pr $\rangle$. Para tal conexión de Galois, usaremos la notación $\left\langle\zeta_{I}, \xi_{I}\right\rangle$. Veamos las descripciones de los morfismos $\zeta_{I}$ : $R / I$-pr $\rightarrow R$-pr y $\xi_{I}: R$-pr $\rightarrow R / I$-pr

Proposición 4.23. Sea I un ideal de $R$ y sea $\left\langle\zeta_{I}, \xi_{I}\right\rangle$ la conexión de Galois inducida por la adjunción $\left\langle G_{I}, H_{I}\right\rangle$. Entonces

1. Para cada $\sigma \in R / I-p r, \zeta_{I}(\sigma)$ es la menor extensión de $\sigma$ en los $R$-módulos. Más aún, si $\tau$ es una extensión de $\sigma$ en R-Mod, entonces $\zeta_{I}(\sigma)=$ $\tau \alpha_{R / I}^{R / I}$.

2. Para cada $\tau \in R$-pr, $\xi_{I}(\tau)$ es la restricción de $\tau$ en los $R$-módulos, es decir, $\xi_{I}(\tau)=\varphi_{I}(\tau)$. 
Demostración. Sea $M \in R$-Mod y $\sigma \in R / I$-pr, por la demostración del Teorema 2.46, el diagrama para describir $\zeta_{I}$ es el siguiente:

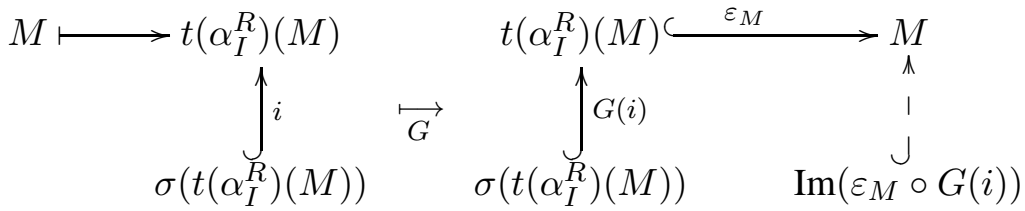

donde $i: \sigma\left(t\left(\alpha_{I}^{R}\right)(M)\right) \hookrightarrow t\left(\alpha_{I}^{R}\right)$ es la inclusión canónica, $G(i)$ es la misma inclusión y $\varepsilon_{M}: t\left(\alpha_{I}^{R}\right) \hookrightarrow M$ es la inclusión canónica. Entonces $\zeta_{I}(\sigma)(M):=\operatorname{Im}\left(\varepsilon_{M} \circ\right.$ $G(i))=\sigma\left(t\left(\alpha_{I}^{R}\right)(M)\right)$. Notemos que si $N \in R / I$-mod, entonces $\zeta_{I}(\sigma)(N)=$ $\sigma\left(t\left(\alpha_{I}^{R}\right)(N)\right)=\sigma(N)$. Por lo tanto

$$
\zeta_{I}(\sigma)=\tau,
$$

donde $\tau \in R$-pr, tal que $\tau(N)=\sigma(N)$ para toda $N \in R / I$-Mod. Es decir, $\tau$ es una extensión de $\sigma$ en los $R$-módulos. Si $\tau \in R$-pr es una extensión de $\sigma$ en los $R$-módulos, entonces para cada $M \in R$-Mod,

$$
\zeta_{I}(\sigma)(M)=\sigma\left(t\left(\alpha_{I}^{R}\right)(M)\right)=\tau\left(t\left(\alpha_{I}^{R}\right)(M)\right)=\left(\tau \alpha_{R / I}^{R / I}\right)(M) \preceq \tau(M),
$$

por lo tanto $\zeta_{I}(\sigma)=\tau \alpha_{R / I}^{R / I} \preceq \tau$.

Por otro lado sea $N \in R / I$-Mod y $\tau \in R$-pr. Usando el Teorema 2.46, el diagrama para describir $\xi_{I}$ es el siguiente:

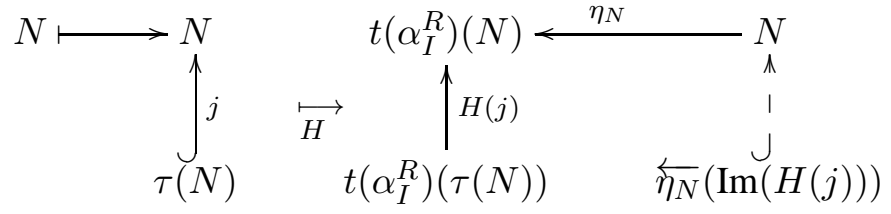

donde $j: \tau(N) \hookrightarrow N$ es la inclusión canónica y puesto que $N \in R / I$-Mod, se tiene que $t\left(\alpha_{I}^{R}\right)(N)=N$ y $t\left(\alpha_{I}^{R}\right)(\tau N)=\tau(N)$. Por lo tanto, $H(j)$ es la misma inclusión $j$ y $\eta_{N}: N \rightarrow N$ es el homomorfismo identidad. Entonces $\psi(\tau)(N):=$ $\overleftarrow{\eta_{N}}(\operatorname{Im}(H(j)))=\overleftarrow{\eta_{N}}(\tau N)=\tau(N)$. Es decir

$$
\xi_{I}(\tau)=\sigma,
$$

donde $\sigma \in R / I$-pr, tal que $\sigma(N)=\tau(N)$ para toda $N \in R / I$-Mod. Es decir, $\sigma$ es una restricción de $\tau$ en los $R / I$-módulos. Notemos que por la Proposición 4.3, se tiene que

$$
\xi_{I}(\tau)=\varphi_{I}(\tau)
$$

para toda $\tau \in R$-pr.

Usando el Teorema 2.54 se tiene otra manera de describir a los morfismos $\zeta_{I}$ y $\xi_{I}$ que son parte de la conexión de Galois inducida por la adjunción $\left\langle G_{I}, H_{I}\right\rangle$.

Corolario 4.24. Sea I un ideal de $R$ y sea $\left\langle\zeta_{I}, \xi_{I}\right\rangle$ la conexión de Galois inducida por la adjunción $\left\langle G_{I}, H_{I}\right\rangle$. Entonces: 
1. Para cada $\sigma \in R / I$-pr, $\zeta_{I}(\sigma)=\bigvee_{N \in R / I-M o d} \alpha_{\sigma N}^{N}$

2. Para cada $\tau \in R-p r, \xi_{I}(\tau)=\bigwedge_{M \in R-M o d} \omega_{a n n_{I}(M) \cap \tau(M)}^{a n n_{I}(M)}$

DEMOSTRACIÓN.

Sea $\sigma \in R / I$-pr. Entonces por el Teorema 2.54, $\zeta_{I}(\sigma)=\bigvee_{N \in R / I \text {-Mod }} \alpha_{G\left(i_{\sigma N}\right)}$, donde $i_{\sigma N}: \sigma(N) \hookrightarrow N$ es la inclusión natural y $G\left(i_{\sigma N}\right): \sigma(N) \hookrightarrow N$ es la misma inclusión, así que

$$
\begin{aligned}
\zeta_{I}(\sigma) & =\bigvee_{N \in R / I-\operatorname{Mod}} \alpha_{\operatorname{Im} G\left(i_{\sigma N}\right)}^{G(N)} \\
& =\bigvee_{N \in R / I-\operatorname{Mod}} \alpha_{\sigma(N)}^{N},
\end{aligned}
$$

Por lo tanto

$$
\zeta_{I}(\sigma)=\tau,
$$

donde $\tau \in R$-pr tal que $\tau(N)=\sigma(N)$, para todo $N \in R / I$-Mod. Es decir, $\tau$ es una extensión de $\sigma$ en los $R$-módulos, lo cual coincide con lo visto en (58).

Por otro lado sea $\tau \in R$-pr. Entonces por el Teorema 2.54, $\xi_{I}(\tau)=\bigwedge_{M \in R \text {-Mod }} \omega_{H\left(p_{\tau M}\right)}$, donde $p_{\tau M}: M \rightarrow M / \tau(M)$ es la proyección natural y $H\left(p_{\tau M}\right): \alpha_{R / I}^{R / I}(M) \rightarrow \alpha_{R / I}^{R / I}(M / \tau(M))$, de ahí:

$$
\begin{aligned}
\xi_{I}(\tau) & =\bigwedge_{M \in R \text {-Mod }} \omega_{\operatorname{ker} H\left(p_{\tau M}\right)}^{H(M)}=\bigwedge_{M \in R \text {-Mod }} \omega_{\alpha_{R / I}^{R / I}(M) \cap \tau(M)}^{\alpha_{R / I}^{R / I}(M)} \\
& =\bigwedge_{M \in R \text {-Mod }} \omega_{\operatorname{ann}_{I}(M) \cap \tau(M)}^{\operatorname{ann}_{I}(M)}
\end{aligned}
$$

Por ser $\left\langle R / I\right.$-pr $, \zeta_{I}, \xi_{I}, R$-pr $\rangle$ una conexión de Galois, recordemos que $\xi_{I} \circ \zeta_{I}$ es un operador cerradura y $\zeta_{I} \circ \xi_{I}$ es un operador interior. Los siguientes corolarios son los duales a las proposiciones 4.5 y 4.6 .

Corolario 4.25. Sea I un ideal de $R$ y $\left\langle\zeta_{I}, \xi_{I}\right\rangle$ la conexión de Galois inducida por la adjunción $\left\langle G_{I}, H_{I}\right\rangle$. Entonces $\xi_{I} \circ \zeta_{I}=I d_{R / I-p r}$. Por lo tanto $\zeta_{I}$ es inyectiva $y$ $\xi_{I}$ es sobre.

Demostración. Sea $\sigma \in R / I$-pr y $N \in R / I$-Mod, probemos que $\left(\xi_{I} \circ \zeta_{I}\right)(\sigma)=$ $\sigma$. Entonces, por la Proposición 4.23 parte 2, $\xi_{I}\left(\zeta_{I}(\sigma)\right)(N)=\zeta_{I}(\sigma)(N)$. De nuevo, usando la Proposición 4.23 parte $1, \zeta_{I}(\sigma)(N)=\sigma(N)$, de donde se concluye el resultado.

Notemos que todos los elementos de $R / I$-pr son cerrados respecto al operador cerradura $\xi_{I} \circ \zeta_{I}$, puesto que $\zeta_{I}$ es inyectiva.

Ahora veamos otra descripción para el operador interior $\zeta_{I} \circ \xi_{I}$. 
Corolario 4.26. Sea I un ideal de $R$ y $\left\langle\zeta_{I}, \xi_{I}\right\rangle$ la conexión de Galois inducida por la adjunción $\left\langle G_{I}, H_{I}\right\rangle$. Entonces, para cada $\tau \in R-p r,\left(\zeta_{I} \circ \xi_{I}\right)(\tau)=\tau t\left(\alpha_{I}^{R}\right)$.

Demostración. Sea $\tau \in R$-pr. Por la Proposición 4.23 parte 2,

$$
\left(\zeta_{I} \circ \xi_{I}\right)(\tau)=\zeta_{I}\left(\xi_{I}(\tau)\right)=\zeta_{I}\left(\varphi_{I}(\tau)\right)=\zeta_{I}(\sigma),
$$

donde $\sigma \in R / I$-pr, tal que $\varphi_{I}(\tau)=\sigma$. Es decir, $\tau$ es una extensión de $\sigma$ en los $R$-módulos y por la Proposición 4.23 parte 1, la igualdad anterior se reduce a $\left(\zeta_{I} \circ \xi_{I}\right)(\tau)=\zeta_{I}(\sigma)=\tau \alpha_{R / I}^{R / I}$, de donde se obtiene el resultado.

Observación 4.27. Sea $\tau \in \overleftarrow{\varphi_{I}}(\sigma)$, con $\sigma \in R / I$-pr. Como ya habíamos mencionado en la Igualdad (57), en efecto los extremos inferiores de $\overleftarrow{\varphi_{I}}(\sigma)$, son el resultado de aplicar un morfismo inducido por un funtor, a saber, $\zeta_{I}$ :

$$
\psi_{I}(\sigma) t\left(\alpha_{I}^{R}\right)=\bigvee_{M \in R / I-M o d} \alpha_{\sigma M}^{M}=\zeta_{I}(\sigma)
$$

También

$$
\begin{aligned}
\overleftarrow{\varphi_{I}}(\sigma) & =\left[\left(\zeta_{I} \circ \xi_{I}\right)(\tau),\left(\psi_{I} \circ \varphi_{I}\right)(\tau)\right] \\
& =\left[\left(\zeta_{I} \circ \varphi_{I}\right)(\tau),\left(\psi_{I} \circ \varphi_{I}\right)(\tau)\right]
\end{aligned}
$$

Finalizaremos este capítulo con un resultado que compara la partición de $R$-pr inducida por las imágenes inversas bajo el morfismo $\varphi_{I}$ que depende del ideal $I$ de $R$ con otra partición de $R$-pr que es inducida por un ideal $J \subseteq I$ de $R$. Para esto, presentamos la siguiente notación.

Notación. Sea $I$ un ideal de $R$. Denotamos a la partición $\mathcal{P}_{I}=\left\{[\tau]_{I} \mid \tau \in\right.$ $R$-pr\}.

Proposición 4.28. Sean $I, J$ ideales de R. Si $J \subseteq I$, entonces $[\tau]_{J} \subseteq[\tau]_{I}$ para toda $\tau \in R$-pr. Es decir, la partición $\mathcal{P}_{J}$ es más fina que la partición $\mathcal{P}_{I}$.

Demostración. Sea $\tau \in \overleftarrow{\varphi_{I}}(\sigma)$, con $\sigma \in R / I$-pr. Por (56), se tiene que $[\tau]_{I}=$ $\overleftarrow{\varphi_{F}}(\sigma)=\left[\tau t\left(\alpha_{I}^{R}\right),\left(\alpha_{I}^{R}: \tau\right)\right]$. Puesto que $J \subseteq I$, tenemos que $\alpha_{J}^{R} \preceq \alpha_{I}^{R}$ y por tanto $\left(\alpha_{J}^{R}: \tau\right) \preceq\left(\alpha_{I}^{R}: \tau\right)$. Para cada $M \in R$-Mod, por el Lema 1.83 y puesto que $J \subseteq I$, obtenemos que $t\left(\alpha_{I}^{R}\right) \preceq t\left(\alpha_{J}^{R}\right)$. Por tanto $\tau t\left(\alpha_{I}^{R}\right) \preceq \tau t\left(\alpha_{J}^{R}\right)$. Concluimos entonces que

$$
\left[\tau t\left(\alpha_{J}^{R}\right),\left(\alpha_{J}^{R}: \tau\right)\right] \subseteq\left[\tau t\left(\alpha_{I}^{R}\right),\left(\alpha_{I}^{R}: \tau\right)\right] .
$$

Es decir, la partición de $R$-pr que se obtiene con el ideal $J$ es más fina que la partición que se obtiene con el ideal $I$. 


\section{Capítulo 5}

\section{Aplicaciones a grupos abelianos}

Como una aplicación a la teoría desarrollada en el capítulo anterior, en la primera parte de esta sección estudiaremos la conexión de Galois entre las retículas $R$-pr y $R / I$-pr, considerando el ideal $I=p^{n} \mathbb{Z}$ del anillo $\mathbb{Z}$ con $p$ primo y $n \geq 1$. En este caso particular interpretaremos algunos resultados generales a la conexión

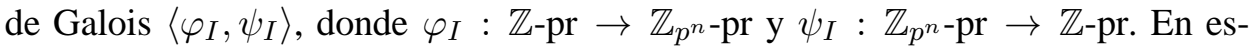
pecial, describiremos la partición de $\mathbb{Z}$-pr inducida por el ideal $I$ de $\mathbb{Z}$, así como los intervalos en $\mathbb{Z}$-pr inducidos por las imágenes inversas bajo $\varphi_{I}$ de prerradicales idempotentes y radicales en $\mathbb{Z}_{p^{n}}$-pr. Este estudio en cierta forma queda conveniente, puesto que en [7] se caracteriza la retícula $\mathbb{Z}_{p^{n}}$-pr y aprovechamos que

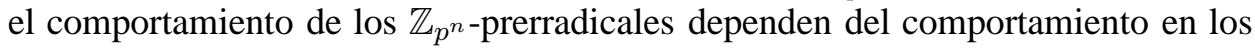
ideales del anillo $\mathbb{Z}_{p^{n}}$. Aprovechando un poco más tal retícula, también veremos la conexión de Galois $\left\langle\varphi_{I}, \psi_{I}\right\rangle$, para el caso en el que $R=\mathbb{Z}_{p^{n}}$ e $I=p^{i} \mathbb{Z}_{p^{n}}$ con $p$ primo, $n \geq 2$ e $i=1, \ldots, n-1$. Por último, consideraremos el anillo $\mathbb{Z}_{(p)}$ que es la localización del anillo $\mathbb{Z}$ en el primo $p$.

\subsection{Particiones de $\mathbb{Z}$-pr inducidas por el ideal $p^{n} \mathbb{Z}$}

Como ya hemos mencionado, primero trabajaremos específicamente con la conexión de Galois entre las retículas de prerradicales $\mathbb{Z}$-pr y $\mathbb{Z}_{p^{n}}$-pr. Para esto, presentamos a continuación, un pequeño resumen de la descripción de las retículas $\mathbb{Z}_{p^{n} \text {-pr. Para más detalles ver [7]. }}$

Recordemos que la retícula de ideales del anillo $\mathbb{Z}_{p^{n}}$ es la cadena finita:

$$
0=I_{0}<I_{1}<\ldots<I_{n}=\mathbb{Z}_{p^{n}},
$$

donde $I_{i}=p^{n-i} \mathbb{Z}_{p^{n}}$, para $i=1, \ldots, n$. Tales ideales son los $\mathbb{Z}_{p^{n} \text {-submódulos }}$ totalmente invariantes de $\mathbb{Z}_{p^{n}}$. Puesto que $\mathbb{Z}_{p^{n}}$ es un anillo local uniserial, por el

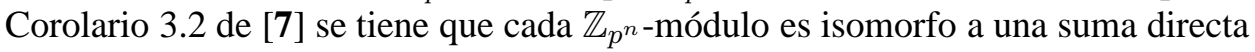
de ideales de $\mathbb{Z}_{p^{n}}$ y por el comportamiento de los prerradicales con respecto a la suma directa, vista en la Proposición 1.47, cada prerradical sobre $\mathbb{Z}_{p^{n}}$ está determinado por su valor en los ideales $I_{k}$ de $\mathbb{Z}_{p^{n}}$. En la proposición 3.1 de [7], la cual enunciaremos enseguida se puede ver la descripción de los prerradicales sobre $\mathbb{Z}_{p^{n}}$ en los ideales $I_{k}$.

Proposición 5.1. Sea $R$ un anillo local uniserial y sea $\sigma \in R$-pr. Sea $k \in\{0, \ldots, n-1\}$. Si $\sigma\left(I_{k}\right)=I_{r}$ para alguna $r \in\{0, \ldots, k\}$, entonces $\sigma\left(I_{k+1}\right)=$ $I_{r} \circ \sigma\left(I_{k+1}\right)=I_{r+1}$. 
Por el Teorema 1.58 y la Proposición 1.59 cada prerradical sobre $\mathbb{Z}_{p^{n}}$ es un

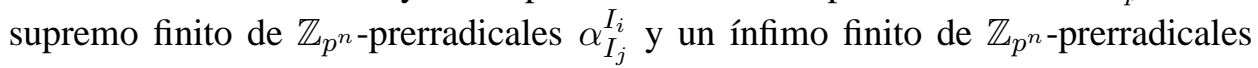
$\omega_{I_{j}}^{I_{i}}$. En lo sucesivo para simplificar la notación usaremos $\alpha_{j}^{i}$, $\omega_{j}^{i}$ en lugar de $\alpha_{I_{j}}^{I_{i}}$ y $\omega_{I_{j}}^{I_{i}}$, para $0 \leq j \leq i \leq n$, respectivamente.

También, como una consecuencia de la Proposición 5.1, existe un isomorfismo

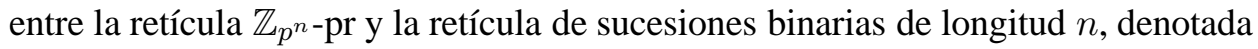
por $B_{n}$, como lo indica la Proposición 4.1 de [7]. Por lo que podemos identificar a los prerradicales sobre $\mathbb{Z}_{p^{n}}$ como $n$-adas de ceros y unos por medio de este

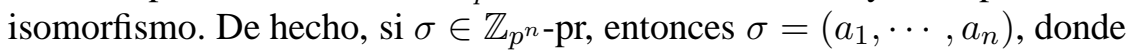

$$
a_{k}=\left\{\begin{array}{l}
0, \text { if } \sigma\left(I_{k}\right)=\sigma\left(I_{k-1}\right), \\
1, \text { if } \sigma\left(I_{k}\right) \neq \sigma\left(I_{k-1}\right) .
\end{array}\right.
$$

\section{Notación.}

1. En adelante, para referirnos a los $\mathbb{Z}_{p^{n} \text {-prerradicales usaremos su corres- }}$ pondiente sucesión binaria.

2. Denotaremos al elemento mayor y menor en $\mathbb{Z}_{p^{n}}$-pr respectivamente como $1_{n}$ y $0_{n}$.

En virtud de la Observación 1.57, con respecto a los prerradicales alfa y omega, la siguiente figura tiene sentido y muestra la correspondiente sucesión binaria para los prerradicales alfa y omega en $\mathbb{Z}_{p^{n} \text {-pr. }}$

\begin{tabular}{|c|c|}
\hline$\alpha_{j}^{i}$ & $\omega_{j}^{i}$ \\
\hline$(\underbrace{0, \ldots 0}_{i-j}, \underbrace{1, \ldots 1}_{j}, \underbrace{0, \ldots 0}_{n-i})$ & $(\underbrace{1, \ldots 1}_{j}, \underbrace{0, \ldots 0}_{i-j}, \underbrace{1, \ldots 1}_{n-i})$ \\
\hline
\end{tabular}

FIGURA 1. Prerradicales alfa y omega sobre $\mathbb{Z}_{p^{n}-\mathrm{pr}}$

Los siguientes casos particulares se pueden ver en las Proposiciones 6.2 y 6.3 de [7]. Los prerradicales idempotentes en $\mathbb{Z}_{p^{n}}$-pr, son de la forma $\alpha_{i}^{i}$ y su correspondiente sucesión binaria consta de un segmento inicial de 1's, quizá vacío, seguido de un segmento de 0's, que podría ser vacío. Dualmente los radicales so-

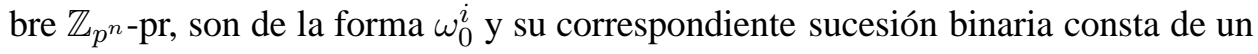
segmento inicial de 0's, quizá vacío, seguido de un segmento de 1's, que podría ser vacío. Por lo tanto los únicos prerradicales radicales idempotentes sobre $\mathbb{Z}_{p^{n}}$, son $1_{n}$ y $0_{n}$.

Ejemplo 5.2. Por [7], la retícula $\mathbb{Z}_{p^{3}}$-pr es como en la Figura 2. Los prerradicales idempotentes son $(0,0,0),(1,0,0),(1,1,0)$ y $(1,1,1)$. Dualmente los radicales son $(1,1,1),(0,1,1),(0,0,1) y(0,0,0)$. 


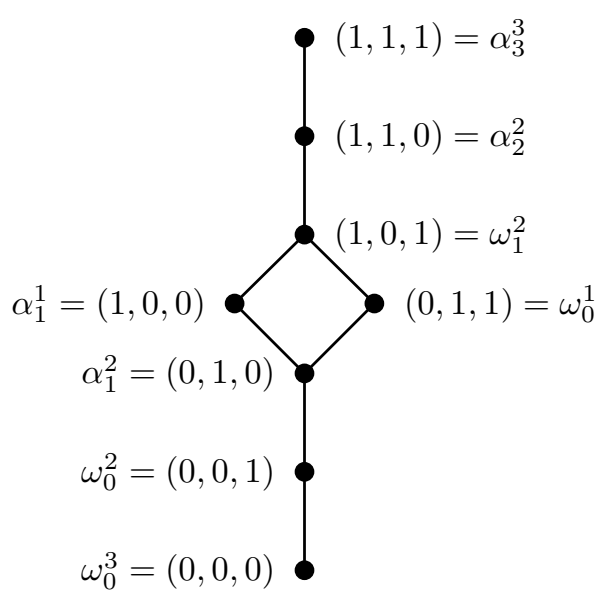

FIGURA 2. La retícula $\mathbb{Z}_{p^{3}-\mathrm{pr}}$

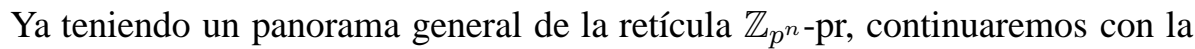
descripción de los intervalos en $\mathbb{Z}$-pr inducidos por las imágenes inversas bajo el morfismo $\varphi_{I}$ de la conexión de Galois entre las retículas $\mathbb{Z}$-pr y $\mathbb{Z}_{p^{n}}$-pr.

Proposición 5.3. Sea $\mathcal{C}_{p}^{n}=\left\{\mathbb{Z}_{p^{i}} \mid i=1, \cdots, n\right\} \subseteq \mathbb{Z}$-Mod y $\tau \in \mathbb{Z}$-pr. Entonces

$$
[\tau]_{I}=[\tau]_{p^{n} \mathbb{Z}}=[\tau]_{\mathcal{C}_{p}^{n}}
$$

Observe que $\mathcal{C}_{p}^{n}$ es un conjunto de grupos cíclicos finitos.

Demostración. Sea $\tau \in \mathbb{Z}$-pr. Por la Proposición 1.87, se tiene que $[\tau]_{p^{n} \mathbb{Z}}=$ $\left[\bigvee_{M \in \mathcal{C}_{I}} \alpha_{\tau M}^{M}, \bigwedge_{M \in \mathcal{C}_{I}} \omega_{\tau M}^{M}\right]$. Sea $\sigma \in[\tau]_{p^{n}} \mathbb{Z}$. Entonces $\sigma\left(\mathbb{Z}_{p^{i}}\right)=\tau\left(\mathbb{Z}_{p^{i}}\right)$ para $i=$ $1, \cdots, n$, puesto que un ideal de $\mathbb{Z}_{p^{n}}$ es isomorfo como $\mathbb{Z}$-módulo a un elemento $\mathcal{C}_{p}^{n}$. De ahí que $\sigma \in[\tau]_{\mathcal{C}_{p}^{n}}$.

Por otro lado sea $\sigma \in[\tau]_{\mathcal{C}_{p}^{n}}$ y $M \in \mathcal{C}_{I}$. Por [7], sabemos que $\mathbb{Z}_{p^{n}}$ es un anillo local uniserial y por Corolario 3.2 en [7], se tiene que $M=\bigoplus_{k=1}^{m} N_{k}$, donde $m \leq n$ y $N_{k} \cong I_{k}$ para algún $I_{k}$ ideal de $\mathbb{Z}_{p^{n}}$. Puesto que los ideales de $\mathbb{Z}_{p^{n}}$ son isomorfos como $\mathbb{Z}$-módulos a los grupos cíclicos finitos, se sigue que $N_{k} \in \mathcal{C}_{p}^{n}$. Entonces, por la Proposición 1.47, parte 1:

$$
\sigma(M)=\bigoplus_{k=1}^{m} \sigma\left(N_{k}\right)=\bigoplus_{k=1}^{m} \tau\left(N_{k}\right)=\tau\left(\bigoplus_{k=1}^{m} N_{k}\right)=\tau M
$$

es decir, $\sigma \in[\tau]_{p^{n} \mathbb{Z}}$. Por lo tanto $[\tau]_{p^{n} \mathbb{Z}}=[\tau]_{\mathcal{C}_{p}^{n}}$. 
Por cuestión de notación, de ahora en adelante cuando hablemos de la clase de equivalencia de $\tau \in \mathbb{Z}$-pr con respecto a la clase $\mathcal{C}_{p}^{n}$, usaremos la siguiente notación

$$
[\tau]_{\mathcal{C}_{p}^{n}}=[\tau]_{p}^{n}
$$

Por lo tanto en este caso, podemos describir los intervalos en $\mathbb{Z}$-pr inducidos por las imágenes inversas bajo el morfismo $\varphi_{I}$, que depende del ideal $I=p^{n} \mathbb{Z}$, de la forma siguiente.

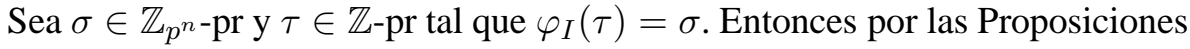
4.21 y 5.3 tenemos

$$
\begin{aligned}
\overleftarrow{\varphi_{I}}(\sigma) & =[\tau]_{I}=[\tau]_{p}^{n}=\left[\bigvee_{\mathbb{Z}_{p^{i}} \in \mathcal{C}_{p}^{n}} \alpha_{\tau \mathbb{Z}_{p^{i}}}^{\mathbb{Z}_{p^{i}}}, \bigwedge_{\mathbb{Z}_{p^{i}} \in \mathcal{C}_{p}^{n}} \omega_{\tau \mathbb{Z}_{p^{i}}}^{\mathbb{Z}_{p^{i}}}\right] \\
& =\left[\bigvee_{i=1}^{n} \alpha_{\tau \mathbb{Z}_{p^{i}}}^{\mathbb{Z}_{p^{i}}}, \bigwedge_{i=1}^{n} \omega_{\tau \mathbb{Z}_{p^{i}}}^{\mathbb{Z}_{p^{i}}}\right] \\
& =\left[\bigvee_{i=1}^{n} \alpha_{\sigma \mathbb{Z}_{p^{i}}}^{\mathbb{Z}_{p^{i}}}, \bigwedge_{i=1}^{n} \omega_{\sigma \mathbb{Z}_{p^{i}}}^{\mathbb{Z}_{p^{i}}}\right]
\end{aligned}
$$

Ya que $\varphi_{I}(\tau)=\sigma$, por definición del morfismo $\varphi_{I}$, el prerradical $\sigma$ es la restricción

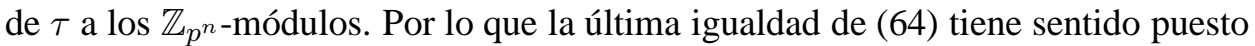
que el valor de $\tau$ en los $\mathbb{Z}$-módulos $\mathbb{Z}_{p^{r}}$ es el mismo que el de $\sigma$ en los ideales $I_{r}$ de $\mathbb{Z}_{p^{n}}$. En particular, los $\mathbb{Z}$-prerradicales $\alpha_{\mathbb{Z}_{p^{j}}}^{\mathbb{Z}_{p^{i}}}$ y $\omega_{\mathbb{Z}_{p^{j}}}^{\mathbb{Z}_{p^{i}}}$ se pueden evaluar en los módulos $\mathbb{Z}_{p^{r}}$, a partir, de la correspondiente sucesión binaria vista en la Figura 1.

Así, esta descripción de los intervalos en $\mathbb{Z}$-pr, facilita el cálculo para hallarlos,

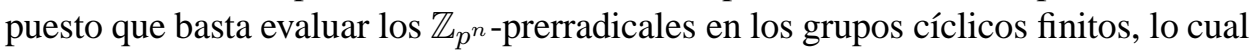
podemos consultar en [7].

\subsubsection{Intervalos en $\mathbb{Z}$-pr inducidos por prerradicales idem- potentes y radicales en $\mathbb{Z}_{p^{n}}$-pr}

Usando la información de las Proposiciones 6.2 y 6.3 de [7] y el último párrafo previo a esta sección, ahora analizaremos las imágenes inversas bajo $\varphi_{I}$ de los prerradicales idempotentes y los radicales sobre $\mathbb{Z}_{p^{n}}$.

El siguiente lema muestra cómo son los $\mathbb{Z}$-prerradicales alfa y omega evaluados en ciertos grupos cíclicos finitos, esto nos servirá para estudiar las imágenes inversas bajo $\varphi_{I}$ de los prerradicales que nos interesa en esta sección.

Lema 5.4. Sea $p$ un número primo y sean $i, j, r \geq 1$ tal que $i>j$. Entonces

$$
\text { 1. } \alpha_{\mathbb{Z}_{p^{j}}}^{\mathbb{Z}_{p^{i}}}\left(\mathbb{Z}_{p^{r}}\right)=\left\{\begin{array}{l}
0 \text { si } 1 \leq r \leq i-j \\
\mathbb{Z}_{p^{r-(i-j)}} \text { si } i-j<r \leq i \\
\mathbb{Z}_{p^{j}} \text { si } r>i
\end{array}\right.
$$


2. $\omega_{\mathbb{Z}_{p^{j}}}^{\mathbb{Z}_{p^{i}}}\left(\mathbb{Z}_{p^{r}}\right)=\left\{\begin{array}{l}\mathbb{Z}_{p^{r}} \text { si } 1 \leq r \leq j \\ \mathbb{Z}_{p^{j}} \text { si } j<r \leq i \\ \mathbb{Z}_{p^{r-(i-j)} \text { if } r>i}\end{array}\right.$

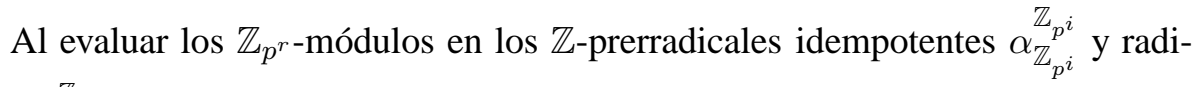
cales $\omega_{0}^{\mathbb{Z}_{p}{ }^{i}}$, se obtiene el siguiente lema.

Lema 5.5. Sea $p$ un número primo y sean $i, r \geq 1$. Entonces

1. $\alpha_{\mathbb{Z}_{p^{i}}}^{\mathbb{Z}_{p^{i}}}\left(\mathbb{Z}_{p^{r}}\right)=\left\{\begin{array}{l}\mathbb{Z}_{p^{r}} \text { if } 1 \leq r \leq i \\ \mathbb{Z}_{p^{i}} \text { if } r>i\end{array}\right.$ 2. $\omega_{0}^{\mathbb{Z}_{p^{i}}}\left(\mathbb{Z}_{p^{r}}\right)=\left\{\begin{array}{l}0 \text { si } 1 \leq r \leq i \\ \mathbb{Z}_{p^{r-i}} \text { si } r>i\end{array}\right.$

Los submódulos de los $\mathbb{Z}$-módulos cíclicos son totalmente invariantes, por lo que de la Observación 1.57 y los Lemas 5.4 y 5.5, se tiene el siguiente resultado que describe ciertas cadenas de $\mathbb{Z}$-prerradicales.

Corolario 5.6. Sea p un número primo. Entonces se tiene las siguientes cadenas de $\mathbb{Z}$-prerradicales:

1. $\alpha_{\mathbb{Z}_{p}}^{\mathbb{Z}_{p}} \prec \alpha_{\mathbb{Z}_{p^{2}}}^{\mathbb{Z}_{p^{2}}} \prec \cdots \prec \alpha_{\mathbb{Z}_{p^{i}}}^{\mathbb{Z}_{p^{i}}} \prec \cdots$

2. $\alpha_{\mathbb{Z}_{p}}^{\mathbb{Z}_{p^{i+1}}} \prec \alpha_{\mathbb{Z}_{p^{2}}}^{\mathbb{Z}^{i+2}} \prec \cdots \prec \alpha_{\mathbb{Z}_{p^{j}}}^{\mathbb{Z}_{p^{i+j}}} \prec \cdots$ para $i \geq 1$.

3. $\alpha_{\mathbb{Z}_{p^{i}}}^{\mathbb{Z}_{p^{i}}} \succ \alpha_{\mathbb{Z}_{p^{i}}}^{\mathbb{Z}_{p^{i+1}}} \succ \cdots \succ \alpha_{\mathbb{Z}_{p^{i}}}^{\mathbb{Z}_{p^{i+j}}} \succ \cdots$ para $i \geq 1$.

4. $\omega_{0}^{\mathbb{Z}_{p}} \succ \omega_{0}^{\mathbb{Z}_{p^{2}}} \succ \cdots \succ \omega_{0}^{\mathbb{Z}_{p^{i}}} \succ \cdots$

5. $\omega_{\mathbb{Z}_{p^{i}}}^{\mathbb{Z}_{p^{i+1}}} \succ \omega_{\mathbb{Z}_{p^{i}}}^{\mathbb{Z}_{p^{i+2}}} \succ \cdots \succ \omega_{\mathbb{Z}_{p^{i}}}^{\mathbb{Z}_{p^{i+j}}} \succ \cdots$ para $i \geq 1$.

6. $\omega_{0}^{\mathbb{Z}_{p^{i}}} \prec \omega_{\mathbb{Z}_{p}}^{\mathbb{Z}_{p^{i+1}}} \prec \omega_{\mathbb{Z}_{p^{2}}}^{\mathbb{Z}_{p^{i+2}}} \prec \cdots \prec \omega_{\mathbb{Z}_{p^{j}}}^{\mathbb{Z}_{p^{i+j}}} \prec \cdots$ para $i \geq 1$.

Demostración. 1. Por el Lema 5.5, para $i \geq 1, \alpha_{\mathbb{Z}_{p^{i+1}}}^{\mathbb{Z}_{p^{i+1}}}\left(\mathbb{Z}_{p^{i}}\right)=\mathbb{Z}_{p^{i}}$. Por lo que $\alpha_{\mathbb{Z}_{p^{i}}}^{\mathbb{Z}_{p^{i}}} \prec \alpha_{\mathbb{Z}_{p^{i+1}}}^{\mathbb{Z}_{p^{i+1}}} \cdot$

2. Por el Lema 5.4, para $i, j \geq 1, \alpha_{\mathbb{Z}_{p^{j+1}}}^{\mathbb{Z}_{p^{i+j+1}}}\left(\mathbb{Z}_{p^{i+j}}\right)=\mathbb{Z}_{p^{j}}$. Así que $\alpha_{\mathbb{Z}_{p^{j}}}^{\mathbb{Z}_{p^{i+j}}} \prec$ $\alpha_{\mathbb{Z}_{p^{j+1}}}^{\mathbb{Z}_{p^{i+j+1}}}$.

3. Por el Lema 5.4, para $i \geq 1$ y $j \geq 0, \alpha_{\mathbb{Z}_{p^{i}}}^{\mathbb{Z}_{p^{i+j}}}\left(\mathbb{Z}_{p^{i+j+1}}\right)=\mathbb{Z}_{p^{i}}$. Por lo tanto $\alpha_{\mathbb{Z}_{p^{i}}}^{\mathbb{Z}_{p^{i+j+1}}} \prec \alpha_{\mathbb{Z}_{p^{i}}}^{\mathbb{Z}_{p^{i+j}}}$

4. Por el Lema 5.5, para $i \geq 1, \omega_{0}^{\mathbb{Z}_{p^{i+1}}}\left(\mathbb{Z}_{p^{i}}\right)=0$. Por lo que $\omega_{0}^{\mathbb{Z}_{p^{i+1}}} \prec \omega_{0}^{\mathbb{Z}_{p^{i}}}$.

5. Por el Lema 5.4, para $i \geq 1$ y $j \geq 0, \omega_{\mathbb{Z}_{p^{i}}}^{\mathbb{Z}_{p^{i+j+1}}}\left(\mathbb{Z}_{p^{i+j}}\right)=\mathbb{Z}_{p^{i}}$. Así, $\omega_{\mathbb{Z}_{p^{i}}}^{\mathbb{Z}_{p^{i+j+1}}} \prec \omega_{\mathbb{Z}_{p^{i}}}^{\mathbb{Z}_{p^{i+j}}}$ 
6. Por el Lema 5.5, para $i \geq 1, \omega_{0}^{\mathbb{Z}_{p^{i}}}\left(\mathbb{Z}_{p^{i+1}}\right)=\mathbb{Z}_{p}$. Entonces $\omega_{0}^{\mathbb{Z}_{p^{i}}} \prec \omega_{\mathbb{Z}_{p}}^{\mathbb{Z}_{p^{i+1}}}$. Por otro lado, por el Lema 5.4, para $i, j \geq 1, \omega_{\mathbb{Z}_{p^{j}}}^{\mathbb{Z}_{p^{i+j}}}\left(\mathbb{Z}_{p^{i+j+1}}\right)=\mathbb{Z}_{p^{j+1}}$. Por lo que $\omega_{\mathbb{Z}_{p j}}^{\mathbb{Z}_{p^{i+j}}} \prec \omega_{\mathbb{Z}_{p^{j+1}}}^{\mathbb{Z}_{p^{i+j+1}}}$

Veamos la siguiente figura que muestra las cadenas del Corolario 5.6.
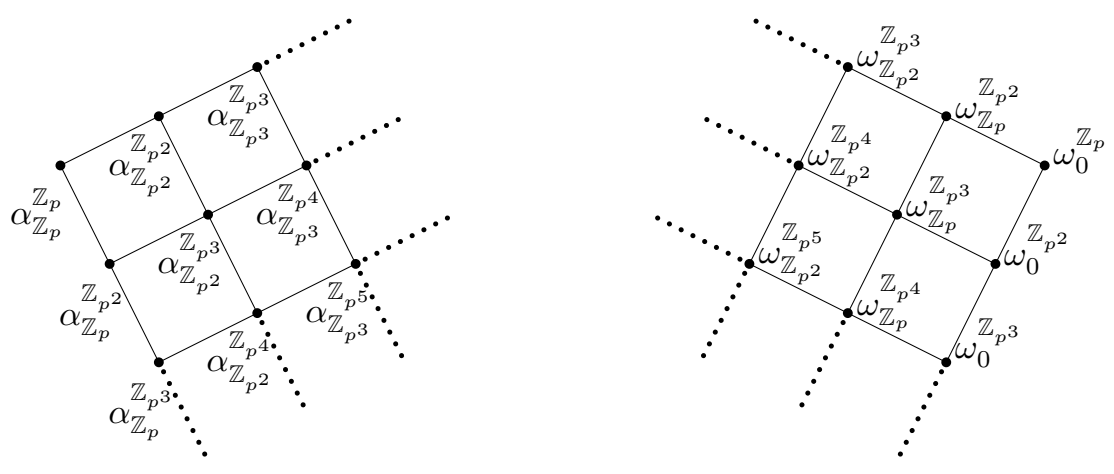

FIGURA 3. Los copos $\left\{\alpha_{\mathbb{Z}_{p^{j}}}^{\mathbb{Z}_{p^{i}}}\right\}_{1 \leq j \leq i}$ y $\left\{\omega_{\mathbb{Z}_{p^{j}}}^{\mathbb{Z}_{p^{i}}}\right\}_{1 \leq j \leq i} \cup\left\{\omega_{0}^{\mathbb{Z}_{p^{i}}}\right\}_{1 \leq i}$

Ahora sí, describiremos los intervalos $\overleftarrow{\varphi_{I}}(\sigma)$ inducidos por un prerradical idempotente $\sigma$ sobre $\mathbb{Z}_{p^{n}}$.

Proposición 5.7. Sea $\sigma \in \mathbb{Z}_{p^{n}-p r}$ un prerradical idempotente, tal que $\sigma \neq 0$. Entonces $\overleftarrow{\varphi_{I}}(\sigma)=\left[\alpha_{\mathbb{Z}_{p}^{m}}^{\mathbb{Z}_{p^{m}}}, \omega_{\mathbb{Z}_{p^{m}}}^{\mathbb{Z}^{n}}\right]$, donde $1 \leq m \leq n$ y $m$ es la longitud del segmento inicial con entradas el número 1 de la sucesión binaria que representa a $\sigma$.

Demostración. Por (64), tenemos:

$$
\begin{aligned}
\overleftarrow{\varphi_{I}}(\sigma) & =\left[\bigvee_{i=1}^{m} \alpha_{\mathbb{Z}_{p^{i}}}^{\mathbb{Z}_{p^{i}}} \vee \bigvee_{i=m+1}^{n} \alpha_{\mathbb{Z}_{p^{m}}}^{\mathbb{Z}_{p^{i}}}, \bigwedge_{i=1}^{m} \omega_{\mathbb{Z}_{p^{i}}}^{\mathbb{Z}_{p^{i}}} \wedge \bigwedge_{i=m+1}^{n} \omega_{\mathbb{Z}_{p^{m}}}^{\mathbb{Z}_{p^{i}}}\right] \\
& =\left[\bigvee_{i=1}^{m} \alpha_{\mathbb{Z}_{p^{i}}}^{\mathbb{Z}_{p^{i}}} \vee \bigvee_{i=m+1}^{n} \alpha_{\mathbb{Z}_{p^{m}}}^{\mathbb{Z}_{p^{i}}}, \bigwedge_{i=m+1}^{n} \omega_{\mathbb{Z}_{p^{m}}}^{\mathbb{Z}_{i}}\right] \\
& =\left[\alpha_{\mathbb{Z}_{p} m}^{\mathbb{Z}_{p^{m}}}, \omega_{\mathbb{Z}_{p^{m}}}^{\mathbb{Z}_{p^{n}}}\right]
\end{aligned}
$$

puesto que por el Corolario 5.6, se tiene lo siguiente:

$$
\begin{aligned}
& \bigvee_{i=1}^{m} \alpha_{\mathbb{Z}_{p^{i}}}^{\mathbb{Z}_{p^{i}}}=\alpha_{\mathbb{Z}_{p} m}^{\mathbb{Z}_{p^{m}}}, \bigvee_{i=m+1}^{n} \alpha_{\mathbb{Z}_{p^{m}}}^{\mathbb{Z}_{p^{i}}}=\alpha_{\mathbb{Z}_{p}}^{\mathbb{Z}_{p^{m+1}}}, \alpha_{\mathbb{Z}_{p} m}^{\mathbb{Z}_{p^{m}}} \vee \alpha_{\mathbb{Z}_{p} m}^{\mathbb{Z}_{p^{m+1}}}=\alpha_{\mathbb{Z}_{p} m}^{\mathbb{Z}_{p^{m}}} \mathrm{y} \\
& \bigwedge_{i=m+1}^{n} \omega_{\mathbb{Z}_{p^{m}}}^{\mathbb{Z}_{p^{i}}}=\omega_{\mathbb{Z}_{p^{m}}}^{\mathbb{Z}_{p^{n}}}
\end{aligned}
$$


Corolario 5.8. $\overleftarrow{\varphi_{I}}\left(1_{n}\right)=\left[\alpha_{\mathbb{Z}_{p^{n}}}^{\mathbb{Z}_{p^{n}}}, 1_{\mathbb{Z}}\right]$

Demostración. El resultado se sigue de la Proposición 5.7 para $m=n$, puesto que

$$
\overleftarrow{\varphi_{I}}\left(1_{n}\right)=\left[\alpha_{\mathbb{Z}_{p^{n}}}^{\mathbb{Z}_{p^{n}}}, \omega_{\mathbb{Z}_{p^{n}}}^{\mathbb{Z}_{p^{n}}}\right]=\left[\alpha_{\mathbb{Z}_{p^{n}}}^{\mathbb{Z}_{p^{n}}}, 1_{\mathbb{Z}}\right]
$$

Observemos que de los Corolarios 4.18 y 5.8, tenemos

$$
\left[t\left(\alpha_{p^{n} \mathbb{Z}}^{\mathbb{Z}}\right), 1_{\mathbb{Z}}\right]=\overleftarrow{\varphi_{I}}\left(1_{n}\right)=\left[\alpha_{\mathbb{Z}_{p^{n}}}^{\mathbb{Z}_{p^{n}}}, 1_{\mathbb{Z}}\right]
$$

por lo que,

$$
t\left(\alpha_{p^{n} \mathbb{Z}}^{\mathbb{Z}}\right)=\alpha_{\mathbb{Z}_{p^{n}}}^{\mathbb{Z}_{p^{n}}}
$$

que confirma lo que ya habíamos comentado.

Dualmente, el siguiente resultado describe los intervalos $\overleftarrow{\varphi_{I}}(\sigma)$ inducidos por

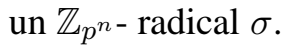

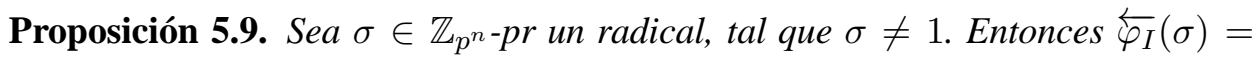
$\left[\alpha_{\mathbb{Z}_{p^{n-k}}}^{\mathbb{Z}_{p^{n}}}, \omega_{0}^{\mathbb{Z}_{p^{k}}}\right]$, donde $1 \leq k<n$ y $k$ es la longitud del segmento inicial con entradas el número 0 de la sucesión binaria que representa a $\sigma$.

Demostración. Por (64), tenemos:

$$
\begin{aligned}
\overleftarrow{\varphi_{I}}(\sigma) & =\left[\bigvee_{i=1}^{k} \alpha_{0}^{\mathbb{Z}_{p^{i}}} \vee \bigvee_{i=k+1}^{n} \alpha_{\mathbb{Z}_{p^{i-k}}}^{\mathbb{Z}_{p^{i}}}, \bigwedge_{i=1}^{k} \omega_{0}^{\mathbb{Z}_{p^{i}}} \wedge \bigwedge_{i=k+1}^{n} \omega_{\mathbb{Z}_{p^{i-k}}}^{\mathbb{Z}_{p^{i}}}\right] \\
& =\left[\bigvee_{i=k+1}^{n} \alpha_{\mathbb{Z}_{p^{i}}}^{\mathbb{Z}_{p^{i}}}, \bigwedge_{i=1}^{k} \omega_{0}^{\mathbb{Z}_{p^{i}}} \wedge \bigwedge_{i=k+1}^{n} \omega_{\mathbb{Z}_{p^{i}}}^{\mathbb{Z}_{p^{i}}}\right] \\
& =\left[\alpha_{\mathbb{Z}_{p^{n-k}}}^{\mathbb{Z}^{n}}, \omega_{0}^{\mathbb{Z}_{p^{k}}}\right]
\end{aligned}
$$

ya que por el Corolario 5.6, obtenemos $\bigvee_{i=k+1}^{n} \frac{\mathbb{Z}_{p^{i}}}{\alpha_{p^{i-k}}}=\alpha_{\mathbb{Z}_{p^{n-k}}}^{\mathbb{Z}_{p^{n}}}$,

$$
\bigwedge_{i=1}^{k} \omega_{0}^{\mathbb{Z}_{p^{i}}}=\omega_{0}^{\mathbb{Z}_{p^{k}}}, \bigwedge_{i=k+1}^{n} \omega_{\mathbb{Z}_{p^{i-k}}}^{\mathbb{Z}_{i}}=\omega_{\mathbb{Z}_{p}}^{\mathbb{Z}_{p^{k+1}}} \mathrm{y} \omega_{0}^{\mathbb{Z}_{p^{k}}} \wedge \omega_{\mathbb{Z}_{p}}^{\mathbb{Z}_{p^{k+1}}}=\omega_{0}^{\mathbb{Z}_{p^{k}}}
$$

La siguiente proposición, es para $k=n$.

Proposición 5.10. $\overleftarrow{\varphi_{I}}\left(0_{n}\right)=\left[0_{\mathbb{Z}}, \omega_{0}^{\mathbb{Z}_{p^{n}}}\right]$.

Demostración. Por la igualdad (64) y el Lema 5.4, tenemos:

$$
\overleftarrow{\varphi_{I}}\left(0_{n}\right)=\left[\bigvee_{i=1}^{n} \alpha_{0}^{\mathbb{Z}_{p^{i}}}, \bigwedge_{i=1}^{n} \omega_{0}^{\mathbb{Z}_{p^{i}}}\right]=\left[0_{\mathbb{Z}}, \bigwedge_{i=1}^{n} \omega_{0}^{\mathbb{Z}_{p^{i}}}\right]=\left[0_{\mathbb{Z}}, \omega_{0}^{\mathbb{Z}_{p^{n}}}\right]
$$


Notemos que por el Corolario 4.17 y la Proposición 5.10, se tiene que

$$
\left[0_{\mathbb{Z}}, \alpha_{p^{n} \mathbb{Z}}^{\mathbb{Z}}\right]=\overleftarrow{\varphi_{I}}\left(0_{n}\right)=\left[0_{\mathbb{Z}}, \omega_{0}^{\mathbb{Z} p^{n}}\right]
$$

es decir,

$$
\alpha_{p^{n} \mathbb{Z}}^{\mathbb{Z}}=\omega_{0}^{\mathbb{Z}_{p^{n}}}
$$

Recuerde de (52), que $\tau_{\eta}=\psi_{I}\left(0_{n}\right)=\alpha_{p^{n} \mathbb{Z}}^{\mathbb{Z}}$ es el menor cerrado en $\mathbb{Z}$-pr.

Los siguientes resultados son consecuencia de las Proposiciones 5.7 y 5.9.

Corolario 5.11. $\overleftarrow{\varphi_{I}}(1,0,0, \cdots, 0)=\left[\alpha_{\mathbb{Z}_{p}}^{\mathbb{Z}_{p}}, \omega_{\mathbb{Z}_{p}}^{\mathbb{Z}_{p^{n}}}\right]$

DEMOSTRACIÓN.

El resultado se sigue de la Proposición 5.7 para $m=1$.

Corolario 5.12. $\overleftarrow{\varphi_{I}}(0,1,1, \cdots, 1)=\left[\alpha_{\mathbb{Z}_{p^{n-1}}}^{\mathbb{Z}_{p^{n}}}, \omega_{0}^{\mathbb{Z}_{p}}\right]$

Demostración. El resultado se tiene de la Proposición 5.9 para $k=1$.

Del Corolario 4.13 se concluye que en $\overleftarrow{\varphi_{I}}(1,0,0, \cdots, 0)$ no hay $\mathbb{Z}$-radicales, del mismo modo por el Corolario 4.12 notamos que en $\overleftarrow{\varphi_{I}}(0,1,1, \cdots, 1)$ no hay $\mathbb{Z}$-prerradicales idempotentes.

Ejemplo 5.13. Sea $I=p^{2} \mathbb{Z}$ un ideal de $\mathbb{Z} y R / I=\mathbb{Z} / p^{2} \mathbb{Z} \cong \mathbb{Z}_{p^{2}}$. En este caso la adjunción $\left\langle F_{I}, G_{I}\right\rangle$ induce la conexión de Galois $\left\langle\mathbb{Z}-p r, \varphi_{I}, \psi_{I}, \mathbb{Z}_{p^{2}}-p r\right\rangle$, donde $\varphi_{I}: \mathbb{Z}$-pr $\rightarrow \mathbb{Z}_{p^{2}}$-pr y $\psi_{I}: \mathbb{Z}_{p^{2}}$-pr $\rightarrow \mathbb{Z}$-pr.

Se tiene que $\mathbb{Z}_{p^{2}}$-pr tiene 4 elementos, con representación en sucesiones binarias, $(0,0),(0,1),(1,0)$ y $(1,1)$. Para describir la partición $\mathcal{P}_{p^{2} \mathbb{Z}}$ de $\mathbb{Z}$-pr inducida por el ideal $p^{2} \mathbb{Z}$, veamos lo siguiente:

1. Por (49) y (50), tenemos que $\psi_{I}\left(1_{\mathbb{Z}_{p^{2}}}\right)=1_{\mathbb{Z}}$ y $\varphi_{I}\left(0_{\mathbb{Z}}\right)=0_{\mathbb{Z}_{p^{2}}}$.

2. Por (51) y (52), respectivamente tenemos que el mayor abierto en $\mathbb{Z}_{p^{2}}-p r$, es $1_{\mathbb{Z}_{p^{2}}}=\varphi_{I}\left(1_{\mathbb{Z}}\right)$ y el menor cerrado en $\mathbb{Z}$-pr es $\alpha_{p^{2} \mathbb{Z}}^{\mathbb{Z}}=\psi_{I}\left(0_{\mathbb{Z}_{p^{2}}}\right)$.

3. Por el Corolario 5.10, se tiene que $\overleftarrow{\varphi_{I}}(0,0)=\left[0_{\mathbb{Z}}, \omega_{0}^{\mathbb{Z}_{p^{2}}}\right]=\left[0_{\mathbb{Z}}, \alpha_{p^{2} \mathbb{Z}}^{\mathbb{Z}}\right]$.

4. Por el Corolario 5.8, obtenemos que $\overleftarrow{\varphi_{I}}(1,1)=\left[\alpha_{\mathbb{Z}_{p^{2}}}^{\mathbb{Z}_{p^{2}}}, 1_{\mathbb{Z}}\right]=\left[t\left(\alpha_{p^{2} \mathbb{Z}}^{\mathbb{Z}}\right), 1_{\mathbb{Z}}\right]$.

5. Por el Corolario 5.11, $\overleftarrow{\varphi_{I}}(1,0)=\left[\alpha_{\mathbb{Z}_{p}}^{\mathbb{Z}_{p}}, \omega_{\mathbb{Z}_{p}}^{\mathbb{Z}_{p^{2}}}\right]$.

6. Por el Corolario 5.12, $\overleftarrow{\varphi_{I}}(0,1)=\left[\alpha_{\mathbb{Z}_{p}}^{\mathbb{Z}_{p^{2}}}, \omega_{0}^{\mathbb{Z}_{p}}\right]$

Por lo tanto $\mathcal{P}_{p^{2} \mathbb{Z}}=\left\{\left[0_{\mathbb{Z}}, \omega_{0}^{\mathbb{Z}_{p^{2}}}\right],\left[\alpha_{\mathbb{Z}_{p}}^{\mathbb{Z}_{p^{2}}}, \omega_{0}^{\mathbb{Z}_{p}}\right],\left[\alpha_{\mathbb{Z}_{p}}^{\mathbb{Z}_{p}}, \omega_{\mathbb{Z}_{p}}^{\mathbb{Z}_{p^{2}}}\right],\left[\alpha_{\mathbb{Z}_{p^{2}}}^{\mathbb{Z}_{p^{2}}}, 1_{\mathbb{Z}}\right]\right\}$.

Con base en lo anterior y al ideal $I=p^{2} \mathbb{Z}$, la partición de $\mathbb{Z}$-pr es como sigue: 


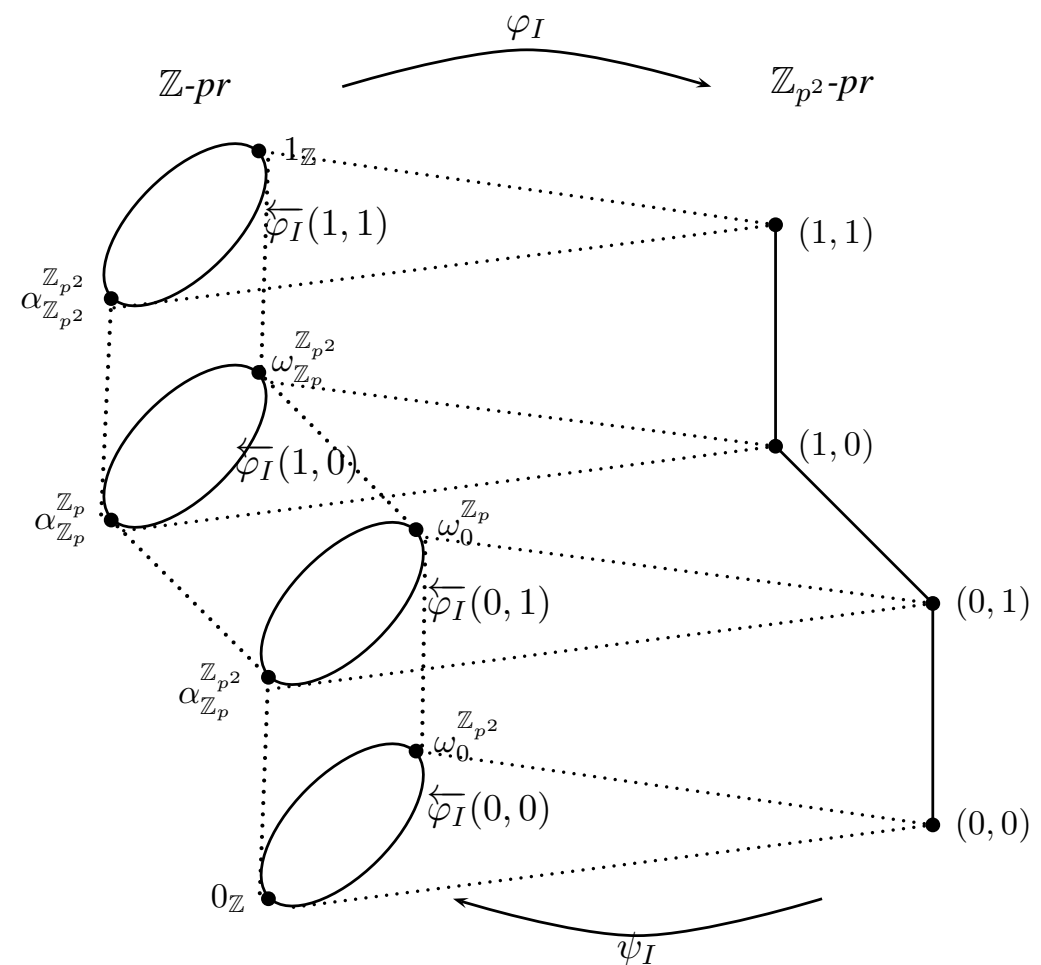

Figura 4. La conexión de Galois $\left\langle\varphi_{I}, \psi_{I}\right\rangle$ para $I=p^{2} \mathbb{Z}$

En vista de los Corolarios 4.12 y 4.13, a continuación mostraremos los intervalos en $\mathbb{Z}$-pr en donde se encuentran algunos $\mathbb{Z}$-perradicales especiales. Dichos intervalos dependen de la partición inducida por las imágenes inversas bajo $\varphi_{I}$, donde $I=p^{n} \mathbb{Z}$ con $p$ primo y $n \geq 1$.

Recordemos del Ejemplo 1.70 que el prerradical Soc(_) es idempotente para cualquier anillo $R$. En $\mathbb{Z}$-pr se tiene que $\operatorname{Soc}\left(\_\right)=\bigvee_{q \text { primo }} \alpha_{\mathbb{Z}_{q}}$. Entonces por (64) tenemos que:

$$
\left[\operatorname{Soc}\left(\_\right)\right]_{p}^{n}=\left[\bigvee_{i=1}^{n} \alpha_{\operatorname{Soc}\left(\mathbb{Z}_{p^{i}}\right)}^{\mathbb{Z}_{p^{i}}}, \bigwedge_{i=1}^{n} \omega_{\operatorname{Soc}\left(\mathbb{Z}_{p^{i}}\right)}^{\mathbb{Z}_{p^{i}}}\right]
$$

Donde $\operatorname{Soc}\left(\mathbb{Z}_{p^{i}}\right)=\bigvee_{q \text { primo }} \alpha_{\mathbb{Z}_{q}}^{\mathbb{Z}_{q}}\left(\mathbb{Z}_{p^{i}}\right)$ para $i=1, \cdots, n$. Por 1 del Lema 5.5 y puesto que $\operatorname{Hom}\left(\mathbb{Z}_{q}, \mathbb{Z}_{p^{i}}\right)=0$ para $q \neq p$ obtenemos:

$$
\bigvee_{q \text { primo }} \alpha_{\mathbb{Z}_{q}}^{\mathbb{Z}_{q}}\left(\mathbb{Z}_{p^{i}}\right)=\alpha_{\mathbb{Z}_{p}}^{\mathbb{Z}_{p}}\left(\mathbb{Z}_{p^{i}}\right)=\mathbb{Z}_{p}
$$


para toda $i \geq 1$. Por 3 y 5 del Corolario 5.6 y de (67):

$$
\left[\operatorname{Soc}\left(\_\right)\right]_{p}^{n}=\left[\bigvee_{i=1}^{n} \alpha_{\mathbb{Z}_{p}^{p^{i}}}^{\mathbb{Z}^{i}}, \bigwedge_{i=1}^{n} \omega_{\mathbb{Z}^{p}}^{\mathbb{Z}_{p^{i}}}\right]=\left[\alpha_{\mathbb{Z}_{p}}^{\mathbb{Z}_{p}}, \omega_{\mathbb{Z}_{p}}^{\mathbb{Z}_{p^{n}}}\right]
$$

Así que de la igualdad anterior y del Corolario 5.11 concluimos que:

$$
[\operatorname{Soc}(-)]_{p}^{n}=\left[\alpha_{\mathbb{Z}_{p}}^{\mathbb{Z}_{p}}, \omega_{\mathbb{Z}_{p}}^{\mathbb{Z}_{p^{n}}}\right]=\overleftarrow{\varphi_{I}}(1,0,0, \cdots, 0)
$$

para un $p$ primo y $n \geq 1$.

Dualmente, del Ejemplo 1.70, el prerradical Rad(_) es radical para todo anillo $R . \operatorname{En} \mathbb{Z}-$ pr, $\operatorname{Rad}\left(\_\right)=\bigwedge_{q \text { primo }} \omega_{0}^{\mathbb{Z}_{q}}$.

Entonces por (64):

$$
\left[\operatorname{Rad}\left(\_\right)\right]_{p}^{n}=\left[\bigvee_{i=1}^{n} \alpha_{\operatorname{Rad}\left(\mathbb{Z}_{p^{i}}\right)}^{\mathbb{Z}_{p^{i}}}, \bigwedge_{i=1}^{n} \omega_{\operatorname{Rad}\left(\mathbb{Z}_{p^{i}}\right)}^{\mathbb{Z}_{p^{i}}}\right]
$$

$\operatorname{Donde} \operatorname{Rad}\left(\mathbb{Z}_{p^{i}}\right)=\bigwedge_{q \text { primo }} \omega_{0}^{\mathbb{Z}_{q}}\left(\mathbb{Z}_{p^{i}}\right)$ para $i=1, \cdots, n$. Por 2 del Lema 5.5 y puesto que $\operatorname{Hom}\left(\mathbb{Z}_{p^{i}}, \mathbb{Z}_{q}\right)=0$ para $q \neq p$ obtenemos:

$$
\bigwedge_{q \text { primo }} \omega_{0}^{\mathbb{Z}_{q}}\left(\mathbb{Z}_{p^{i}}\right)=\omega_{0}^{\mathbb{Z}_{p}}\left(\mathbb{Z}_{p^{i}}\right)=\left\{\begin{array}{l}
0 \text { si } i=1 \\
\mathbb{Z}_{p^{i-1}} \text { si } i>1
\end{array}\right.
$$

Así que por 2 y 6 del Corolario 5.6 y de (69):

$$
\begin{aligned}
{\left[\operatorname{Rad}\left(\_\right)\right]_{p}^{n} } & =\left[\alpha_{0}^{\mathbb{Z}_{p}} \vee \bigvee_{i=1}^{n-1} \alpha_{\mathbb{Z}_{p^{i}}}^{\mathbb{Z}_{p^{i+1}}}, \omega_{0}^{\mathbb{Z}_{p}} \wedge \bigwedge_{i=1}^{n-1} \omega_{\mathbb{Z}_{p^{i}}}^{\mathbb{Z}_{p^{i+1}}}\right] \\
& =\left[\alpha_{\mathbb{Z}_{p^{n-1}}}^{\mathbb{Z}_{p^{n}}}, \omega_{0}^{\mathbb{Z}_{p}}\right]
\end{aligned}
$$

Por lo anterior y el Corolario 5.12, se concluye que:

$$
\left[\operatorname{Rad}\left(\_\right)\right]_{p}^{n}=\left[\alpha_{\mathbb{Z}_{p^{n-1}}}^{\mathbb{Z}_{p^{n}}}, \omega_{0}^{\mathbb{Z}_{p}}\right]=\overleftarrow{\varphi_{I}}(0,1,1, \cdots, 1)
$$

para un $p$ primo y $n \geq 1$.

También podemos hallar en $\mathbb{Z}$-pr el intervalo en donde se encuentra la torsión usal T(_) : $\mathbb{Z}$-Mod $\rightarrow \mathbb{Z}$-Mod, donde $\mathrm{T}(G)=\{x \in G \mid o(x)<\infty\}$. Se sabe que $\mathrm{T}\left(\_\right)$es un radical idempotente. 5.8:

Se tiene que $\alpha_{\mathbb{Z}_{p^{n}}}^{\mathbb{Z}_{p^{n}}}<\mathrm{T}($ _ $)$, puesto que $\mathrm{T}\left(\mathbb{Z}_{p^{n}}\right)=\mathbb{Z}_{p^{n}}$. De ahí y del Corolario

$$
\mathrm{T}\left(\_\right) \in\left[\alpha_{\mathbb{Z}_{p^{n}}}^{\mathbb{Z}_{p^{n}}}, 1_{\mathbb{Z}}\right]=\overleftarrow{\varphi}_{I}\left(1_{n}\right)
$$

Nótese que (71) también se puede concluir siguiendo (64) del mismo modo que para los prerradicales $\operatorname{Soc}\left(\_\right)$y $\operatorname{Rad}\left(\_\right)$sobre $\mathbb{Z}$. 
De la misma manera, hallaremos los intervalos en $\mathbb{Z}$-pr donde se encuentran los átomos y coátomos sobre $\mathbb{Z}$.

Por el Teorema 1.60, los átomos de $\mathbb{Z}$-pr son:

$$
\left\{\alpha_{\mathbb{Z}_{q}}^{\mathbb{Z}_{q^{\infty}}} \mid q \text { es primo }\right\} \text {. }
$$

El siguiente lema es importante para hallar el intervalo en $\mathbb{Z}$-pr que contiene a los átomos.

Lema 5.14. Sean $q$, p primos. Entonces $\operatorname{Hom}\left(\mathbb{Z}_{q^{\infty}}, \mathbb{Z}_{p^{n}}\right)=0$.

Demostración. Si $q \neq p$, entonces $\operatorname{Hom}\left(\mathbb{Z}_{q^{\infty}}, \mathbb{Z}_{p^{n}}\right)=0$, puesto que todos los elementos de $\mathbb{Z}_{q^{\infty}}$ tienen orden una potencia de $q$ y los elementos de $\mathbb{Z}_{p^{n}}$ tienen orden una potencia de $p$. Por otro lado, sea $q=p$ y supongamos que existe un morfismo $f: \mathbb{Z}_{p^{\infty}} \rightarrow \mathbb{Z}_{p^{n}}$ tal que $f$ no es el morfismo cero. Entonces ker $f$ es un subgrupo propio de $\mathbb{Z}_{p^{\infty}}$, por lo que existe $i \geq 0$ tal que $\operatorname{ker} f=H_{p^{i}}$, donde $H_{p^{i}}=$ $\left\langle\frac{1}{p^{2}}+\mathbb{Z}\right\rangle$. Es conocido que $\mathbb{Z}_{p^{\infty}} / H_{p^{k}} \cong \mathbb{Z}_{p^{\infty}}$ para toda $k \geq 0$. Así, por el primer teorema del isomorfismo para grupos $\mathbb{Z}_{p^{\infty}} \cong \mathbb{Z}_{p^{\infty}} / \operatorname{ker} f \cong \mathbb{Z}_{p^{j}}$ para algún $j=$ $1, \ldots, n$, lo cual no puede suceder. Por lo tanto, en ambos casos $\operatorname{Hom}\left(\mathbb{Z}_{q^{\infty}}, \mathbb{Z}_{p^{n}}\right)=$ 0 .

Del Lema 5.14 y puesto que $\alpha_{\mathbb{Z}_{q}}^{\mathbb{Z}^{\infty}}\left(\mathbb{Z}_{p^{n}}\right)=\sum\left\{f\left(\mathbb{Z}_{q}\right) \mid f: \mathbb{Z}_{q^{\infty}} \rightarrow \mathbb{Z}_{p^{n}}\right\}$ :

$$
\alpha_{\mathbb{Z}_{q}}^{\mathbb{Z}_{q^{\infty}}}\left(\mathbb{Z}_{p^{n}}\right)=0
$$

De donde $\alpha_{\mathbb{Z}_{q}}^{\mathbb{Z}_{q}}<\omega_{0}^{\mathbb{Z}_{p^{n}}}$. Por el Corolario 5.10, se concluye que:

$$
\left\{\alpha_{\mathbb{Z}_{q}}^{\mathbb{Z}_{q} \infty} \mid q \text { es primo }\right\} \subset\left[0_{\mathbb{Z}}, \omega_{0}^{\mathbb{Z}_{p^{n}}}\right]=\overleftarrow{\varphi_{I}}\left(0_{n}\right)
$$

Por otro lado, del Teorema 1.60, los coátomos en $\mathbb{Z}$-pr son:

$$
\left\{\omega_{q \mathbb{Z}}^{\mathbb{Z}} \mid q \text { es primo }\right\} \text {. }
$$

Se tiene que $\omega_{q \mathbb{Z}}^{\mathbb{Z}}\left(\mathbb{Z}_{p^{n}}\right)=\bigcap\left\{\overleftarrow{g}(q \mathbb{Z}) \mid g: \mathbb{Z}_{p^{n}} \rightarrow \mathbb{Z}\right\}$. Es claro que Hom $\left(\mathbb{Z}_{p^{n}}, \mathbb{Z}\right)=$ 0 . Así que $\overleftarrow{g}(q \mathbb{Z})=\left\{x \in \mathbb{Z}_{p^{n}} \mid 0=g(x) \in q \mathbb{Z}\right\}=\mathbb{Z}_{p^{n}}$ y por tanto

$$
\omega_{q \mathbb{Z}}^{\mathbb{Z}}\left(\mathbb{Z}_{p^{n}}\right)=\mathbb{Z}_{p^{n}}
$$

De ahí que $\alpha_{\mathbb{Z}_{p^{n}}}^{\mathbb{Z}_{p^{n}}}<\omega_{q \mathbb{Z}}^{\mathbb{Z}}$ y por el Corolario 5.8, concluimos que:

$$
\left\{\omega_{q \mathbb{Z}}^{\mathbb{Z}} \mid q \text { es primo }\right\} \subset\left[\alpha_{\mathbb{Z}_{p^{n}}}^{\mathbb{Z}_{p^{n}}}, 1_{\mathbb{Z}}\right]=\overleftarrow{\varphi_{I}}\left(1_{n}\right)
$$

Observamos que (72) y (73) se pueden obtener usando (64).

Para finalizar esta sección, en seguida mostraremos de manera particular los intervalos en $\mathbb{Z}$-pr en donde se encuentran los $\mathbb{Z}$-prerradicales de los que hablamos en los párrafos anteriores. 
Ejemplo 5.15. Retomemos el Ejemplo 5.13, donde $I=p^{2} \mathbb{Z}$ es un ideal de $\mathbb{Z}$, $R / I=\mathbb{Z} / p^{2} \mathbb{Z} \cong \mathbb{Z}_{p^{2}}$ y $\left\langle\mathbb{Z}\right.$-pr, $\varphi_{I}, \psi_{I}, \mathbb{Z}_{p^{2}}$-pr $\rangle$ es la conexión de Galois que corresponde en este caso. Entonces

1. $\operatorname{Soc}\left(\_\right) \in\left[\alpha_{\mathbb{Z}_{p}}^{\mathbb{Z}_{p}}, \omega_{\mathbb{Z}_{p}}^{\mathbb{Z}_{p^{2}}}\right]=\overleftarrow{\varphi_{I}}(1,0)$

2. $\operatorname{Rad}\left(\_\right) \in\left[\alpha_{\mathbb{Z}_{p}}^{\mathbb{Z}_{p^{2}}}, \omega_{0}^{\mathbb{Z}_{p}}\right]=\overleftarrow{\varphi_{I}}(0,1)$.

3. El radical idempotente $T\left(\_\right) \in\left[\alpha_{\mathbb{Z}_{p^{2}}}^{\mathbb{Z}_{p^{2}}}, 1_{\mathbb{Z}}\right]=\overleftarrow{\varphi_{I}}(1,1)$.

4. Los átomos de $\mathbb{Z}-p r,\left\{\alpha_{\mathbb{Z}_{q}}^{\mathbb{Z}_{q}} \mid\right.$ q es primo $\}$, se encuentran en $\left[0_{\mathbb{Z}}, \omega_{\mathbb{Z}_{p^{2}}}\right]=$ $\overleftarrow{\varphi_{I}}(0,0)$

5. Los coátomos de $\mathbb{Z}-p r,\left\{\omega_{q \mathbb{Z}}^{\mathbb{Z}} \mid\right.$ q es primo $\}$, se encuentran en $\left[\alpha_{\mathbb{Z}_{p^{2}}}^{\mathbb{Z}_{p^{2}}}, 1_{\mathbb{Z}}\right]=$ $\overleftarrow{\varphi_{I}}(1,1)$

\subsubsection{Prerradicales idempotentes $y$ radicales en $\mathbb{Z}$-pr}

En la sección anterior, de acuerdo a la partición de $\mathbb{Z}$-pr inducida por el ideal $I=p^{n} \mathbb{Z}$, se hallaron los intervalos en donde se encuentran ciertos prerradicales idempotentes y radicales. Lo anterior fué con base en el estudio de las clases de equivalencia de prerradicales en $\mathbb{Z}$-pr con respecto a la clase $\mathcal{C}_{I}$, es decir, la de los $\mathbb{Z} / p^{n} \mathbb{Z}$-módulos, o bien, al estudiar las imágenes inversas bajo $\varphi_{I}$, de los prerradicales idempotentes y radicales, de la conexión de Galois $\left\langle\varphi_{I}, \psi_{I}\right\rangle$, inducida por el ideal $I=p^{n} \mathbb{Z}$, donde el primo $p$ y $n$ estan fijos. En esta sección localizaremos a los prerradicales idempotentes y radicales en $\mathbb{Z}$-pr, variando los primos $p$ y las potencias $n$.

De la Proposición 4.28 se tiene que la partición $\mathcal{P}_{J}$ de $\mathbb{Z}$-pr es más fina que la partición $\mathcal{P}_{I}$ para los ideales $I=p^{n} \mathbb{Z}$ y $J=p^{n+1} \mathbb{Z}$ de $\mathbb{Z}$ con $p$ primo y $n \geq 1$, puesto que $J \subset I$. Este hecho lo usaremos más adelante.

Primero daremos el siguiente lema que se usará en muchos de los resultados siguientes.

Lema 5.16. Sean $\tau_{i}, \sigma_{i} \in R$-pr. Entonces $\bigcap_{i \in X}\left[\tau_{i}, \sigma_{i}\right]=\left[\bigvee_{i \in X} \tau_{i}, \bigwedge_{i \in X} \sigma_{i}\right]$.

Demostración. El resultado se tiene de la definición de $\bigvee_{i \in X} \tau_{i}$ y $\bigwedge_{i \in X} \sigma_{i}$.

Como ya mencionamos en esta sección estamos interesados en hallar de cierta forma zonas en la retícula $\mathbb{Z}$-pr en donde están los prerradicales idempotentes y radicales.

Primero notemos que al considerar al ideal $I=p^{n} \mathbb{Z}$ de $\mathbb{Z}$, por el Teorema 4.12 y de acuerdo a la partición de $\mathbb{Z}$-pr inducida por el ideal $I$, los prerradicales idempotentes en la retícula $\mathbb{Z}$-pr para cada primo $p$ y cada $n \geq 1$ se encuentran en:

$$
\mathcal{I}_{p}^{n}=\overleftarrow{\varphi_{I}}\left(0_{n}\right) \cup \overleftarrow{\varphi_{I}}(1,0, \cdots, 0) \cup \overleftarrow{\varphi_{I}}(1,1,0, \cdots, 0) \cup \cdots \cup \overleftarrow{\varphi_{I}}\left(1_{n}\right)
$$


Así que por el Teorema 5.7 y la Proposición 5.10, se tiene que

$$
\mathcal{I}_{p}^{n}=\left[0_{\mathbb{Z}}, \omega_{0}^{\mathbb{Z}_{p^{n}}}\right] \cup \bigcup_{m=1}^{n}\left[\alpha_{\mathbb{Z}_{p}^{m}}^{\mathbb{Z}_{p^{m}}}, \omega_{\mathbb{Z}_{p^{m}}}^{\mathbb{Z}_{p^{n}}}\right] .
$$

Es importante recalcar que en lo anterior el primo $p$ y $n$ están fijos. Si ahora vamos variando los ideales, es decir, consideramos los ideales $J=p^{n+1} \mathbb{Z}$ de $\mathbb{Z}$, donde el primo $p$ está fijo y vamos aumentando las potencias de $p$, entonces por la Proposición 4.28, la partición $\mathcal{P}_{J}$ de $\mathbb{Z}$-pr es más fina que la partición $\mathcal{P}_{I}$. De este modo, los funtores que inducen la conexión de Galois entre $\mathbb{Z}$-pr y $\mathbb{Z}_{p^{n} \text {-pr }}$ van cambiando dependiendo del ideal que estemos considerando y por tanto $\varphi_{I}$ también varía. Usemos la siguiente notación, para el ideal $I=p^{n} \mathbb{Z}$ y $\sigma \in \mathbb{Z}_{p^{n}}$-pr

$$
\overleftarrow{\varphi_{I}}(\sigma)=\overleftarrow{\varphi_{n}}(\sigma)
$$

Sea $\mathbb{P}=\{p \mid p$ primo $\}$. Para cada $p \in \mathbb{P}$ y al variar $n$, por (74) el siguiente diagrama ilustra a $\operatorname{los} \mathcal{I}_{p}^{n}$.

$$
\begin{array}{lll}
\mathcal{I}_{p}^{1} & =\overleftarrow{\varphi_{1}}(0) & \cup \overleftarrow{\varphi_{1}}(1) \\
\mathcal{I}_{p}^{2} & =\overleftarrow{\varphi_{2}}(0,0) \quad \cup \overleftarrow{\varphi_{2}}(1,0) \quad \cup \overleftarrow{\varphi_{2}}(1,1) \\
\mathcal{I}_{p}^{3} & =\overleftarrow{\varphi_{3}}(0,0,0) \quad \cup \overleftarrow{\varphi_{3}}(1,0,0) \quad \cup \overleftarrow{\varphi_{3}}(1,1,0) \quad \cup \overleftarrow{\varphi_{3}}(1,1,1)
\end{array}
$$

Del diagrama anterior, podemor decir que al variar $n, \operatorname{los} \mathcal{I}_{p}^{n}$ forman una matriz triangular superior infinita.

Lema 5.17. Sean $p$ un número primo y $n \geq 1$. Entonces $\mathcal{I}_{p}^{n+1} \subseteq \mathcal{I}_{p}^{n}$.

Demostración. Por (75), tenemos lo siguiente:

$$
\mathcal{I}_{p}^{n}=\left[0_{\mathbb{Z}}, \omega_{0}^{\mathbb{Z}_{p^{n}}}\right] \cup\left[\alpha_{\mathbb{Z}_{p}}^{\mathbb{Z}_{p}}, \omega_{\mathbb{Z}_{p}}^{\mathbb{Z}_{p^{n}}}\right] \cup\left[\alpha_{\mathbb{Z}_{p^{2}}}^{\mathbb{Z}_{p^{2}}}, \omega_{\mathbb{Z}_{p^{2}}}^{\mathbb{Z}_{p^{n}}}\right] \cup \cdots \cup\left[\alpha_{\mathbb{Z}_{p^{n}}}^{\mathbb{Z}_{p^{n}}}, \omega_{\mathbb{Z}_{p^{n}}}^{\mathbb{Z}_{p^{n}}}\right]
$$

y

$$
\begin{aligned}
\mathcal{I}_{p}^{n+1} & =\left[0_{\mathbb{Z}}, \omega_{0}^{\mathbb{Z}_{p^{n+1}}}\right] \cup\left[\alpha_{\mathbb{Z}_{p}}^{\mathbb{Z}_{p}}, \omega_{\mathbb{Z}_{p}}^{\mathbb{Z}_{p^{n+1}}}\right] \cup\left[\alpha_{\mathbb{Z}_{p^{2}}}^{\mathbb{Z}_{p^{2}}}, \omega_{\mathbb{Z}_{p^{2}}}^{\mathbb{Z}_{p^{n+1}}}\right] \cup \cdots \\
& \cup\left[\alpha_{\mathbb{Z}_{p^{n}}}^{\mathbb{Z}_{p^{n}}}, \omega_{\mathbb{Z}_{p^{n}}}^{\mathbb{Z}_{p^{n+1}}}\right] \cup\left[\alpha_{\mathbb{Z}_{p^{n+1}}}^{\mathbb{Z}^{n+1}}, \omega_{\mathbb{Z}_{p^{n+1}}}^{\mathbb{Z}_{n+1}}\right] .
\end{aligned}
$$

Por 4 del Corolario 5.6, se tiene que $\left[0_{\mathbb{Z}}, \omega_{0}^{\mathbb{Z}_{p^{n+1}}}\right] \subset\left[0_{\mathbb{Z}}, \omega_{0}^{\mathbb{Z}_{p^{n}}}\right]$. Por el Corolario 5.6, parte $5, \omega_{\mathbb{Z}_{p^{i}}}^{\mathbb{Z}_{p^{n+1}}} \prec \omega_{\mathbb{Z}_{p^{i}}}^{\mathbb{Z}_{p^{n}}}$ y así $\left[\alpha_{\mathbb{Z}_{p^{i}}}^{\mathbb{Z}_{p^{i}}}, \omega_{\mathbb{Z}_{p^{i}}}^{\mathbb{Z}_{p^{n+1}}}\right] \subset\left[\alpha_{\mathbb{Z}_{p^{i}}}^{\mathbb{Z}_{p^{i}}}, \omega_{\mathbb{Z}_{p^{i}}}^{\mathbb{Z}_{p^{n}}}\right]$. Se tiene que $\omega_{\mathbb{Z}_{p^{n}}}^{\mathbb{Z}_{p^{n}}}=1_{\mathbb{Z}}=\omega_{\mathbb{Z}_{p^{n+1}}}^{\mathbb{Z}_{p^{n+1}}}$ y por 1 del Corolario 5.6, $\alpha_{\mathbb{Z}_{p^{n}}}^{\mathbb{Z}_{p^{n}}} \prec \alpha_{\mathbb{Z}_{p^{n+1}}}$, de

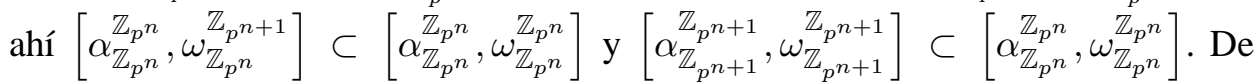
donde $\mathcal{I}_{p}^{n+1} \subseteq \mathcal{I}_{p}^{n}$. 
Proposición 5.18. Sea p un número primo. Entonces

$$
\bigcap_{n=1}^{\infty} \mathcal{I}_{p}^{n}=\left[0, \bigwedge_{n=1}^{\infty} \omega_{0}^{\mathbb{Z}_{p^{n}}}\right] \cup \bigcup_{m=1}^{\infty}\left[\alpha_{\mathbb{Z}_{p^{m}}}^{\mathbb{Z}_{p^{m}}}, \bigwedge_{n=m+1}^{\infty} \omega_{\mathbb{Z}_{p^{m}}}^{\mathbb{Z}_{p^{n}}}\right] \cup\left[\bigvee_{n=1}^{\infty} \alpha_{\mathbb{Z}_{p^{n}}}^{\mathbb{Z}_{p^{n}}}, 1\right]
$$

Demostración. Sea $\tau \in \bigcap_{n=1}^{\infty} \mathcal{I}_{p}^{n}$. Entonces $\tau \in \mathcal{I}_{p}^{n}$, para toda $n \geq 1$. Es decir, $\tau$ está en todos los renglones de la matriz infinita (76) y se tiene los siguientes casos: Caso 1. Si $\tau \in \overleftarrow{\varphi_{n}}\left(0_{n}\right)=\left[0_{\mathbb{Z}}, \omega_{0}^{\mathbb{Z}_{p^{n}}}\right]$ para toda $n \geq 1$, es decir, $\tau$ está en la primera columna de la matriz (76), entonces por el Lema 5.16 se tiene que $\tau \in$ $\left[0_{\mathbb{Z}}, \bigwedge_{n=1}^{\infty} \omega_{0}^{\mathbb{Z}_{p^{n}}}\right]$.

Caso 2. Si $\tau \in \overleftarrow{\varphi_{n}}\left(1_{n}\right)=\left[\alpha_{\mathbb{Z}_{p^{n}}}^{\mathbb{Z}_{p^{n}}}, 1_{\mathbb{Z}}\right]$ para toda $n \geq 1$, es decir, $\tau$ está en la diagonal de la matriz (76), entonces por el Lema 5.16 tenemos que $\tau \in\left[\bigvee_{n=1}^{\infty} \alpha_{\mathbb{Z}_{p^{n}}}^{\mathbb{Z}_{p^{n}}}, 1\right]$.

Caso 3. O bien, $\tau \notin \overleftarrow{\varphi_{n_{1}}}\left(0_{n_{1}}\right)$ para algún $n_{1}$ tal que $1 \leq n_{1}$ y $\tau \notin \overleftarrow{\varphi_{n_{2}}}\left(1_{n_{2}}\right)$ para algún $n_{2}$ tal que $1 \leq n_{2}$. Entonces por el Lema 5.17, $\tau \notin \overleftarrow{\varphi_{j}}\left(0_{j}\right)$ para toda $j>$ $n_{1}$ y $\tau \notin \overleftarrow{\varphi_{k}}\left(1_{k}\right)$ para toda $k>n_{2}$. También, para toda $k>n_{2}, \tau \notin \overleftarrow{\varphi_{k}}(\sigma)$, donde $\sigma$ es un $\mathbb{Z}_{p^{k}}$-prerradical idempotente con $n_{2}$ la longitud del segmento inicial de entradas el número 1 de la sucesión binaria que representa a $\sigma$. Sea $l=\max \left\{n_{1}, n_{2}\right\}$. Entonces por el Lema 5.16 y el Lema 5.17, $\tau \in \bigcup_{m=1}^{l-1}\left[\begin{array}{c}\mathbb{Z}_{p^{m}} \\ \mathbb{Z}_{p^{m}}\end{array} \bigwedge_{i=m+1}^{l} \omega_{\mathbb{Z}_{p^{m}}}^{\mathbb{Z}_{p^{i}}}\right]$.

De los tres casos anteriores se concluye que

$$
\bigcap_{n=1}^{\infty} \mathcal{I}_{p}^{n} \subseteq\left[0, \bigwedge_{n=1}^{\infty} \omega_{0}^{\mathbb{Z}_{p^{n}}}\right] \cup \bigcup_{m=1}^{\infty}\left[\alpha_{\mathbb{Z}_{p^{m}}}^{\mathbb{Z}_{p^{m}}}, \bigwedge_{n=m+1}^{\infty} \omega_{\mathbb{Z}_{p^{n}}}^{\mathbb{Z}_{p^{n}}}\right] \cup\left[\bigvee_{n=1}^{\infty} \alpha_{\mathbb{Z}_{p^{n}}}^{\mathbb{Z}_{p^{n}}}, 1\right]
$$

La otra contención es fácil de verificar.

Las particiones de la retícula $\mathbb{Z}$-pr que hemos estudiado en este trabajo dependen de los ideales $I=p^{n} \mathbb{Z}$ y por lo anterior ya sabemos cuales son las zonas de prerradicales idempotentes en $\mathbb{Z}$-pr cuando consideramos un ideal con $p$ fijo y variamos $n$. Por lo que podemos concluir que los prerradicales idempotentes en $\mathbb{Z}$-pr variando ahora los primos se encuentran en:

$$
\begin{aligned}
\mathbb{Z} \text {-idem } & \subset \bigcap_{p \in \mathbb{P}} \bigcap_{n=1}^{\infty} \mathcal{I}_{p}^{n} \\
& =\bigcap_{p \in \mathbb{P}}\left(\left[0, \bigwedge_{n=1}^{\infty} \omega_{0}^{\mathbb{Z}_{p^{n}}}\right] \cup \bigcup_{m=1}^{\infty}\left[\alpha_{\mathbb{Z}_{p}^{m}}^{\mathbb{Z}_{p^{m}}}, \bigwedge_{n=m+1}^{\infty} \omega_{\mathbb{Z}_{p} m}^{\mathbb{Z}_{p^{n}}}\right] \cup\left[\bigvee_{n=1}^{\infty} \alpha_{\mathbb{Z}_{p}}^{\mathbb{Z}_{p^{n}}}, 1\right]\right)
\end{aligned}
$$


Usando (77) podemos hallar en la retícula $\mathbb{Z}$-pr al perradical idempotente Soc(_). En la sección precedente ya habíamos visto este ejemplo considerando un primo $p$ y $n \geq 1$ fijos.

Ejemplo 5.19. En $\mathbb{Z}$-pr se tiene que Soc $\left(_{-}\right)=\bigvee_{q \text { primo }} \alpha_{\mathbb{Z}_{q}}^{\mathbb{Z}_{q}}$ y del Ejemplo 1.70 sabemos que Soc(_) es un prerradical idempotente. Considerando el ideal $I=p^{n} \mathbb{Z}$ de $\mathbb{Z}$, por (68), tenemos que para tal primo p y $n \geq 1$ fijos:

$$
\operatorname{Soc}\left(\_\right) \in\left[\alpha_{\mathbb{Z}_{p}}^{\mathbb{Z}_{p}}, \omega_{\mathbb{Z}_{p}}^{\mathbb{Z}_{p^{n}}}\right]=\overleftarrow{\varphi_{I}}(1,0,0, \cdots, 0)
$$

Es decir, para un primo fijo p y variando n, el prerradical Soc(_) siempre está en la segunda columna (de izquierda a derecha) de la matriz triangular superior infinita (76). Así por el Lema 5.16:

$$
\operatorname{Soc}\left(\_\right) \in \bigcap_{n=2}^{\infty}\left[\alpha_{\mathbb{Z}_{p}}^{\mathbb{Z}_{p}}, \omega_{\mathbb{Z}_{p}}^{\mathbb{Z}_{p^{n}}}\right]=\left[\alpha_{\mathbb{Z}_{p}}^{\mathbb{Z}_{p}}, \bigwedge_{n=2}^{\infty} \omega_{\mathbb{Z}_{p}}^{\mathbb{Z}_{p^{n}}}\right]
$$

Lo anterior dice en la Proposición 5.18 que Soc $\left(\_\right) \in \bigcup_{m=1}^{\infty}\left[\alpha_{\mathbb{Z}_{p} m}^{\mathbb{Z}_{p^{m}}}, \bigwedge_{n=m+1}^{\infty} \omega_{\mathbb{Z}_{p} m}^{\mathbb{Z}_{p^{n}}}\right]$ considerando solamente $m=1$, pues recuerde que $m$ es la longitud del segmento inicial con entradas el número 1 de la sucesión binaria que representa a los $\mathbb{Z}_{p^{n} \text { - }}$ prerradicales idempotentes. Así por (77) y de nuevo el Lema 5.16 obtenemos:

$$
\operatorname{Soc}\left(\_\right) \in \bigcap_{p \in \mathbb{P}}\left[\alpha_{\mathbb{Z}_{p}}^{\mathbb{Z}_{p}}, \bigwedge_{n=2}^{\infty} \omega_{\mathbb{Z}_{p}}^{\mathbb{Z}_{p^{n}}}\right]=\left[\bigvee_{p \in \mathbb{P}} \alpha_{\mathbb{Z}_{p}}^{\mathbb{Z}_{p}}, \bigwedge_{p \in \mathbb{P}} \bigwedge_{n=2}^{\infty} \omega_{\mathbb{Z}_{p}}^{\mathbb{Z}_{p^{n}}}\right]
$$

Los átomos aunque no son prerradicales idempontentes los podemos localizar en la retícula $\mathbb{Z}$-pr usando (76).

Ejemplo 5.20. Considerando el ideal $I=p^{n} \mathbb{Z}$ de $\mathbb{Z}$, por (72) tenemos que los atomos de $\mathbb{Z}$-pr se encuentran en:

$$
\left\{\alpha_{\mathbb{Z}_{q}}^{\mathbb{Z}_{q} \infty} \mid \text { qes primo }\right\} \subset\left[0_{\mathbb{Z}}, \omega_{0}^{\mathbb{Z}_{p}{ }^{n}}\right]=\overleftarrow{\varphi_{I}}\left(0_{n}\right)
$$

para cada p primo y cada $n \geq 1$. Entonces para cada $p \in \mathbb{P}$ y variando $n$, los átomos están en la primera columna (de izquierda a derecha) de (76) y por el Lema 5.16:

con $q \in \mathbb{P}$.

$$
\alpha_{\mathbb{Z}_{q}}^{\mathbb{Z}_{q} \infty} \in \bigcap_{n=1}^{\infty}\left[0_{\mathbb{Z}}, \omega_{0}^{\mathbb{Z}_{p^{n}}}\right]=\left[0_{\mathbb{Z}}, \bigwedge_{n=1}^{\infty} \omega_{0}^{\mathbb{Z}_{p^{n}}}\right]
$$

Por lo tanto variando los primos obtenemos que los átomos de $\mathbb{Z}$-pr se encuentran en:

$$
\alpha_{\mathbb{Z}_{q}}^{\mathbb{Z}_{q^{\infty}}} \in \bigcap_{p \in \mathbb{P}}\left[0_{\mathbb{Z}}, \bigwedge_{n=1}^{\infty} \omega_{0}^{\mathbb{Z}_{p^{n}}}\right]=\left[0_{\mathbb{Z}}, \bigwedge_{p \in \mathbb{P} n=1}^{\infty} \bigwedge_{n=1}^{\mathbb{Z}_{p^{n}}}\right]
$$

con $q \in \mathbb{P}$. 
Análogamente al procedimiento anterior encontramos las regiones en la retícula $\mathbb{Z}$-pr en donde se encuentran los radicales de acuerdo a la partición inducida por el ideal $I=p^{n} \mathbb{Z}$. Así por el Teorema 4.13 los $\mathbb{Z}$-radicales para cada $p \in \mathbb{P}$ y cada $n \geq 1$, se encuentran en:

$$
\mathcal{R}_{p}^{n}=\overleftarrow{\varphi_{I}}\left(0_{n}\right) \cup \overleftarrow{\varphi_{I}}(0,1, \cdots, 1) \cup \overleftarrow{\varphi_{I}}(0,0,1, \cdots, 1) \cup \cdots \cup \overleftarrow{\varphi_{I}}\left(1_{n}\right)
$$

y por el Teorema 5.9, la Proposición 5.10 y el Corolario 5.8, se tiene que

$$
\mathcal{R}_{p}^{n}=\left[0, \omega_{0}^{\mathbb{Z}_{p^{n}}}\right] \cup \bigcup_{k=1}^{n-1}\left[\alpha_{\mathbb{Z}_{p^{n-k}}}^{\mathbb{Z}_{p^{n}}}, \omega_{0}^{\mathbb{Z}_{p^{k}}}\right] \cup\left[\alpha_{\mathbb{Z}_{p^{n}}}^{\mathbb{Z}_{p^{n}}}, 1\right]
$$

Para cada $p \in \mathbb{P}$ y al variar $n$, por (78), el siguiente diagrama ilustra a los $\mathcal{R}_{p}^{n}$.

$$
\begin{aligned}
& \mathcal{R}_{p}^{1}=\quad \overleftarrow{\varphi_{1}}(0) \quad \cup \overleftarrow{\varphi_{1}}(1) \\
& \mathcal{R}_{p}^{2}=\quad \overleftarrow{\varphi_{2}}(0,0) \quad \cup \overleftarrow{\varphi_{2}}(0,1) \quad \cup \overleftarrow{\varphi_{2}}(1,1) \\
& \mathcal{R}_{p}^{3}=\overleftarrow{\varphi_{3}}(0,0,0) \cup \overleftarrow{\varphi_{3}}(0,0,1) \cup \overleftarrow{\varphi_{3}}(0,1,1) \cup \overleftarrow{\varphi_{3}}(1,1,1)
\end{aligned}
$$

Al variar $n$ podemos pensar que los $\mathcal{R}_{p}^{n}$ forman una matriz triangular superior infinita.

Lema 5.21. Sean $p$ un número primo y $n \geq 1$. Entonces $\mathcal{R}_{p}^{n+1} \subseteq \mathcal{R}_{p}^{n}$.

Demostración. La prueba es dual a la del Lema 5.17.

Proposición 5.22. Sea p un número primo. Entonces

$$
\bigcap_{n=1}^{\infty} \mathcal{R}_{p}^{n}=\left[0, \bigwedge_{n=1}^{\infty} \omega_{0}^{\mathbb{Z}_{p^{n}}}\right] \cup \bigcup_{k=1}^{\infty}\left[\bigvee_{n=k+1}^{\infty} \alpha_{\mathbb{Z}_{p^{n-k}}}^{\mathbb{Z}_{p^{n}}}, \omega_{0}^{\mathbb{Z}_{p^{k}}}\right] \cup\left[\bigvee_{n=1}^{\infty} \alpha_{\mathbb{Z}_{p^{n}}}^{\mathbb{Z}_{p^{n}}}, 1\right] .
$$

Demostración. La prueba es similar a la de la Proposición 5.18.

Concluimos entonces que los radicales en $\mathbb{Z}$-pr variando los primos se encuentran en:

$$
\begin{aligned}
\mathbb{Z}-\operatorname{rad} & \subset \bigcap_{p \in \mathbb{P}} \bigcap_{n=1}^{\infty} \mathcal{R}_{p}^{n} \\
& =\bigcap_{p \in \mathbb{P}}\left(\left[0, \bigwedge_{n=1}^{\infty} \omega_{0}^{\mathbb{Z}_{p^{n}}}\right] \cup \bigcup_{k=1}^{\infty}\left[\bigvee_{n=k+1}^{\infty} \alpha_{\mathbb{Z}_{p^{n-k}}}^{\mathbb{Z}_{p^{n}}}, \omega_{0}^{\mathbb{Z}_{p^{k}}}\right] \cup\left[\bigvee_{n=1}^{\infty} \alpha_{\mathbb{Z}_{p^{n}}}^{\mathbb{Z}_{p^{n}}}, 1\right]\right) .
\end{aligned}
$$

El siguiente ejemplo muestra el intervalo en $\mathbb{Z}$-pr en donde se encuentra el radical $\operatorname{Rad}\left(\_\right)$, el cual ya habíamos visto en la sección anterior considerando un primo $p$ y $n \geq 1$ fijos. 
Ejemplo 5.23. En $\mathbb{Z}$-pr se tiene que $\operatorname{Rad}\left(\_\right)=\bigwedge_{q \text { primo }} \omega_{0}^{\mathbb{Z}_{q}}$ y del Ejemplo 1.70 sabemos que Rad(_) es un radical. Considerando el ideal $I=p^{n} \mathbb{Z}$ de $\mathbb{Z}$, por (70), tenemos que para tal primo $p$ y $n \geq 1$ fijos:

$$
\operatorname{Rad}\left(\_\right) \in\left[\alpha_{\mathbb{Z}_{p^{n-1}}}^{\mathbb{Z}_{p^{n}}}, \omega_{0}^{\mathbb{Z}_{p}}\right]=\overleftarrow{\varphi_{I}}(0,1,1, \cdots, 1)
$$

Es decir, para un primo fijo p y variando n, el prerradical Rad(_) siempre está en la segunda columna (de derecha a izquierda) de la matriz triangular superior infnita (80). Así por el Lema 5.16:

$$
\operatorname{Rad}\left(\_\right) \in \bigcap_{n=2}^{\infty}\left[\alpha_{\mathbb{Z}_{p^{n-1}}}^{\mathbb{Z}_{p^{n}}}, \omega_{0}^{\mathbb{Z}_{p}}\right]=\left[\bigvee_{n=2}^{\infty} \alpha_{\mathbb{Z}_{p^{n-1}}}^{\mathbb{Z}_{p^{n}}}, \omega_{0}^{\mathbb{Z}_{p}}\right]
$$

Lo anterior dice en la Proposición 5.22 que $\operatorname{Rad}\left(\_\right) \in \bigcup_{k=1}^{\infty}\left[\bigvee_{n=k+1}^{\infty} \alpha_{\mathbb{Z}_{p^{n-k}}}^{\mathbb{Z}_{p^{n}}}, \omega_{0}^{\mathbb{Z}_{p^{k}}}\right]$ considerando solamente $k=1$, pues recuerde que $k$ es la longitud del segmento inicial con entradas el número 0 de la sucesión binaria que representa a los $\mathbb{Z}_{p^{n} \text { - }}$ radicales. Así por (81) y de nuevo el Lema 5.16 obtenemos:

$$
\operatorname{Rad}\left(\_\right) \in \bigcap_{p \in \mathbb{P}}\left[\bigvee_{n=2}^{\infty} \alpha_{\mathbb{Z}_{p^{n-1}}}^{\mathbb{Z}_{p^{n}}}, \omega_{0}^{\mathbb{Z}_{p}}\right]=\left[\bigvee_{p \in \mathbb{P}} \bigvee_{n=2}^{\infty} \alpha_{\mathbb{Z}_{p^{n-1}}}^{\mathbb{Z}_{p^{n}}}, \bigwedge_{p \in \mathbb{P}} \omega_{0}^{\mathbb{Z}_{p}}\right]
$$

Los coátomos aunque no son radicales los podemos localizar en la retícula $\mathbb{Z}$-pr usando (80).

Ejemplo 5.24. Considerando el ideal $I=p^{n} \mathbb{Z}$ de $\mathbb{Z}$, por (73) tenemos que los coátomos de $\mathbb{Z}$-pr se encuentran en:

$$
\left\{\omega_{q \mathbb{Z}}^{\mathbb{Z}} \mid \text { q es primo }\right\} \subset\left[\alpha_{\mathbb{Z}_{p^{n}}}^{\mathbb{Z}_{p^{n}}}, 1_{\mathbb{Z}}\right]=\overleftarrow{\varphi}_{I}\left(1_{n}\right)
$$

para cada p primo y cada $n \geq 1$. Entonces para cada $p \in \mathbb{P}$ y variando $n$, los coátomos están en la primera columna (de derecha a izquierda) de (80) y por el Lema 5.16:

$$
\omega_{q \mathbb{Z}}^{\mathbb{Z}} \in \bigcap_{n=1}^{\infty}\left[\alpha_{\mathbb{Z}_{p^{n}}}^{\mathbb{Z}_{p^{n}}}, 1_{\mathbb{Z}}\right]=\left[\bigvee_{n=1}^{\infty} \alpha_{\mathbb{Z}_{p^{n}}}^{\mathbb{Z}_{p^{n}}}, 1_{\mathbb{Z}}\right]
$$

con $q \in \mathbb{P}$.

Por lo tanto variando los primos obtenemos que los coátomos de $\mathbb{Z}$-pr se encuentran en:

$$
\omega_{q \mathbb{Z}}^{\mathbb{Z}} \in \bigcap_{p \in \mathbb{P}}\left[\bigvee_{n=1}^{\infty} \alpha_{\mathbb{Z}_{p^{n}}}^{\mathbb{Z}_{p^{n}}}, 1_{\mathbb{Z}}\right]=\left[\bigvee_{p \in \mathbb{P}} \bigvee_{n=1}^{\infty} \alpha_{\mathbb{Z}_{p^{n}}}^{\mathbb{Z}_{p^{n}}}, 1_{\mathbb{Z}}\right]
$$

con $q \in \mathbb{P}$. 
Observación 5.25. Notemos que para realizar el Ejemplo 5.20 se puede usar (80) en lugar de (76). Pero es mejor usar (76), puesto que ahí se aprecian las columnas de $\overleftarrow{\varphi_{I}}\left(0_{n}\right)$ para cada $n \geq 1$. Dualmente, en el Ejemplo 5.24 también se puede usar (76) en lugar de (80).

\subsubsection{Radicales idempotentes en $\mathbb{Z}$-pr}

En esta sección siguiendo procedimientos análogos a los de la sección anterior encontraremos las regiones en la retícula $\mathbb{Z}$-pr en donde se encuentran los radicales idempotentes, a diferencia de los prerradicales idempotentes y radicales, la descripción de los intervalos en $\mathbb{Z}$-pr para dichos prerradicales quedan descritos de una manera más sencilla como veremos en lo que sigue.

Empezamos esta sección con el siguiente lema.

Lema 5.26. Sean $p, q$ números primos y sean $n, m \geq 1$. Entonces:

1. Si $p \neq q$, entonces $\alpha_{\mathbb{Z}_{p^{n}}}^{\mathbb{Z}_{p^{n}}} \preceq \omega_{0}^{\mathbb{Z}_{q^{m}}}$.

2. Si $p=q$, entonces $\alpha_{\mathbb{Z}_{p^{n}}}^{\mathbb{Z}_{p^{n}}} \npreceq \omega_{0}^{\mathbb{Z}_{q^{m}}}$.

Demostración. Si $p \neq q$, entonces $\alpha_{\mathbb{Z}_{p^{n}}}^{\mathbb{Z}_{p^{n}}}\left(\mathbb{Z}_{q^{m}}\right)=0$, por lo que $\alpha_{\mathbb{Z}_{p^{n}}}^{\mathbb{Z}_{p^{n}}} \preceq \omega_{0}^{\mathbb{Z}_{q^{m}}}$, puesto que por la Observación 1.57, $\omega_{0}^{\mathbb{Z}_{q} m}$ es el mayor prerradical que manda $\mathbb{Z}_{q^{m}}$ a 0 . Por otro lado, para $n, m \geq 1$, por el Lema 5.5, se tiene que $\alpha_{\mathbb{Z}_{p^{n}}}^{\mathbb{Z}_{p^{n}}}\left(\mathbb{Z}_{p^{m}}\right) \neq 0$, así que $\alpha_{\mathbb{Z}_{p^{n}}}^{\mathbb{Z}_{p^{n}}} \npreceq \omega_{0}^{\mathbb{Z}_{p^{m}}}$.

Para el ideal $I=p^{n} \mathbb{Z}$, por las Proposiciones 4.12 y 4.13 para cada $p \in \mathbb{P}$ y cada $n \geq 1$, se tiene que los radicales idempotentes en $\mathbb{Z}$-pr se encuentran en:

$$
\mathcal{D}_{p}^{n}=\overleftarrow{\varphi_{I}}\left(0_{n}\right) \cup \overleftarrow{\varphi_{I}}\left(1_{n}\right)
$$

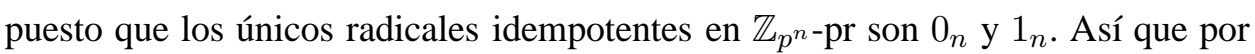
la Proposición 5.10 y el Corolario 5.8:

$$
\mathcal{D}_{p}^{n}=\left[0_{\mathbb{Z}}, \omega_{0}^{\mathbb{Z}_{p^{n}}}\right] \cup\left[\alpha_{\mathbb{Z}_{p^{n}}}^{\mathbb{Z}_{p^{n}}}, 1_{\mathbb{Z}}\right]
$$

Por el Lema 5.26, para cada $p \in \mathbb{P}$ y cada $n \geq 1$, la unión de los intervalos que aparece en (83) es disjunta.

Lema 5.27. Sean p un número primo y $n \geq 1$. Entonces $\mathcal{D}_{p}^{n+1} \subseteq \mathcal{D}_{p}^{n}$.

Demostración. El resultado se tiene de 1 y 4 del Corolario 5.6.

Proposición 5.28. Sea p un número primo. Entonces

$$
\bigcap_{n=1}^{\infty} \mathcal{D}_{p}^{n}=\left[0_{\mathbb{Z}}, \bigwedge_{n=1}^{\infty} \omega_{0}^{\mathbb{Z}_{p^{n}}}\right] \cup\left[\bigvee_{n=1}^{\infty} \alpha_{\mathbb{Z}_{p^{n}}}^{\mathbb{Z}_{n}}, 1_{\mathbb{Z}}\right] .
$$


Demostración. Por el Lema 5.16 y el Lema 5.27:

$$
\begin{aligned}
\bigcap_{n=1}^{\infty} \mathcal{D}_{p}^{n} & =\left(\bigcap_{n=1}^{\infty}\left[0_{\mathbb{Z}}, \omega_{0}^{\mathbb{Z}_{p^{n}}}\right]\right) \cup\left(\bigcap_{n=1}^{\infty}\left[\alpha_{\mathbb{Z}_{p^{n}}}^{\mathbb{Z}_{n}}, 1_{\mathbb{Z}}\right]\right) \\
& =\left[0_{\mathbb{Z}}, \bigwedge_{n=1}^{\infty} \omega_{0}^{\mathbb{Z}_{p^{n}}}\right] \cup\left[\bigvee_{n=1}^{\infty} \alpha_{\mathbb{Z}_{p^{n}}}^{\mathbb{Z}_{p^{n}}}, 1_{\mathbb{Z}}\right]
\end{aligned}
$$

Denotaremos a la clase de los radicales idempotentes sobre un anillo $R$ como $R$-radidem. Concluimos entonces que al variar las potencias $n$ y los primos $p$ en los ideales $I=p^{n} \mathbb{Z}$, los prerradicales radicales idempotentes en $\mathbb{Z}$-pr se encuentran en

$$
\begin{aligned}
\mathbb{Z} \text {-radidem } & \subset \bigcap_{p \in \mathbb{P}} \bigcap_{n=1}^{\infty} \mathcal{D}_{p}^{n} \\
& =\bigcap_{p \in \mathbb{P}}\left(\left[0_{\mathbb{Z}}, \bigwedge_{n=1}^{\infty} \omega_{0}^{\mathbb{Z}_{p^{n}}}\right] \bigcup\left[\bigvee_{n=1}^{\infty} \alpha_{\mathbb{Z}_{p^{n}}}^{\mathbb{Z}_{p}}, 1_{\mathbb{Z}}\right]\right) .
\end{aligned}
$$

Notamos que la descripción de los intervalos en donde estan los $\mathbb{Z}$-radicales idempotentes, son en cierta forma accesibles para obtener más información de ellos. Por simplicidad, para cada $p \in \mathbb{P}$, usaremos la siguiente notación:

$$
\alpha_{p}=\bigvee_{n=1}^{\infty} \alpha_{\mathbb{Z}_{p^{n}}}^{\mathbb{Z}_{p^{n}}} \text { y } \omega_{p}=\bigwedge_{n=1}^{\infty} \omega_{0}^{\mathbb{Z}_{p^{n}}}
$$

Entonces reescribimos (84) como:

$$
\mathbb{Z} \text {-radidem } \subset \bigcap_{p \in \mathbb{P}}\left(\left[0_{\mathbb{Z}}, \omega_{p}\right] \cup\left[\alpha_{p}, 1_{\mathbb{Z}}\right]\right)
$$

El siguiente ejemplo muestra el intervalo en $\mathbb{Z}$-pr en donde se encuentra el radical idempotente $\mathrm{T}\left(\_\right)$, el cual ya habíamos visto en (71) considerando un primo $p$ y $n \geq 1$ fijos.

Ejemplo 5.29. Considerando el ideal $I=p^{n} \mathbb{Z}$ de $\mathbb{Z}$, en (71) obtuvimos que el radical idempotentente $T\left({ }_{(}\right)$sobre $\mathbb{Z}$ se encuentra en:

$$
T\left(\_\right) \in\left[\alpha_{\mathbb{Z}_{p^{n}}}^{\mathbb{Z}_{p^{n}}}, 1_{\mathbb{Z}}\right]=\overleftarrow{\varphi_{I}}\left(1_{n}\right)
$$

para cada $p \in \mathbb{P}$ y cada $n \geq 1$. Por la proposición 5.28 para cada $p \in \mathbb{P}$ :

$$
T\left(\_\right) \in\left[\bigvee_{n=1}^{\infty} \alpha_{\mathbb{Z}_{p^{n}}}^{\mathbb{Z}_{p^{n}}}, 1_{\mathbb{Z}}\right] \text {. }
$$


Así por (85) y de nuevo el Lema 5.16 obtenemos que en la reícula $\mathbb{Z}$-pr:

$$
T\left(\_\right) \in \bigcap_{p \in \mathbb{P}}\left[\alpha_{p}, 1_{\mathbb{Z}}\right]=\left[\bigvee_{p \in \mathbb{P}} \bigvee_{n=1}^{\infty} \alpha_{\mathbb{Z}_{p} p^{n}}^{\mathbb{Z}_{p^{n}}}, 1_{\mathbb{Z}}\right]
$$

Ahora mostraremos una descripción más sencilla de (85).

Diremos que $(A, B)$ es una bipartición de $\mathbb{P}$, si $A \cup B=\mathbb{P}$ y $A \cap B=$ $\emptyset$. Observe que $A$ o $B$ pueden ser el conjunto vacío. Denotaremos por $\mathcal{B}(\mathbb{P})$ al conjunto de todas las biparticiones de $\mathbb{P}$.

Proposición 5.30. $\bigcap_{p \in \mathbb{P}}\left(\left[0_{\mathbb{Z}}, \omega_{p}\right] \cup\left[\alpha_{p}, 1_{\mathbb{Z}}\right]\right)=\bigcup_{(A, B) \in \mathcal{B}(\mathbb{P})}\left[\bigvee_{p \in A} \alpha_{p}, \bigwedge_{q \in B} \omega_{q}\right]$.

Demostración. Sea $\sigma \in \bigcap_{p \in \mathbb{P}}\left(\left[0_{\mathbb{Z}}, \omega_{p}\right] \cup\left[\alpha_{p}, 1_{\mathbb{Z}}\right]\right)$. Definimos los siguientes conjuntos:

$$
\begin{aligned}
& A_{\sigma}=\left\{p \in \mathbb{P} \mid \sigma \in\left[\alpha_{p}, 1_{\mathbb{Z}}\right]\right\}, \\
& B_{\sigma}=\left\{q \in \mathbb{P} \mid \sigma \in\left[0_{\mathbb{Z}}, \omega_{q}\right]\right\} .
\end{aligned}
$$

Primero probemos que $\left(A_{\sigma}, B_{\sigma}\right)$ es una bipartición de $\mathbb{P}$. Si $p \in \mathbb{P}$, entonces $\sigma \in$ $\left[0_{\mathbb{Z}}, \omega_{p}\right]$ o $\sigma \in\left[\alpha_{p}, 1_{\mathbb{Z}}\right]$, es decir, $p \in B_{\sigma}$ o $p \in A_{\sigma}$. Por lo que $A_{\sigma} \cup B_{\sigma}=\mathbb{P}$. Por otro lado, si $p \in A_{\sigma}$ y $p \in B_{\sigma}$, entonces $\sigma \in\left[0_{\mathbb{Z}}, \omega_{p}\right] \cap\left[\alpha_{p}, 1_{\mathbb{Z}}\right]$, por lo que $\alpha_{p} \preceq \sigma \preceq \omega_{p}$. De ahí y por la definición de $\alpha_{p}$ y $\omega_{p}, \alpha_{\mathbb{Z}_{p^{n}}}^{\mathbb{Z}^{n}} \preceq \omega_{0}^{\mathbb{Z}_{p^{n}}}$, lo cual contradice el Lema 5.26 y entonces $A_{\sigma} \cap B_{\sigma}=\emptyset$. Por lo tanto concluimos que $\left(A_{\sigma}, B_{\sigma}\right)$ es una bipartición de $\mathbb{P}$.

Por definición de $A_{\sigma}$ y $B_{\sigma}$, tenemos

$$
\begin{aligned}
\sigma & \in\left(\bigcap_{q \in B_{\sigma}}\left[0_{\mathbb{Z}}, \omega_{q}\right]\right) \cap\left(\bigcap_{p \in A_{\sigma}}\left[\alpha_{p}, 1_{\mathbb{Z}}\right]\right) \\
& =\left[0_{\mathbb{Z}}, \bigwedge_{q \in B_{\sigma}} \omega_{q}\right] \cap\left[\bigvee_{p \in A_{\sigma}} \alpha_{p}, 1_{\mathbb{Z}}\right] \\
& =\left[\bigvee_{p \in A_{\sigma}} \alpha_{p}, \bigwedge_{q \in B_{\sigma}} \omega_{q}\right] .
\end{aligned}
$$

Donde la última igualdad se da por el Lema 5.26, pues para cada $p \in A_{\sigma}$ y $q \in B_{\sigma}$, teneos $\alpha_{\mathbb{Z}_{p^{n}}}^{\mathbb{Z}_{p^{n}}} \preceq \omega_{0}^{\mathbb{Z}_{q^{n}}}$, por lo que $\bigvee_{p \in A_{\sigma}} \alpha_{p} \preceq \bigwedge_{q \in B_{\sigma}} \omega_{q}$. 
Para la otra contención, sea $\sigma \in\left[\bigvee_{p \in A} \alpha_{p}, \bigwedge_{q \in B} \omega_{q}\right]$ para alguna bipartición $(A, B)$ de $\mathbb{P}$ y sea $p_{1} \in \mathbb{P}=A \cup B$. Si $p_{1} \in A$, entonces $\alpha_{p_{1}} \preceq \bigvee_{p \in A} \alpha_{p} \preceq \sigma$. Si $p_{1} \in B$, entonces $\sigma \preceq \bigwedge_{q \in B} \omega_{q} \preceq \omega_{p_{1}}$. Entonces $\sigma \in\left[0_{\mathbb{Z}}, \omega_{p_{1}}\right] \cup\left[\alpha_{p_{1}}, 1_{\mathbb{Z}}\right]$.

El siguiente corolario es consecuencia inmediata de la Proposición 5.30.

Corolario 5.31. $\mathbb{Z}$-radidem $\subset \bigcup_{(A, B) \in \mathcal{B}(\mathbb{P})}\left[\bigvee_{p \in A} \alpha_{p}, \bigwedge_{q \in B} \omega_{q}\right]$

Observación 5.32. Notemos que en la Proposición 5.30, podemos considerar el caso en el que solamente hay una bipartición de $\mathbb{P}$ y en donde A o B son el vacío. Es decir, si $A=\emptyset$, entonces $B=\mathbb{P}$ y en este caso $\mathbb{Z}$-radidem $\subset\left[0_{\mathbb{Z}}, \bigwedge_{q \in B} \omega_{q}\right]$, es decir, los prerradicales radicales idempotentes estan en el intervalo que corresponde $a \bigcap_{n=1}^{\infty} \overleftarrow{\varphi_{n}}\left(0_{n}\right)$. Análogamente si $B=\emptyset$

Corolario 5.33. Sea $p \in \mathbb{P}$ y $\sigma \in \mathbb{Z}$-radidem. Entonces, una y solo una, de las siguientes afirmaciones se cumple:

1. $\mathbb{Z}_{p^{n}} \in \mathbb{T}_{\sigma}$, para toda $n \geq 1$.

2. $\mathbb{Z}_{p^{n}} \in \mathbb{F}_{\sigma}$, parta toda $n \geq 1$.

Demostración. Por (85), si $\sigma \in \mathbb{Z}$-radidem, entonces para cada $p \in \mathbb{P}, \sigma \in$ $\left[0_{\mathbb{Z}}, \omega_{p}\right]$ o $\sigma \in\left[\alpha_{p}, 1_{\mathbb{Z}}\right]$, pero no en ambos, ya que tales intervalos son ajenos. Si $\sigma \in\left[0_{\mathbb{Z}}, \omega_{p}\right]$, entonces $\sigma \preceq \omega_{0}^{\mathbb{Z}_{p^{n}}}$ para toda $n \geq 1$ y por la Observación 1.57, $\sigma\left(\mathbb{Z}_{p^{n}}\right)=0$ para toda $n \geq 1$, es decir, $\mathbb{Z}_{p^{n}} \in \mathbb{F}_{\sigma}$ para toda $n \geq 1$. Por otro lado, si $\sigma \in\left[\alpha_{p}, 1_{\mathbb{Z}}\right]$, entonces $\alpha_{\mathbb{Z}_{p^{n}}}^{\mathbb{Z}_{p^{n}}} \preceq \sigma$ para toda $n \geq 1$ y por la Observación 1.57, $\sigma\left(\mathbb{Z}_{p^{n}}\right)=\mathbb{Z}_{p^{n}}$ para toda $n \geq 1$, de donde $\mathbb{Z}_{p^{n}} \in \mathbb{T}_{\sigma}$ para toda $n \geq 1$.

Observación 5.34. Notemos de la demostración del Corolario anterior que para $\sigma \in \mathbb{Z}$-radidem, los conjuntos $A_{\sigma}$ y $B_{\sigma}$ que se definieron en la Proposición 5.30 consisten de los primos que satisfacen 1 y 2 respectivamente del Corolario 5.33.

Si consideramos dos biparticiones distintas de $\mathbb{P}$, los intervalos que aparecen en el Corolario 5.31 son ajenos dos a dos, como veremos en la siguiente proposición. 
Proposición 5.35. Sean $(A, B) \neq\left(A^{\prime}, B^{\prime}\right)$, dos biparticiones de $\mathbb{P}$. Entonces $\left[\bigvee_{p \in A} \alpha_{p}, \bigwedge_{q \in B} \omega_{q}\right] \cap\left[\bigvee_{p \in A^{\prime}} \alpha_{p}, \bigwedge_{q \in B^{\prime}} \omega_{q}\right]=\emptyset$

Demostración. Supongamos que $\left[\bigvee_{p \in A} \alpha_{p}, \bigwedge_{q \in B} \omega_{q}\right] \cap\left[\bigvee_{p \in A^{\prime}} \alpha_{p}, \bigwedge_{q \in B^{\prime}} \omega_{q}\right] \neq$ $\emptyset$. Entonces $\bigvee_{p \in A^{\prime}} \alpha_{p} \preceq \bigwedge_{q \in B} \omega_{q}$. Es decir, para cada $p \in A^{\prime}$ y $q \in B$ tenemos que $\alpha_{p} \preceq \omega_{q}$, es decir, $\alpha_{\mathbb{Z}_{p^{n}}}^{\mathbb{Z}_{n}} \preceq \omega_{0}^{\mathbb{Z}_{q^{n}}}$ y por el Lema 5.26 obtenemos que $p \neq q$. De donde $A^{\prime} \cap B=\emptyset$ y por tanto $A^{\prime} \subseteq A$. Puesto que también $\bigvee_{p \in A} \alpha_{p} \preceq \bigwedge_{q \in B^{\prime}} \omega_{q}$, análogamente a lo anterior se prueba que $A \cap B^{\prime}=\emptyset$ y así $A \subseteq A^{\prime}$. Por lo tanto $A=A^{\prime}$ y de ahí que $B=B^{\prime}$. Entonces $(A, B)=\left(A^{\prime}, B^{\prime}\right)$.

Como consecuencia de la Proposición 5.35, para cada $\sigma \in \mathbb{Z}$-radidem existe una única bipartición $\left(A_{\sigma}, B_{\sigma}\right)$ asociada a él, la cual se define en la prueba de la Proposición 5.30, tal que $\sigma \in\left[\bigvee_{p \in A_{\sigma}} \alpha_{p}, \bigwedge_{q \in B_{\sigma}} \omega_{q}\right]$.

En seguida, mostraremos que cada uno de los intervalos cuya unión aparece en el Corolario 5.31, tiene al menos un elemento de $\mathbb{Z}$-radidem.

Proposición 5.36. Sea $(A, B) \in \mathcal{B}(\mathbb{P})$. Entonces

$$
\left[\bigvee_{p \in A} \alpha_{p}, \bigwedge_{q \in B} \omega_{q}\right] \cap \mathbb{Z} \text {-radid } \neq \emptyset
$$

Demostración. Puesto que $\bigwedge_{q \in B} \omega_{q}$ es un radical, tal que $\bigvee_{p \in A} \alpha_{p} \preceq \bigwedge_{q \in B} \omega_{q}$, entonces por la Proposición 1.74, $\bigvee_{p \in A} \alpha_{p} \preceq \overline{\bigvee_{p \in A} \alpha_{p}} \preceq \bigwedge_{q \in B} \omega_{q}$. Ahora, ya que $\bigvee_{p \in A} \alpha_{p}$ es un prerradical idempotente, por la Observación 1.75, $\overline{\bigvee \alpha_{p}}$ es un radical idempotente. Luego, por la Proposición 1.72, $\bigvee_{p \in A} \alpha_{p} \preceq \widehat{\bigwedge_{q \in B} \omega_{q}} \preceq \bigwedge_{q \in B} \omega_{q}$ y por la Observación 1.75, $\bigwedge_{q \in B} \omega_{q}$ también es un radical idempotente. Por lo tanto, concluimos que al menos existe un radical idempotente en tal intervalo.

De [7], se puede conluir que la clase de todos los radicales en $\mathbb{Z}$-pr no es un conjunto. También se sabe que la clase de todos los radicales exactos izquierdos 
sobre cualquier anillo es un conjunto. Pero aún no se sabe si $\mathbb{Z}$-radidem es un conjunto o no. Considerando que el número de biparticiones de $\mathbb{P}$ es $2^{\aleph_{0}}$, la unión de los intervalos que aparecen en el Corolario 5.31 es disjunta y por la Proposición anterior cada uno de tales intervalos tiene al menos un radical idempotente, podemos decir que si $\mathbb{Z}$-radid fuera un conjunto, entonces su cardinalidad sería al menos $2^{\aleph_{0}}$.

Por otro lado, ahora mostraremos que el extremo inferior de tales intervalos es un radical. Mas aún, es un radical exacto izquierdo y todos esos elementos son precisamente el conjunto de todos los radicales exactos izquierdos en $\mathbb{Z}$-pr. Para esto, primero probaremos el siguiente lema. Para cada $n \in \mathbb{Z}$, denotaremos por $\operatorname{pdiv}(n)$ al conjunto de todos los divisores primos de $n$. Para un elemento $x$ de orden finito en un $\mathbb{Z}$-módulo, denotaremos su orden como $o(x)$.

Lema 5.37. Sea $A \subseteq \mathbb{P}$ y sea $M \in \mathbb{Z}$-Mod. Entonces

$$
\left(\bigvee_{p \in A} \alpha_{p}\right)(M)=\{x \in M \mid o(x)<\infty, \operatorname{pdiv}(o(x)) \subseteq A\}
$$

Demostración. Sea $x \in\left(\bigvee_{p \in A} \alpha_{p}\right)(M)$. Por definición, $x=x_{1}+\cdots+$ $x_{m}$, donde $x_{i} \in \operatorname{Im}\left(f_{i}\right)$, con $f_{i}: \mathbb{Z}_{p_{i}^{n_{i}}} \rightarrow M$ y $p_{i} \in A$, para $i=1, \ldots, m$. Luego, $p_{i}^{n_{i}} x_{i}=0$ para $i=1, \ldots, m$, por lo que $\left(p_{1}^{n_{1}} p_{2}^{n_{2}} \cdots p_{m}^{n_{m}}\right) x=0$. Entonces $\operatorname{pdiv}(o(x)) \subseteq A$. Por otro lado, si $x \in M$ tal que $o(x)=p_{1}^{n_{1}} p_{2}^{n_{2}} \cdots p_{m}^{n_{m}}$ con $p_{i} \in A$ para $i=1, \cdots, m$, entonces existen $x_{1}, \ldots, x_{m} \in M$ con $o\left(x_{i}\right)=p_{i}^{n_{i}}$ para $i=1, \ldots, m$ tales que $\langle x\rangle=\left\langle x_{1}\right\rangle+\cdots+\left\langle x_{m}\right\rangle$. De donde concluimos, $x \in\left(\bigvee_{p \in A} \alpha_{p}\right)(M)$.

Proposición 5.38. Para cada $A \subseteq \mathbb{P}, \bigvee_{p \in A} \alpha_{p}$ es un radical.

Demostración. Sean $M \in \mathbb{Z}$-Mod y $\bar{x} \in\left(\bigvee_{p \in A} \alpha_{p}\right)\left(M /\left(\bigvee_{p \in A} \alpha_{p}\right)(M)\right)$. Entonces $\bar{x}=x+\left(\bigvee_{p \in A} \alpha_{p}\right)(M)$, donde $x \in M$. Existen $p_{1}, \ldots, p_{m} \in A \mathrm{y}$ $r_{1}, \ldots, r_{m} \geq 1$, tales que por el Lema 5.37, $\left(p_{1}^{r_{1}} \cdots p_{m}^{r_{m}}\right) x \in\left(\bigvee_{p \in A} \alpha_{p}\right)(M)$. De nuevo por el Lema 5.37, existen $q_{1}, \ldots, q_{n} \in A$ y $s_{1}, \ldots, s_{n} \geq 1$, tales que 
$\left(q_{1}^{s_{1}} \cdots q_{n}^{s_{n}}\right)\left(p_{1}^{r_{1}} \cdots p_{m}^{r_{m}}\right) x=0$. Por lo tanto $x \in\left(\bigvee_{p \in A} \alpha_{p}\right)(M)$, es decir, $\bar{x}=0$. Así de la Observación 1.66, se concluye que $\bigvee_{p \in A} \alpha_{p}$ es un radical.

Observe que en particular $\bigvee_{p \in \mathbb{P}} \alpha_{p}$ es la torsión usual, la cual es un radical idempotente, que asigna a cada grupo abeliano su subgrupo de torsión. Se sabe que hay una correspondencia biyectiva entre las topologías de Gabriel sobre un anillo $R$ y los radicales exactos izquierdos sobre $R$. De hecho, en [19], Stenström describe todas las topologías de Gabriel de un anillo noetheriano conmutativo, que es el caso del anillo $\mathbb{Z}$. Por la Proposición 5.38, dichas topologías de Gabriel estan en correspondencia biyectiva con los radicales exactos izquierdos $\bigvee_{p \in A} \alpha_{p}$, definidos para cada $A \subseteq \mathbb{P}$ y por tanto son todos los radicales exactos izquierdos de $\mathbb{Z}$-pr.

Para finalizar esta sección, mostraremos los intervalos en $\mathbb{Z}$-pr donde se encuentran ciertos radicales idempotentes y las biparticiones asociadas a ellos.

Ejemplo 5.39. Para el anillo $\mathbb{Z}$, el conjunto $\mathbb{Z}$-simp $=\left\{\mathbb{Z}_{p} \mid p \in \mathbb{P}\right\}$. Como en el Ejemplo 1.70, para cada $A \subseteq \mathbb{P}$, el Zoclo relativo se define como $\operatorname{Soc}_{A}\left(\_\right)=$ $\bigvee \alpha_{\mathbb{Z}_{p}}^{\mathbb{Z}_{p}}$. Por definición, Soc ${ }_{A}\left(\mathbb{Z}_{p}\right)=\mathbb{Z}_{p}$ para todo $p \in A$ y $\operatorname{Soc}_{A}\left(\mathbb{Z}_{q^{n}}\right)=0$ para $p \in A$

toda $n \geq 1$ y $q \in \mathbb{P} \backslash A$. Entonces $\mathbb{Z}_{p} \in \mathbb{T}_{\text {Soc }_{A}\left(\_\right)} \subseteq \mathbb{T}_{\overline{\text { Soc }_{A}(} \text { _) }}$ para todo $p \in A$, También, para todo $q \in \mathbb{P} \backslash$ A y $n \geq 1$, se tiene $\mathbb{Z}_{q^{n}} \in \mathbb{F}_{\text {Soc }_{A}\left(\_\right)}=\mathbb{F}_{\overline{\text { Soc }_{A}}\left(\_\right)}$, donde la última igualdad es por la Proposición 1.76. Luego, por la Observación 5.34, $\mathbb{Z}_{p^{n}} \in \mathbb{T} \overline{\operatorname{Soc}}_{A}\left({ }_{\text {_ }}\right.$ para todo $p \in A$ y toda $n \geq 1$ y por la misma Observación $\overline{\operatorname{Soc}_{A}}\left(\_\right) \in\left[\alpha_{p}, 1_{\mathbb{Z}}\right]$, para toda $p \in A$. Similarmente, $\overline{\operatorname{Soc}_{A}}\left(\_\right) \in\left[0, \omega_{q}\right]$ para toda $q \in \mathbb{P} \backslash A$. Por lo tanto la bipartición correspondiente al radical idempotente $\overline{\operatorname{Soc}_{A}}\left(\_\right)$es $(A, \mathbb{P} \backslash A)$ y asi $\overline{\operatorname{Soc}_{A}}\left(\_\right) \in\left[\bigvee_{p \in A} \alpha_{p}, \bigwedge_{q \in \mathbb{P} \backslash A} \omega_{q}\right]$.

Por otro lado, para cada $p \in A, \alpha_{\mathbb{Z}_{p}}^{\mathbb{Z}_{p}} \preceq \alpha_{p}$, por lo que $\operatorname{Soc}_{A}\left(\_\right) \preceq \bigvee_{p \in A} \alpha_{p}$. Por la Proposición 5.38, $\bigvee_{p \in A} \alpha_{p}$ es un radical, entonces por la Proposición 1.74, $\overline{\operatorname{Soc}_{A}}\left(\_\right) \preceq \bigvee_{p \in A} \alpha_{p}$. De lo anterior, $\overline{\operatorname{Soc}_{A}}\left(\_\right)=\bigvee_{p \in A} \alpha_{p}$. En particular, $\overline{\operatorname{Soc}}\left(\_\right)=$ $\bigvee_{p \in \mathbb{P}} \alpha_{p}$ y su correspondiente bipartición es $(\mathbb{P}, \emptyset)$.

Ejemplo 5.40. Dualmente, del Ejemplo 1.70, para cada $B \subseteq \mathbb{P}$, el radical de Jacobson relativo se define como $\operatorname{Rad}_{B}\left(\_\right)=\bigwedge_{q \in B} \omega_{0}^{\mathbb{Z}_{q}}$. Por definición, $\operatorname{Rad}_{B}\left(\mathbb{Z}_{q}\right)=$ 
0 para todo $q \in B$ y $\operatorname{Rad}_{B}\left(\mathbb{Z}_{p^{n}}\right)=\mathbb{Z}_{p^{n}}$ para toda $n \geq 1$ y $p \in \mathbb{P} \backslash$ B. Entonces, para todo $p \in \mathbb{P} \backslash$ B y $n \geq 1$ se tiene que $\mathbb{Z}_{p^{n}} \in \mathbb{T}_{\text {Rad }_{B}\left({ }_{-}\right)}=\mathbb{T}_{\left.\widehat{\operatorname{Rad}_{B}(}\right)} y$ la última igualdad es por la Proposición 1.76. También, para todo $q \in B, \mathbb{Z}_{q} \in$ $\mathbb{F}_{\text {Rad }_{B}\left(\_\right)} \subseteq \mathbb{F}_{\widehat{\operatorname{Rad}_{B}}\left(\_\right)}$. Análogamente al ejemplo 5.39, por la Observación 5.34, la bipartición correspondiente al radical idempotente $\widehat{\operatorname{Rad}_{B}}\left(\_\right)$es $(\mathbb{P} \backslash B, B) y$ $\widehat{\operatorname{Rad}_{B}}\left(\_\right) \in\left[\bigvee_{p \in \mathbb{P} \backslash B} \alpha_{p}, \bigwedge_{q \in B} \omega_{q}\right]$. De lo anterior, $\widehat{\operatorname{Rad}_{B}}\left(\_\right) \preceq \widehat{\bigwedge_{q \in B} \omega_{q}}$.

Por otro lado, para cada $q \in B, \omega_{q} \preceq \omega_{0}^{\mathbb{Z}_{q}}$, por lo que $\bigwedge_{q \in B} \omega_{q} \preceq \operatorname{Rad}_{B}\left(\_\right) y$ asi $\bigwedge_{q \in B} \omega_{q} \preceq \widehat{\operatorname{Rad}}\left(\_\right)$. Por lo tanto $\widehat{\operatorname{Rad}_{B}}\left({ }_{(}\right)=\widehat{\bigwedge_{q \in B} \omega_{q}}$. En particular, $\widehat{\operatorname{Rad}}\left({ }_{-}\right)=$ $\bigwedge_{q \in \mathbb{P}} \omega_{q}$ y su correspondiente bipartición es $(\emptyset, \mathbb{P})$. Es decir, $\widehat{\operatorname{Rad}}\left(\_\right) \in\left[0_{\mathbb{Z}}, \bigwedge_{p \in \mathbb{P}} \omega_{p}\right]$. Nótece que para cada $M \in \mathbb{Z}-M o d,\left(\bigwedge_{p \in \mathbb{P}} \omega_{p}\right)(M)$ es el primer subgrupo de Ulm de $M$ y $\widehat{\operatorname{Rad}}(M)$ es el mayor submódulo divisible de $M$ (Para mayor información ver [12]).

\subsection{Particiones de $\mathbb{Z}_{p^{n}}$-pr inducidas por ideales}

Como mencionamos al inicio de este capítulo aprovecharemos que la retícu-

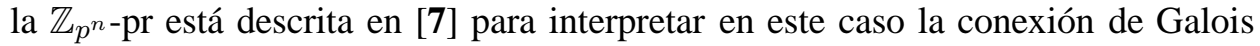
$\left\langle\varphi_{I}, \psi_{I}\right\rangle$, donde $I=p^{i} \mathbb{Z}_{p^{n}}$ es un ideal de $\mathbb{Z}_{p^{n}}$.

Consideremos un ideal $I=p^{i} \mathbb{Z}_{p^{n}}$ de $\mathbb{Z}_{p^{n}}$, donde $p$ primo, $n \geq 2$ e $i=$ $1, \ldots, n-1$. Por la Proposición 4.3, la conexión de Galois $\left\langle\varphi_{I}, \psi_{I}\right\rangle$, queda descrita como:

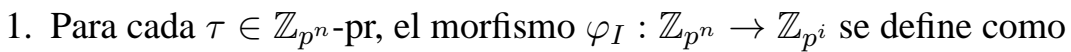

$$
\varphi_{I}(\tau)=\sigma
$$

donde $\sigma$ es la restricción de $\tau$ a los $\mathbb{Z}_{p^{i}}$-módulos. Así, si $\tau$ tiene la siguiente representación binaria $\tau=\left(a_{1}, \ldots, a_{i}, \ldots, a_{n}\right)$, entonces $\sigma=$ $\left(a_{1}, \ldots, a_{i}\right)$.

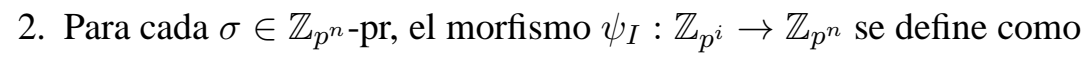

$$
\psi_{I}(\sigma)=\tau
$$

donde $\tau$ es la mayor extensión de $\sigma$ en los $R$-módulos. Así, si $\sigma$ tiene la siguiente representación binaria $\sigma=\left(a_{1}, \ldots, a_{i}\right)$, entonces $\tau=$ $\left(a_{1}, \ldots, a_{i}, 1,1, \ldots, 1_{n}\right)$. 
En esta conexión de Galois la descripción de los intervalos en $\mathbb{Z}_{p^{n} \text {-pr inducidos }}$ por la imagen inversa de elementos de $\mathbb{Z}_{p^{i}}$-pr bajo $\varphi_{I}$ quedan descritos de una manera muy sencilla, como veremos enseguida.

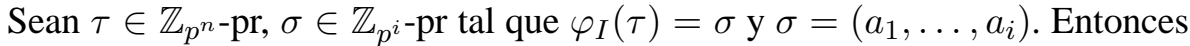
por la Observación 4.20:

$$
\overleftarrow{\varphi_{I}}(\sigma)=\left[\left(a_{1}, \ldots, a_{i}, 0 \ldots, 0_{n}\right),\left(a_{1}, \ldots, a_{i}, 1 \ldots, 1_{n}\right)\right]
$$

Puesto que los intervalos en (88) inducen una partición de $\mathbb{Z}_{p^{n}}$-pr se tiene que la cardinalidad de cada intervalo es $2^{m-i}$.

Para fijar estas ideas veamos el siguiente ejemplo.

Ejemplo 5.41. Consideremos el anillo $R=\mathbb{Z}_{p^{3}}$ y el ideal $I=p^{2} \mathbb{Z}_{p^{3}}$. Entonces la conexión de Galois inducida por el ideal I es $\left\langle\varphi_{I}, \psi_{I}\right\rangle$, donde $\varphi_{I}: \mathbb{Z}_{p^{3}}-p r \rightarrow$ $\mathbb{Z}_{p^{2}-p r y} \psi_{I}: \mathbb{Z}_{p^{2}-p r} \rightarrow \mathbb{Z}_{p^{3}}-p r$.

Por (86) se tiene que $\varphi_{I}$ evaluado en un $\mathbb{Z}_{p^{3}}$-prerradical $\tau$, resulta la restricción de $\tau$ en los $\mathbb{Z}_{p^{2}}$-módulos. Así, el morfismo $\varphi_{I}$ en todos los $\mathbb{Z}_{p^{3}}$-prerradicales es:

- $\varphi_{F}(0,0,0)=\varphi_{F}(0,0,1)=(0,0)$

- $\varphi_{F}(0,1,0)=\varphi_{F}(0,1,1)=(0,1)$

- $\varphi_{F}(1,0,0)=\varphi_{F}(1,0,1)=(1,0)$

- $\varphi_{F}(1,1,0)=\varphi_{F}(1,1,1)=(1,1)$

De ahí es claro lo siguiente:

- $\overleftarrow{\varphi_{I}}(0,0)=[(0,0,0),(0,0,1)]=\left[(0,0,0), \alpha_{I}^{R}\right]$

- $\overleftarrow{\varphi_{I}}(0,1)=[(0,1,0),(0,1,1)]$

- $\overleftarrow{\varphi_{I}}(1,0)=[(1,0,0),(1,0,1)]$

- $\overleftarrow{\varphi}_{I}(1,1)=[(1,1,0),(1,1,1)]=\left[t\left(\alpha_{I}^{R}\right),(1,1,1)\right]$

donde la última igualdad de los renglones 1 y 4 se siguen de los Corolarios 4.17 y 4.18. Notemos que lo anterior también se puede concluir de (88).

Luego por (87) las imágenes bajo $\psi_{I}$ de todos los $\mathbb{Z}_{p^{2}}$-prerradicales son:

- $\psi_{I}(0,0)=(0,0,1)$

- $\psi_{I}(0,1)=(0,1,1)$

- $\psi_{I}(1,0)=(1,0,1)$

- $\psi_{I}(1,1)=(1,1,1)$.

Lo anterior también se puede concluir de la descripción de los intervalos inducidos por la imagen inversa bajo $\varphi_{I}$ que aparece en la Proposición 4.15. Note que en efecto, la descripción de $\psi_{I}$ corresponde a la mayor extensión de los $\mathbb{Z}_{p^{2}}$ prerradicales a los $\mathbb{Z}_{p^{3}}$-módulos.

También, por la Proposición 4.19, sabemos que los únicos elementos cerrados en $\mathbb{Z}_{p^{3}-p r \text { son: }}$

$$
(0,0,1),(0,1,1),(1,0,1),(1,1,1) .
$$

Por último, puesto que $\psi_{I}$ es inyectiva todos los elementos de $\mathbb{Z}_{p^{2}}$-pr son abiertos. Recordemos de la Proposición 1.20 del Capítulo 1, que hay un isomorfismo entre 


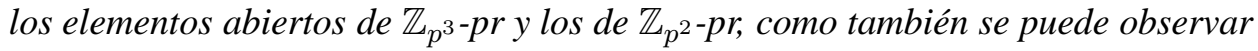
en este ejemplo.

\subsection{Particiones de $\mathbb{Z}_{(p)}$-pr inducidas por ideales}

En esta sección estudiaremos otro ejemplo particular de conexion de Galois entre las retículas $R$-pr y $R / I$-pr, considerando el anillo local $\mathbb{Z}_{(p)}$ el cual es la localización del anillo $\mathbb{Z}$ en el primo $p$. Tal anillo tiene como único ideal máximo a $p \mathbb{Z}_{(p)}$ y su retícula de ideales es una cadena infinita como sigue:

$$
\cdots<I_{i}<\cdots<I_{2}<I_{1}<\mathbb{Z}_{(p)}
$$

donde $I_{i}=p^{i} \mathbb{Z}_{(p)}$ para $i \geq 1$. Ya que $\mathbb{Z}_{(p)} / p^{i} \mathbb{Z}_{(p)} \cong \mathbb{Z} / p^{i} \mathbb{Z}$ para $i \geq 1$, de nuevo

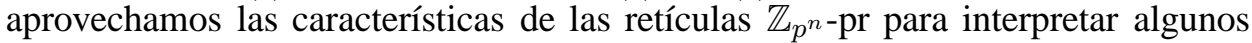
resultados de las secciones anteriores a la conexión de Galois $\left\langle\varphi_{I}, \psi_{I}\right\rangle$ inducida

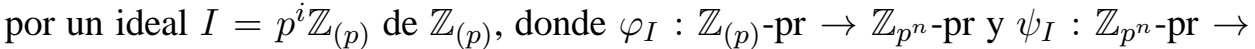
$\mathbb{Z}_{(p)}$-pr.

Por ser un caso particular de conexión de Galois entre retículas $R$-pr y $R / I$ pr, los morfismos $\varphi_{I}$ y $\psi_{I}$ quedan definidos como en la Proposición 4.3 y por la estructura del anillo $\mathbb{Z}_{(p)}$ los resultados más interesantes para esta conexión de Galois son con respecto a los intervalos en $\mathbb{Z}_{(p)}$-pr en donde se encuentran los $\mathbb{Z}_{(p)}$-prerradicales idempotentes, radicales y radicales idempotentes. Para esto, observamos que la igualdad (64) sigue teniendo validez en este caso, puesto que dicha igualdad se sigue de la Proposición 5.3 en donde se usa que el anillo $\mathbb{Z}_{p^{n}}$ es local uniserial. Así en este caso, los prerradicales idempotentes en $\mathbb{Z}_{(p)}$-pr de acuerdo a la expresión (77) se encuentran en:

$$
\mathbb{Z}_{(p)} \text {-idem } \subset\left[0, \bigwedge_{n=1}^{\infty} \omega_{0}^{\mathbb{Z}_{p^{n}}}\right] \cup \bigcup_{m=1}^{\infty}\left[\alpha_{\mathbb{Z}_{p}^{m}}^{\mathbb{Z}_{p^{m}}}, \bigwedge_{n=m+1}^{\infty} \omega_{\mathbb{Z}_{p^{m}}}^{\mathbb{Z}_{p^{n}}}\right] \cup\left[\bigvee_{n=1}^{\infty} \alpha_{\mathbb{Z}_{p^{n}}}^{\mathbb{Z}_{p^{n}}}, 1\right]
$$

De acuerdo a la expresión (81), los radicales en $\mathbb{Z}_{(p)}$-pr se encuentran en:

$$
\mathbb{Z}_{(p)}-\operatorname{rad} \subset\left[0, \bigwedge_{n=1}^{\infty} \omega_{0}^{\mathbb{Z}_{p^{n}}}\right] \cup \bigcup_{k=1}^{\infty}\left[\bigvee_{n=k+1}^{\infty} \alpha_{\mathbb{Z}_{p^{n-k}}}^{\mathbb{Z}_{p^{n}}}, \omega_{0}^{\mathbb{Z}_{p^{k}}}\right] \cup\left[\bigvee_{n=1}^{\infty} \alpha_{\mathbb{Z}_{p^{n}}}^{\mathbb{Z}_{p^{n}}}, 1\right] .
$$

Y por último la expresión (85) muestra en este caso que los intervalos en $\mathbb{Z}_{(p)^{-}}$ pr en donde podemos encontrar a los $\mathbb{Z}_{(p)}$ radicales idempotentes solamente son dos:

$$
\mathbb{Z}_{(p)} \text {-radidem } \subset\left(\left[0, \omega_{p}\right] \bigcup\left[\alpha_{p}, 1\right]\right)
$$





\section{Conclusiones y Perspectivas}

Dedicamos este último espacio para formular algunos problemas siguiendo en la línea de este trabajo. El objetivo principal de esta investigación fué estudiar la relación entre dos retículas de prerradicales $R$-pr y $S$-pr a partir de alguna relación entre los anillos $R$ y $S$. El Teorema 2.46, el más importante de este trabajo, da lugar al estudio de las retículas de prerradicales correspondientes a las categorías de módulos cuando hay una situación de adjunción entre ellas. En este caso hemos mostrado que hay una conexión de Galois entre las retículas relacionadas. Una aplicación de dicho resultado fue el estudio de la conexión de Galois $\left\langle R\right.$-pr, $\varphi_{I}, \psi_{I}, R / I$-pr $\rangle$, que resultó muy fructífera puesto que el morfismo $\varphi_{I}$ evaluado en $\tau \in R$-pr es la restricción de $\tau$ a los $R / I$-módulos y en el caso de $\psi_{I}$ evaluado en un $\sigma \in R / I$-pr es la mayor extensión de $\sigma$ a los $R$-módulos, de donde obtenemos varios resultados, que después se aplican a otras conexiones de Galois más específicas dentro de este caso. Aprovechando que en [7] se caracteriza

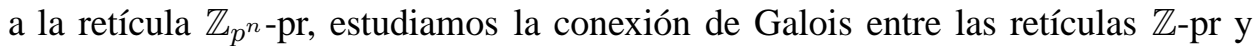

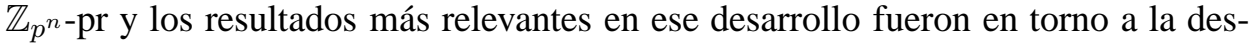
cripción de las particiones en $\mathbb{Z}$-pr inducidas por la imagen inversa bajo $\varphi_{I}$ de un

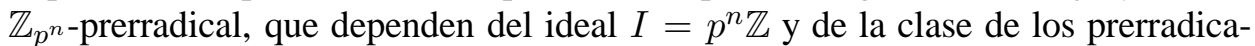
les idempotentes y radicales sobre $\mathbb{Z}_{p^{n}}$. Determinamos también las particiones de otras retículas de prerradicales asociadas a grupos abelianos e ideales $p^{n} \mathbb{Z}$, teniendo como referencia el caso de $\mathbb{Z}$-pr.

Como podemos observar tenemos varios caminos en los cuales podemos dirigirnos siguiendo este tipo de estudio. A continuación listamos algunas posibles líneas de investigación.

1. De acuerdo al Teorema 2.46, podemos explorar otros pares adjuntos y la conexión de Galois inducida por ellos. Por ejemplo los funtores de Coxeter o de reflexión entre las categorías de módulos de álgebras de caminos que se definen a partir del cambio en la orientación de la flechas correspondientes a una fuente o a un sumidero. Por ejemplo ver el Capítulo VII.5 de [15].

2. El Teorema de Watts-Eilenberg caracteriza a los funtores adjuntos entre categorías de módulos y en el segundo capítulo estudiamos la conexión de Galois inducida por los funtores $F=U \bigotimes_{R}$ y y $G=\operatorname{Hom}_{S}\left(U,{ }_{-}\right)$, donde $U={ }_{S} U_{R}$ es un bimódulo, que caracterizan a los pares adjuntos entre las categorías de módulos. La Proposición 2.67 muestra cómo son las evaluaciones de los morfismos $\varphi$ y $\psi$ que forman parte de la conexión de Galois inducida por tales funtores, en los prerradicales correspondientes. Esta descripción como $\tau$-traza y $\sigma$-anulador es muy 
interesante porque debido al Teorema de Watts-Eilenberg todos esos morfismos evaluados tienen esa forma y podemos continuar su estudio. Una buena referencia en esta dirección son los ejercicios de la Sección 19 de [2], con respecto al $\sigma$-anulador donde $\sigma \in S$-pr.

3. Además de estudiar conexiones de Galois entre retículas de prerradicales inducidas por una situación de adjunción entre las categorías de módulos, en el tercer capítulo abordamos una situación más general, y por tanto más usual, entre retículas de prerradicales. Esto es, definiendo morfismos entre las retículas de prerradicales a partir de transformaciones naturales, lo cual da lugar para analizar diversas situaciones. Por ejemplo, estudiamos el caso en donde la transformación natural es la identidad sobre un funtor asociando de esta manera un morfismo entre retículas de prerradicales a cualquier funtor entre categorías de módulos. En particular cuando el funtor es un prerradical, obtuvimos de una manera alternativa los prerradicales igualador, coigualador y anulador que aparecen en [9]. Se destaca el totalizador como imagen del prerradical identidad bajo el morfismo $\varphi$ asociado al funtor inclusión (o funtor que olvida) $u_{\sigma}$ con $\sigma \in R$-pr, que es de naturaleza distinta a los otros tres casos, por lo cual no se obtuvieron los mismos resultados.

Aún podemos plantearnos varias preguntas con respecto a los morfismos entre retículas de prerradicales asociados a funtores. Una pregunta relevante es si se vale el recíproco del Corolario 3.23. Es decir, si $F, F^{\prime}: R$-Mod $\rightarrow S$-Mod son funtores tales que $\varphi_{F}=\varphi_{F^{\prime}}$ y $\psi_{F}=\psi_{F^{\prime}}$, ¿será que $F \cong F^{\prime}$ ?. En otras palabras, si podemos caracterizar salvo isomorfismo funtores entre categorías de módulos a partir de los dos tipos de morfismos entre retículas de prerradicales asociados.

4. El caso de la conexión de Galois entre las retículas $R$-pr y $R / I$-pr donde $I$ es un ideal de $R$ que se aborda en el Capítulo cuatro, nos sugiere que podemos investigar otras conexiones de Galois de esa forma, es decir, podríamos trabajar con anillos de tal forma que la retícula de prerradicales asociada al anillo cociente sea relativamente fácil de trabajar y a partir de las propiedades en el cociente obtener información acerca de una retícula de prerradicales más complicada, tomando como referencia lo que se desarrolló en el Capítulo 5 , en el caso de $\mathbb{Z}$-pr.

5. Además de estudiar situaciones en donde tengamos un homomorfismo suprayectivo $f: R \rightarrow S$ de anillos, se puede considerar una situación más general, considerando un epimorfismo de anillos en el sentido categórico. Como es sabido hay epimorfismos de anillos que no son suprayectivos, pero aún en este caso también se tiene un funtor $G: S$-Mod $\rightarrow R$-Mod que induce un morfismo entre las retículas de prerradicales respectivas. En particular, podemos estudiar un epimorfismo plano entre anillos, es decir, cuando $S$ es un $R$-módulo plano, situación donde al anillo $S$ se le llama la localización perfecta del anillo $R$. Ver el Capítulo XI de [19]. 


\section{Bibliografía}

[1] Adámek, J., Herrlich, H., Strecker, G., Abstract and concrete categories: the joy of cats. John Wiley and Sons, 2004.

[2] Anderson, F.W., Fuller, K. R., Rings and categories of modules. Springer Verlag, New York, 1974.

[3] Bican, L., Jambor, P., Kepka, T., Nẽmec, P., Preradicals and change of rings. Comment. Math. Univ. Carolinae. 16 (2), 201- 217, 1975.

[4] Bican, L., Kepka, T., Nẽmec, P., Rings, modules and preradicals. Marcel Dekker, INC, New York and Basel, 1982.

[5] Eilenberg., S., Abstract description of some basic functors. J. Indian Math. 24, 231-234, 1960.

[6] Erné, M., Koslowski, J., Melton, A., Strecker, G., A primer on Galois connections. Annals of the New York Academy of Sciencies. 704, 103-125, 1993.

[7] Férnandez-Alonso, R., Gavito, S., The lattice of Prerradicals over Local Uniserial Rings. Journal of Algebra and its Aplications. 5 (6), 731-746, 2006.

[8] Férnandez-Alonso, R., Raggi, F., Rincón, H., Ríos, J., Signoret, C., The lattice structure of Prerradicals. Commun. Algebar. 30 (3), 1533-1544, 2002.

[9] Fernández-Alonso, R., Raggi, F., Ríos, J., Rincón, H., Signoret, C., The lattice structure of preradicals II. Partitions. Journal of Algebra and its Applications. 1(2), 201-214, 2002.

[10] Férnandez-Alonso, R., Raggi, F., Rincón, H., Ríos, J., Basic Preradicals and Main Injective Modules. Commun. Algebar. 30 (3), 1533-1544, 2002.

[11] Férnandez-Alonso, R., Gavito, S., Chimal-Dzul, H., A class of rings for which the lattice of Preradicals is not a set. International Electronic Journal of Algebra. 9 , 38-60, 2011. 
[12] Fuchs L., Infinite Abelian Groups, Vol. I. Academic Press, New York, 1970.

[13] Grätzer, G., General lattice Theory. Birkhäauser Verlag, Winnipeg, Manitoba, 2003.

[14] Herrlich, H., Strecker, G., Category theory: an introduction. Allyn \& Bacon, 1973.

[15] Ibrahim, A., Simson, D., Skowroński, A., Elements of the representation theory of associative algebras. Volume 1 techniques of representation theory. Cambridge, University, Press, New York, 2006.

[16] Mac Lane, S., Categories for the working mathematician. Springer Verlag, New York, 1998.

[17] Roman, S., Lattices and Ordered Sets. Springer Verlag, 2008.

[18] Shmuely, Z., The structure of Galois connections. Pacific Journal of Mathematics. 54 (2), 209-225, 1974.

[19] Stenström, B., Rings of quotients an introduction to methods of rings theory. Springer Verlag, New York, 1975.

[20] Watts, C. E., Intrinsic characterization of some additive functors. Proc. Amer. Math. Soc. 11, 5-8, 1960. 


\section{Índice alfabético}

Anillos Morita-equivalentes, 17, 37

Anulador de un prerradical, 27, 95

Átomos

en $R$-pr, 23

en $\mathbb{Z}$-pr, 121

Bipartición, 130

Categorías equivalentes, 17, 31

Clase

$\operatorname{Pr}(\mathcal{A}), 29$

$\operatorname{Pr}^{*}(\mathcal{A}), 40$

Coátomos

en $R$-pr, 23

en $\mathbb{Z}$-pr, 121

Coigualador de un prerradical, 27, 93

Composición

de transformaciones naturales, 16

Conexión de Galois, 13, 43, 98

Conjunto parcialmente ordenado (copo), 11

Coprerradical, 42, 70

Coproducto de prerradicales, 24, 102

Elemento

abierto, 13, 105

cerrado, 13, 105

máximo, 11

mínimo, 11

mayor, 11

menor, 11

Epimorfismo, 18, 19

Función

deflatoria, 12, 46

idempotente, 12

inflatoria, 12, 45

Funtor

adjunto izquierdo, 18

denso, 17

fiel, 17

Hom contravariante, 16
Hom covariante, 15, 55

pleno, 17

producto tensorial, 55

Igualador de un prerradical, 27, 92

Ínfimo, 11, 21

Isomorfismo

de copos, 12

de retículas, 12

natural, 16

Monomorfismo, 18, 19

Morfismo

de copos, 12

de retículas, 12

Operador

cerradura, 12, 102, 109

interior, 12, 102, 109

Orden parcial, 11

Prerradical, 20

$\alpha_{h}, 47$

alfa, 22, 112

exacto izquierdo, 27, 133

idempotente, 24, 53

idempotente en $\mathbb{Z}$-pr, 124

idempotente en $\mathbb{Z}_{p^{n} \text {-pr }, 112}$

$\omega_{k}, 47$

omega, 22, 112

$\bar{\sigma}, 26$

$\widehat{\sigma}, 26$

$t$-radical, 27

Producto

de elementos en $\operatorname{Pr}(\mathcal{A}), 37$

de elementos en $\operatorname{Pr}^{*}(\mathcal{A}), 41$

de prerradicales, 23, 102

estrella de transformaciones naturales, 16

Radical (prerradical), 24, 53, 103

en $\mathbb{Z}$-pr, 126 
en $\mathbb{Z}_{p^{n} \text {-pr, } 112}$

Radical de Jacobson, 20

en $\mathbb{Z}$-pr, 120

relativo, 25

relativo en $\mathbb{Z}$-pr, 135

Rechazo, 20

Retícula, 12

$\mathbb{Z}_{p^{n} \text {-pr, } 111}$

completa, 12

$\sigma$-anulador respecto a $U, 56$

Sistema

de abiertos, 13

de cerrados, 13

Situación de adjunción entre categorías, 18

Submódulo totalmente invariante, 22, 23

Supremo, 12, 21

$\tau$-traza respecto a $U, 55$

Totalizador de un prerradical, 27

Transformación natural, 15

Traza, 20

Zoclo, 20

en $\mathbb{Z}$-pr, 119

relativo, 25

relativo en $\mathbb{Z}$-pr, 134 